\title{
Jacobienne locale d'une courbe formelle relative
}

\author{
Carlos Contou-Carrère
}

ABstRACT - This article is devoted to the proof of a relative duality formula on a noetherian scheme $S$, giving rise on the spectrum of a field $S=$ Spec $k$ to local symbols of class field theory. Relative local symbols are obtained in terms of the universal property of a couple ( $\Im, f$ ), of a $S$-group functor $\Im$, associated to a $S$ formal curve $\mathfrak{X}$ locally of the form $\mathfrak{X}=\operatorname{Spf} A[[T]](S=\operatorname{Spec} A)$. $\Im$ is a $S$-group extension of the completion $\breve{W}$ of the universal $S$-Witt vectors group $W$, by the group of units $\mathcal{O}_{S}[[T]]^{*}$. We associate an $S$-functor $\Im_{\text {omb }}$ to $\Im$, and we define an Abel-Jacobi morphism $f: \mathfrak{U}=\operatorname{Spec} A[[T]]\left[T^{-1}\right] \longrightarrow \Im_{\text {omb }}$, setting up a group isomorphism:

$$
\operatorname{Hom}_{S-g r}(\Im, G) \simeq G(\mathfrak{l}),
$$

where $G$ denotes a commutative smooth $S$-group scheme. We define an $S$-bihomomorphism

$$
\Im \times \Im \longrightarrow \mathrm{G}_{m},
$$

which is a local symbol (The Tame Symbol), identifying $\Im$ to its own Cartier dual group $\breve{\Im}=\underline{\operatorname{Hom}}_{S-g r}\left(\Im, \mathrm{G}_{m}\right)$, and inducing the above isomorphism for $G=\mathrm{G}_{m}$. It follows that $\Im$ may be interpreted as the relative Loop Group:

$$
\mathrm{G}_{m}(\mathfrak{U}): S^{\prime} \longrightarrow \mathrm{G}_{m}\left(\mathfrak{U}_{\left\{S^{\prime}\right\}}\right),
$$

$S^{\prime}=\operatorname{Spec} A^{\prime}$ denotes a $S$-scheme, and we write $\mathfrak{U}_{\left\{S^{\prime}\right\}}=\operatorname{Spec} A^{\prime}[[T]]\left[T^{-1}\right]$, and as the $A$-universal group of Witt-Bivectors.

The couple $(\Im, f)$ may be seen as the local analogue of the relative Rosenlicht Jacobian (Generalized Jacobian) defined by a $S$-smooth curve $X$.

Mathematics Subject Classification (2010). 14L15.

KeYwords. Local Symbol(s), Tame Symbol, Contou-Carrere Symbol, Local AbelJacobi morphism, Universal Witt Bivectors, Cartier Duality, Local Jacobian, Relative Formal Curve, Witt Residues, Local Relative Class Field Theory, Rosenlicht Jacobian.

(*) Indirizzo dell'A.: Université Montpellier II Laboratoire Charles Coulomb UMR 5221 F-34095 Montpellier France.

E-mail: ccontouc@univ-montp2.fr 


\section{Introduction}

Le but de ce travail est d'interpréter le symbole local du corps de classes géométrique (cf. [14]) en termes d'une proprieté universelle d'un couple $(\mathfrak{J}, f)$, ou $\mathfrak{J}$ est un foncteur en groupes au-dessus d'un schéma noethérien $S$, associe à une courbe formelle $\mathfrak{X}$ localement de la forme $\mathfrak{X}=\operatorname{Spf} A[[T]]$, ou $S=\operatorname{Spec} A$, et où $f$ désigne un morphisme de type Abel-Jacobi de $\mathfrak{U}=\operatorname{Spec} A[[T]]\left[T^{-1}\right]$ vers un $S$-foncteur $\mathfrak{J}_{\text {omb }}$ défini par $\mathfrak{I}$, et induisant une bijection fonctorielle

$$
\operatorname{Hom}_{S-g r}(\mathfrak{J}, G) \simeq G(\mathfrak{H}),
$$

pour un $S$-schéma en groupes commutatif lisse et séparé $G$ (cf. (**) plus bas).

Le cas $G=\mathrm{G}_{m}$ de cette bijection est particulièrement intéressant et peut-être explicité comme l'autodualité de Cartier de $\mathfrak{J}$. La formule qui rend cette auto-dualité est désignée par le symbole moderé.

Le symbole moderé intervient depuis plus d'une dizaine d'années dans plusieurs travaux concernant en particulier le corps de classes local et les rapports entre ce dernier et la physique théorique, pour lesquels on confère le lecteur aux reférences supplémentaires. Une version analytique de ce dernier est donné par P. Deligne dans [D]. G. Anderson et P. Romos ont demontré une formule de reciprocité pour celui-ci (cf. [A-R]), permettant de retrouver plusieurs lois de reciprocité pour une courbe complète audessus d'un corps algébriquement clos. P. Romos a démontré la proprieté de Steinberg (cf. [PR]). M. Kapranov, et E. Vasserot formulent et prouvent un théorème local de Riemann-Roch pour des gerbes determinantielles, où la auto-dualité de $\mathfrak{J}$ joue un rôle central (cf. [K-V]). Le symbole moderé intervient dans la definition des algèbres chirales (cf. [Bei-Dr]), ainsi que dans les calculs des $\varepsilon$-facteurs pour les determinants de Gauss-Manin (cf. [Bei], [B-B-E]).

Le présent article s'insère dans un programme de travail sur les dualités globales et locales à coefficients continus proposés par A. Grothendieck à l'auteur (confer aussi la lettre de A. Grothendieck à J. P. Serre du 10/8/60). Ce programme comporte dans un premier temps l'extension de la formule de dualité de [7], pour une $S$-courbe propre et lisse, à coefficients dans un $S$-schéma en groupes $G$ commutatif et plat, au cas d'une $S$-courbe lisse. Dans [3] et [5] on construit une jacobienne généralisée relative pour une courbe lisse $X-D$, donnée par le complémentaire d'un diviseur relatif fermé $D$ d'une courbe propre $X$ sur une base $S$, qui vérifie certaines hypothèses, et un morphisme d'Abel-Jacobi $\Phi: X-D \longrightarrow J$ qui permet 
d'établir un isomorphisme de groupes

$$
\operatorname{Hom}_{S-g r}(J, G) \simeq G(X-D),
$$

pour tout $S$-schéma en groupes lisse $G$. (Cette construction constitue l'extension naturelle des jacobiennes de Rosenlicht-Serre au cas relatif, et un premier pas vers la démonstration d'une formule de dualité en termes de champs de Picard associés à $X-D$ ). L'étude des jacobiennes généralisés globales mène à l'introduction des symboles locaux à valeurs dans un $S$-schéma en groupes $G$. Ces symboles locaux ont été introduits par J. P. Serre dans [14] dans le cas où $S$ est le spectre d'un corps.

La jacobienne généralisée $J$ est une extension de la jacobienne Pic $_{X / S}$ de la courbe propre $X$ par le $S$-groupe $\mathfrak{J}^{0}$ de nature locale autour de $D$, défini par

$$
\mathfrak{J}^{0}: S^{\prime} \longrightarrow \Gamma\left(D^{\prime}, \mathcal{O}_{\widehat{X}_{/ D^{\prime}}^{*}}\right),
$$

où $S^{\prime}$ est un $S$-schéma, $\widehat{X}_{/ D^{\prime}}$ désigne le schéma formel obtenu par changement de base à partir du complété formel $\mathfrak{X}=\widehat{X}_{/ D}$ de $X$ le long $\operatorname{de} D$, et $\mathcal{O}_{\widehat{X}_{/ D}^{*}}^{*}$ le faisceau de groupes des unités de $\mathcal{O}_{\widehat{X}_{/ D^{\prime}}}$.

Supposons $S=\operatorname{Spec}(A)$ affine et posons $\operatorname{omb}\left(\widehat{X}_{/ D}\right)=$ $\operatorname{Spec}\left(\Gamma\left(\widehat{X}_{/ D}, \mathcal{O}_{\widehat{X}_{/ D}}\right)\right)$. Par la bijection (*), la donnée d'une section $s$ de $G$ au-dessus de $X-D$ donne lieu à un $S$-homomorphisme $\psi: J \longrightarrow G$ dont la restriction $\psi^{\prime}$ à $\mathfrak{J}^{0} \subset J$ (symbole local donné par $\psi$ ) dépend essentiellement de la restriction de $s$ à $\mathfrak{U}=\operatorname{omb}\left(\widehat{X}_{/ D}\right)-D$, c.à.d. de la partie singulière de $s$ le long de $D$. Un des résultats de ce travail (cf. Théorème (1.4.4)) est de montrer que à toute courbe formelle lisse de la forme $\mathfrak{X} \simeq \operatorname{Spf}\left(\mathcal{O}_{S}[[T]]\right)=\operatorname{Spf}(A[[T]])$, au dessus d'une base affine et noethérienne $S$, on peut associer un $S$-groupe $\mathfrak{J}^{0}$ et un morphisme

$$
\varphi^{0}: \mathfrak{U}=\operatorname{Spec}\left(\Gamma\left(\mathfrak{X}, \mathcal{O}_{\mathfrak{X}}\right)\right)-D \longrightarrow \mathfrak{J}^{0}(D=V(T))
$$

vérifiant la propriété universelle suivante : pour tout $S$-schéma en groupes $G$ lisse et séparé on a une bijection

$$
\operatorname{Hom}_{S-g r}\left(\mathfrak{J}^{0}, G\right) \simeq G(\mathfrak{U}) / G(\operatorname{omb}(\mathfrak{X})),
$$

où $\operatorname{omb}(\mathfrak{X})=\operatorname{Spec}\left(\Gamma\left(\mathfrak{X}, \mathcal{O}_{\mathfrak{X}}\right)\right)=\operatorname{Spec}(A[[T]])$. En plus si $\mathfrak{X}=\widehat{X}_{/ D}$, on a alors que $\Phi$ et $\varphi^{0}$ coïncident sur $J(\mathfrak{l})$ à une section de $J(\operatorname{omb}(\mathfrak{X}))$ près.

L'énoncé de (1.4.4) a été proposé à titre conjecturel par A. Grothendieck.

Pour se débarrasser de l'hypothèse $A$ noethérien il suffit de montrer 
l'énoncé suivant qui permet de ramener la preuve de (1.4.4) au cas noethérien. Etant donné $\bar{\xi} \in G(\mathfrak{H}) / G(\mathrm{omb}(\mathfrak{X}))$ il existe une Z-sous-algèbre $A^{\prime} \subset A$ de type fini et $\xi \in G\left(\operatorname{Spec}\left(A^{\prime}[[T]]\left[T^{-1}\right]\right)\right)$ tel que sa restriction à

$$
G(\mathfrak{H})=G\left(\operatorname{Spec}\left(A[[T]]\left[T^{-1}\right]\right)\right)
$$

induit $\bar{\xi}$ par passage au quotient.

Notons par $L_{D} \subset \mathcal{O}_{\mathfrak{X}}$ l'idéal de définition du diviseur $V(T)=D \subset \mathfrak{X}$. On désigne par $\mathcal{O}_{\mathfrak{X}}\left[L_{D}^{-1}\right]$ le faisceau en anneaux de fractions de $\mathcal{O}_{\mathfrak{X}}$ par rapport aux sections de l'idéal $L_{D}$ qui sont localement des générateurs, et par $\mathcal{O}_{\mathfrak{x}}\left[L_{D}^{-1}\right]^{*}$ le faisceau des unités de $\mathcal{O}_{\mathfrak{x}}\left[L_{D}^{-1}\right]$. Soit $S^{\prime}$ un $S$-schéma. On pose

$$
\mathfrak{X}^{\prime}=\mathfrak{X} \times_{S} S^{\prime} \text { et } D^{\prime}=D \times_{S} S^{\prime},
$$

et on désigne par $L_{D^{\prime}}$, l'idéal de définition de $D^{\prime}$ dans $\mathfrak{X}^{\prime}$. On définit le faisceau $\mathcal{O}_{\mathfrak{X}}\left[L_{D^{\prime}}^{-1}\right]^{*}$ comme ci-dessus à partir $\mathfrak{X}^{\prime}, D^{\prime}$, et $L_{D^{\prime}}$, à la place de $\mathfrak{X}, D$, et $L_{D}$. On définit un $S$-faisceau de Zariski en groupes commutatif par

$$
\Im: S^{\prime} \longrightarrow \Gamma\left(D^{\prime}, \mathcal{O}_{\mathfrak{x}}\left[L_{D^{\prime}}^{-1}\right]^{*}\right) .
$$

Ce $S$-groupe est le sujet principal de ce travail. On donne une interprétation des sections de $\Im$ en termes d'idéaux fractionnaires inversibles de $\mathcal{O}_{\mathfrak{X}}$ munis d'une trivialisation, ce qui dans le cas où $\mathfrak{X}=\widehat{X}_{/ D}$ entraîne l'existence d'un morphisme naturel de $S$-groupes $\Im \longrightarrow J$ reliant $\Im$ et la jacobienne généralisée. Ce $S$-groupe $\Im$ apparaît naturellement même dans le cas d'un corps de base $k$. En supposant $k$ algébriquement clos, pour fixer les idées, on a que le groupe des idèles est un sous-groupe du produit des groupes $\Im$ correspondant aux diviseurs $x$ définis par les points de $X$ à valeurs dans $k: \prod_{x} \Im_{x}$.

Le $S$-groupe $\Im$ est une extension du complété formel du groupe des vecteurs de Witt universel $\check{W}$, introduit dans [1] par Cartier, par un $S$ groupe (pro-lisse) $\mathfrak{J}$ extension de $\underline{Z}_{S}$ par $\mathfrak{J}^{0}$. Le théorème principal (cf. Théorème (1.6.6)) de ce travail montre que $\Im$ a la propriété universelle suivante : pour tout $S$-schéma en groupes commutatif lisse et séparé $G$ on a un isomorphisme de groupes:

$$
\operatorname{Hom}_{S-g r}(\Im, G) \simeq G(\mathfrak{H}),
$$

qui résulte de la propriété universelle de $\breve{W}$ vis à vis des morphismes de $\mathfrak{X}$ dans un groupe formel, (cf. [2]), et de celle du couple $\left(\mathfrak{J}^{0}, \varphi^{0}\right)$ (cf. Théorème (1.4.4)), et peut s'interpréter comme une formule de dualité locale.

Le $S$-groupe $\Im$ est muni d'un auto-accouplement, anti-symétrique que l'on explicite dans $\S 6$,

$$
\langle\ldots, \ldots\rangle: \Im \times \Im \longrightarrow \underline{\mathrm{G}} m S,
$$


et qui fait que $\Im$ soit isomorphe à son propre dual de Cartier, c.à.d.

$$
\underline{\operatorname{Hom}}_{S-g r}\left(\Im, \underline{\mathrm{G}}_{m S}\right) \stackrel{\sim}{\longrightarrow} \Im .
$$

Noter d'autre part que $\Gamma(\mathfrak{U}, \underline{\mathbb{G}} m S)=\Gamma(S, \Im)$. La flèche induite par $\langle\ldots, \ldots\rangle$ coïncide alors avec celle donnée par la propriété universelle de $\Im$ si $G=\underline{G} m s$. On a un isomorphisme

$$
\Im \simeq \check{W} \times \underline{\mathbb{Z}}_{S} \times \underline{\mathrm{G}} m S \times \wedge^{0} .
$$

Le $\langle\ldots, \ldots\rangle$ induit l'accouplement entre $\check{W}$ et $\wedge^{0}=\operatorname{Ker}\left(\mathfrak{J}^{0} \longrightarrow\right.$ G $\left.m s\right)$, ou $\mathfrak{J}^{0} \longrightarrow \underline{\mathrm{G}} m S$ désigne le morphisme naturel, donné dans [1].

La construction de l'isomorphisme $(* *)$ passe par celle d'un morphisme d'Abel-Jacobi, version locale du morphisme $\Phi$. Remarquons d'abord que même dans le cas d'un corps de base $S=\operatorname{Spec}(k)$ il n'existe pas de morphisme fonctoriel $\mathfrak{U} \longrightarrow \Im$ induisant l'isomorphisme $(* *)$. En effet comme $\mathfrak{U} \simeq \operatorname{Spec}\left(k[[T]]\left[T^{-1}\right]\right)$ alors $W(\mathfrak{H})$ se réduit à la section donnée par l'élément neutre de $\check{W}$. D'où si un tel morphisme existait on aurait

$$
\operatorname{Hom}_{S-g r}(\check{W}, G) \subseteq \operatorname{Ker}\left(\operatorname{Hom}_{S-g r}(\Im, G) \longrightarrow G(\mathfrak{H})\right),
$$

où la flèche est induite par composition avec $\mathfrak{U} \longrightarrow \Im$.

On construit alors un objet intermédiaire $\Im_{\text {omb }}$, qui est un $S$-foncteur, entre les $S$-schémas formels et les $S$-schémas, et un morphisme

$$
f: \mathfrak{U} \longrightarrow \Im_{\text {omb }},
$$

tel que l'on a une inclusion $\Im \subset \Im_{\text {omb }}$, et à tout $S$-homomorphisme $h: \Im \longrightarrow G$ dans un $S$-schéma en groupes $G$ commutatif et lisse, il correspond un unique morphisme $h_{\mathrm{omb}}: \Im_{\mathrm{omb}} \longrightarrow G$ dont la restriction à $\Im$ coïncide avec $h$. On démontre finalement que $f$ induit l'isomorphisme (**). Notons que $\Im_{\text {omb }}$ n'est pas un $S$-foncteur en groupes.

Pour poser le problème universel dont $\Im$ est solution dans le cas où l'on se donne un schéma noethérien $S$ de base, et une $S$-courbe formelle $\mathfrak{X}$, isomorphe à $\operatorname{Spf}\left(\mathcal{O}_{S}[[T]]\right)$, autant que pour donner une interprétation géométrico-fonctorielle de $\Im_{\text {omb }}$, il est nécessaire de revenir sur la construction de l'ombre d'un schéma formel noethérien, suggérée par A. Grothendieck, qui n’a jamais été traitée systématiquement.

On donne dans $\S 0$ le matériel minimum indispensable à nos besoins. (A la conaissance de l'auteur il n'existe pas de références). Les constructions de (1.5) sont ménées en supposant $\mathfrak{X}$ isomorphe à $\operatorname{Spf}\left(\mathcal{O}_{S}[[T]]\right)$ et $S$ affine et noethérien. Il est alors immédiat que l'on peut dans le cas général donner un sens à l'énoncé du théorème principal, qui résulte alors d'une 
conséquence du cas traité par nous, et étendre la construction de $\Im_{\text {omb...etc, }}$ a ce cas. On peut interpréter omb( $\mathfrak{X})$ comme un "trait relatif" au-dessus de $S, \mathfrak{U}$ comme son point générique, et le $S$-groupe $\Im=\underline{\mathbb{G}} \mathrm{mS}(\mathfrak{l})$ comme "l'espace des lacets relatifs de G $\mathrm{ms}$ ".

Le $S$-groupe $\breve{W}$ n'étant pas un $S$-schéma formel noethérien, la première difficulté est de définir omb $(\breve{W})$. On définit omb $(\check{W})$ comme une limite inductive de la catégorie des $S$-schémas $\left.S \operatorname{sh}\right|_{S}$ si $S$ est affine et noethérien, et comme une limite inductive de la catégorie des $S$ espaces localement annelés Locann $\left.\right|_{S}$ si $S$ est un schéma noethérien. Alors $\Im_{\text {omb }}$ apparaît comme une limite inductive de $\left.S \operatorname{sch}\right|_{S}$ (resp. Locann $\left.\right|_{L}$ ) si $S$ est affine et noethérien (resp. noethérien) muni d'une structure de $\mathfrak{J}$-torseur au-dessus de omb $(\breve{W})$. On donne ensuite une description géométrique du $S$-foncteur $\Im_{\text {omb }}$, qui en entraîne une autre du morphisme d'Abel-Jacobi $f: \mathfrak{U} \longrightarrow \Im_{\text {omb }}$, et permet d'établir la compatibilité de $f$ avec $\Phi$.

Disons un mot sur la structure de la démonstration du théorème (1.6.6). Elle est ramenée à celle de (1.4.4). On traite d'abord le cas d'un corps de base. On retrouve alors essentiellement les symboles locaux correspondant au groupe multiplicatif $\underline{\mathrm{G}} \mathrm{mS}$ et aux tronqués des groupes additifs des vecteurs de Witt (symbole de Witt). On montre ensuite comment le théorème (1.4.4), dans le cas d'un anneau noethérien réduit de base, entraîne le même pour tout anneau noethérien de base. On introduit à cette fin une catégorie de $S$-faisceaux fppf en groupes commutatifs, définie à partir des propriétés (A) et (B) (cf. § 3), pour lesquels le théorème (1.4.4) est valable, et ayant certaines propriétés de permanence. Soit $S_{0}=\operatorname{Spec}(A / I)$ avec $I^{2}=0$. Si $G$ est un $S$-groupe comme dans l'hypothèse du théorème (1.4.4) soit $G_{0}=G \times_{S} S_{0}$ alors l'image directe $G^{+}=i_{*} G_{0}$ par le morphisme naturel $i:\left(S_{0}\right)_{\text {fppf }} \longrightarrow S_{\text {fppf }}$ appartient à cette catégorie. Finalement en supposant vrai le théorème (1.4.4) pour tout anneau réduit de base et pour $S_{0}$ on prouve, à partir de la suite exacte $1 \longrightarrow K \longrightarrow G \longrightarrow G^{+} \longrightarrow 1$ (cf. (3.3)), que $G$ vérifie les conditions (A) et (B) donc la conclusion du théorème (1.4.4). En combinant ce résultat avec le précédent on obtient (1.4.4) pour le spectre d'un anneau artinien de base. Dans $\S 5$ on voit comment la preuve de (1.4.4) pour une base noethérienne réduite se ramène au cas d'un trait complet de base $S$, c.à.d. le spectre $S=\operatorname{Spec}(V)$ d'un anneau de valuation discrète complet $V$. La démonstration de (1.4.4) pour $S=\operatorname{Spec}(V)$ constitue la clé de voute de la preuve de (1.4.4). Au début du $§ 4$ (après (4.1.5) bis) on explique la stratégie de la preuve de (1.4.4) dans ce cas. Elle est basée sur le fait suivant. Soit $\theta_{n}: \mathfrak{U} \longrightarrow \mathfrak{U}$ le $S$-morphisme fini et fidèlement plat défini par le $V$-homomorphisme $V[[T]]\left[T^{-1}\right] \longrightarrow V[[T]]\left[T^{-1}\right]$ donné par $T \mapsto T^{n}$. On a alors que pour toute section $\alpha \in G(\mathfrak{U})$ il existe un 
entier $n$ tel que si $n^{\prime}, n^{\prime \prime}>n$ sont des entiers de même parité on a $(* * *)$

$$
\operatorname{tr}_{\theta_{n^{\prime}}} \alpha \equiv \operatorname{tr}_{\theta_{n^{\prime \prime}}} \alpha(\bmod G(\operatorname{omb}(\mathfrak{X})))
$$

(Il s'agit là d'un avatar local du résultat suivant concernant les jacobiennes généralisées au-dessus d'un corps $k$ qui résulte de la Proposition 9, pg. 47 de [14] et des constructions suivantes. Soient $X, D$ comme ci-dessus. Soit $h \in G(X-D)$. Il existe un entier $n$ vérifiant les trois propriétés équivalentes suivantes :

- Pour tout morphisme fini et plat $g: X \longrightarrow \mathbb{P}_{k}^{1}$ tel que $g^{-1}(0)=D^{(n)}(n$ voisinage infinitésimal de $D$ dans $X$ ) on a que $\operatorname{tr}_{g} h: \mathbb{P}_{k}^{1}-\{0\} \longrightarrow G$ admet un prolongement à $\mathbb{P}_{k}^{1}$ et il est alors constant.

- Le morphisme $h$ se factorise par $X-D \longrightarrow J \longrightarrow \underline{\operatorname{Pic}}_{X^{(n)} / k}$, où $X^{(n)}$ désigne la somme amalgammée $X \prod_{D^{(n)}} \operatorname{Spec}(k)$.

- La section $h$ induit un morphisme constant sur chaque $D^{(n)}$-classe d'équivalence linéaire (cf. loc. cit.).) Il est une conséquence immédiate de (1.4.4) pour un corps. On passe au cas général grâce à l'adaptation schématique (cf. 4.1.15) d'un lemme de pureté de Weil (cf. [18]), pour les morphismes rationnels d'une variété dans un groupe algébrique $G$. Le théorème (1.4.4) pour le spectre d'un anneau artinien de base nous permet de construire une factorisation formelle $\widehat{\alpha}=f \circ \widehat{\varphi}^{0}$ (cf. (4.1.4)) de $\alpha$, où $f: \widehat{\mathfrak{J}}^{0} \longrightarrow G$ désigne un $S$-homomorphisme du complété formel le long de la fibre spéciale $\widehat{\mathfrak{J}}^{0}$ de $\widehat{\mathfrak{J}}^{0}$ dans $G$, et $\widehat{\alpha}: \widehat{\mathfrak{U}} \longrightarrow G\left(\right.$ resp. $\left.\widehat{\varphi}^{0}: \widehat{\mathfrak{U}} \longrightarrow \widehat{\mathfrak{J}}^{0}\right)$ le morphisme induit par $\alpha$ (resp. $\varphi^{0}$ ) par restriction au complété formel $\widehat{\mathfrak{U}}$ de $\mathfrak{U}$ le long de la fibre spéciale. Grâce à $(* * *)$ et à une application du lemme d'algébrisation (cf. (4.2.1)) d'un morphisme de la "fibre géométrique omb $(\widehat{U})_{K}$ " du complété formel $\widehat{U}$ le long de la fibre spéciale de l'ouvert $U \subset \mathbb{P}_{S}^{1}$, complémentaire du diviseur 0 et du diviseur $\infty$ de $\mathbb{P}_{S}^{1}$, dans un espace projectif $\mathbb{P}_{S}^{N}$, vérifiant certaines conditions locales d'algébricité on conclut que $f$ provient d'un morphisme algébrique.

Soit $S^{\prime} \longrightarrow S$ une extension fidèlement plate affine du schéma noethérien affine $S$. Plusieurs fois dans ce travail on utilise le fait que la bijection

$$
\operatorname{Hom}_{S^{\prime}-g r}\left(\mathfrak{I}_{S^{\prime}}^{0}, G_{S^{\prime}}\right) \stackrel{\sim}{\longrightarrow} G\left(\mathfrak{U}_{\left\{S^{\prime}\right\}}\right) / G\left(\operatorname{omb}\left(\mathfrak{X}_{S^{\prime}}\right)\right)(\mathrm{cf} .(0.4))
$$

induite par $\varphi_{S^{\prime}}^{0}: \mathfrak{U}_{S^{\prime}} \longrightarrow \mathfrak{J}_{S^{\prime}}^{0}$, entraîne le théorème (1.4.4). Ce résultat est un corollaire de l'injectivité de la flèche

$$
\operatorname{Hom}_{S-g r}\left(\mathfrak{J}^{0}, G\right) \longrightarrow G(\mathfrak{U}) / G(\operatorname{omb}(\mathfrak{X}))
$$


pour toute base affine $S$ (cf. Proposition (1.4.5)) dont la preuve dépend des propriétés de la jacobienne généralisée de la droite projective $\mathrm{P}_{S}^{1}$ moins le diviseur de l'infini et de l'origine, que l'on calcule explicitement dans (1.3).

Le processus de démonstration du (1.4.4) est constructif (notamment les dévissage concernant les anneaux artiniens qui semblent mener à des symboles de type Kawada-Satake (cf. [18]), ainsi que les constructions pour un anneau de valuation discrète $V$ ).

L’isomorphisme $(* *)$ entraîne une suite exacte scindée

$$
1 \longrightarrow G(\operatorname{omb}(\mathfrak{X}))^{+} \longrightarrow G(\mathfrak{U}) \longrightarrow G(\mathfrak{U}) / G(\operatorname{omb}(\mathfrak{X}))^{+} \longrightarrow 1,
$$

où $G(\operatorname{omb}(\mathfrak{X}))^{+}=\operatorname{Ker}(G(\operatorname{omb}(\mathfrak{X})) \longrightarrow G(S))$. Noter que $G(\operatorname{omb}(\mathfrak{X}))$ ne dépend que du complété formel $\widehat{G}$ le long de la section unité. Autant la structure de $G(\mathfrak{X})$ comme module au-dessus de l'anneau des endomorphismes de $\wedge$ permet de récupérer $\widehat{G}$, autant l'action des opérateurs $V_{n}$ et $F_{n}$ sur $G(\mathfrak{U}) / G(\mathrm{omb}(\mathfrak{X}))$ (cf. [2]) doit permettre de donner une décomposition des symboles locaux et une description de la structure de $G(\mathfrak{U})$ (d'interpréter dans ce contexte les courbes typiques...etc).

Les remarques suivantes indiquent dans quel sens l'étude de ce travail peut être poursuivie. La filtration naturelle de $\mathfrak{J}^{0}$ dont les quotients sont les $\mathfrak{J}_{n}^{0}$ doit aussi se retrouver dans $G(\mathfrak{U}) / G(\operatorname{omb}(\mathfrak{X}))$ grâce à $(* *)$ et au fait essentiel que $\operatorname{Hom}_{S-g r}\left(\mathfrak{J}^{0}, G\right)=\lim \operatorname{Hom}_{S-g r}\left(\mathcal{J}_{n}^{0}, G\right)$ lorsque $G$ est localement de présentation finie. La considération de la structure de cette filtration semble livrer des renseignements intéressants sur $G$.

D'autre part, vu les arguments de $\S 3$, il est naturel de se demander pour quels $S$-faisceaux en groupes $G$ les théorèmes (1.4.4) et (1.6.6) sont vrais, ensuite d'étudier pour ces groupes les propriétés de la flèche naturelle

$$
\begin{gathered}
\operatorname{Ext}_{S-g r}^{1}\left(\mathfrak{J}^{0}, G\right) \longrightarrow H^{1}(\mathfrak{H}, G) \\
\left(\operatorname{resp} . \operatorname{Ext}^{1}\left(\mathfrak{J}^{0}, G\right) \times H^{1}(\operatorname{omb}(\mathfrak{X}), G) \longrightarrow H^{1}(\mathfrak{H}, G)\right)
\end{gathered}
$$

déduite de $\varphi_{T}^{0}$. Autrement dit, il s'agit d'étudier les liens entre les catégories de Picard $\operatorname{EXT}\left(\mathfrak{J}^{0}, G\right)$, TORS $(\mathfrak{U}, G)$, et TORS(omb( $\left.\left.\mathfrak{X}\right), G\right)$ pour arriver plus généralement à comparer les objets $R \operatorname{Hom}\left(\mathfrak{J}^{0}, G\right)$ et $R \Gamma(\mathfrak{U}, G)$.

Il semble que le théorème (1.6.6) joint aux résultats de J. P. Serre (cf. Sur les corps locaux à corps résiduel algébriquement clos, B.S.M.F., vol. 89, 1961) permettent de prouver, pour les courbes lisses au-dessus d'un corps une formule de dualité qui correspond à celle de [7].

Resterait à établir les rapports entre ce travail et ceux de I. Barsotti sur les propriétés des groupes des bi-vecteurs de Witt en connexion avec les 
variétés abéliennes en caractéristique positive (compte tenu des rapports entre les extensions de la jacobienne $J$ d'une courbe propre et lisse $X$ par un groupe affine commutatif avec les jacobiennes généralisés, et de ces dernières avec $\mathfrak{I})$.

La possibilité de l'existence de l'extension $\mathfrak{I}$ fut conjecturée par A. Grothendieck.

\section{Définitions préliminaires}

Soit $A$ un anneau unitaire commutatif. On désigne par $A[[T]]$ la $A$-algèbre adique des séries formelles à coefficients dans $A$. Soit $B$ une $A$-algèbre adique augmentée isomorphe à $A[[T]]$. On note $I$ l'idéal de $B$ donné par le noyau du morphisme canonique $B \longrightarrow A$. Étant donnée une $A$-algèbre $A^{\prime}$ on désigne par $B \widehat{\otimes}_{A} A^{\prime}$ le produit tensoriel complété pour la topologie $I$-adique. On pose

$$
B_{\left\{A^{\prime}\right\}}=B \widehat{\otimes}_{A} A^{\prime}
$$

et

$$
I_{\left\{A^{\prime}\right\}}=I B_{\left\{A^{\prime}\right\}} .
$$

Noter que pour $B=A[[T]]$, on a $B_{\left\{A^{\prime}\right\}} \simeq A^{\prime}[[T]]$ canoniquement.

Il est clair que $B_{\{A\}}=B$ et $I_{\{A\}}=I$. Un générateur $T$ de $I$ donne lieu à un générateur de $I_{\left\{A^{\prime}\right\}}$, que l'on note toujours $T$. L'anneau des fractions $B_{\left\{A^{\prime}\right\}}\left[T^{-1}\right]$ est indépendant du choix de $T$ et on le note $B_{\left\{A^{\prime}\right\}}\left[I_{\left\{A^{\prime}\right\}}^{-1}\right]$. Soit $n$ un entier naturel. On pose $B_{n}={ }^{B} / I^{n+1}$. On a alors $B_{n} \otimes_{A} A^{\prime}=B_{\left\{A^{\prime}\right\}} / I_{\left\{A^{\prime}\right\}}^{n+1}$. Soit

$$
\mathfrak{X}=\operatorname{Spf}(B) \quad\left(\operatorname{resp} . \mathfrak{X}_{n}=\operatorname{Spec}\left(B_{n}\right)\right)
$$

Soit $S=\operatorname{Spec}(A)$. Étant donné un $S$-schéma $S^{\prime}$, on désigne par $\mathfrak{X}^{\prime}=\mathfrak{X}_{S^{\prime}}=\mathfrak{X} \times{ }_{S} S^{\prime}$ le $S^{\prime}$-schéma formel obtenu à partir du $S$-schéma formel $\mathfrak{X}$ par le changement de base $S \longrightarrow S^{\prime}$. Soit $A^{\prime}$ une $A$-algèbre. Posons $S^{\prime}=\operatorname{Spec}\left(A^{\prime}\right)$. On a alors

$$
\mathfrak{X}_{S^{\prime}}=\operatorname{Spf}\left(B_{\left\{A^{\prime}\right\}}\right)
$$

que l'on note aussi $\mathfrak{x}_{A^{\prime}}$. On pose

$$
\operatorname{omb}\left(\mathfrak{X}_{S^{\prime}}\right)=\operatorname{omb}\left(\mathfrak{X}_{A^{\prime}}\right)=\operatorname{Spec}\left(B_{\left\{A^{\prime}\right\}}\right)
$$

et

$$
\mathfrak{U}=\operatorname{Spec}\left(B\left[I^{-1}\right]\right) \quad\left(\operatorname{resp} . \mathfrak{U}_{\left\{S^{\prime}\right\}}=\mathfrak{U}_{\left\{A^{\prime}\right\}}=\operatorname{Spec}\left(B_{\left\{A^{\prime}\right\}}\left[I_{\left\{A^{\prime}\right\}}^{-1}\right]\right)\right)
$$


Remarquer que $\operatorname{omb}\left(\mathfrak{x}_{S^{\prime}}\right)$ (resp. $\left.\mathfrak{U}_{\left\{S^{\prime}\right\}}\right)$ n'a de sens à priori que pour $S^{\prime}$ affine, et que l'objet $\mathfrak{U}_{\left\{S^{\prime}\right\}}$ dépend de $\mathfrak{X}$ et pas seulement du $S$-schéma $\mathfrak{U}$.

Soit $S^{\prime}$ un $S$-schéma. Soit $\mathcal{O}_{\mathfrak{X}^{\prime}}\left[T^{-1}\right]$ le faisceau d'anneaux de fractions de $\mathcal{O}_{\mathfrak{x}^{\prime}}$ à dénominateurs dans le sous-faisceau d'ensembles $\mathcal{S}$, engendré par la section $T$ de $\mathcal{O}_{\mathfrak{X}^{\prime}}$ donnée par un générateur $T$ de $I$ (cf. [11], (20.1.1), pg. 226). Le faisceau $\mathcal{O}_{\mathfrak{x}^{\prime}}\left[T^{-1}\right]$ est indépendant du choix de $T$.

On désigne par $\mathcal{O}_{\mathfrak{X}^{\prime}}^{*}$ (resp. $\left.\mathcal{O}_{\mathfrak{X}^{\prime}}\left[T^{-1}\right]^{*}\right)$ le faisceau des unités de $\mathcal{O}_{\mathfrak{X}^{\prime}}$ (resp. $\left.\mathcal{O}_{\mathfrak{X}^{\prime}}\left[T^{-1}\right]\right)$. Soit $\underline{G}_{m S}(\mathfrak{X})\left(\right.$ resp. $\left.\underline{G}_{m S}(\mathfrak{U})\right)$ le $S$-foncteur en groupes défini par

$$
\underline{\mathrm{G}}_{\mathrm{mS}}(\mathfrak{X})\left(S^{\prime}\right)=\Gamma\left(\mathfrak{X}^{\prime}, \mathcal{O}_{\mathfrak{X}^{\prime}}^{*}\right) \quad\left(\operatorname{resp} \cdot \underline{\mathrm{G}}_{\mathrm{mS}}(\mathfrak{U})\left(S^{\prime}\right)=\Gamma\left(\mathfrak{X}^{\prime}, O_{\mathfrak{X}^{\prime}}\left[T^{-1}\right]^{*}\right)\right) .
$$

Il résulte de la définition que $\underline{G}_{m S}(\mathfrak{X})\left(\right.$ resp. $\left.\underline{G}_{m S}(\mathfrak{l})\right)$ est un $S$-faisceau de Zariski sur le grand site. On pose

$$
\Im=\underline{\mathrm{G}}_{\mathrm{mS}}(\mathfrak{U}) \quad\left(\operatorname{resp} \cdot \mathfrak{J}^{0}=\underline{\mathrm{G}}_{\mathrm{mS}}(\mathfrak{X})\right) .
$$

Soit $A^{\prime}$ une $A$-algèbre. Posons $S^{\prime}=\operatorname{Spec}\left(A^{\prime}\right)$. On a alors

$$
\underline{\mathrm{G}}_{\mathrm{mS}}(\mathfrak{X})\left(S^{\prime}\right)=B_{\left\{A^{\prime}\right\}}^{*}\left(\operatorname{resp} . \underline{\mathrm{G}}_{\mathrm{mS}}(\mathfrak{l})\left(S^{\prime}\right)=B_{\left\{A^{\prime}\right\}}\left[I_{\left\{A^{\prime}\right\}}^{-1}\right]^{*}\right) \text {. }
$$

Done si $B=A[[T]]$ on a $\underline{G}_{\mathrm{mS}}(\mathfrak{X})\left(S^{\prime}\right)=A^{\prime}[[T]]^{*} \quad\left(\right.$ resp. $\underline{G}_{\mathrm{mS}}(\mathfrak{l} \mathfrak{l})\left(S^{\prime}\right)=$ $\left.A^{\prime}[[T]]\left[T^{-1}\right]^{*}\right)$.

Les deux lemmes suivants nous permettent de décrire $\Im$ en termes de $S$-groupes connus.

Lemme 0.7. Toute unité u de $A[[T]]\left[T^{-1}\right]$, c'est à dire $u \in A[[T]]\left[T^{-1}\right]^{*}$, s'écrit d'une façon unique de la forme:

$$
u=T^{-}\left(a_{-r} T^{-r}+\ldots+a_{-1} T^{-1}+a_{0}+\sum_{i \geq 1} a_{i} T^{i}\right),
$$

avec $\underline{n} \in \Gamma(\operatorname{Spec}(A), \underline{Z})$ avec $a_{-r}, \ldots, a_{-1}$ des nilpotents de $A$, et $a_{0}$ un élément inversible de $A$, c.à.d. $a_{0} \in A^{*}$.

Preuve. Écrivons $u=\sum_{i \in Z} b_{i} T^{i}$, et posons pour tout $p \in \operatorname{Spec}(A)$ :

$$
\mu(p)=\inf \left\{i \mid b_{i} \text { non-nilpotent dans } A_{p}\right\},
$$

et

$$
v(p)=\inf \left\{i \mid b_{i} \notin p\right\} .
$$

Voyons que $\mu=v$, et que $v$ est une fonction localement constante sur $\operatorname{Spec}(A)$ (et définit ainsi une section de $\underline{Z}$ ). 
Montrons que $\mu(p)=\inf v(p)(q \subseteq p)$ (le inf pris sur l'ensemble des

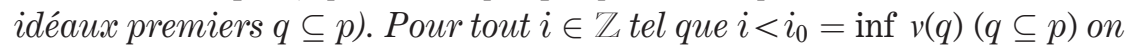
$a b_{i} \in \bigcap q A_{p}=$ Nilradical de $A_{p}$ (Donc $b_{i}$ est nilpotent). Comme il existe $q$ tel que $i_{0}=v(q)$ on a $b_{i_{0}} \notin q$ d'où $b_{i_{0}}$ est non-nilpotent dans $A_{p}$. Ce qui prouve bien l'égalité.

Noter d'autre part que $q \subseteq p \Rightarrow v(q) \leq v(p)$. Or l'unité u donne lieu à une unité dans $(A / q A)[[T]]\left[T^{-1}\right]$. Comme l'anneau $A / q A$ est intègre on en déduit que la classe $\bar{b}_{v(q)}$ de $b_{v(q)}$ dans $A / q A$ est inversible. La considération de l'homomorphisme $A / q A \longrightarrow A / p A$ entraîne que la classe $\bar{b}_{v(q)}$ de $b_{v(q)}$ dans $A / p A$ est un élément inversible de $A / p A$ donc que $b_{v(q)} \notin p$. D'où que $v(q)=v(p)$. Si A est un anneau local d'idéal maximal $m$ les deux égalités précédentes donnent $\mu(m)=v(m)$, ce qui entraîne l'énoncé de la proposition dans le cas où $A$ est local.

Soit $p \in \operatorname{Spec} A$. Si on applique la proposition à l'image $u_{p}$ de $u$ dans $A_{p}[[T]]\left[T^{-1}\right]$ on obtient que $b_{v(p)}$ est un élément inversible dans $A_{p}$, et que $b_{i}$ est nilpotent dans $A_{p}$ si $i<v(p)$. Il existe alors $f \in A$ tel que :

$f \notin p, a_{-r}, \ldots, a_{-1}$ sont des nilpotents dans $A_{f}$, et $a_{0} \in A_{f}^{*}$. On en déduit la proposition dans le cas général.

Lemme 0.8. Soit $C$ une sous-A-algèbre de $A[[T]]$ contenant $T$ et tel que $A \simeq C / T C$. Alors toute unité u de $C\left[T^{-1}\right]$ s'écrit de façon unique sous la forme $u=T^{n} u^{\prime} u^{\prime \prime}$ oì $\underline{n} \in \Gamma\left(\operatorname{Spec} A, Z^{\prime}\right), u^{\prime} \in C^{*}$, et $u^{\prime \prime}=a_{-r} T^{-r}+\ldots+a_{-1} T^{-1}+1$ avec $a_{-r}, \ldots, a_{-1}$ nilpotents dans $A$.

Preuve. Cas où $C=A[[T]]$. Quitte à localiser $A$, on peut supposer que:

$$
u=\left(a_{-r} T^{-r}+\ldots+a_{-1} T^{-1}+a_{0}+\sum_{i \geq 1} a_{i} T^{i}\right)
$$

avec $a_{-r}, \ldots, a_{-1}$ des nilpotents de $A$ et $a_{0}$ une unité de $A$.

Soit $v \in A[[T]]\left[T^{-1}\right]^{*}$ de la forme : $v=\alpha_{-p} T^{-p}+\ldots+\alpha_{-1} T^{-1}+\alpha_{0}+$ $\sum_{i>1} \alpha_{i} T^{i}$ avec $\alpha_{-p} \ldots \alpha_{-1}$ des nilpotents, et $\alpha_{0}$ une unité. On écrit alors

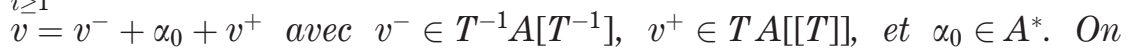
définit par récurrence une suite d'éléments $u_{n}=u_{n}^{-}+\alpha_{0}^{(n)}+u_{n}^{+}$de $A[[T]]\left[T^{-1}\right]$ du même type que $v$ ci-dessus en posant :

$$
u_{0}=u \text { et } u_{n+1}=u_{n} /_{\left(\alpha_{0}^{(n)}+u_{n}^{+}\right)}=1+u_{n}^{-}\left(\alpha_{0}^{(n)}+u_{n}^{+}\right)^{-1} .
$$

Soit $I_{n}^{+}$(resp. $\left.I_{n}^{-}\right)$l'idéal engendré par les coefficients de $u_{n}^{+}$(resp. $\left.u_{n}^{-}\right)$. Il 
est immédiat que $I_{n}^{-} \subseteq I_{0}^{-}$et que $I_{n+1}^{+} \subseteq I_{n}^{-} I_{n}^{+}$d'où $I_{n}^{+} \subseteq\left(I_{0}^{-}\right)^{n}$. Comme $I_{0}^{-}$ est un idéal nilpotent, car il est engendré par un nombre fini de nilpotents, on a $u_{n}^{+}=0$ pour $n$ assez grand, c.à.d. $u_{n}=u_{n}^{-}+\alpha_{0}^{(n)}$. Or par construction de la suite $i l$ existe $u^{\prime} \in A[[T]]^{*}$ tel que $u_{n}=u u^{\prime-1}=\alpha_{0}^{(n)} u^{\prime \prime}$, avec $u^{\prime \prime} \in T^{-1} A\left[T^{-1}\right]$ à coefficients dans $I_{0}^{-}$et $\alpha_{0}^{(n)} \in A^{*}$, d'où $u=u^{\prime} \cdot \alpha_{0}^{(n)} \cdot u^{\prime \prime} . C e$ qui donne le cas $C=A[[T]]$ immédiatement. L'unicité de la décomposition est triviale.

Cas général. L'hypothèse $C / T C \simeq A$ entraîne que $T A[[T]] \cap C=T C$. D'autre part on a $T \in C$. Il en résulte que tout élément $\bar{s} \in M=A[[T]] / C$ est uniquement divisible par $T$, c.à.d. il existe $\bar{s}^{\prime}$ unique tel que $\bar{s}=T \bar{s}^{\prime}$. Donc si $M_{T}=M \otimes_{C} C\left[T^{-1}\right]$ le $C$-morphisme naturel $M \longrightarrow M_{T}$ est un isomorphisme. On a alors le diagramme commutatif avec les lignes exactes :

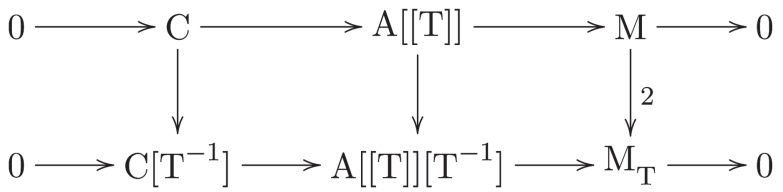

qui permet de prouver que $C\left[T^{-1}\right] \cap A[[T]]=C$. Soit $u \in C\left[T^{-1}\right]^{*} \subseteq$ $A[[T]]\left[T^{-1}\right]^{*}$.

On écrit $u^{\prime}=T^{n} u^{\prime} u^{\prime \prime}$. On a alors que $u^{\prime} \in C\left[T^{-1}\right]^{*} \cap A[[T]]$. D'après ce que l'on vient de voir $u^{\prime} \in C^{*}$ d'où on a ainsi montré le cas général.

Soit $\mathfrak{I} \subset \Im$ le $S$-sous-faisceau en groupes engendré par la section $T$ de $\Im$ donnée par un générateur $T$ de $I$ et par le $S$-sous-groupe $\mathfrak{I}^{0} \subset$ S. Le $S$ groupe $\mathfrak{J}$ est indépendant du choix de $T$.

Par définition de $\mathfrak{I}$, on a une suite exacte de $S$-groupes

$$
1 \longrightarrow \mathfrak{J}^{0} \longrightarrow \mathfrak{J} \longrightarrow \underline{\mathrm{Z}}_{S} \longrightarrow 0 \text {. }
$$

Le choix d'un générateur $T$ de $I$ donne lieu à un scindage de (0.9). D'où il résulte que $\mathfrak{J}$ est isomorphe au produit $\underline{Z}_{S} \times \mathfrak{J}^{0}$.

On définit un $S$-groupe par

$$
\check{W}=\Im / \mathfrak{J} .
$$

On a donc une suite exacte de $S$-groupes

$$
1 \longrightarrow \mathfrak{J} \longrightarrow \Im \longrightarrow \check{W} \longrightarrow 1 \text {. }
$$

Soient $B=A[[T]]$ et $A^{\prime}$ une $A$-algèbre. On définit le $S$-sous-foncteur en 
groupes $\check{W}_{T}$ de $\Im$ par

$$
\check{W}_{T}\left(A^{\prime}\right)=\left\{1+a_{-1}^{\prime} T^{-1}+\ldots+a_{-n}^{\prime} T^{-n} \mid a_{-1}^{\prime}, \ldots, a_{-n}^{\prime} \in \operatorname{Nil}\left(A^{\prime}\right)\right\} .
$$

(Nil $\left(A^{\prime}\right)$ désigne le nilradical de $A^{\prime}$.) Il est facile de voir que $\check{W}_{T}$ est en fait un $S$-sous-faisceau de Zariski de $\Im$. Le lemme (0.8), montre que l'on a un isomorphisme de $S$-foncteurs

$$
\Im \simeq \check{W}_{T} \times \mathfrak{J}
$$

qui entraîne que la suite exacte $(0.11)$ est scindée.

On définit un $S$-foncteur $\underline{\mathrm{G}}_{\mathrm{mS}}\left(\mathfrak{X}_{n}\right)$ en posant pour tout $S$-schéma $S^{\prime}$

$$
\underline{\mathrm{G}}_{\mathrm{mS}}\left(\mathfrak{X}_{n}\right)\left(S^{\prime}\right)=\Gamma\left(\mathfrak{X}_{n}^{\prime}, \mathcal{O}_{\mathfrak{X}_{n}^{\prime}}^{*}\right),
$$

où $\mathfrak{X}_{n}^{\prime}=\mathfrak{X}_{n} \times_{S} S^{\prime}$, et $\mathcal{O}_{\mathfrak{X}_{n}^{\prime}}^{*} \subset \mathcal{O}_{\mathfrak{X}_{n}^{\prime}}$ désigne le sous-faisceau des unités.

On pose

$$
\mathfrak{J}_{n}^{0}=\underline{\mathrm{G}}_{\mathrm{mS}}\left(\mathfrak{X}_{n}\right) .
$$

On a alors que $\mathfrak{J}^{0}$ (resp. $\mathfrak{J}_{n}^{0}$ ) est isomorphe au $S$-groupe des séries formelles inversibles : $S^{\prime} \longrightarrow \mathcal{O}_{S^{\prime}}[[T]]^{*}$ (resp. séries formelles inversibles tronquées à l'ordre $\left.n+1: S^{\prime} \longrightarrow\left(\mathcal{O}_{S^{\prime}}[[T]] /\left(T^{n+1}\right)\right)^{*}\right)$.

On a pour tout entier $n \geq 0$, un $S$-homomorphisme

$$
\mathfrak{J}^{0} \longrightarrow \mathfrak{I}_{n}^{0}
$$

donné par le $S$-homomorphisme de restriction $\underline{\mathrm{G}}_{\mathrm{mS}}(\mathfrak{X}) \longrightarrow \underline{\mathrm{G}}_{\mathrm{mS}}\left(\mathfrak{X}_{n}\right)$.

Soit

$$
\Im_{n}=\Im / \operatorname{Ker}\left(\mathfrak{J}^{0} \longrightarrow \mathfrak{J}_{n}^{0}\right)
$$

La suite exacte (0.9) donne lieu à une autre

$$
1 \longrightarrow \mathfrak{I}_{n}^{0} \longrightarrow \mathfrak{I}_{n} \longrightarrow \underline{Z}_{S} \longrightarrow 0 \text {. }
$$

Soit

$$
\Im_{n}=\Im / \operatorname{Ker}\left(\Im^{0} \longrightarrow \Im_{n}^{0}\right)
$$

La donnée d'un isomorphisme $B \simeq A[[T]]$ entraîne celle d'un isomorphisme de $S$-schémas

$(0.20) \quad \mathfrak{J}_{n}^{0} \simeq \operatorname{Spec}\left(A\left[a_{0}, \ldots, a_{n}\right]\left[a_{0}^{-1}\right]\right)\left(\operatorname{resp} . \mathfrak{J}^{0} \simeq \operatorname{Spec}\left(A\left[a_{i}\right]\left[a_{0}^{-1}\right](i \in \mathbb{N})\right).\right)$.

Si $m \geq n$, il existe un $S$-homomorphisme canonique $\mathfrak{I}_{m}^{0} \longrightarrow \mathfrak{J}_{n}^{0}$. On vérifie que la limite projective $\lim _{\overleftarrow{n}} \mathfrak{J}_{n}^{0}$ s'identifie à $\mathfrak{J}^{0}$. Pour tout $n \geq 0$ le 
homomorphisme canonique $\lim _{\overleftarrow{n}} \mathfrak{J}_{n}^{0} \longrightarrow \mathfrak{I}_{n}^{0}$ correspond à celui donné par (0.16).

On a alors que $\mathfrak{J}^{0}$ est un $S$-groupe pro-lisse. En composant (0.16) avec le $S$-homomorphisme canonique

$$
\underline{\mathrm{G}}_{\mathrm{mS}}\left(\mathfrak{X}_{n}\right)=\mathfrak{I}_{n}^{0} \longrightarrow \underline{\mathrm{G}}_{\mathrm{mS}}\left(\mathfrak{X}_{0}\right)=\underline{\mathrm{G}}_{\mathrm{mS}}
$$

on obtient un $S$-homomorphisme $\mathfrak{J}^{0} \longrightarrow \underline{\mathrm{G}}_{\mathrm{mS}}$.

Soit

$$
\wedge^{0}=\operatorname{Ker}\left(\mathfrak{J}^{0} \longrightarrow \underline{\mathrm{G}}_{\mathrm{mS}}\right) \quad\left(\operatorname{resp} . \wedge_{n}^{0}=\operatorname{Ker}\left(\mathfrak{I}_{n}^{0} \longrightarrow \underline{\mathrm{G}}_{\mathrm{mS}}\right)\right) .
$$

On a alors les décompositions canoniques en produit direct

$$
\mathfrak{J}^{0}=\underline{\mathrm{G}}_{\mathrm{mS}} \times \wedge^{0}\left(\text { resp. } \mathfrak{J}_{n}^{0}=\underline{\mathrm{G}}_{\mathrm{mS}} \times \wedge_{n}^{0}\right) .
$$

On vérifie facilement que $\wedge^{0}$ s'identifie à la limite projective $\lim _{\overleftarrow{n}} \wedge_{n}^{0}$.

La suite exacte (0.11) donne la suite exacte

$$
0 \longrightarrow \mathfrak{I}_{n} \longrightarrow \Im_{n} \longrightarrow \breve{W} \longrightarrow 0
$$

D'après (0.8) le choix d'un isomorphisme $B \simeq A[[T]]$ donne lieu à un isomorphisme de $S$-groupes

$(0.24) \quad \Im \simeq \check{W} \times \underline{Z}_{S} \times \underline{G}_{m S} \times \wedge^{0} \quad\left(\right.$ resp. $\left.\Im_{n} \simeq \check{W} \times \underline{Z}_{S} \times \underline{G}_{m S} \times \wedge^{0}, n \in \mathbb{N}\right)$

On a alors un $S$-homomorphisme

$$
\varepsilon: \Im \longrightarrow \underline{Z}_{S}
$$

tel que si $u \in \Im\left(A^{\prime}\right)$ s'écrit $u=T^{m} u^{\prime} u^{\prime \prime}$ ( $m \in Z$ ), grâce à (0.24), alors $\varepsilon(u)=m$. Il est facile de voir que $\varepsilon$ est indépendant du choix d'un isomorphisme $B \simeq A[[T]]$. On pose $\Im^{0}=\operatorname{Ker} \varepsilon$, et $\Im^{n}=\varepsilon^{-1}(\underline{n})$ pour toute section $\underline{n}$ de $\underline{Z}_{S}$. On a ainsi une suite exacte de $S$-groupes

(0.26) $1 \longrightarrow \Im^{0} \longrightarrow \Im \stackrel{\varepsilon}{\longrightarrow} \underline{Z}_{S} \longrightarrow 0\left(\right.$ resp. $\left.1 \longrightarrow \Im_{n}^{0} \longrightarrow \Im_{n} \stackrel{\varepsilon_{n}}{\longrightarrow} \underline{Z}_{S} \longrightarrow 0\right)$, où $\Im_{n}^{0}=\Im^{0} / \operatorname{Ker}\left(\Im^{0} \longrightarrow \Im_{n}^{0}\right)$. L'homomorphisme $\varepsilon$ est compatible avec l'homomorphisme $\mathfrak{J} \longrightarrow \underline{Z}_{S}$ donné par (0.9).

Il résulte de la définition de $\breve{W}_{T}$ (cf. (0.12)) que $\check{W}_{T}$ est isomorphe au complété formel du groupe des vecteurs de Witt universel introduit par P. Cartier dans [1], et que si $\wedge_{T}^{0}$ désigne le $S$-groupe défini comme dans (0.21) à partir de $B=A[[T]]$, alors $\breve{W}_{T}$ s'identifie au dual de Cartier de $\wedge_{T}^{0}$ (cf. loc. cit.). Soit

$$
\mathbb{E}_{S}^{(\infty)}=\operatorname{Spec}\left(A\left[a_{-i}\right]\left(i \in \mathbb{N}^{+}\right)\right)
$$




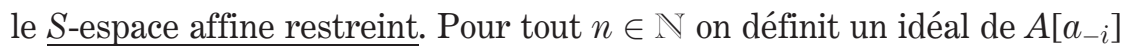
par

$$
K^{(n)}=\left(a_{-1}^{n}, \ldots, a_{-n}^{n}, a_{-n+1}, \ldots\right) .
$$

La famille d'idéaux $\left(K^{(n)}\right)(n \in \mathbb{N})$ est un système fondamental d'idéaux ouverts d'une unique topologie linéaire de $A\left[a_{-i}\right]^{\wedge}$. Soit $A\left[a_{-i}\right]^{\wedge}$ la $A$-algèbre topologique complétée de $A\left[a_{-i}\right]$. Il est clair que l'on a un isomorphisme de $S$-foncteurs

$$
\check{W}_{T} \simeq \widehat{\mathbb{E}}_{S}^{(\infty)}=\operatorname{Spf}\left(A\left[a_{-i}\right]^{\wedge}\left(i \in \mathbb{N}^{+}\right)\right) .
$$

Il résulte de (0.24) que le choix d'un isomorphisme $B \simeq A[[T]]$ entraîne un $S$-isomorphisme de groupes

$$
\check{W} \simeq \check{W}_{T},
$$

donc un isomorphisme de $S$-foncteurs $\breve{W} \simeq \widehat{\mathbb{E}}_{S}^{(\infty)}$.

Soit $I_{A} \subset B \widehat{\otimes}_{A} B$ l'idéal de définition de la section diagonale $\Delta: \mathfrak{X} \longrightarrow \mathfrak{X} \times_{S} \mathfrak{X}$. Soit $d$ un générateur de $I_{\Delta}$. L'image de $d$ dans $B_{\left\{B\left[I^{-1}\right]\right\}}=B \widehat{\otimes}_{A} B\left[I^{-1}\right]$, que l'on note toujours $d$, est un élément inversible. En effet, notons que si $B=A[[T]]$ on a alors $B \widehat{\otimes}_{A} B\left[I^{-1}\right] \simeq$ $A[[t]]\left[t^{-1}\right][[T]]$ en notant par $T$ (resp. $t$ ) l'élément $T \otimes 1$ (resp. $1 \otimes T$ ) de $A[[T]] \widehat{\otimes}_{A} A[[T]]\left[T^{-1}\right]$. De même on a $B \widehat{\otimes}_{A} B \simeq A[[t, T]]$. Il est facile de voir que

$$
I_{\Delta}=(t-T),
$$

d'où il existe $u \in A[[t, T]]^{*}$ tel que $d=u(t-T)$. On a dans $A[[t]]\left[t^{-1}\right][[T]]$ la relation

$$
(t-T)^{-1}=t^{-1}\left(1+\sum_{i \geq 1} t^{-i} T^{i}\right),
$$

qui donne que $d$ est inversible dans $A[[t]]\left[t^{-1}\right][[T]]$ et entraîne l'affirmation en général. Soit $d_{\diamond}$ l'image de $d$ par le $A$-homomorphisme

$$
B \widehat{\otimes}_{A} B \longrightarrow B
$$

obtenu par passage au quotient par l'idéal $(1 \otimes I)\left(B \widehat{\otimes}_{A} B\right)$.

Notation 0.31. (i) - Soit $\varphi_{d}^{0}$ la section de $\mathfrak{J}^{0}=\underline{\mathrm{G}}_{\mathrm{mS}}(\mathfrak{X})$ au-dessus de $\mathfrak{U}=\operatorname{Spec}\left(B\left[I^{-1}\right]\right)$ donnée par

$$
d^{-1} \in\left(B \widehat{\otimes}_{A} B\left[I^{-1}\right]\right)^{*}=\underline{\mathrm{G}}_{\mathrm{mS}}(\mathfrak{X})(\mathfrak{H})=\mathfrak{J}^{0}(\mathfrak{H}) .
$$


(ii) - L'élément $\left(d_{\circ} \otimes 1\right) d^{-1}$ donne lieu à une section de $\Im=\underline{\mathrm{G}}_{\mathrm{mS}}(\mathfrak{H})$ au-dessus de $\mathfrak{U}$, qui appartient en fait au $S$-sous-groupe $\mathfrak{J} \subset \Im$ ( $\mathfrak{I}$ est engendré par la section $d_{\circ} \otimes 1$ et par $\left.\mathfrak{J}^{0}\right)$, que l'on désigne par $\varphi_{d}^{1}$.

(iii) - Soit $\varphi_{T}^{0}$ la section de $\mathfrak{J}^{0}$ au-dessus de $\mathfrak{U}$ donnée par $(t-T)^{-1} \in B \widehat{\otimes}_{A} B\left[I^{-1}\right]=\mathfrak{J}^{0}(\mathfrak{H})$. Soit $\varphi_{T}^{1}$ la section de $\mathfrak{J}$ au-dessus de $\mathfrak{U}$ donnée par $T(t-T)^{-1}$. Si l'on pose $d=t-T$ on a $\varphi_{d}^{0}=\varphi_{T}^{0}$ et $\varphi_{d}^{1}=-\varphi_{T}^{1}$.

(La considération de $\varphi_{d}^{0}\left(r e s p . \varphi_{d}^{1}\right)$ pour un générateur d quelconque de $I_{\Delta}$ est imposée par les constructions géométriques du §1.)

Lemme 0.32. Avec les notations de (0.31), on a :

(i) - La classe $\varphi^{0}$ de $\varphi_{d}^{0}$ dans $\mathfrak{I}^{0}(\mathfrak{H}) / \mathfrak{J}^{0}(\mathfrak{X})$ est indépendante du choix de $d$ et coïncide avec celle de $\varphi_{T}^{0}$.

(ii) - La classe $\varphi^{1}$ de $-\varphi_{d}^{1}$ dans $\mathfrak{I}(\mathfrak{U}) / \operatorname{Ker}\left(\mathfrak{J}^{0}(\mathfrak{X}) \longrightarrow \mathfrak{J}^{0}(S)\right)$ est indépendante du choix de d et coïncide avec celle de $\varphi_{T}^{1}$.

PReuve. Soit d' un autre générateur de $I_{\Delta}$. Il existe alors $u \in\left(B \widehat{\otimes}_{A} B\right)^{*}$ tel que $d^{\prime}=u d$. Notons par $d_{\circ}^{\prime}\left(r e s p . d_{\circ}, u_{\circ}\right)$ l'image de $d^{\prime}(r e s p . d, u)$ par (0.30). On a alors

$$
\left(d_{\circ}^{\prime} \widehat{\otimes} 1\right) d^{\prime-1}=\left(u_{\circ} \otimes 1\right) u^{-1}\left(d_{\circ} \otimes 1\right) d^{-1} .
$$

Il est clair que l'image de $\left(u_{\circ} \otimes 1\right) u^{-1}$ par $(0.30)$ est égale à 1 . Il en résulte (ii), compte tenu de (0.30). La preuve de (i) est analogue.

C.Q.F.D.

Proposition 0.33. On reprend les notations de (0.31). Soit $T$ un générateur de I. On suppose $S$ noethérien. Soit

$$
\prod^{n+2} \varphi_{T}^{0}: \prod^{n+2} \mathfrak{U} \longrightarrow \mathfrak{J}_{n}^{0}
$$

le S-morphisme obtenu en composant le S-morphisme $\prod^{n+2} \mathfrak{u} \longrightarrow \mathfrak{J}^{0}$ faisant correspondre à une section $\left(x_{1}, \ldots, x_{n+2}\right)$ de $\prod^{n+2} \mathfrak{u}$ l'élément

$$
\varphi_{T}^{0}\left(x_{1}\right) \ldots \varphi_{T}^{0}\left(x_{n+2}\right) \in \mathfrak{J}^{0}\left(\prod^{n+2} \mathfrak{U}\right)
$$

avec le $S$-morphisme canonique $\mathfrak{J}^{0} \longrightarrow \mathfrak{I}_{n}^{0}(c f .(0.16))$.

On a alors que $\prod^{n+2} \varphi_{T}^{0}$ est un S-morphisme fidlement plat. 
Preuve. Soit $\sigma_{j}(1 \leq j \leq n+2)$ la j-ème fonction symétrique de $\left(t_{1}, \ldots, t_{n+2}\right)$, c.̀̀.d.

$$
\sigma_{j}=\sum t_{\alpha_{1}} \ldots t_{\alpha_{j}}\left(1 \leq \alpha_{1}<\ldots<\alpha_{j} \leq n+2\right)
$$

On pose

$\left(t_{1}-T\right)\left(t_{2}-T\right) \ldots\left(t_{n+2}-T\right)=a_{0}+a_{1} T+\ldots+a_{n+1} T^{n+1}+(-1)^{n+2} T^{n+2}$, ce qui donne :

$$
a_{n+2-j}=(-1)^{n+2-j} \sigma_{j} \quad(1 \leq j \leq n+2) .
$$

Le A-homomorphisme

$$
A\left[a_{0}, \ldots, a_{n+1}\right] \longrightarrow A\left[t_{1}, \ldots, t_{n+2}\right]
$$

défini par les équations (*) est fidèlement plat. Ce qui résulte du fait bien connu que $A\left[t_{1}, \ldots, t_{n+2}\right]$ est un $A\left[\sigma_{1}, \ldots, \sigma_{n+2}\right]$-module libre de type fini.

Il en résulte que le A-homomorphisme induit

$$
A\left[a_{0}, \ldots, a_{n+1}\right]\left[a_{0}^{-1}\right] \longrightarrow A\left[t_{1}, \ldots, t_{n+2}\right]\left[t_{1}^{-1}, \ldots, t_{n+2}^{-1}\right]
$$

est aussi fidèlement plat.

Soit $g=\left(\prod^{n+2} \varphi_{T}^{0}\right)^{-1}$. Il est clair que le A-homomorphisme correspondant à $g$ :

$$
A\left[a_{0}, \ldots, a_{n}\right]\left[a_{0}^{-1}\right] \longrightarrow A\left[\left[t^{-1}\right]\right]\left[t_{1}^{-1}\right] \otimes_{A} \ldots \otimes_{A} A\left[\left[t_{n+2}\right]\right]\left[t_{n+2}^{-1}\right]
$$

est obtenu en composant (**) avec le A-homomorphisme plat

$$
A\left[a_{0}, \ldots, a_{n}\right]\left[a_{0}^{-1}\right] \longrightarrow A\left[a_{0}, \ldots, a_{n+1}\right]\left[a_{0}^{-1}\right]
$$

à gauche, et avec le A-homomorphisme plat (c'est ici que l'hypothèse A noethérien intervient).

$A\left[t_{1}\right]\left[t_{1}^{-1}\right] \otimes_{A} \ldots \otimes_{A} A\left[t_{n+2}\right]\left[t_{n+2}^{-1}\right] \longrightarrow A\left[\left[t_{1}\right]\right]\left[t_{1}^{-1}\right] \otimes_{A} \ldots \otimes_{A} A\left[\left[t_{n+2}\right]\left[t_{n+2}^{-1}\right]\right.$ à droite. On a ainsi que g est un A-morphisme plat. Montrons que g est surjectif. On est alors ramené à montrer que pour tout corps algébriquement clos $k$, qui soit une A-algèbre, le $k$-morphisme fibre $g_{k}$ de g audessus de Spec (k) est surjectif.

Notons par $g_{k}^{\prime}$ le k-morphisme

$$
\operatorname{Spec}\left(k\left(\left(t_{1}\right)\right) \otimes \ldots \otimes_{k} k\left(\left(t_{n+2}\right)\right)\right) \longrightarrow \operatorname{Spec}\left(k\left[a_{0}, \ldots, a_{n}\right]\left[a_{0}^{-1}\right]\right)
$$

défini à partir des équations (*). On voit facilement que $g_{k}^{\prime}$ se factorise à travers $g_{k}$, et qu'il est un k-morphisme plat. Il nous suffit de montrer que $g_{k}^{\prime}$ 
est surjectif. Soit $g^{\prime \prime}{ }_{k}$ le k-morphisme

$$
\operatorname{Spec}\left(k\left(t_{1}\right) \otimes_{k} \ldots \otimes k\left(t_{n+2}\right)\right) \longrightarrow \operatorname{Spec}\left(k\left[a_{0}, \ldots, a_{n}\right]\left[a_{0}^{-1}\right]\right)
$$

défini à partir des équations (*). Puisque $g_{k}^{\prime}$ se factorise par $g^{\prime \prime}{ }_{k}$ et que

$$
k\left(t_{1}\right) \otimes_{k} \ldots \otimes k\left(t_{n+2}\right) \longrightarrow k\left(\left(t_{1}\right)\right) \otimes_{k} \ldots \otimes_{k} k\left(\left(t_{n+2}\right)\right)
$$

est fidèlement plat on est ramené à montrer que $g^{\prime \prime}{ }_{k}$ est surjectif.

Il suffit de voir que pour tout $\left(c_{0}, \ldots, c_{n}\right) \in k^{n+1}$ avec $c_{0} \neq 0$, le système d'équations

$(* * *)$

$$
(-1)^{j} \sigma_{n+2-j}-c_{j}=0 \quad(0 \leq j \leq n)
$$

admet une solution $\left(x_{1}, \ldots, x_{n+2}\right)$ dans une extension (finie) $K$ du corps $k(u)$ des fractions rationnelles en l'indéterminée $u$, dont toute les composantes soient transcendantes sur $k$.

Soit $K$ une extension finie de $k(u)$ contenant toutes les racines $x_{1}, \ldots, x_{n+2}$ du polynôme

$$
P(T)=\left(c_{0}+c_{1} T+\ldots+c_{n} T^{n}+u T^{n+1}+T^{n+2}\right) .
$$

Le lemme (0.34) ci-dessus montre bien que le point $\left(x_{1}, \ldots, x_{n+2}\right)$ fourni une solution de $(* * *)$ avec la propriété voulue. On a ainsi que $g^{\prime \prime}{ }_{k}$ est un morphisme surjectif.

C.Q.F.D.

Lemme 0.34. Soit $t$ un élément algébrique sur $k$ différent de 0 . Alors $P(t)=c_{0}+c_{1} t+\ldots+c_{n} t^{n}+u t^{n+1}+t^{n+1}$ est un élément transcendant sur $k$.

Preuve. Quitte à substituer le polynôme $P(T)$ par une puissance $P(T)^{p i}(i \in \mathbb{N})$ où $p$ est la caractéristique de $k$, on peut supposer que $t$ est séparable sur $k$. Soit $k^{\prime}$ une extension algébrique de $k$ qui contient toutes les racines $t_{1}, \ldots, t_{d} d u$ polynôme minimal de $t$ (de degré $d$ ). Supposons que $P\left(t_{1}\right), \ldots, P\left(t_{d}\right)$ sont algébriques sur $k$. Il existe un polynôme $Q(T) \in k[u][T]$ de degré en $T \leq d$ tel que :

(a) $-Q\left(t_{1}\right)=P\left(t_{1}\right), \ldots Q\left(t_{d}\right)=P\left(t_{d}\right)$;

(b) - les coefficients de $Q(T)$ sont des polynômes en u de degré $\leq 1$, et il existe au moins un coefficient de $Q(T)$ qui n'appartient pas à $k$.

L'hypothèse de séparabilité de $t$ donne que la matrice $\left[t_{i}^{j}\right]$ $(1 \leq i \leq d, 0 \leq j<d)$ est de rang $d$. Il en résulte que l'on peut exprimer $u$ comme une combinaison linéaire dans $k$ des éléments algébriques sur $k$ $P\left(t_{1}\right), \ldots, P\left(t_{d}\right)$ ce qui est absurde. 
On procède à définir deux sections $\gamma^{0} \in \check{W}(\mathfrak{X})$ et $\gamma^{1} \in\left(\check{W} \times \underline{Z}_{S}\right)(\mathfrak{X})$ correspondant respectivement à $\varphi^{0} \in \mathfrak{J}^{0}(\mathfrak{U}) / \mathfrak{J}^{0}(\mathfrak{X})$ et à $\varphi^{1} \in \mathfrak{J}(\mathfrak{l}) /$ $\operatorname{Ker}\left(\mathfrak{I}^{0}(\mathfrak{X}) \longrightarrow \mathfrak{I}^{0}(S)\right)$ comme l'on verra dans $\S 1$.

Notation 0.35. (i) - Soit $d \in I_{\Delta} \subset B \widehat{\otimes}_{A} B$ (resp. $d_{0}$ ) un générateur d'idéal de définition $I_{\Delta}$ de la diagonale de $\mathfrak{X} \times_{S} \mathfrak{X}$ (resp. défini comme dans $(0.30)$ ). Notons par $d_{n}$ l'image de d dans $B \widehat{\otimes}_{A} B_{n}(n \geq 0)$. On vérifie facilement que $d_{n}$ (resp. $\left.\left(d_{0} \otimes 1\right)^{-1} d_{n}\right)$ est une unité de $B_{\left\{B_{n}\right\}}\left(I_{\left\{B_{n}\right\}}^{-1}\right)$, c.à.d.

$$
d_{n}\left(\operatorname{resp} .\left(d_{0} \otimes 1\right)^{-1} d_{n}\right) \in\left(B_{\left\{B_{n}\right\}}\left[I_{\left\{B_{n}\right\}}^{-1}\right]\right)^{*} .
$$

(ii) - Soit

$$
\gamma_{d_{n}}^{0}\left(\operatorname{resp} \cdot \gamma_{d_{n}}^{1}\right) \in \Gamma\left(\mathfrak{X}_{n}, \underline{\mathbb{G}}_{m S}(\mathfrak{U})\right)=\left(B_{\left\{B_{n}\right\}}\left[I_{\left\{B_{n}\right\}}^{-1}\right]\right)^{*}
$$

la section donnée par $\left(d_{0} \otimes 1\right)^{-1} d_{n}\left(\right.$ resp. $\left.d_{n}\right)$. On a que $\gamma_{d_{n}}^{0}$ est en fait une section de $\Im^{0} \subset \Im=\underline{G}_{m S}(\mathfrak{l})$.

Soient $n, m \in \mathbb{N} n \geq m$. On vérifie que la restriction de $\gamma_{d_{n}}^{0}\left(\right.$ resp. $\left.\gamma_{d_{n}}^{1}\right) \grave{a}$ $\mathfrak{X}_{m} \longrightarrow \mathfrak{X}_{n}$ est égale $\gamma_{d_{m}}^{0}\left(\right.$ resp. $\gamma_{d_{m}}^{1}$ ). Soit

$$
\gamma_{d}^{0}: \mathfrak{X} \longrightarrow \Im^{0}\left(\text { resp. } \gamma_{d}^{1}: \mathfrak{X} \longrightarrow \Im\right)
$$

le S-morphisme (de S-foncteurs) donné par le système $\left(\gamma_{d_{n}}^{0}\right)\left(\right.$ resp. $\left.\left(\gamma_{d_{n}}^{1}\right)\right)$.

Noter que $\mathfrak{X}=\lim _{\vec{n}} \mathfrak{X}_{n}$.

Si $B=A[[T]]$ alors $B_{n} \simeq A[[t]] /\left(t^{n+1}\right)$.

Soit $d=T-t$. On a alors $\gamma_{d_{n}}^{0}=1-\frac{t}{T}($ resp. $T-t) \in\left(A[[t]] /\left(t^{n+1}\right)\right)[[T]]\left[T^{-1}\right]^{*}$.

Noter que

$$
\left(\gamma_{d_{n}}^{0}\right)^{-1}=1+\frac{t}{T}+\ldots+\frac{t^{n}}{T^{n}}\left(\operatorname{resp} .\left(\gamma_{d_{n}}^{1}\right)^{-1}=T^{-1}\left(1+\frac{t}{T}+\ldots+\frac{t^{n}}{T^{n}}\right)\right) .
$$

Soit U un générateur de I. On désigne par $\gamma_{u}^{0}\left(\right.$ resp. $\left.\gamma_{u}^{1}\right)$ la section de $\Im^{0}$ (resp. S) au-dessus de $\mathfrak{X}$ donnée par $1-u U^{-1}($ resp. $U-u) \in$ $\left(B \widehat{\otimes}_{A} B\right)\left[U^{-1}\right]^{*}$, où l'on désigne $U \otimes 1$ par $U$ et $1 \otimes U$ par $u$. Si $d=U-u$ on a alors $\gamma_{d}^{0}=\gamma_{u}^{0}\left(\right.$ resp. $\left.\gamma_{d}^{1}=\gamma_{u}^{1}\right)$.

(iii) - On montre comme dans (0.32) que la classe de $\gamma_{d}^{0}$ (resp. $\left.\gamma_{d}^{1}\right)$ dans

$$
\Im^{0}(\mathfrak{X}) / \operatorname{Ker}\left(\mathfrak{I}^{0}(\mathfrak{X}) \longrightarrow \mathfrak{I}^{0}(S)\right)\left(\operatorname{resp} . \Im(\mathfrak{X}) / \mathfrak{J}^{0}(\mathfrak{X})\right)
$$

est indépendante du choix de d. Notons par

$$
\gamma^{0}: \mathfrak{X} \longrightarrow \breve{W}\left(\text { resp. } \gamma^{1}: \mathfrak{X} \longrightarrow \Im / \mathfrak{J}^{0}=\underline{Z}_{S} \times \check{W}\right)
$$


le S-morphisme donné par l'image de $\gamma_{d}^{0}\left(\right.$ resp. $\left.\gamma_{d}^{1}\right)$ dans

$$
\Im^{0}(\mathfrak{X}) / \mathfrak{J}^{0}(\mathfrak{X})=\check{W}(x)\left(\operatorname{resp} . \Im(\mathfrak{X}) / \mathfrak{J}^{0}(\mathfrak{X})\right) .
$$

REMARQUe 0.36. Les constructions de $\mathfrak{J}^{0}$, $\mathfrak{I}, \Im, \Im^{0}$ (resp. $\mathfrak{J}_{n}^{0}, \mathfrak{J}_{n}, \Im_{n}$, $\left.\Im_{n}^{0}\right)$ ainsi que celle de $\varphi^{0}, \varphi^{1}, \gamma^{0}, \gamma^{1}$ sont fonctorielles vis à vis des isomorphismes de A-algèbres adiques augmentées isomorphes à $A[[T]]$.

On a plus précisément, avec les notations de (0.31) et de (0.35), le résultat suivant. Soient $B^{\prime} \simeq B$ un $A$-isomorphisme et $d^{\prime}$ un générateur de $I_{A} \subset B^{\prime} \widehat{\otimes}_{A} B^{\prime}$. Alors l'image d de d' par $B^{\prime} \widehat{\otimes}_{A} B^{\prime} \simeq B \widehat{\otimes}_{A} B^{\prime}$ est un générateur de $I_{\Delta} \subset B \widehat{\otimes}_{A} B$ et la section $\gamma_{d}^{0}$ (resp. $\varphi_{d}^{1}, \gamma_{d}^{0}, \gamma_{d}^{1}$ ) correspond fonctoriellement à la section $\gamma_{d^{\prime}}^{0}\left(\operatorname{resp} . \varphi_{d^{\prime}}^{1}, \gamma_{d^{\prime \prime}}^{0}, \gamma_{d^{\prime}}^{1}\right)$.

Soit $B=A[[T]]$. Il résulte du lemme (0.7) l'existence d'un scindage

$$
\breve{W} \longrightarrow \Im^{0}
$$

de la suite exacte

$$
1 \longrightarrow \mathfrak{J}^{0} \longrightarrow \Im^{0} \longrightarrow \check{W} \longrightarrow 1
$$

dont l'image est égale à $\check{W}_{T}$ (cf. (0.12)). On obtient alors un relèvement

$$
\bar{\gamma}^{0}: \mathfrak{X} \longrightarrow \breve{W} \longrightarrow \Im^{0}
$$

de $\gamma^{0}: \mathfrak{X} \longrightarrow \breve{W}$, qui s'avère être égal à la section $\gamma_{T}^{0}$ (cf. (0.35),(ii)) de $\Im$, donnée par $1-t T^{-1}$. On a ainsi que le morphisme

$$
\gamma_{T}^{0}: \mathfrak{X}=\operatorname{Spf}(A[[T]]) \longrightarrow \check{W}_{T} \subset \Im^{0}
$$

de $\mathfrak{X}$ dans le complété formel du groupe des vecteurs de Witt coïncide avec le morphisme noté $[t]$ dans [1]. Il résulte alors de loc. cit. la

Proposition 0.39. Soient $S, \mathfrak{x}, \gamma^{0}, \breve{W}$ définis comme ci-dessus. Soit $G$ un $S$-schéma en groupes commutatif et lisse. Le couple $\left(\breve{W}, \gamma^{0}\right)$ vérifie la propriété universelle suivante: "La flèche

$$
\operatorname{Hom}_{S-g r}(\check{W}, G) \longrightarrow \operatorname{Ker}(G(\mathfrak{X}) \longrightarrow G(S))
$$

induite par composition avec $\gamma^{0}$ est bijective".

Soit $\operatorname{Sym}^{(n)}(\mathfrak{X})$ la puissance symétrique $n$-ème de $\mathfrak{X}$, i.e. le quotient du produit $n$-ème $\mathfrak{X} \times_{S} \ldots \times_{S} \mathfrak{X}$ par l'action du groupe symétrique $\mathfrak{S}_{n}$. On a que $\operatorname{Sym}^{(n)}(\mathfrak{X})$ est représentable par un $S$-schéma formel isomorphe au spectre formel $\operatorname{Spf}\left(A\left[\left[a_{-1}, \ldots, a_{-n}\right]\right]\right)$, de l'anneau adique des séries 
formelles

$$
A\left[\left[a_{-1}, \ldots, a_{-n}\right]\right] \text { en } a_{-1}, \ldots, a_{-n},
$$

à coefficients dans $A$.

La multiplication de $\check{W}$ donne lieu à un $S$-morphisme

$$
\operatorname{Sym}^{(n)}\left(\gamma^{0}\right): \operatorname{Sym}^{(n)}(\mathfrak{X}) \longrightarrow \check{W},
$$

qui est en fait une immersion fermée de $S$-schémas formels. En effet on vérifie, si $\mathfrak{X}=\operatorname{Spf}(A[[T]])$, que l'image de $\operatorname{Sym}^{(n)}\left(\gamma_{T}^{0}\right): \operatorname{Sym}^{(n)}(\mathfrak{X}) \longrightarrow \check{W}_{T}$ est donnée par le sous-foncteur de $\breve{W}_{T}$ dont les sections $1+a_{-1} T^{-1}+\ldots+a_{-m} T^{-m}$ vérifient $a_{-n-1}=\ldots=a_{-m}=0$. Le $n$-ème $\mathfrak{X}^{n}=\mathfrak{X} \times_{S} \ldots \times_{S} \mathfrak{X}$ s'identifie à $\operatorname{Spf}\left(A\left[\left[t_{1}, \ldots, t_{n}\right]\right]\right)$ et le morphisme $\mathfrak{X}^{n} \longrightarrow \operatorname{Sym}^{(n)}(\mathfrak{X})$ correspond au $A$-homomorphisme de $A$-algèbres

$$
A\left[\left[a_{-1}, \ldots, a_{-n}\right]\right] \longrightarrow A\left[\left[t_{1}, \ldots, t_{n}\right]\right]
$$

faisant correspondre $(-1)^{i} \sigma_{i}$ à $a_{-i}$, où $\sigma_{i}$ désigne la $i$-ème fonction symétrique de $t_{1}, \ldots, t_{n}$.

On identifie $\operatorname{Sym}^{(n)}(\mathfrak{X})$ à son image dans $\check{W}$ et l'on pose

$$
W_{n}=\operatorname{Sym}^{(n)}(\mathfrak{X}) .
$$

Pour tout couple d'entiers $1 \leq m \leq n$, on a un morphisme

$$
\operatorname{Sym}^{(m)}(\mathfrak{X}) \longrightarrow \operatorname{Sym}^{(n)}(\mathfrak{X}),
$$

qui correspond au $A$-homomorphisme $A\left[\left[a_{-1}, \ldots, a_{-n}\right]\right] \longrightarrow A\left[\left[a_{-1}, \ldots, a_{-m}\right]\right]$ obtenu par passage au quotient par l'idéal engendré par $a_{-m-1}, \ldots, a_{-n}$. Ce morphisme se déduit par passage au quotient du morphisme $\mathfrak{X}^{m} \longrightarrow \mathfrak{X}^{n}$ donné par le $A$-homomorphisme $A\left[\left[t_{1}, \ldots, t_{n}\right]\right] \longrightarrow A\left[\left[t_{1}, \ldots, t_{m}\right]\right]$ envoyant $t_{i}$ sur 0 pour $i>m$.

Étant donnés des entiers $0 \leq m \leq n \leq p$ on a un diagramme commutatif :

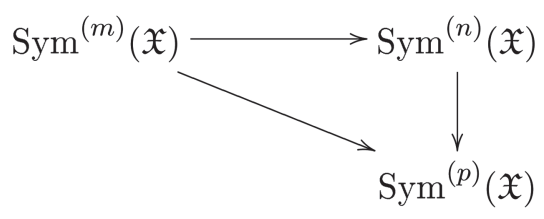

On a ainsi un système inductif $\left(W_{n}\right)$ de $S$-schémas formels.

Notons par $\underset{n}{\lim } W_{n}$ la limite inductive prise dans la catégorie des $S$ faisceaux de Zariski. On a alors 
Proposition 0.44. Avec les notations ci-dessus on a que les inclusions $W_{n} \longrightarrow \breve{W}$ induisent un isomorphisme de S-faisceaux de Zariski

$$
\underset{\vec{n}}{\lim _{n}} W_{n} \simeq \check{W}
$$

Notation 0.45. Soient $n, m \geq 0$ des entiers, et $\underline{v}$ une section de $\underline{Z}_{S}$ audessus d'un S-schéma $S^{\prime}$.

(i) - La suite exacte (0.11) donne lieu aux suites exactes

$$
1 \longrightarrow \mathfrak{J}^{0} \longrightarrow \Im^{0} \longrightarrow \check{W} \longrightarrow 1
$$

et

$$
1 \longrightarrow \Im_{n}^{0} \longrightarrow \Im_{n}^{0} \longrightarrow \breve{W} \longrightarrow 1 \text { (cf. (0.26) et }(0.23) \text { ). }
$$

Soit ${ }^{(m)} \Im^{0}$ (resp. ${ }^{(m)} \Im_{n}^{0}$ ) le $\mathfrak{J}^{0}$-torseur (resp. $\mathfrak{J}_{n}^{0}$-torseur) au-dessus de $W_{m}$ obtenu à partir de la première (resp. deuxième) suite exacte ci-dessus et du morphisme $W_{m} \longrightarrow \breve{W}$.

(ii) - Soit ${ }^{(m)} \Im$ (resp. ${ }^{(m)} \Im_{n}$ ) le $\mathfrak{J}$-torseur (resp. $\mathfrak{I}_{n}$-torseur) obtenu à partir de la suite exacte $1 \longrightarrow \Im^{0} \longrightarrow \Im \longrightarrow \breve{W} \longrightarrow 1$ (cf. (0.11)) et du morphisme $W_{m} \longrightarrow \breve{W}$.

(iii) - Soit $\Im^{\underline{v}}=\varepsilon^{-1}(\underline{v})\left(\right.$ resp. $\left.\Im_{\bar{n}}^{\underline{v}}=\varepsilon_{n}^{-1}(\underline{v})\right)(c f .(0.26))$. On a un $S^{\prime}$-morphisme $\Im^{\underline{\underline{y}}} \longrightarrow \check{W}_{S^{\prime}}\left(\right.$ resp. $\left.\Im^{\underline{\underline{v}}} \longrightarrow \check{W}_{S^{\prime}}\right)$ qui fait de $\Im^{\underline{\underline{v}}}$ (resp. $\left.\Im_{n}^{\underline{v}}\right)$ un $\mathfrak{J}_{S^{\prime}}^{0}$-torseur (resp. $\left(\mathfrak{J}_{n}^{0}\right)_{S^{\prime}}$ torseur). Notons par ${ }^{(m)} \mathfrak{\Im}^{\underline{\underline{p}}}\left(\right.$ resp. $\left.{ }^{(m)} \mathfrak{J}_{n}^{\underline{\nu}}\right)$ le $\mathfrak{J}^{0}$ (resp. $\left.\mathfrak{J}_{n}^{0}\right)$ torseur image inverse par $\left(W_{m}\right)_{S^{\prime}} \longrightarrow \check{W}_{S^{\prime}}$.

La suite exacte $(0.11)$ (resp. $1 \longrightarrow \mathfrak{J}^{0} \longrightarrow \Im^{0} \longrightarrow \check{W} \longrightarrow 1$ (cf. (0.45), (i))) nous permet de regarder $\Im$ (resp. $\Im^{0}$ ) comme un $\mathfrak{J}$-torseur (resp. $\mathfrak{J}^{0}$-torseur) au dessus de $\breve{W}$. La donnée d'un générateur $U$ de $I$ entraîne un isomorphisme de $A$-algèbre adique augmentées $A[[T]] \simeq B$. Par fonctorialité (cf. (0.36)) on en déduit un scindage

$$
\sigma_{U}: \check{W} \longrightarrow \Im^{0}
$$

de la suite exacte

$$
1 \longrightarrow \mathfrak{J}^{0} \longrightarrow \Im^{0} \longrightarrow \check{W} \longrightarrow 1
$$

et un relèvement

$$
\gamma_{U}^{0}: \mathfrak{X} \longrightarrow \Im^{0} \text { de } \gamma^{0}: \mathfrak{X} \longrightarrow \breve{W}
$$

à partir de celui de la suite exacte (0.38) tel que

$$
\gamma_{U}^{0}=(U \otimes 1-1 \otimes U)(U \otimes 1)^{-1} \in\left(\left(B \widehat{\otimes}_{A} B\right)\left[(U \otimes 1)^{-1}\right]\right)^{*}=\Im(\mathfrak{X}) .
$$


Soient $U$ et $V$ des générateurs de $I$. On a alors

$$
\begin{aligned}
& \gamma_{V}^{0}\left(\gamma_{U}^{0}\right)^{-1}=(V \otimes 1-1 \otimes V)(V \otimes 1)^{-1}(U \otimes 1-1 \otimes U)^{-1}(U \otimes 1) \in \\
&\left(B \widehat{\otimes}_{A} B\right)^{*}=\mathfrak{J}^{0}(\mathfrak{X})
\end{aligned}
$$

En fait $\gamma_{V}^{0}\left(\gamma_{U}^{0}\right)^{-1}$ est une section de $\operatorname{Ker}\left(\mathfrak{J}^{0}(\mathfrak{X}) \longrightarrow \mathfrak{J}^{0}(S)\right)$.

Noter que l'ensemble des isomorphismes de $A$-algèbres adiques augmentées $\operatorname{Isom}(A[[T]], B)$ de $A[[T]]$ avec $B$, s'identifie à l'ensemble des générateurs de $I$. Notons par $\operatorname{Aut}(B)$ les $A$-automorphismes de la $A$-algèbre adique $B$. On a alors que $\operatorname{Aut}(B)$ opère $\operatorname{sur} \operatorname{Isom}(A[[T]], B)$, qui devient alors un $\operatorname{Aut}(B)$ torseur à gauche.

On définit un $S$-homomorphisme $h(U, V): \check{W} \longrightarrow \mathfrak{J}^{0} \operatorname{par} h(U, V) \sigma_{U}=\sigma_{V}$.

Notation 0.47. Notons par ${ }^{(m)} \sigma_{U}$ (resp. $\left.{ }^{(m)} \sigma_{U_{n}}\right)$ le scindage de ${ }^{(m)} \Im^{0} \longrightarrow W_{m}$ (resp. ${ }^{(m)} \Im_{n}^{0} \longrightarrow W_{m}$ ) induit par $\sigma_{U}$. On pose

$$
\begin{aligned}
\left.{ }^{(m)} h(U, V)={ }^{(m)} \sigma_{V}{ }^{\left({ }^{(m)}\right.} \sigma_{U}\right)^{-1} \in \Gamma\left(W_{m}, \mathfrak{J}^{0}\right) \\
\\
\left(\operatorname{resp} \cdot{ }^{(m)} h(U, V)_{n}={ }^{(m)} \sigma_{V_{n}}\left({ }^{(m)} \sigma_{U_{n}}\right)^{-1} \in \Gamma\left(W_{m}, \mathfrak{J}_{n}^{0}\right)\right) .
\end{aligned}
$$

D'où

$$
{ }^{(1)} h(U, V)=h(U, V) \circ \gamma^{0}=\gamma_{V}^{0}\left(\gamma_{U}^{0}\right)^{-1} \in \Gamma\left(\mathfrak{X}, \mathfrak{J}^{0}\right),
$$

et en général

$$
{ }^{(m)} h(U, V)=h(U, V) \circ \operatorname{Sym}^{(n)}\left(\gamma^{0}\right) .
$$

Noter que ${ }^{(m)} h(U, V): \operatorname{Sym}^{(m)}(\mathfrak{X}) \longrightarrow \mathfrak{J}^{0}$ s'obtient par passage au quotient à partir de

$$
\prod^{m}{ }^{(1)} h(U, V): \prod^{m} \mathfrak{X} \longrightarrow \mathfrak{J}^{0},
$$

Proposition 0.48. On reprend la notation ci-dessus. Le choix d'un générateur $U$ de $I \subset B$ donne lieu à un scindage $\sigma_{U}: \breve{W} \Im^{0}$ de la suite exacte $1 \longrightarrow \mathfrak{J}^{0} \longrightarrow \Im^{0} \longrightarrow \breve{W} \longrightarrow 1$ (resp. un relèvement $\left.\gamma_{U}^{0}: \mathfrak{X} \longrightarrow \Im^{0}\right)$. Un couple de générateurs $U, V$ de $I$ détermine un homomorphisme $h(U, V): \breve{W} \longrightarrow \mathfrak{J}^{0}$ tel que $: h(U, V) \sigma_{U}=\sigma_{V}$, qui est caractérisé comme l'unique homomorphisme qui vérifie

$$
h(U, V) \circ \gamma^{0}=\gamma_{V}^{0}\left(\gamma_{U}^{0}\right)^{-1} \quad \text { (cf. (0.42)). }
$$

La donnée de $h(U, V)$ équivaut à celle du système inductif $\left({ }^{(m)} h(U, V)\right)$. 
(En fait on peut établir plus généralement, grâce à la propriété universelle du couple $\left(\check{W}, \gamma^{0}\right)$, une correspondance bijective entre relèvements de $\gamma^{0}$ et les scindages de cette suite exacte).

Supposons que $A$ soit un anneau noethérien. Soit Alg.adm $\left.\right|_{A}$ la catégorie des $A$-algèbres admissibles au sens de [8]. Cette catégorie est la catégorie opposée de celle des $S$-schémas formels affines $\operatorname{Schf}$ aff $\left.\right|_{S}$.

Soit $S^{\prime}$ un $S$-schéma formel affine donné par la $A$-algèbre admissible $A^{\prime}$. Soit $K$ un idéal de définition de $S^{\prime}$. Alors $\operatorname{Spec}\left(A^{\prime} / K\right)$ est un $A$-schéma et $S^{\prime}=\lim _{\vec{K}} \operatorname{Spec}\left(A^{\prime} / K\right)$, où $K$ parcourt l'ensemble des idéaux de définition de $S^{\prime}$. On exprime ainsi $S^{\prime}$ comme une limite inductive de $S$-schémas.

DÉFInition 0.49. On définit $\hat{\Im}, \hat{\Im}^{0}, \hat{\Im}_{n}, \hat{\Im}_{n}^{0}(n \in \mathbb{N})$ comme des foncteurs en groupes de $S c h f$ aff $\left.\right|_{S}$ comme suit. Soit $S^{\prime}=\operatorname{Spf}\left(A^{\prime}\right)$. On pose alors

$$
\begin{aligned}
& \hat{\Im}\left(S^{\prime}\right)=\lim _{\overleftarrow{K}} \Im\left(A^{\prime} / K\right), \\
& \hat{\Im}^{0}\left(S^{\prime}\right)=\lim _{\overleftarrow{K}} \Im^{0}\left(A^{\prime} / K\right), \\
& \hat{\Im}_{n}\left(S^{\prime}\right)=\lim _{\overleftarrow{K}} \Im_{n}\left(A^{\prime} / K\right), \\
& \hat{\Im}_{n}^{0}\left(S^{\prime}\right)=\lim _{\overleftarrow{K}} \Im_{n}^{0}\left(A^{\prime} / K\right),
\end{aligned}
$$

où $K$ parcours l'ensemble des idéaux de définition de $A^{\prime}$.

REMARque 0.50. On peut définir $\hat{\Im}, \hat{\Im}^{0}, \hat{\Im}_{n}$, et $\hat{\Im}_{n}^{0}$ sur la catégorie des $S$-schémas formels $\left.S c h f\right|_{S}$ à partir de (0.49).

Un $S$-schéma formel $S^{\prime}$ donne lieu à un $S$-foncteur. Alors $\hat{\Im}\left(S^{\prime}\right)$ (resp. $\left.\hat{\Im}^{0}\left(S^{\prime}\right), \hat{\Im}_{n}\left(S^{\prime}\right), \hat{\Im}_{n}^{0}\left(S^{\prime}\right)\right)$ coïncide avec l'ensemble des morphismes de $S$ foncteurs de $S^{\prime}$ dans $\Im$ (resp. $\Im^{0}, \Im_{n}, \Im_{n}^{0}$ ).

Soit $U$ un générateur de $I$. On a alors un $S$-isomorphisme $\Im \simeq \check{W} \times \mathfrak{J}$ qui induit pour tout $S$-schéma formel noethérien $S^{\prime}$ un isomorphisme

$$
\hat{\Im}\left(S^{\prime}\right) \simeq \check{W}\left(S^{\prime}\right) \times \mathfrak{I}\left(S^{\prime}\right),
$$

où $\breve{W}\left(S^{\prime}\right)$ désigne les points du $S$-schéma formel $\check{W}$ à valeurs dans $S^{\prime}$. L'homomorphisme (d'augmentation) (0.25) donne lieu à un homomorphisme

$$
\varepsilon: \hat{\Im} \longrightarrow \underline{Z}_{S}
$$


de foncteurs en groupes de $\left.\operatorname{Schf}\right|_{S}$, de manière que la suite de foncteurs en groupes de Schf $\left.\right|_{S}$

$$
1 \longrightarrow \hat{\Im}^{0} \longrightarrow \hat{\Im} \longrightarrow \underline{Z}_{S} \longrightarrow 1
$$

est exacte.

Soient $A^{\prime}$ une $A$-algèbre adique (cf. [8], Déf. (7.1.9)) et $K$ un idéal de définition de $A^{\prime}$. Alors $\left(K^{n}\right)$ constitue un système fondamental de voisinages ouverts de 0 dans $A^{\prime}$. Soit $B^{\prime}=B \widehat{\otimes}_{A} A^{\prime}=B_{\left\{A^{\prime}\right\}} ;\left(K^{n} B^{\prime}\right)$ est un système fondamental d'idéaux ouverts d'une topologie linéaire de $B$.

Comme $A^{\prime}$ est noethérienne, $B^{\prime}$ est complet pour cette topologie. Cette topologie compacte coïncide avec la topologie $K B^{\prime}$-adique de $B^{\prime}$. Elle est indépendante de $K$. Soit $\widehat{\operatorname{Spec}}\left(B^{\prime}\right)$ le complété formel du $A^{\prime}$-schéma $\operatorname{Spec}\left(B^{\prime}\right)$ au-dessus de

$$
\operatorname{Spec}\left(A^{\prime} / K\right) \longrightarrow \operatorname{Spec}\left(A^{\prime}\right) .
$$

$\widehat{\operatorname{Spec}}\left(B^{\prime}\right)$ est égal au spectre formel de $B^{\prime}$ muni de la topologie $K B^{\prime}$-adique. On pose

$$
\widehat{\operatorname{omb}}\left(\mathfrak{X}^{\prime}\right)=\widehat{\operatorname{Spec}}\left(B^{\prime}\right)
$$

Noter que si $B=A[[T]]$ alors $B^{\prime}\left[I_{\left\{A^{\prime}\right\}}^{-1}\right]^{\wedge}=\lim _{\overleftarrow{n}} A^{\prime}[[T]]\left[T^{-1}\right] / K^{n} A^{\prime}[[T]]\left[T^{-1}\right]$, car $K^{n}(n \in \mathbb{N})$ est un idéal de type fini de $A^{\prime}$. Cette dernière limite s'identifie à l'anneau des séries formelles $\sum_{n=-\infty}^{+\infty} a_{n} T^{n}$ où $a_{n} \longrightarrow 0$ dans $A^{\prime}$ quand $n \longrightarrow-\infty$. D’autre part par définition on a

$$
\begin{aligned}
\Im\left(A^{\prime}\right) & =\lim _{\overleftarrow{n}}\left(A^{\prime} / K^{n}\right)[[T]]\left[T^{-1}\right]^{*}=\lim _{\overleftarrow{n}}\left(A^{\prime}[[T]]\left[T^{-1}\right] / K^{n} A^{\prime}[[T]]\left[T^{-1}\right]\right)^{*} \\
& =\left(\lim _{\overleftarrow{n}} A^{\prime}[[T]]\left[T^{-1}\right] / K^{n} A^{\prime}[[T]]\left[T^{-1}\right]\right)^{*}=\left(B^{\prime}\left[I_{\left\{A^{\prime}\right\}}^{-1}\right]^{\wedge}\right)^{*}
\end{aligned}
$$

On a finalement la

Proposition 0.52. Même hypothèse que ci-dessus sur $A^{\prime}$. Soit $B^{\prime}\left[I_{\left\{A^{\prime}\right\}}^{-1}\right]^{\wedge}$ le complété formel de $B^{\prime}\left[I_{\left\{A^{\prime}\right\}}^{-1}\right]$ par rapport à la topologie $K B^{\prime}\left[I_{\left\{A^{\prime}\right\}}^{-1}\right]$-adique. Alors que $\Im\left(A^{\prime}\right)$ s'identifie au groupe des unités $\left[B^{\prime}\left[I_{\left\{A^{\prime}\right\}}^{-1}\right]^{\wedge}\right]$, c.à.d. aux sections de $\underline{\mathbb{G}}_{m S}$ au-dessus de $\widehat{\mathfrak{U}}^{\prime}=\operatorname{Spf}\left(B^{\prime}\left[I_{\left\{A^{\prime}\right\}}^{-1}\right]^{\wedge}\right)^{\lfloor} \subset \widehat{\operatorname{omb}}\left(\mathfrak{X}^{\prime}\right)$.

Les définitions et les constructions de $\$ 1$ sont données à partir d'une courbe formelle $\mathfrak{X} \longrightarrow S$ au dessus d'une base noethérienne et affine $S$. Les 
constructions suivantes doivent permettre de définir ces même objets dans le cas où $S$ est un schéma noethérien et $\mathfrak{X} \longrightarrow S$ une courbe formelle de la forme $\mathfrak{X}=\operatorname{Spf}\left(\mathcal{O}_{S}[[T]]\right)$. On esquisse la construction d'un foncteur de la catégorie des schémas formels noethériens vers celle des espaces localement annelés

$$
X \longrightarrow \operatorname{omb}(X)
$$

qui associe à tout shcéma formel noethérien $X$ un espace localement annelé

$$
\operatorname{omb}(X)=\left(Z, \mathcal{O}_{Z}\right)
$$

noethérien, sobre (c.à.d. toute partie fermée irréductible a exactement un point générique), et avec $\mathcal{O}_{Z}$ cohérent. On a d'autre part un homomorphisme fonctoriel

$$
i_{X}: X \longrightarrow \operatorname{omb}(X)
$$

tel que $i_{X}$ est un homomorphisme de $X$ sur une partie fermée $X^{\prime}$ de omb $(X)$, de manière que l'on retrouve $X$ comme le "complété formel" de omb $(X)$ le long de $X^{\prime}$. Notons respectivement par $\operatorname{Coh}(X)$ et $\operatorname{Coh}(\operatorname{omb}(X))$ la catégorie des modules cohérents sur le schéma formel $X$ et sur l'espace annelé $\operatorname{omb}(X)$. L'on suppose l'une et l'autre munies de la structure de produit tensoriel et des isomorphismes d'associativité et de commutativité. On a alors que $i_{X}$ induit une équivalence de catégories

$$
i_{X}^{*}: \operatorname{Coh}(\operatorname{omb}(X)) \stackrel{\sim}{\longrightarrow} \operatorname{Coh}(X) .
$$

Soit $C$ un anneau adique noethérien. Soit $X=\operatorname{Spf}(C)$. On a alors un isomorphisme canonique

$$
\operatorname{omb}(X) \simeq \operatorname{Spec}(C) .
$$

Le morphisme (0.54) correspond alors au morphisme canonique $\operatorname{Spf}(C) \longrightarrow \operatorname{Spec}(C)$.

Donnons la construction de $\mathrm{omb}(X)$ pour un schéma formel noethérien $X$. Soit $P F(X)$ l'ensemble quotient de l'ensemble des idéaux cohérents $I$ de $\mathcal{O}_{X}$ par la relation d'équivalence : $« I \sim I^{\prime}$ » s'il existe un entier $n>0$ tel que $I^{n} \subset I^{\prime}$ et $I^{\prime n} \subset I^{\prime \prime}$. On a alors que $P F(X)$ avec sa relation d'ordre réticulé est l'ensemble des parties fermées d'un espace topologique sobre $\bar{X}$. Les points de $\bar{X}$ correspondent aux éléments irréductibles de l'ensemble ordonné $P F(X)$.

Soit $I$ un idéal cohérent de $\mathcal{O}_{X}$. On dénote par $V(I)$ le fermé de $\bar{X}$ défini par $I$. On définit un $\bar{X}$-faisceau en anneaux $\mathcal{O}_{\bar{X}}$ en posant

$$
\Gamma\left(\bar{X}-V(I), \mathcal{O}_{\bar{X}}\right)=\lim _{\vec{n}} \operatorname{Hom}_{\mathcal{O}_{X}}\left(I^{n}, \mathcal{O}_{X}\right) .
$$


On pose

$$
\operatorname{omb}(X)=\left(\bar{X}, \mathcal{O}_{\bar{X}}\right)
$$

On vérifie qu'étant donné un morphisme de schémas formels $f: X \longrightarrow Y$ il existe un unique morphisme d'espaces localement annelés

$$
\mathrm{omb}(f): \operatorname{omb}(X) \longrightarrow \operatorname{omb}(Y)
$$

tel que $\operatorname{omb}(f) \circ i_{X}=i_{Y} \circ f$.

Il résulte de [8], Proposition (6.9.17), pg 323, que si $C$ est un anneau adique noethérien, et si l'on pose $X=\operatorname{Spf}(C)$, la définition de $\operatorname{omb}(X)$ donne lieu à un isomorphisme canonique $\operatorname{omb}(X) \simeq \operatorname{Spec}(C)$.

Proposition 0.60. Soit $A$ un anneau noethérien. Soit $C$ une A-algèbre adique noethérienne. On pose $\mathfrak{Y}=\operatorname{Spf}(C)$ et $Y=\operatorname{Spec}(C)$.

Soit $Z$ un A-schéma séparé de présentation finie. Soit $f: \mathfrak{Y} \longrightarrow Z$ un A-morphisme formel. (On considère $Z$ comme un A-schéma formel en munissant $\mathcal{O}_{Z}$ de la topologie discrète). Il existe alors un unique $A$ morphisme

$$
\bar{f}: Y \longrightarrow Z
$$

redonnant f par restriction à $\mathfrak{Y}$, c.à.d. tel que

$$
f=\bar{f} \circ i_{\mathfrak{Y}} .
$$

PREUVE. Soitg : $\mathfrak{Y} \times_{A}$ X le $\mathfrak{Y}$-morphisme formel donné par le graphe def. Le théorème [6.4.1] de [9] montre qu'il existe un unique Y-morphisme $\bar{g}: Y \longrightarrow Y \times_{A} X$ redonnant $g$ par restriction à $\mathfrak{Y}$. Soit $\bar{f}$ le morphisme composé

$$
Y \longrightarrow Y \times_{A} X \longrightarrow X
$$

On vérifie que $\bar{f}$ satisfait aux conditions de l'énoncé.

C.Q.F.D.

\section{Jacobienne locale d'une courbe formelle relative. Énoncé du théo- rème principal}

(1.1) On donne dans ce numéro une interprétation géométrico-fonctorielle des objets introduits dans $\S 0$. On reprend la notation de $\S 0$. Soit $T$ un générateur de $I$. 
Définition 1.1.1. Soit $S^{\prime}$ un S-schéma. On pose $\mathfrak{X}^{\prime}=\mathfrak{X} \times{ }_{S} S^{\prime}$. Soit $\mathcal{O}_{\mathfrak{X}^{\prime}}\left[T^{-1}\right]$ le faisceau en $\mathcal{O}_{\mathfrak{X}^{\prime}}$-algèbres défini comme dans $\S 0$. On entend par idéal fractionnaire inversible (i.f.i) de $\mathcal{O}_{\mathfrak{x}^{\prime}}\left[T^{-1}\right]$ un $\mathcal{O}_{\mathfrak{x}^{\prime}}$-sous-module $K$ de $\mathcal{O}_{\mathfrak{X}^{\prime}}\left[T^{-1}\right]$ localement de la forme $u \mathcal{O}_{\mathfrak{X}^{\prime}}$, où $u$ désigne une section de $\mathcal{O}_{\mathfrak{X}^{\prime}}\left[T^{-1}\right]^{*}$, c.à.d. une section inversible de $\mathcal{O}_{\mathfrak{X}^{\prime}}\left[T^{-1}\right]$.

Étant données deux i.f.i. $K$ et $K^{\prime}$ de $\mathcal{O}_{x^{\prime}}\left[T^{-1}\right]$ on définit de manière évidente leur produit $K K^{\prime}=K \otimes_{\mathcal{O}_{\mathfrak{X}^{\prime}}} K^{\prime}$. On a $K=\mathcal{O}_{\mathfrak{X}^{\prime}} K=K \mathcal{O}_{\mathfrak{X}^{\prime}}$. On vérifie que l'ensemble des i.f.i. $K$ de $\mathcal{O}_{\mathfrak{X}^{\prime}}\left[T^{-1}\right]$ muni du produit ci-dessus est un groupe abélien. Un $S$-morphisme $S^{\prime \prime} \longrightarrow S^{\prime}$ induit un $S$-morphisme $f: \mathfrak{X}^{\prime \prime}=\mathfrak{X} \times_{S} S^{\prime \prime} \longrightarrow \mathfrak{X}^{\prime}$. L'image inverse $f^{*}(K)$ d'un i.f.i. $K$ de $\mathcal{O}_{\mathfrak{X}^{\prime}}\left[T^{-1}\right]$ est un i.f.i. de $\mathcal{O}_{\mathfrak{X}^{\prime \prime}}\left[T^{-1}\right]$.

DÉFINITION 1.1.2. Soit $\mathcal{P} i_{X / S}$ le $S$-foncteur en groupes abéliens faisant correspondre à $S^{\prime}$ l'ensemble des i.f.i. $K$ de $\mathcal{O}_{\mathfrak{X}^{\prime}}\left[T^{-1}\right]$.

Remarquer que l'on a un épimorphisme de faisceaux

$$
\Im \longrightarrow \mathcal{P i c} \mathfrak{x} / S
$$

envoyant une section $u$ de $\Im$ sur le i.f.i. $u \mathcal{O}_{\mathfrak{X}^{\prime}}$. On vérifie immediatement que le noyau de (1.1.3) est égal à $\mathfrak{I}^{0}$. D'autre part la suite exacte (0.11) donne lieu à la suite exacte

$$
1 \longrightarrow \mathfrak{J}^{0} \longrightarrow \Im \longrightarrow \underline{Z}_{S} \times \check{W} \longrightarrow 1,
$$

d'où l'isomorphisme

$$
\mathcal{P i c}_{\mathfrak{X} / S} \simeq \underline{Z}_{S} \times \check{W} .
$$

On a alors un homomorphisme (d'augmentation)

$$
\mathcal{P i c}_{\mathfrak{X} / S} \longrightarrow \underline{Z}_{S} .
$$

Le générateur $T$ de $I$ donne lieu par fonctorialité (cf. (0.36)) à un scindage $\sigma_{T}: \underline{Z}_{S} \longrightarrow \Im$ de la suite exacte (1.1.4). Ce qui entraîne, vu (1.1.5), que tout i.f.i. $K$ de $\mathcal{O}_{\mathfrak{X}^{\prime}}\left[T^{-1}\right]$ est globalement principal, c.à.d. de la forme $K=u \mathcal{O}_{\mathfrak{X}^{\prime}}$ avec $u \in \Gamma\left(\mathfrak{X}^{\prime}, \mathcal{O}_{\mathfrak{X}^{\prime}}\left[T^{-1}\right]\right)^{*}$.

Soit, pour tout entier $n, \mathcal{P} \mathfrak{i c}_{\mathfrak{x} / S}^{n} \subset \underline{\mathcal{P i c}}_{\mathfrak{x} / S}$ le sous-foncteur image inverse de la section de $\underline{Z}_{S}$ donnée par $n$. On a alors un isomorphisme

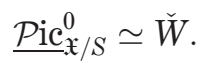


Soit $\mathfrak{X}_{n}^{\prime}=\mathfrak{X}_{n} \times_{S} S^{\prime}(n \in \mathbb{N})$. On désigne par $\underline{\mathcal{P i c}}_{\mathfrak{x}^{(n)} / S}$ le $S$-fonteur faisant correspondre à $S^{\prime}$ l'ensemble des couple $\left(K, \xi_{n}\right)$ formés d'un i.f.i. $K$ de $\mathcal{O}_{\mathfrak{X}^{\prime}}\left[T^{-1}\right]$ et d'un isomorphisme $\xi_{n}: \mathcal{O}_{\mathfrak{X}_{n}^{\prime}} \simeq K \otimes_{\mathfrak{x}_{n}^{\prime}} \mathcal{O}_{\mathfrak{X}_{n}^{\prime}}\left(=K_{n}\right)$.

Le morphisme (1.1.3) se relève en un morphisme

$$
\Im \longrightarrow \underline{\mathcal{P i c}}_{\mathfrak{x}}^{(n)} / S
$$

envoyant $u \operatorname{sur}\left(u \mathcal{O}_{\mathfrak{x}^{\prime}}, u \bmod T^{n+1}\right)$, où $u \bmod T^{n+1}$ désigne l'isomorphisme $\xi_{n}$ donné par la restriction à $\mathfrak{X}_{n}^{\prime}$ de $u$. Le noyau de (1.1.6) est celui de $\mathfrak{J}^{0} \longrightarrow \mathfrak{I}_{n}^{0}$, et ce morphisme est surjectif car $\mathfrak{J}^{0} \longrightarrow \mathfrak{J}_{n}^{0}$ l'est. On a alors un isomorphisme de $S$-foncteurs.

$$
\underline{\mathcal{P i c}}_{\mathfrak{x}^{(n)} / S} \simeq \Im_{n} \quad(\text { cf. }(0.19))
$$

La donnée de $T$ donne lieu à un isomorphisme $\Im_{n} \simeq \mathfrak{I}_{n}^{0} \times \underline{Z}_{S} \times \check{W}$. Le morphisme naturel $\underline{\mathcal{P i c}}_{\mathfrak{x}^{(n)} / S} \longrightarrow \underline{\mathcal{P i c}}_{\mathfrak{x} / S}$ définit une augmentation $\underline{\mathcal{P i c}}_{\mathfrak{x}^{(n)} / S} \longrightarrow \underline{Z}_{S}$ par la composition avec l'augmentation de $\underline{\mathcal{P i c}} \mathfrak{x} / S$.

Pour tout entier $v$, soit $\underline{\mathcal{P i c}}_{\mathfrak{x}^{(n)} / S}^{v} \subset \underline{\mathcal{P i c}}_{\mathfrak{x}^{(n)} / S}$ le sous-foncteur image inverse de la section $v$ de $\underline{Z}_{S}$ par l'augmentation $\underline{\mathcal{P i c}}_{\mathfrak{x}^{(n)} / S} \longrightarrow \underline{\mathbb{Z}}$. On a un isomorphisme

$$
\underline{\mathcal{P i c}}_{\mathfrak{x}^{(n)} / S}^{0} \simeq \Im_{n}^{0} .
$$

On pose

$$
B^{(n)}=A+I^{n+1},
$$

c.à.d. $B^{(n)}=\left\{b \in B \mid\right.$ l'image de $b-\bar{b} .1$ dans $B / I^{n+1}$ vaut zéro $\}$, où $\bar{b}$ désigne la classe de $b$ dans $B / I \simeq A$. Il est clair que $B^{(n)} \subset B$ est une $A$-sousalgèbre que l'on muni de la topologie $B^{(n)} \cap I$-adique.

Soit

$$
\mathfrak{X}^{(n)}=\operatorname{Spf}\left(B^{(n)}\right) .
$$

On a alors un $S$-morphisme de schémas formels

$$
\mathfrak{X} \longrightarrow \mathfrak{X}^{(n)}
$$

qui permet d'identifier $\mathfrak{X}^{(n)}$ à la somme amalgammée $\mathfrak{X} \coprod_{\mathfrak{x}_{n}} S$.
Posons

$$
\mathfrak{X}^{(n)^{\prime}}=\mathfrak{X}^{(n)} \times_{S} S^{\prime}
$$

et

$$
\mathcal{O}_{\mathfrak{X}^{(n)^{\prime}}}^{+}=\operatorname{Ker}\left(\mathcal{O}_{\mathfrak{X}^{(n)^{\prime}}}^{+} \longrightarrow \mathcal{O}_{S^{\prime}}^{+}\right) .
$$

Notons par $\mathcal{O}_{\mathfrak{x}}\left[T^{-1}\right]^{*} / \mathcal{O}_{\mathfrak{x}^{(n)}}^{+}$le $S$-faisceau quotient. On a alors un isomor- 
phisme de $S$-foncteurs

$$
\underline{\mathcal{P i c}}_{\mathfrak{x}^{(n)} / S} \simeq \mathcal{O}_{\mathfrak{x}}\left[T^{-1}\right]^{*} / \mathcal{O}_{\mathfrak{X}^{(n)}}^{+} .
$$

qui justifie la notation $\underline{\mathcal{P i c}} \mathfrak{x}^{(n)} / S$.

Noter que l'on a les isomorphismes suivants :

$$
\lim _{\overleftarrow{n}} \underline{\mathcal{P i c}}_{\mathfrak{x}^{(n)} / S} \simeq \Im
$$

et

$$
\lim _{\overleftarrow{n}} \underline{\mathcal{P i c}}_{\mathfrak{X}^{(n)} / S}^{0} \simeq \Im^{0}
$$

Soit $S^{\prime}=\operatorname{Spf}\left(A^{\prime}\right)$ un $S$-schéma formel affine. Si $K$ est un idéal de définition de $A^{\prime}$ on pose $S_{K}^{\prime}=\operatorname{Spec}\left(A^{\prime} / K\right)$. On a ainsi un système inductif de $S$ schémas $\left(S_{K}^{\prime}\right)$ tel que $S^{\prime}=\lim _{\vec{n}} S_{K}^{\prime}$. Posons

$$
\underline{\mathcal{P i c}} \mathfrak{x} / S\left(S^{\prime}\right)=\lim _{\overleftarrow{K}} \underline{\mathcal{P i c}} \mathfrak{x} / S\left(S_{K}^{\prime}\right)
$$

et

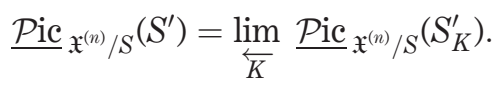

On peut interpréter l'ensemble $\underline{\mathcal{P} i c} \mathfrak{x} / S\left(S^{\prime}\right)$ (resp. $\underline{\mathcal{P i c}}_{\mathfrak{x}^{(n)} / S}\left(S^{\prime}\right)$ ) comme l'ensemble $\operatorname{Hom}_{S}\left(S^{\prime}, \underline{\mathcal{P i c}} \mathfrak{x} / S\right)\left(\operatorname{resp} \operatorname{Hom}_{S}\left(S^{\prime}, \underline{\mathcal{P i c}}_{\mathfrak{x}^{(n)} / S}\right)\right)$ de $S$-morphismes fonctoriels. On peut ainsi définir $\underline{\mathcal{P i c}} \mathfrak{x} / S$ (resp. $\underline{\mathcal{P i c}} \mathfrak{x}^{(n)} / S$ ) comme un foncteur de $\left.\mathrm{Schf}\right|_{S}$. L'isomorphisme (1.1.7) donne lieu à un isomorphisme de foncteurs de $\left.\operatorname{Schf}\right|_{S}$

$$
\underline{\mathcal{P i c}}_{\mathfrak{x}^{(n)} / S} \simeq \hat{\Im}_{n}\left(\simeq \mathfrak{J}_{n} \times \check{W}(\operatorname{cf} .(0.17))\right) .
$$

REMARQue 1.1.18. Soient $\mathfrak{X}^{\prime}=\mathfrak{X} \times_{S} \mathfrak{X}$ et $\mathfrak{I}_{\Delta} \subset \mathcal{O}_{\mathfrak{x}}^{\prime}$ l'idéal de définition de la diagonale $\Delta \subset \mathfrak{X} \times_{S} \mathfrak{X}$. Notons par $\mathfrak{I}_{\Delta m}$ l'idéal de $\mathcal{O}_{\mathfrak{X}_{\times_{S}} \mathfrak{x}_{m}}$ induit par $\mathfrak{I}_{\Delta}$. On a alors que $\mathfrak{I}_{\Delta m}$ est un i.f.i. de $\mathcal{O}_{\mathfrak{x} \times \times_{S} \mathfrak{x}_{m}}\left[T^{-1}\right]$, et le système projectif $\left(\mathfrak{I}_{\Delta m}\right)$ détermine une section de $\underline{\mathcal{P} i c} \mathfrak{x} / S$ au-dessus de $\mathfrak{X}$, qui correspond à la section $\gamma^{1}$ (cf. (0.35), (iii)) par l'isomorphisme (1.1.5).

Soient un entier $m>0$, et un entier $1<i \leq m+1$. Posons $\mathfrak{Y}=\prod^{m+1} \mathfrak{X}$. Notons par

$$
p: \prod^{m+1} \mathfrak{X} \longrightarrow \mathfrak{X}
$$


la première projection, et pour tout $1<i \leq m+1$ :

$$
p_{i}: \prod^{m+1} \mathfrak{X} \longrightarrow \mathfrak{X} \times_{S} \mathfrak{X}
$$

le morphisme défini par la première et la $i$-ème projection. Soit $\mathfrak{I} \subset \mathcal{O}_{\mathfrak{X}}$ l'idéal donné par $I \subset B$. On vérifie que le $\mathcal{O}_{\mathfrak{Y}}$-module inversible

$$
p_{1}^{*}\left(\mathfrak{I}_{4}\right) \otimes_{\mathcal{O}_{\mathfrak{Y}}} \ldots \otimes_{\mathcal{O}_{\mathfrak{Y}}} p_{m}^{*}\left(\mathfrak{J}_{4}\right)
$$

provient d'un $\mathcal{O}_{\mathfrak{x}} \times_{S} W_{m}$-module inversible $\operatorname{Sym}^{(m)}\left(\mathfrak{I}_{4}\right)$ par l'image inverse par le morphisme canonique :

$$
\mathfrak{Y}=\prod^{m+1} \mathfrak{X} \longrightarrow \mathfrak{X} \times{ }_{S} \operatorname{Sym}^{(m)}(\mathfrak{X}) .
$$

Soit

$$
\operatorname{Sym}^{(m)}\left(\mathfrak{J}_{4}\right)^{\prime}=p^{*}\left(\mathfrak{I}^{\otimes-m} \otimes_{\mathcal{O}_{\mathfrak{Y}}} \operatorname{Sym}^{(m)}\left(\mathfrak{J}_{4}\right) .\right.
$$

Alors $\operatorname{Sym}^{(m)}\left(\mathfrak{J}_{4}\right)^{\prime}$ est un i.f.i. de $\mathcal{O}_{\mathfrak{x} \times{ }_{S} W_{m}}\left[T^{-1}\right]$, et défini une section de $\underline{\mathcal{P i c}} \mathfrak{x}_{\mathfrak{x} / S}^{0}$ au-dessus de $W_{m}$, et qui coïncide avec $\operatorname{Sym}^{(m)}\left(\gamma^{0}\right)$.

Soit $\underline{y}$ une section de $\underline{Z}_{S}$. On définit un i.f.i. de $\mathcal{O}_{\mathfrak{X} \times{ }_{S} W_{m}}\left[T^{-1}\right]$ en posant

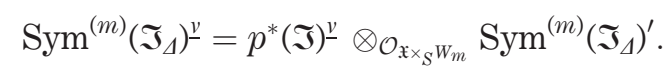

Soit $\left(\operatorname{Sym}^{(m)}\left(\Im_{4}\right)^{\underline{\nu}}\right)^{*}$ le fibré principal associé. On désigne par $\operatorname{Sym}^{(m)}\left(\mathfrak{I}_{4}\right)_{n}^{\frac{v}{n}}$ sa restriction à $\mathfrak{X}_{n} \times W_{m}$ comme module inversible. Soit $\left(\operatorname{Sym}^{(m)}\left(\Im_{4}\right)_{n}^{\frac{v}{n}}\right)^{*}$ le fibré principal associé. Notons par pr (resp. pr ${ }^{n}$ ) la projection de $\mathfrak{X} \times_{S} W_{m}$ (resp. $\left.\mathfrak{X}_{n} \times_{S} W_{m}\right)$ sur $W_{m}$. Alors le faisceau image directe

$$
\begin{gathered}
\operatorname{pr}_{*}\left(\left(\operatorname{Sym}^{(m)}\left(\mathfrak{J}_{4}\right)^{\frac{v}{}}\right)^{*}\right) \\
\left(\operatorname{resp} \cdot \operatorname{pr}_{*}^{n}\left(\left(\operatorname{Sym}^{(m)}\left(\mathfrak{J}_{4}\right)^{\frac{v}{n}}\right)^{*}\right)\right)
\end{gathered}
$$

est un $\underline{\mathrm{G}}_{m}(\mathfrak{X})$-torseur (resp. $\underline{\mathrm{G}}_{m}\left(\mathfrak{X}_{n}\right)$-torseur) au-dessus de $W_{m}$ qui s’identifie canoniquement $\grave{\mathbf{a}}^{(m)} \Im^{\underline{\underline{\nu}}}$ (resp. $\left.{ }^{(m)} \Im_{n}^{\underline{\nu}}\right)$.

Soit $S \longrightarrow \underline{Z}_{S} \times_{S} \check{W}$ une section admettant une factorisation à travers $S \longrightarrow \underline{Z}_{S} \times{ }_{S} W_{m}$.

Soit $\underline{v}$ la section de $\underline{Z}_{S}$ donnée par $S \longrightarrow \underline{Z}_{S} \times \check{W}$. Alors il lui correspond le i.f.i. de $\mathcal{O}_{\mathfrak{X}_{S}}\left[T^{-1}\right]$ l'image inverse de $\operatorname{Sym}^{(m)}\left(\mathfrak{I}_{4}\right)^{\underline{v}}$ par le morphisme induit $\mathfrak{X}_{S} \longrightarrow \mathfrak{X}_{W_{m}}$.

On suppose maintenant $A$ noethérien. Soit $A^{\prime}$ une $A$-algèbre adique. Soit $K$ un idéal de définition de $A^{\prime}$. Posons $S^{\prime}=\operatorname{Spf}\left(A^{\prime}\right)$ et $S_{m}^{\prime}=\operatorname{Spf}\left(A^{\prime} / K^{m}\right)(m \in \mathbb{N})$. Alors $B^{\prime}=B \widehat{\otimes}_{A} A^{\prime}$ est une $A^{\prime}$-algèbre com- 
plète pour la topologie $K B^{\prime}$-adique de manière que le spectre formel de $B^{\prime}$ muni de cette topologie, s'identifie au complété formel $\widehat{\operatorname{omb}}\left(\mathfrak{X}^{\prime}\right)$, où $\mathfrak{X}^{\prime}=\operatorname{Spf}\left(B^{\prime}\right)$, le long de $S_{1}^{\prime}=\operatorname{Spec}\left(A^{\prime} / K\right)$, comme il résulte du fait que $A^{\prime}$ est noethérien. On a alors

$$
\operatorname{omb}\left(\mathfrak{X} \times_{S} S_{m}^{\prime}\right) \simeq \operatorname{omb}\left(\mathfrak{X}^{\prime}\right) \times_{S^{\prime}} S_{m}^{\prime} .
$$

Une section de $\underline{\mathcal{P i c}} \underset{\mathfrak{O} / S}{ }$ au-dessus de $S_{m}^{\prime}$ correspond à la donnée d'un idéal principal $L_{m} \subset \overline{\mathcal{O}}_{\mathfrak{x} \times{ }_{S} S_{m}^{\prime}}\left[T^{-1}\right]$ lequel donne lieu à un idéal inversible de $\operatorname{omb}\left(\mathfrak{X} \times_{S} S_{m}^{\prime}\right)$. D’où la donnée d'une section de

$$
\underline{\mathcal{P i c}} \mathfrak{x} / S\left(S^{\prime}\right)=\lim _{\overleftarrow{m}} \underline{\mathcal{P i c}} \mathfrak{x} / S\left(S_{m}^{\prime}\right)
$$

entraîne celle d'une famille $\left(L_{m}\right)$ de modules inversibles au-dessus du système inductif $\left(\operatorname{omb}\left(\mathfrak{X}^{\prime}\right) \times_{S^{\prime}} S_{m}^{\prime}\right)$, et à fortiori celle d'un $\mathcal{O}_{\widehat{o m b}\left(\mathfrak{X}^{\prime}\right)}$-module inversible $L$. Soit $\widehat{\mathfrak{U}}^{\prime} \subset \widehat{\mathrm{omb}}\left(\mathfrak{X}^{\prime}\right)$ l'ouvert défini comme dans $(0.52)$. On vérifie qu'il existe une section

$$
u \in \Gamma\left(\widehat{\mathfrak{U}}^{\prime}, \mathcal{O}_{\widehat{\operatorname{omb}}\left(\mathfrak{x}^{\prime}\right)}^{*}\right)
$$

telle que

$$
K=u \mathcal{O}_{\widehat{\operatorname{omb}\left(\mathfrak{x}^{\prime}\right)}}^{*} \subset \mathcal{O}_{\widehat{\mathfrak{l}^{\prime}}}^{*}
$$

Lemme 1.1.19. Avec les notations ci-dessus, la donnée d'une section de $\underline{\mathcal{P} i c}_{\mathfrak{X}^{(n)} / S}$ au-dessus de $S^{\prime}$, équivaut à celle d'un couple $\left(L, \xi_{n}\right)$ formé d'un $\mathcal{O}_{\widehat{o m b}\left(\mathfrak{x}^{\prime}\right)}$-module inversible $L$ de la forme

$$
L=u \mathcal{O}_{\widehat{o m b}\left(\mathfrak{x}^{\prime}\right)}, u \in \Gamma\left(\widehat{\mathfrak{U}}^{\prime}, \mathcal{O}_{\widehat{\operatorname{omb}}\left(\mathfrak{x}^{\prime}\right)}^{*}\right)
$$

et d'un isomorphisme

$$
\xi_{n}: \mathcal{O}_{\mathfrak{X}_{n}^{\prime}} \simeq L \otimes_{\mathcal{O}_{\mathfrak{X}^{\prime}}} \mathcal{O}_{\mathfrak{X}_{n}^{\prime}}(\text { cf. }(0.61)) .
$$

(1.2). Soit $S$ un schéma. Soit $X \longrightarrow S$ une $S$-courbe projective et lisse muni d'un diviseur de Cartier $D \longrightarrow X$ relatif effectif. Notons par $p^{(n)}: D^{(n)} \longrightarrow S$ le $n$-ème voisinage infinitésimal de $D$ dans $X$. Soit

$$
X^{(n)}=X \coprod_{D^{(n)}} S
$$

la somme amalgammée de $X$ avec $S$ le long de $D^{(n)}$ (cf. [3], [5]). 
Posons pour tout entier $n>0$

$$
J_{n}=\underline{\operatorname{Pic}}_{X^{(n)} / S}\left(\text { resp. } J=\lim _{\overleftarrow{n}} \underline{\operatorname{Pic}}_{X^{(n)} / S}\right)
$$

(cf. loc. cit.). Le $S$-schéma $X^{(n)}$ admet une section canonique

$$
S \longrightarrow X^{(n)}=X \coprod_{D^{(n)}} S
$$

et l'on peut donc décrire les sections de $J_{n}$ au-dessus du $S$-schéma $S^{\prime}$ comme étant l'ensemble des classes d'isomorphisme des couples $\left(L, \xi_{n}\right)$ formés d'un $\mathcal{O}_{X_{S^{\prime}}}$-module inversible $L$ et d'une trivialisation

$$
\xi_{n}: \mathcal{O}_{D_{S^{\prime}}^{(n)}} \simeq L \otimes_{\mathcal{O}_{X_{S^{\prime}}}} \mathcal{O}_{D_{S^{\prime}}^{(n)}}
$$

Soit $\Delta^{\prime} \subset X \times_{S}(X-D)$ le diviseur de Cartier donné par l'image de la restriction à $X-D$ du morphisme diagonal

$$
\Delta: X \longrightarrow X \times X \text {. }
$$

On désigne par $\Phi^{(n)}$ la section de $J_{n}$ au-dessus de $X-D$, donnée par la classe d'isomorphisme du couple $\left(L_{\Delta^{\prime}}, \underline{1}\right)$ formé de l'idéal de définition $L_{\Delta^{\prime}}$, et de la trivialisation de $L_{A^{\prime}}$ le long de $D^{(n)} \times_{S}(X-D)$ donnée par la section 1 de $L_{\Lambda^{\prime}}$. D'après loc. cit., on a pour tout $S$-schéma en groupes commutatif et lisse $G$, une bijection

$$
\operatorname{Hom}_{S-g r}(J, G) \simeq G(X-D)
$$
induite par la composition avec la section $\Phi=\lim _{\overleftarrow{n}} \Phi^{(n)}$ de $J$ au-dessus de
$X-D$.

Pour tout entier $n \geq 0$ on a une suite exacte de $S$-faisceaux fpqc en groupes

$$
1 \longrightarrow \underline{\mathrm{G}}_{\mathrm{mS}} \longrightarrow p_{*}^{(n)}\left(\underline{\mathrm{G}}_{m} D^{(n)}\right) \longrightarrow J_{n} \longrightarrow \underline{\mathrm{Pic}}_{X / S} \longrightarrow 1
$$

Supposons que le diviseur $D$ soit une réunion disjointe :

$$
D=\coprod_{i=1}^{v} D_{i}
$$

où $D_{i}$ désigne l'image d'une section $S \longrightarrow X$. Soit $\mathfrak{X}_{1}$ la $S$-courbe formelle affine obtenue par la complétion formelle de $X$ le long de $D_{i}$. On a alors

$$
\mathfrak{x}_{i}=\lim _{\vec{n}} D_{i}^{(n)} \text {. }
$$


On pose $\mathfrak{X}_{i n}=D_{i}^{(n)}$. On définit $\underline{\mathrm{G}}_{\mathrm{mS}}\left(\mathfrak{X}_{i}\right)\left(\operatorname{resp} \cdot \underline{\mathrm{G}}_{\mathrm{mS}}\left(\mathfrak{X}_{i n}\right)\right)$ comme dans $\S 0$ avec $\mathfrak{X}=\mathfrak{X}_{i}$. On a alors un isomorphisme de $S$-groupes :

$$
\prod_{i=1}^{v} \underline{\mathrm{G}}_{\mathrm{mS}}\left(\mathfrak{x}_{i n}\right) \simeq p_{*}^{(n)}\left(\underline{\mathrm{G}}_{m} D^{(n)}\right) .
$$

Supposons maintenant que $S=\operatorname{Spec}(A)$, où $A$ désigne un anneau noethérien, et que pour tout $i=1, \ldots, v$ on ait un isomorphisme

$$
\mathfrak{X}_{i} \simeq \operatorname{Spf}(A[[T]]) .
$$

Un tel isomorphisme existe toujours localement sur $S$. On pose $B_{i}=\Gamma\left(\mathfrak{X}_{i}, \mathcal{O}_{\mathfrak{x}_{i}}\right)$. On a alors que $B_{i}$ est une $A$-algèbre adique augmentée isomorphe à $A[[T]]$. Notons par $L_{D_{i}}$ l'idéal de définition de $D_{i}$ dans $\mathcal{O}_{X}$. Soit $I_{i} \subset B_{i}$ l'idéal correspondant à $L_{D_{i}} \otimes_{\mathcal{O}_{X}} \mathcal{O}_{\mathfrak{x}_{i}}$. On a alors un isomorphisme

$$
D_{i} \simeq \operatorname{Spec}\left(B_{i} / I_{i}\right)\left(\operatorname{resp.} D_{i}^{(n)}=\mathfrak{X}_{i n}=\operatorname{Spec}\left(B_{i} / I_{i}^{n+1}\right)\right),
$$

c.à.d. l'espace sous-jacent à $\mathfrak{X}_{i}\left(\right.$ resp. $\left.\mathfrak{X}_{i n}\right)$ s'identifie à $D_{i}$.

On pose

$$
\mathfrak{U}_{i}=\operatorname{Spec}\left(B_{i}\left[I_{i}^{-1}\right]\right)=\operatorname{omb}\left(\mathfrak{X}_{i}\right)-D_{i} .
$$

et (comme dans $\S 0$ )

$$
{ }^{i} \Im=\underline{\mathrm{G}}_{\mathrm{mS}}\left(\mathfrak{U}_{i}\right) \quad\left(\operatorname{resp} \cdot{ }^{i} \mathfrak{J}^{0}=\underline{\mathrm{G}}_{m S}\left(\mathfrak{X}_{i}\right),{ }^{i} \mathfrak{J}_{n}^{0}=\underline{\mathrm{G}}_{\mathrm{mS}}\left(\mathfrak{X}_{i n}\right)\right),
$$

et l'on définit ${ }^{i} \mathfrak{J} \subset{ }^{i} \Im$ comme le $S$-groupe engendré $\operatorname{par}^{i} \mathfrak{J}^{0}$ et la section de ${ }^{i} \Im$ donnée par un générateur $T_{i}$ de $I_{i}$, et pour tout entier $n \geq 0,{ }^{i} \Im_{n}$ (resp. ${ }^{i} \Im_{n}$ ) comme dans (0.17) (resp. (0.19)).

On donne, dans ce paragraphe, quelques rapports existants entre ${ }^{i} \Im,{ }^{i} \mathfrak{J}$ et $J$. Pour tout $S$-schéma $S^{\prime}$ on pose $X^{\prime}=X \times_{S} S^{\prime}, D_{i} \times_{S} S^{\prime}$, et $L_{D_{i}^{\prime}}=L_{D_{i}} \otimes_{\mathcal{O}_{S}} \mathcal{O}_{S^{\prime}}$. Soit

$$
{ }^{i} \mathcal{O}_{X^{\prime}(n)}^{+}=\operatorname{Ker}\left(\mathcal{O}_{X^{\prime}}^{*} \longrightarrow\left(\mathcal{O}_{X^{\prime}} / L_{D_{i}^{\prime}}^{n+1}\right)^{*}\right) .
$$

L'immersion fermée $j_{i}: D_{i} \longrightarrow X$ en induit une autre $j_{i}^{\prime}: D_{i}^{\prime} \longrightarrow X^{\prime}$. Soit $\mathcal{O}_{X^{\prime}}\left[L_{D_{i}}^{-1}\right]$ le faisceau d'anneaux de fractions à dénominateurs dans le sousfaisceau des générateurs de $L_{D_{i}^{\prime}}$. Soit $i \widetilde{\Im}_{n}$ le $S$-faisceau de Zariski défini par :

$$
S^{\prime} \longrightarrow \Gamma\left(D_{i}^{\prime}, j_{i}^{\prime}-1\left(\mathcal{O}_{X^{\prime}}\left[L_{D_{i}^{\prime}}^{-1}\right]^{*} /{ }^{i} \mathcal{O}_{\widehat{X}^{\prime}(n)}^{+}\right)\right) .
$$


Il est clair que l'on a une bijection :

$$
i \bar{\Im}_{n}\left(S^{\prime}\right) \stackrel{\sim}{\longrightarrow} \Gamma\left(X^{\prime}, \mathcal{O}_{X^{\prime}}\left[L_{D_{i}^{\prime}}^{-1}\right]^{*} /{ }^{i} \mathcal{O}_{\widehat{X}^{\prime}(n)}^{+}\right),
$$

car le support de $\mathcal{O}_{X^{\prime}}^{*}\left[L_{D_{i}}^{-1}\right]^{*} /{ }^{i} \mathcal{O}_{X^{\prime}(n)}^{+}$est $D_{i}^{\prime}$. Une section $\bar{u}$ de ce faisceau audessus de $X^{\prime}$ donne lieu à un $\mathcal{O}_{X^{\prime}}$-sous-module inversible $K$ de $\mathcal{O}_{X^{\prime}}\left[L_{D_{i}}^{-1}\right]$, localement engendré par un représentant $u$ de $\bar{u}$, muni d'un isomorphisme :

$$
\xi_{n}: \mathcal{O}_{\mathfrak{X}_{i n}^{\prime}} \simeq K \otimes_{\mathcal{O}_{X^{\prime}}} \mathcal{O}_{\mathfrak{x}_{i n}^{\prime}}\left(\mathfrak{X}_{i n}^{\prime}=\mathfrak{X}_{i n} \times_{S} S^{\prime}\right) \text {. }
$$

(Remarquer que pour tout $k=1, \ldots, v, k \neq i$, on a que $K \otimes_{\mathcal{O}_{X^{\prime}}} \mathcal{O}_{\mathfrak{X}_{k n}^{\prime}}$ est canoniquement isomorphe à $\left.\mathcal{O}_{\mathfrak{X}_{k n}^{\prime}}\right)$. En faisant correspondre à $\bar{u}$ la section de $J_{n}$ au-dessus de $S^{\prime}$ donnée par le couple $\left(K, \xi_{n}\right)$ on obtient un morphisme de $S$-groupes

$$
j_{i}^{-1}\left(\mathcal{O}_{X}\left[L_{D_{i}}^{-1}\right]^{*} /{ }^{i} \mathcal{O}_{X^{(n)}}^{+}\right) \longrightarrow J_{n} .
$$

D'autre part le morphisme $\mathfrak{X}_{i n} \longrightarrow X$ donne lieu à un morphisme de $S$ groupes

$$
i \bar{\Im}_{n} \longrightarrow \mathcal{O}_{\mathfrak{x}_{i}}\left[T^{-1}\right]^{*} / \mathcal{O}_{\mathfrak{x}_{i n}}^{+} \simeq \underline{\mathcal{P i c}}_{\mathfrak{x}_{i} / S}^{(n)}
$$

où $T$ est un générateur de $I_{i}$. Le lemme (0.8) montre que (1.2.7) est en fait un isomorphisme.

À partir du morphisme (1.2.6) et de l'isomorphisme (1.2.7) on définit un homomorphisme de $S$-groupes

$$
i_{\Im_{n}} \simeq \underline{\mathrm{Pic}}_{\mathfrak{x}_{i}^{(n)}} / S \longrightarrow J_{n},
$$

et on a un diagramme commutatif

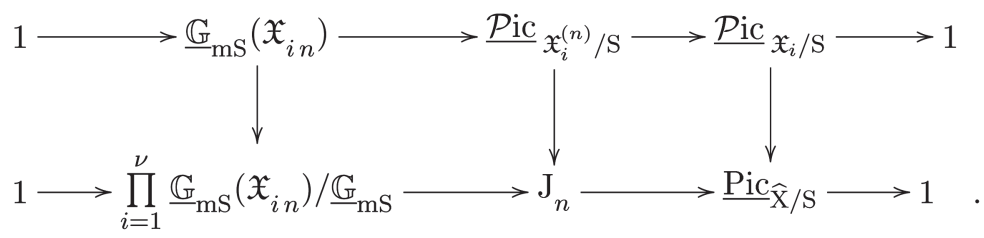

Notons par

$$
\varepsilon_{i}:{ }^{i} \Im \longrightarrow \underline{Z}_{S}\left(\operatorname{resp} . \varepsilon_{i m}:{ }^{i} \Im_{m} \longrightarrow \underline{Z}_{S}\right)
$$

le $S$-homomorphisme d'augmentation défini comme dans (0.25) (resp. $(0.26))$. 
Soit $\varepsilon_{i m}^{\prime}$ (resp. $\varepsilon_{i}^{\prime}$ ) la restriction de $\varepsilon_{i m}$ (resp. $\left.\varepsilon_{i}\right) \grave{a}^{i} \mathfrak{J}_{m}$ (resp. $\left.{ }^{i} \mathfrak{J}\right)$.

Noter que $\varepsilon_{i m}$ (resp. $\varepsilon_{i}$ ) est compatible avec l'augmentation canonique

$$
\underline{\mathrm{Pic}}_{\widehat{X}^{(m)} / S} \longrightarrow \underline{\mathbb{Z}}_{S}\left(\operatorname{resp} . \lim _{\overleftarrow{m}} \underline{\mathrm{Pic}}_{\widehat{X}^{(m)} / S} \longrightarrow \underline{Z}_{S}\right) .
$$

Le morphisme (1.2.8) donne lieu par restriction à un morphisme

$$
\mathfrak{I}_{n} \longrightarrow J_{n}
$$

et finalement à un morphisme

$$
\prod_{i=1}^{v} i \mathfrak{J}_{n} \longrightarrow J_{n}
$$

Par passage à la limite en $n$ on obtient un morphisme de $S$-groupes

$$
\prod_{i=1}^{v} i \mathfrak{J} \longrightarrow \lim _{\overleftarrow{n}} J_{n}
$$

Soit $T_{i}$ un générateur de $I_{i}$. Soit $\varphi_{T_{i}}^{1} \in \mathfrak{J}\left(\mathfrak{U}_{i}\right)$ la section définie à partir de $T_{i}$ comme dans (0.31), (iii). Notons par $\Phi_{T_{i}}^{1}$ l'image de $\varphi_{T_{i}}^{1}$ par le $S$-homomorphisme ${ }^{i \mathfrak{J}} \longrightarrow J$ induit par (1.2.12). Soit $\Phi_{\mathfrak{U}_{i}}$ la fibre de $\Phi$ en $\mathfrak{U}_{i} \longrightarrow X-D$. Le lemme suivant exprime la compatibilité de la propriété universelle de la jacobienne globale $(J, \Phi)$ de $X-D$ avec la propriété universelle de $\left({ }^{i} \mathfrak{J}, \varphi_{T_{i}}^{1}\right)$ (cf. Théorème (1.4.4)).

LEMme 1.2.13. Avec les notations ci-dessus on $a$ :

(i) $-\Phi_{T_{i}}^{1} \equiv \Phi_{\mathfrak{U}_{i}}\left(\bmod \operatorname{Ker}\left(J\left(\operatorname{omb}\left(\mathfrak{X}_{i}\right)\right) \longrightarrow J(S)\right)\right)$;

(ii) - Pour tout S-schéma en groupes commutatif et lisse G le diagramme suivant est commutatif

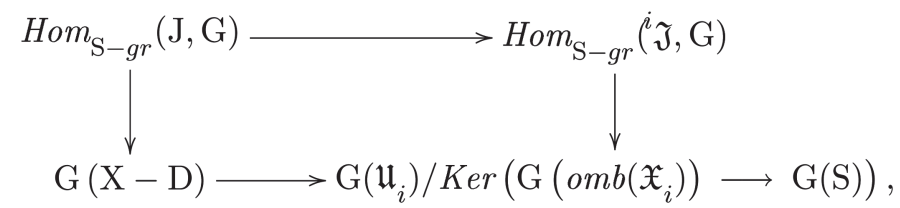

où la première (resp. deuxième) flèche verticale est induite par $\Phi$ (resp. $\left.\varphi_{T_{i}}^{1}\right)$, la flèche horizontale supérieure (resp. inférieure) est induite par (1.2.12) (resp. la restriction $G(X-D) \longrightarrow G\left(\mathfrak{U}_{i}\right)$ ). 
Preuve. Le (ii) résulte immédiatement de (i), montrons donc (i). Posons pour simplifier la notation $D=D_{i}, \mathfrak{J}={ }^{i} \mathfrak{I}, \mathfrak{X}=\mathfrak{X}_{1}, \mathfrak{U}=\mathfrak{U}_{1}$ et $v=1$. On a alors $D^{(n)}=\mathfrak{X}_{n}$ et $\mathfrak{X}=\lim _{\vec{n}} D^{(n)}=\lim _{\vec{n}} \mathfrak{X}_{n}$. Notons par $L_{\Delta}$ l'idéal de définition de la diagonale $\Delta \subset X^{\prime}=X \times_{S} X$, et par $L_{\Delta^{\prime}}$ la restriction de $L_{\Delta} \grave{a} X \times_{S}(X-D)$. Par définition $\Phi$ est la section de $J$ au-dessus de $X-D$ donnée par le couple $\left(L_{\Delta^{\prime}}, \underline{1}\right)$, où

$$
\underline{1} \in \Gamma\left(\mathfrak{X} \times_{S}(X-D), L_{\Delta^{\prime}} \otimes_{\mathcal{O}_{X_{X_{S}}(X-D)}} \mathcal{O}_{\mathfrak{X} \times_{S}(X-D)}\right) .
$$

Notons par $\left(L_{\Delta_{\mathfrak{l}}}, 1_{\mathfrak{u}}\right)$ le couple formé par le $\mathcal{O}_{X_{\mathfrak{U}}}$-module inversible $L_{\Delta_{\mathfrak{U}}}$ obtenu à partir de $L_{\Delta}$ par passage à la fibre $X_{\mathfrak{U}}=X \times_{S} \mathfrak{U} \subset X \times_{S}(X-D)$, et par la trivialisation $\underline{1}_{\mathfrak{u}}$ de $L_{\Delta_{\mathfrak{l}}}$ le long de $\mathfrak{X} \times_{S} \mathfrak{U}$, induite par celle de $L_{\Delta^{\prime}}$ le long de $\mathfrak{X} \times_{S}(X-D)$. Il est clair que la fibre $\Phi_{\mathfrak{U}}$ de $\Phi$ est donnée par la classe de $\left(L_{\Delta_{\mathfrak{H}}}, \underline{1}_{\mathfrak{H}}\right)$.

Quitte à substituer S par un recouvrement de Zariski $S^{\prime} \longrightarrow S$ on peut supposer :

(1) qu'il existe une fonction méromorphe relative $T$ de $X$ et un voisinage ouvert $U$ de $D$, contenu dans le domaine de définition de $T$, tel que:

$$
L_{D} \mid U=T \cdot \mathcal{O}_{U}
$$

c.à.d. $T$ donne lieu par restriction à $U$, à un générateur de $L_{D} \mid U$.

(2) Posons $X_{U}=X \times_{S} U, \Delta_{U}=\Delta \cap X_{U}$, et $D_{U}=D \times_{S} U$. Notons toujours par $T$ la fonction méromorphe relative de $X \times_{S} U$ donnée par $T \otimes 1$. Soit $t \in \Gamma\left(X_{U}, \mathcal{O}_{X_{U}}\right)$ la section donnée par $1 \otimes T$. Il existe un voisinage ouvert $W \subset U \times_{S} U$ de $D_{U}$ tel que :

$$
L_{\Delta} \mid W=(t-T) \cdot \mathcal{O}_{W}
$$

On a alors $L_{D_{U}} \mid W=T . \mathcal{O}_{W}$.

On définit une fonction méromorphe relative de $X_{U}$ en posant:

$$
m=T(t-T)^{-1} .
$$

Notons par $m\left(L_{\Delta_{U}}\right)$ le $\mathcal{O}_{X_{U}}$-module inversible fractionnaire de $X_{U}$ défini par :

$$
\Gamma\left(V, m\left(L_{\Delta_{U}}\right)\right)=\left\{m s \mid s \in \Gamma\left(V, L_{\Delta_{U}}\right)\right\},
$$

où $L_{\Delta_{U}}$ désigne l'idéal de définition de $\Delta_{U}$ dans $X_{U}$.

On a ainsi un isomorphisme :

$$
m: L_{D_{U}} \stackrel{\sim}{\longrightarrow} m\left(L_{\Delta_{U}}\right)
$$


donné par la multiplication par m. Posons

$$
L=L_{D_{U}}^{-1} \otimes_{\mathcal{O}_{X_{U}}} m\left(L_{\Delta_{U}}\right) .
$$

Il résulte de (2) et de la définition de $m$ (resp. de l'isomorphisme (*)) que :

$$
L \mid W=\mathcal{O}_{W}
$$

et que la fibre $(L)_{X}$ de $L$ en $X=X \times_{S} D \longrightarrow X_{U}$ est égale à $\mathcal{O}_{X}$. Il est alors clair que le $\mathcal{O}_{X_{U}}$-module inversible L est muni de la trivialisation évidente $\underline{1}$ au-dessus de $W$. Soit $\left(L_{\mathfrak{U}}, \underline{1}_{\mathfrak{U}}\right)$ le couple formé du module donné par la fibre de $L$ en $\mathfrak{U} \longrightarrow U$, et de la trivialisation de $L_{\mathfrak{U}}$ le long de $D \times_{S} \mathfrak{U}$ induite par 1 . Soit $m_{\mathfrak{U}}$ la fibre de l'isomorphisme $(*)$ en $\mathfrak{U}$. On a alors un isomorphisme induit par $m_{\mathfrak{l}}$ :

$$
\left(L_{\Delta_{\mathfrak{l}}}, \underline{1}_{\mathfrak{l}}\right) \simeq\left(L_{D_{\mathfrak{l}}}, \underline{m}_{\mathfrak{l}}(\underline{1})\right)\left(L_{\mathfrak{U}}, \underline{1}_{\mathfrak{l}}\right),
$$

où $L_{D_{\mathfrak{l}}}$ désigne la fibre de $L_{D}$ en $\mathfrak{U}$ (= idéal de définition de $D_{U}$ dans $\left.X \times_{S} \mathfrak{U}\right)$.

Notons que la classe de $\left(L_{D_{\mathfrak{I}^{\prime}}}, \underline{m}_{\mathfrak{U}}(1)\right)$ donne une section $J$ au-dessus de $\mathfrak{U}$ qui cö̈ncide avec l'image de $\varphi_{T}^{1}$ par (1.2.12) (Noter aussi que la fonction méromorphe $T$ donne un générateur de $I \subset B)$, où $\varphi_{T}^{1}$ est défini d'après (0.31), (iii). D'autre part on a que la classe de $\left(L_{\mathfrak{U}}, \underline{1}_{\mathfrak{U}}\right)$ donne lieu, d'après ce qui précède, à une section de

$$
\operatorname{Ker}(J(o m b(\mathfrak{X})) \longrightarrow J(S)) .
$$

L'affirmation (i) résulte alors de l'isomorphisme (**).

C.Q.F.D.

(1.3) Soit

$$
X=\mathbb{P}_{S}^{1}=\operatorname{Proj}\left(A\left[T_{0}, T_{1}\right]\right)(S-\text { droite projective }) .
$$

On pose

$$
D_{(0)}=V\left(T_{1}\right) \quad\left(\operatorname{resp} . D_{(\infty)}=V\left(T_{0}\right)\right)
$$

et

$$
D=D_{(0)} \coprod D_{(\infty)}
$$

Soit

$$
U_{(\infty)}=\mathbb{P}_{S}^{1}-D_{(0)}=\operatorname{Spec}\left(A\left[T_{0}, T_{1}\right]_{\left(T_{1}\right)}\right)
$$

(resp. $U_{(0)}=\mathbb{P}_{S}^{1}-D_{(\infty)}=\operatorname{Spec}\left(A\left[T_{0}, T_{1}\right]_{\left(T_{0}\right)}\right)$,

$$
\left.U=\mathbb{P}_{S}^{1}-D=\operatorname{Spec}\left(A\left[T_{0}, T_{1}\right]_{\left(T_{0}, T_{1}\right)}\right)\right) .
$$


Noter que $U_{(0)}, U_{(\infty)}$ et $U$ sont des ouverts affines de $\mathbb{P}_{S}^{1}$. Soit $\mathfrak{X}_{(0)}$ (resp. $\left.\mathfrak{X}_{(\infty)}\right)$ le complété formel de $X$ le long de $D_{(0)}$ (resp. $\left.D_{(\infty)}\right)$. On pose

$$
B_{(0)}=\Gamma\left(\mathfrak{X}_{(0)}, \mathcal{O}_{\mathfrak{X}_{(0)}}\right) \quad\left(\operatorname{resp} . B_{(\infty)}=\Gamma\left(\mathfrak{X}_{(\infty)}, \mathcal{O}_{\mathfrak{x}_{(\infty)}}\right)\right),
$$

et

$$
\left.I_{(0)}=\operatorname{Ker}\left(B_{(0)} \longrightarrow A\right) \text { (resp. } I_{(\infty)}=\operatorname{Ker}\left(B_{(\infty)} \longrightarrow A\right)\right)
$$

Soit $(J, \Phi)$ (resp. $\left.\left(J_{(0)}, \Phi_{(0)}\right),\left(J_{(\infty)}, \Phi_{(\infty)}\right)\right)$ la jacobienne relative de $\mathbb{P}_{S}^{1}-D$ (resp. $\left.U_{(\infty)}=\mathbb{P}_{S}^{1}-D_{(0)}, U_{(0)}=\mathbb{P}_{S}^{1}-D_{(\infty)}\right)$. (cf. [3], [5]).

Soit ${ }^{(0)} \mathfrak{J}$ (resp. $\left.{ }^{(\infty)} \mathfrak{J}\right)$ le $S$-groupe défini à partir de $B_{(0)}\left(\operatorname{resp} . B_{(\infty)}\right)$ comme dans $\S 0$. Le $S$-homomorphisme (1.2.12) donne lieu à un $S$-homomorphisme

$$
{ }^{(0)} \mathfrak{J} \times{ }^{(\infty)} \mathfrak{J} \longrightarrow J\left(\operatorname{resp} .{ }^{(0)} \mathfrak{J} \longrightarrow J_{(0)},{ }^{(\infty)} \mathfrak{J} \longrightarrow J_{(\infty)}\right)
$$

Dans ce paragraphe on se propose d'expliciter $J$ (resp. $\left.J_{(0)}, J_{(\infty)}\right)$ comme un quotient du groupe de nature locale ${ }^{(0)} \mathfrak{I} \times{ }^{(\infty)} \mathfrak{J}$ (resp. $\left.{ }^{(0)} \mathfrak{J},{ }^{(\infty)} \mathfrak{J}\right)$.

On désigne par $L_{(0)} \subset \mathcal{O}_{X}$ (resp. $\left.L_{(\infty)} \subset \mathcal{O}_{X}, L_{D} \subset \mathcal{O}_{X}\right)$ l'idéal de définition de $D_{(0)}$ (resp. $\left.D_{(\infty)}, D\right)$. On a alors que l'idéal $I_{(0)} \subset B_{(0)}\left(\right.$ resp. $\left.I(\infty) \subset B_{(\infty)}\right)$ est induit par $L_{(0)}\left(\right.$ resp. $\left.L_{(\infty)}\right)$. On identifie $V\left(I_{(0)}\right) \subset \operatorname{omb}\left(\mathfrak{X}_{(0)}\right)=\operatorname{Spec}\left(B_{(0)}\right)$ (resp. $\left.V\left(I_{(\infty)}\right) \subset \operatorname{omb}\left(\mathfrak{X}_{(\infty)}\right)=\operatorname{Spec}\left(B_{(\infty)}\right)\right)$ à $D_{(0)}\left(\operatorname{resp} . D_{(\infty)}\right)$.

Soit $\widetilde{T}$ la fonction méromorphe relative de $X=\mathbb{P}_{S}^{1}$ définie par la section

$$
T_{1} T_{0}^{-1} \in \Gamma\left(U_{(0)}, \mathcal{O}_{X}\right) .
$$

Il est clair que $U_{(0)}$ est le domaine de définition de $\mathbb{T}$.

Soit $S^{\prime}$ un $S$-schéma. On désigne par $\mathfrak{T}_{S}$, la fonction méromorphe relative de $X \times_{S} S^{\prime}=\mathbb{P}_{S}^{1}$, donnée par $\mathfrak{T}$. On vérifie que $\mathfrak{T}$ (resp. $\mathfrak{T}^{-1}$ ) donne lieu à un générateur de $L_{(0)}\left(\right.$ resp. $\left.L_{(\infty)}\right)$ dans l'ouvert $U_{(0)}\left(\right.$ resp. $\left.U_{(\infty)}\right)$. On note par $T$ (resp. $T^{\prime}$ ) le générateur de $I_{(0)} \subset B_{(0)}$ (resp. $I_{(\infty)} \subset B_{(\infty)}$ ) induit par $\mathfrak{T}$ (resp. $\left.\mathfrak{T}^{-1}\right)$.

Posons

$$
\begin{aligned}
\mathfrak{U}_{(0)} & =\operatorname{omb}\left(\mathfrak{X}_{(0)}\right)-D_{(0)}=\operatorname{Spec}\left(B_{(0)}\left[I_{(0)}^{-1}\right]\right) \\
\left(\operatorname{resp} . \mathfrak{U}_{(\infty)}\right. & \left.=\operatorname{omb}\left(\mathfrak{X}_{(\infty)}\right)-D_{(\infty)}=\operatorname{Spec}\left(B_{(\infty)}\left[I_{(\infty)}^{-1}\right]\right)\right) .
\end{aligned}
$$

Soit

$$
\varphi_{T}^{1} \in{ }^{(0)} \mathfrak{J}\left(\mathfrak{U}_{(0)}\right) \quad\left(\operatorname{resp} . \varphi_{T^{\prime}}^{1} \in{ }^{(\infty)} \mathfrak{J}\left(\mathfrak{U}_{(\infty)}\right)\right)
$$

la section définie comme dans (0.31), (iii), à partir du générateur $T$ (resp. $T^{\prime}$ ) 
de $I_{(0)}\left(\operatorname{resp} . I_{(\infty)}\right)$, c.à.d. $\varphi_{T}^{1}\left(\operatorname{resp} . \varphi_{T^{\prime}}^{1}\right)$ est donné par la section

$$
\begin{gathered}
T \otimes 1(1 \otimes T-T \otimes 1)^{-1} \in B_{(0)} \widehat{\otimes}_{A} B_{(0)}\left[I_{(0)}^{-1}\right] \\
\left(\text { resp. } T^{\prime} \otimes 1\left(1 \otimes T^{\prime}-T^{\prime} \otimes 1\right)^{-1} \in B_{(\infty)} \widehat{\otimes}_{A} B_{(\infty)}\left[I_{(\infty)}^{-1}\right]\right) .
\end{gathered}
$$

Soit $X_{U}=X \times_{S} U$. Notons par $t$ la section de $\mathcal{O}_{X_{U}}$ donnée par l'image inverse par $X \times{ }_{S} U \longrightarrow U$ de la restriction de $\mathbb{C}$ à $U \subset U_{(0)}$ (= domaine de définition de $\mathfrak{T}$ ). On définit une fonction méromorphe relative de $X_{U}$ en posant

$$
m_{(0)}=\mathfrak{C}_{U}\left(t-\mathfrak{T}_{U}\right)^{-1} .
$$

REMARque 1.3.9. On a un morphisme évident

$$
\operatorname{omb}\left(\mathfrak{X}_{(0)} \times_{S} \mathfrak{U}_{(0)}\right) \longrightarrow U_{(0)} \times_{S} U .
$$

L'image $m_{(0)}^{\prime}$ de $m_{(0)}$ dans

$$
\Gamma\left(\operatorname{omb}\left(\mathfrak{X}_{(0)} \times_{S} \mathfrak{U}_{(0)}\right), \mathcal{O}_{\text {omb }\left(\mathfrak{x}_{(0)} \times \mathfrak{U}_{(0)}\right)}\right)
$$

donne lieu par restriction à

$$
\operatorname{omb}\left(\mathfrak{x}_{(0)} \times_{S} \mathfrak{U}_{(0)}\right)-D_{(0)} \times_{S} \mathfrak{U}_{(0)}
$$

$\grave{a}$ une section $m^{\prime \prime}{ }_{0}$ qui est en fait une unité. On a ainsi une section de $\underline{\mathrm{G}}_{m}\left(\mathfrak{U}_{(0)}\right)$ au-dessus de $\mathfrak{H}_{(0)}$, et l'on vérifie qu'elle coüncide avec $\varphi_{T}^{1}$.

Soit $L_{U^{\prime}} \subset \mathcal{O}_{X_{U}}$ l'idéal de définition dans $X_{U}$ du sous-schéma $\Delta \cap(X \times U)$. Soit $\operatorname{pr}_{1}: X \times_{S} U \longrightarrow X$ la première projection. La fonction méromorphe relative $m_{(0)}$ donne lieu à un isomorphisme

$$
L_{\Delta^{\prime}} \simeq \operatorname{pr}_{1}^{*}\left(L_{(0)}\right)
$$

que l'on désigne toujours par $m_{(0)}$.

Soient $S^{\prime}$ un $S$-schéma, et $\underline{n} \in \Gamma\left(S^{\prime}, \underline{Z}_{S^{\prime}}\right)$. On désigne par

$$
\operatorname{div}_{(0)}\left(\mathbb{C} \frac{n}{\bar{S}^{\prime}}\right) \quad\left(\operatorname{resp} \cdot \operatorname{div}_{(\infty)}\left(\mathbb{C}_{\bar{S}^{\prime}}^{\prime}\right)\right)
$$

la section de ${ }^{(0)} J \subset \underline{G}_{m S}\left(\mathfrak{U}_{(0)}\right)\left(\right.$ resp. $\left.{ }^{(\infty)} J \subset \underline{G}_{m S}\left(\mathfrak{H}_{(\infty)}\right)\right)$ au-dessus de $S^{\prime}$ donnée par la restriction de la fonction méromorphe relative $\mathfrak{T}_{\bar{S}^{\prime}}^{n}$ à

$$
\begin{gathered}
\left(\mathfrak{U}_{(0)}\right)_{\left\{S^{\prime}\right\}}=\operatorname{omb}\left(\mathfrak{X}_{(0)} \times_{X} S^{\prime}\right)-D_{(0)} \times_{S} S^{\prime} \\
\left(\operatorname{resp.}\left(\mathfrak{U}_{(\infty)}\right)_{\left\{S^{\prime}\right\}}=\operatorname{omb}\left(\mathfrak{X}_{(\infty)} \times_{X} S^{\prime}\right)-D_{(\infty)} \times_{S} S^{\prime}\right) .
\end{gathered}
$$


Remarquer que si l'on désigne par

$$
\varepsilon_{(0)}:{ }^{(0)} \mathfrak{J} \longrightarrow \underline{Z}_{S}\left(\operatorname{resp} . \varepsilon_{(\infty)}:{ }^{(\infty)} \mathfrak{J} \longrightarrow \underline{Z}_{S}\right)
$$

le $S$-homomorphisme d'augmentation défini comme dans (0.9), on a alors

$$
\varepsilon_{(0)}\left(\operatorname{div}_{(0)}\left(\mathbb{T}_{\bar{S}^{\prime}}^{n}\right)\right)=\underline{n}\left(\operatorname{resp} . \varepsilon_{(\infty)}\left(\operatorname{div}_{(\infty)}\left(\mathbb{T}_{\bar{S}^{\prime}}^{n}\right)=-\underline{n}\right)\right) .
$$

On définit un $S$-homomorphisme :

$$
\underline{Z}_{S} \longrightarrow^{(0)} \mathfrak{J} \times{ }^{(\infty)} \mathfrak{J}
$$

en faisant correspondre à une section $\underline{n} \in \Gamma\left(S^{\prime}, \underline{Z}_{S^{\prime}}\right)$ la section $\left(\operatorname{div}_{(0)}\left(\widetilde{T}_{S^{\prime}}^{n}\right), \operatorname{div}_{(\infty)}\left(\widetilde{T}_{S^{\prime}}^{n}\right)\right)$ de ${ }^{(0)} \mathfrak{J} \times{ }^{(\infty)} \mathfrak{J}$ au-dessus de $S^{\prime}$. Soit

$$
\text { Div. } \operatorname{princ}_{D}(X) \subset^{(0)} \mathfrak{J} \times^{(\infty)} \mathfrak{J} .
$$

le $S$-sous-groupe engendré par l'image de (1.3.11) et par celle du morphisme diagonal

$$
\underline{\mathrm{G}} \longrightarrow{ }^{(0)} \mathfrak{J} \times{ }^{(\infty)} \mathfrak{J} .
$$

(Les composantes de ce dernier sont respectivement les $S$-homomorphismes

$$
\underline{\mathrm{G}}_{\mathrm{mS}} \longrightarrow \underline{\mathrm{G}}_{\mathrm{mS}}\left(\mathfrak{X}_{(0)}\right)={ }^{(0)} \mathfrak{J} \text { et } \underline{\mathrm{G}}_{\mathrm{mS}} \longrightarrow \underline{\mathrm{G}}_{\mathrm{mS}}\left(\mathfrak{X}_{(\infty)}\right)={ }^{(\infty)} \mathfrak{J}
$$

induits respectivement par $\mathfrak{X}_{(0)} \longrightarrow S$ et $\mathfrak{X}_{(\infty)} \longrightarrow S$ ).

On pose

$$
\begin{gathered}
{ }^{(0)} \wedge^{0}=\operatorname{Ker}\left({ }^{(0)} \mathfrak{J}^{0} \longrightarrow \underline{\mathrm{G}} \mathrm{mS}\right. \\
(\text { resp. } \\
\left.{ }^{(\infty)} \wedge^{0}=\operatorname{Ker}\left({ }^{(\infty)} \mathfrak{J}^{0} \longrightarrow \underline{\mathrm{G}}_{\mathrm{mS}}\right)\right)
\end{gathered}
$$

et

$$
{ }^{(0)} \wedge={ }^{(0)} \mathfrak{J} / \underline{\mathrm{G}}_{\mathrm{mS}}\left(\operatorname{resp} .{ }^{(\infty)} \wedge^{0}={ }^{(\infty)} \mathfrak{J} / \underline{\mathrm{G}}_{\mathrm{mS}}\right)
$$

A partir d'un scindage de $\varepsilon_{(0)}$ (resp. $\varepsilon_{(\infty)}$ ) on construit un $S$-isomorphisme

$$
{ }^{(0)} \wedge \simeq^{(0)} \wedge^{0} \times \underline{Z}_{S} \quad\left(\text { resp. }{ }^{(\infty)} \wedge \simeq^{(\infty)} \wedge^{0} \times \underline{Z}_{S}\right) .
$$

LEMME 1.3.14. Avec les notations ci-dessus on a une décomposition en produit direct

${ }^{(0)} \mathfrak{J} \times{ }^{(\infty)} \mathfrak{J} \simeq$ Div. $\operatorname{princ}_{D}(X) \times\left({ }^{(0)} \mathfrak{J} \times{ }^{(\infty)} \wedge^{0}\right)$. 
Preuve. Soit $u=\left(u_{(0)}, u_{(\infty)}\right)$ une section de ${ }^{(0)} \mathfrak{I} \times{ }^{(\infty)} \mathfrak{I}$ au-dessus de $S^{\prime}$. Posons

$$
u_{(\infty)}^{\prime} u_{(\infty)}^{\prime \prime}=u_{(\infty)} \operatorname{div}_{(\infty)}\left(\widetilde{T}_{S^{\prime}}\right)^{\varepsilon_{(\infty)}}\left(U_{(\infty)}\right)
$$

$\operatorname{avec} u_{(\infty)}^{\prime} \in \Gamma\left(S^{\prime}, \underline{\mathrm{G}}_{m S}\right)$, et $u_{(\infty)}^{\prime \prime} \in \Gamma\left(S^{\prime},{ }^{(\infty)} \wedge^{0}\right)$.

Soit $v=\left(v_{(0)}, v_{(\infty)}\right)$ défini par:

$$
v_{(0)}=u_{(\infty)}^{\prime} \operatorname{div}_{(0)}\left(\boldsymbol{T}_{S^{\prime}}\right)^{-\varepsilon_{(\infty)}\left(u_{(\infty)}\right.}
$$

et

$$
v_{(\infty)}=u_{(\infty)}^{\prime} \operatorname{div}_{(\infty)}\left(\mathfrak{T}_{S^{\prime}}\right)^{-\varepsilon_{(\infty)}\left(u_{(\infty)}\right.} .
$$

Il est clair que l'on a $v \in \Gamma$ (S', Div.princ $\left.{ }_{D}(X)\right)$ et que $u v^{-1} \in$ $\Gamma\left(S^{\prime},{ }^{(0)} \mathfrak{I} \times{ }^{(\infty)} \wedge^{0}\right)$. On peut alors écrire

$$
u=v\left(u v^{-1}\right) .
$$

On vérifie que l'on définit ainsi une décomposition en produit direct de $(0) \mathfrak{J} \times(\infty) \mathfrak{J}$.

C.Q.F.D.

Notons par $\underline{1}_{0}$ (resp. $\underline{1}_{\infty}$ ) la trivialisation du complété formel

$$
\left(L_{\Delta^{\prime}}\right)_{0}^{\wedge}\left(\operatorname{resp} .\left(L_{\Delta^{\prime}}\right)_{\infty}^{\wedge}\right)
$$

de $L_{\Delta^{\prime}}$ le long de $D_{(0)} \times_{S} U$ (resp. $\left.D_{(\infty)} \times_{S} U\right)$ donnée par

$$
\begin{gathered}
\underline{1} \in \Gamma\left(\mathfrak{X}_{(0)} \times_{S} U, \mathcal{O}_{\mathfrak{X}_{(0)} \times_{S} U}\right)=\Gamma\left(\mathfrak{X}_{(0)} \times_{S} U,\left(L_{\Delta^{\prime}}\right)_{0}^{\wedge}\right) \\
\left(\text { resp. } \underline{1} \in \Gamma\left(\mathfrak{X}_{(\infty)} \times_{S} U, \mathcal{O}_{\mathfrak{X}_{(\infty)} \times_{S} U}\right)=\Gamma\left(\mathfrak{X}_{(\infty)} \times_{S} U,\left(L_{\Delta^{\prime}}\right)_{\infty}^{\wedge}\right)\right) .
\end{gathered}
$$

Le couple $\left(\underline{1}_{0}, \underline{1}_{\infty}\right)$ correspond à une trivialisation du complété formel $\left(L_{\Delta^{\prime}}\right)^{\wedge}$ de $L_{\Delta^{\prime}}$ le long de $D_{U}=D \times_{S} U$. On a alors que la classe d'isomorphisme de $\left(L_{\Lambda^{\prime}},\left(\underline{1}_{0}, \underline{1}_{\infty}\right)\right)$ donne lieu à une section de $J$ au-dessus de $U=\mathbb{P}_{S}^{1}-D$, qui coïncide avec $\Phi$. En vertu de l'isomorphisme (1.3.10) on a alors que la section de $J$ correspondante au couple

$$
\left(\operatorname{pr}_{1}^{*}\left(L_{(0)}\right),\left(m_{(0)}\left(\underline{1}_{0}\right), m_{(0)}\left(\underline{1}_{\infty}\right)\right)\right)
$$

est égale à $\Phi$. On vérifie que

$$
m_{(0)}\left(\underline{1}_{0}\right)=T(t-T)^{-1} \in{ }^{(0)} \mathfrak{J}(U)
$$

$$
\text { (resp. } \left.m_{(0)}\left(\underline{1}_{\infty}\right)=\left(T^{\prime} t-1\right)^{-1} \in{ }^{(\infty)} \mathfrak{J}^{0}(U)\right) \text {. }
$$


REMARQUe 1.3.16. Par (1.3.9) on a que la restriction de $m_{(0)}\left(\underline{1}_{0}\right) \grave{a}$ $\operatorname{omb}\left(\mathfrak{X}_{(0)} \times_{S} \mathfrak{U}_{(0)}\right)-D_{0} \times_{S} \mathfrak{U}_{(0)}$ coïncide avec la section $\varphi_{T}^{1}$ de ${ }^{(0)} \mathfrak{J}^{1}$ au-dessus de $\mathfrak{H}_{(0)}$. Ce dernier s'identifiant au ${ }^{(0)} \mathfrak{J}^{0}$-torseur des trivialisations de $L_{(0)}$.

Il est clair que Div. $\operatorname{princ}_{D}(X)$ est contenu dans le noyau de (1.3.7). On a alors à partir de (1.2.4) et de (1.3.7) un diagramme commutatif aux lignes exactes.

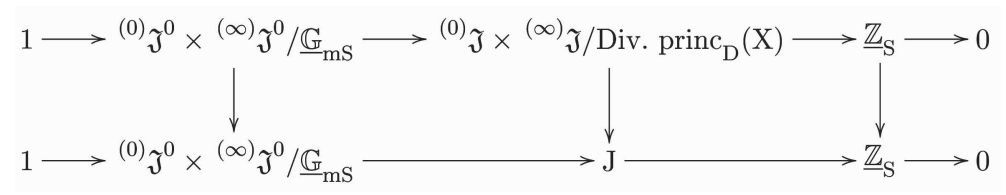

D'où on a le

LEMme 1.3.18. Le S-homomorphisme (1.3.7) induit, par passage au quotient un S-isomorphisme

$$
{ }^{(0)} \mathfrak{J} \times{ }^{(\infty)} \mathfrak{J} / \text { Div. } \operatorname{princ}_{D}(X) \simeq J,
$$

et à fortiori un S-isomorphisme

$$
{ }^{(0)} \mathfrak{J} \times{ }^{(\infty)} \wedge^{0} \simeq J
$$

D'autre part on vérifie que la ${ }^{(0)} \mathfrak{J} \times{ }^{(\infty)} \wedge^{0}$-composante de $\left.\left(m_{(0)} \underline{1}_{0}\right), m_{(0)}\left(\underline{1}_{\infty}\right)\right)$ donnée par la décomposition en produit direct (1.3.14) est égale à

$$
\left(-T(t-T)^{-1},\left(1-t T^{\prime}\right)^{-1}\right)
$$

En résumé on a la

Proposition 1.3.19. Le S-homomorphisme (1.3.7) induit un isomorphisme ${ }^{(0)} \mathfrak{J} \times{ }^{(\infty)} \wedge^{0} \simeq J$, faisant correspondre à la section

$$
\left(-T(t-T)^{-1},\left(1-t T^{\prime}\right)^{-1}\right) \in\left({ }^{(0)} \mathfrak{J} \times{ }^{(\infty)} \wedge^{0}\right)(U)
$$

la section $\Phi \in J(U)$.

Le choix d'un générateur $T$ de $I_{(0)}\left(\operatorname{resp} . T^{\prime}\right.$ de $\left.I_{(\infty)}\right)$ donne lieu à un isomorphisme

${ }^{(0)} \wedge={ }^{(0)} \mathfrak{J} / \underline{G}_{m S} \simeq^{(0)} \wedge^{0} \times \underline{Z}_{S} \quad\left(\right.$ resp. $\left.{ }^{(\infty)} \wedge==^{(\infty)} \mathfrak{J} / \underline{G}_{m S} \simeq^{(\infty)} \wedge^{0} \times \underline{Z}_{S}\right)$. 
On a alors la

Proposition 1.3.20. Le S-homomorphisme (1.3.7) induit un isomorphisme

$$
{ }^{(0)} \wedge^{0} \times \underline{Z}_{S} \simeq^{(0)} \wedge \simeq J_{(0)}\left(\operatorname{resp} . \quad{ }^{(\infty)} \wedge^{0} \times \underline{Z}_{S} \simeq^{(\infty)} \wedge \simeq J_{(0)}\right)
$$

tel que la section $\left(T\left(1-t^{-1} T\right)^{-1} \in{ }^{(0)} \wedge(U)\right.$ (resp. $\left(T^{\prime}\left(1-t^{-1} T^{\prime}\right)^{-1} \in(\infty)\right.$ $\left.\wedge^{0}(U)\right)$ correspond à $\Phi_{(0)}\left(r e s p . \Phi_{(\infty)}\right)$.

L'inclusion $U \longrightarrow U_{(0)}$ (resp. $\left.U \longrightarrow U_{(\infty)}\right)$ donne lieu à un $S$-homomorphisme

$$
p_{(\infty)}: J \longrightarrow J_{(\infty)} \quad\left(\operatorname{resp} \cdot p_{(0)}: J \longrightarrow J_{(0)}\right),
$$

tel que si l'on note par $\Phi_{(\infty)}^{\prime}\left(\right.$ resp. $\left.\Phi_{(0)}^{\prime}\right)$ la restriction de $\Phi_{(\infty)}\left(\right.$ resp. $\left.\Phi_{(0)}\right)$ à $U \subset U_{(0)}\left(\right.$ resp. $\left.U \subset U_{(\infty)}\right)$ on a

$$
\Phi_{(\infty)}^{\prime}=p_{(\infty)} \circ \Phi\left(\operatorname{resp} . \Phi_{(0)}^{\prime}=p_{(0)} \circ \Phi\right) .
$$

Avec les identifications de (1.3.19) et de (1.3.20) on vérifie que $p_{(\infty)}$ correspond à la projection canonique

$$
{ }^{(0)} \mathfrak{J} \times{ }^{(\infty)} \wedge^{0} \simeq \underline{Z}_{S} \times{ }^{(0)} \mathfrak{J}^{(0)} \times{ }^{(\infty)} \wedge^{0} \longrightarrow \underline{Z}_{S} \times{ }^{(\infty)} \wedge^{0},
$$

et $p_{(0)}$ à la projection canonique

$$
{ }^{(0)} \mathfrak{J} \times{ }^{(\infty)} \wedge^{0}\left(\simeq \underline{\mathrm{G}}_{\mathrm{mS}} \times^{(0)} \wedge \times^{(\infty)} \wedge^{0}\right) \longrightarrow{ }^{(0)} \wedge .
$$

Notation 1.3.25. Notons par $\varphi_{\mathfrak{\mathbb { C }}}^{1}$ (resp. $\varphi_{\mathfrak{C}}^{0}$ ) la section de ${ }^{(0)} \mathfrak{J}$ (resp. $\left.{ }^{(0)} \mathfrak{J}^{0}\right)$ au-dessus de $U$ donnée par la restriction de la fonction méromorphe relative $m_{(0)}^{1}=\mathfrak{C}_{U}\left(t-\mathfrak{T}_{U}\right)^{-1}$ (resp. $\left.m_{(0)}^{0}=\left(t-\mathfrak{T}_{U}\right)^{-1}\right) \grave{a}$

$$
\left(\mathfrak{U}_{(0)}\right)_{\{U\}}=\operatorname{omb}\left(\mathfrak{X}_{(0)} \times_{S} U\right)-D_{(0)} \times_{S} U \text {. }
$$

( Cette restriction donne lieu à une section de $\underline{G}_{m S}\left(\mathfrak{H}_{(0)}\right)$ au-dessus de $U$ qui appartient en fait $\grave{a}^{(0)} \mathfrak{J}(U)$.)

La propriété universelle de $(J, \Phi)$ entraîne l'existence d'un $S$-homomorphisme unique

$$
q_{(0)}: J \longrightarrow{ }^{(0)} \mathfrak{J}
$$

vérifiant :

$$
\varphi_{\mathfrak{C}}^{1}=q_{(0)} \circ \Phi .
$$


REMARQue 1.3.28. (i) - Compte tenu de (1.3.19) on voit que $q_{(0)}$ est le $S$ homomorphisme ${ }^{(0)} \mathfrak{I} \times{ }^{(\infty)} \wedge^{0} \longrightarrow{ }^{(0)} \mathfrak{I}$ obtenu par composition de la projection canonique

$$
{ }^{(0)} \mathfrak{J} \times{ }^{(\infty)} \wedge^{0} \longrightarrow{ }^{(0)} \mathfrak{J}
$$

avec l'automorphisme $\beta$ de ${ }^{(0)} \mathfrak{J}$ faisant correspondre à la section $T_{u}^{n}$ de ${ }^{(0)} \mathfrak{J}$ la section $T^{n}(-1)^{n} u$.

(ii) - Soit $G$ un S-groupe. Soit $\alpha \in G\left(\mathfrak{H}_{(0)}\right)$ tel qu'il existe un S-homomorphisme

$$
f:{ }^{(0)} \mathfrak{J} \longrightarrow G\left(\operatorname{resp} . f:{ }^{(0)} \mathfrak{J}^{0} \longrightarrow G\right)
$$

vérifiant $\alpha=f \circ \varphi_{T}^{1}$ (resp. $\alpha=f \circ \varphi_{T}^{0}$ ). On a alors une extension $\bar{\alpha}$ de $\alpha: \mathfrak{U}_{(0)} \longrightarrow G \grave{a} U$ en posant

$$
\bar{\alpha}=f \circ \varphi_{\mathfrak{C}}^{1}\left(\operatorname{resp} . \bar{\alpha}=f \circ \varphi_{\mathfrak{C}}^{0}\right)(\operatorname{cf} .(1.3 .9)) .
$$

Soit $q_{(0)}^{0}: J^{0} \longrightarrow{ }^{(0)} \mathfrak{J}^{0}$ le composé de $q_{(0)}$ avec la projection canonique ${ }^{(0)} \mathfrak{J}=Z_{x} \times{ }^{(0)} \mathfrak{J}^{0} \longrightarrow{ }^{(0)} \mathfrak{J}^{0}$. Compte tenu de (1.3.27) on trouve

$$
\bar{\alpha}=\left(f \circ q_{(0)}\right) \circ \Phi\left(\text { resp. } \bar{\alpha}=\left(f \circ q_{(0)}^{0}\right) \circ \Phi\right) .
$$

Proposition 1.3.29. Soit $A$ un anneau. On pose $S=\operatorname{Spec}(A)$. Soit $G$ un $S$-schéma en groupes lisse et séparé. Soit $h \in H_{0 m_{-g r}}\left({ }^{(0)} \mathfrak{J}, G\right)$. Posons $\psi=h \circ \varphi_{\mathfrak{C}}^{1} \in{ }^{(0)} \mathfrak{I}(U)$. On suppose que $\psi$ admet un prolongement $\bar{\psi} \grave{a}$ $\mathbb{P}_{S}^{1}-D_{(\infty)}$.

Alors le morphisme $\psi$ est constant, c.à.d. il existe une section $\sigma: S \longrightarrow G$ au-dessus de $S$ tel que $\psi$ s'obtient par composition de $\mathrm{P}_{S}^{1} \longrightarrow S$ avec $\sigma$.

PREUVE. Le morphisme $\psi$ admet la factorisation suivante

$$
\psi=h \circ\left(q_{(0)} \circ \Phi\right)(\mathrm{cf} .(1.3 .27)) .
$$

Puisque $\psi$ se prolonge à $\mathbb{P}_{S}^{1}-D_{(\infty)}$ on peut trouver $h^{\prime} \in \operatorname{Hom}_{S-g r}\left(J_{(\infty)}, G\right)$ tel que

$$
\psi=\left(h^{\prime} \circ p_{(\infty)}\right) \circ \Phi(\mathrm{cf} .(1.3 .22)) .
$$

(En fait on $\left.a \bar{\psi}=h^{\prime} \circ \Phi_{(\infty)}\right)$. L'unicité de la factorisation de la section $\psi$ de Gau-dessus de $U$ donne l'égalité

$$
h^{\prime} \circ p_{(\infty)}=h \circ q_{(0)} .
$$

Posons $g=h^{\prime} \circ p_{(\infty)}=h \circ q_{(0)}$. D'autre par d'après (1.3.22) on a que ${ }^{(0)} \mathfrak{J}^{0} \subset \operatorname{Ker} p_{(\infty)}$, et d'après (1.3.28) (i), on a ${ }^{(\infty)} \wedge^{0} \subset \operatorname{Ker} q_{(0)}$. D'où $i l$ 
résulte que

$$
{ }^{(0)} \mathfrak{J}^{0} \times{ }^{(\infty)} \wedge^{0} \subset \operatorname{Ker}(g) .
$$

Puisque d'après (1.3.19) la section $\Phi$ correspond par l'isomorphisme ${ }^{(0)} \mathfrak{J}^{0} \times \underline{Z}_{S} \times{ }^{(\infty)} \wedge^{0} \simeq J$ à la section

$$
\left(-T(t-T)^{-1},\left(1-t T^{\prime}\right)^{-1}\right),
$$

on a que la $\underline{Z}_{S}$-composante de $\Phi$ est égale à $T$. Il en résulte que $\psi=g \circ \Phi$ admet un prolongement $\overline{\bar{\psi}} \grave{a} \mathrm{P}_{S}^{1}$. Donc $\overline{\bar{\psi}}$ est constant, c.à.d. il existe une section $\sigma$ de G au-dessus de $S$ tel que $\overline{\bar{\psi}}$ correspond à l'image inverse de $\sigma$ $\operatorname{par} \mathbb{P}_{S}^{1} \longrightarrow S$.

C.Q.F.D.

(1.4) On reprend dans ce numéro les notations de $\S 0$.

Soit $G$ un $S$-groupe. On pose

$$
G(\mathfrak{X})^{+}=\operatorname{Ker}(G(\operatorname{omb}(\mathfrak{X})) \longrightarrow G(S)),
$$

et

$$
F(G)=G(\mathfrak{U}) / G(\operatorname{omb}(\mathfrak{X}))\left(\operatorname{resp} . F^{+}(G)=G(\mathfrak{U}) / G(\mathfrak{X})^{+}\right) .
$$

Soient $\varphi^{0}$ et $\varphi^{1}$ comme dans (0.32). On a alors par composition avec $\varphi^{0}$ (resp. $\varphi^{1}$ ) un homomorphisme de groupes

(1.4.3) $\rho: \operatorname{Hom}_{S-g r}\left(\mathfrak{J}^{0}, G\right) \longrightarrow F(G)\left(\operatorname{resp} . \rho^{+}: \operatorname{Hom}_{S-g r}(\mathfrak{J}, G) \longrightarrow F^{+}(G)\right)$. Le couple $\left(\mathfrak{J}^{0}, \varphi^{0}\right)$ (resp. $\left.\left(\mathfrak{J}, \varphi^{1}\right)\right)$ vérifie la propriété universelle suivante

ThÉORÈme 1.4.4. On reprend les notations ci-dessus. Soit $A$ un anneau noethérien et posons $S=\operatorname{Spec}(A)$. Soit $G$ un $S$-schéma en groupes commutatif, lisse et séparé. On a alors $\rho$ et $\rho^{+}$sont des isomorphismes.

La preuve de ce théorème est donnée dans les paragraphes $\S 2, \S 3, \S 4$ et $\S 5$. Commençons par démontrer la

Proposition 1.4.5. Soit $A$ una anneau. On pose $S=\operatorname{Spec}(A)$. Soit $G$ un $S$-schéma en groupes comme dans (1.4.4). Alors les homomorphismes $\rho$ et $\rho^{+}$sont injectifs.

Preuve. Reprenons ici les notations de (1.3). Sans perte de généralité on peut supposer que $B=B_{(0)}, \mathfrak{X}=\mathfrak{X}_{(0)}, \mathfrak{U}=\mathfrak{U}_{(0)}, \mathfrak{J}^{0}={ }^{(0)} \mathfrak{I}^{0}$ et $\mathfrak{I}={ }^{(0)} \mathfrak{I}$, et que $\rho^{+}$(resp. $\left.\rho\right)$ est obtenu par composition avec $\varphi_{T}^{1}$ (resp. $\left.\varphi_{T}^{0}\right)$. Pour 
montrer que $\rho^{+}$(resp. $\left.\rho\right)$ est injuectif il suffit donc de voir que quel que soit $h \in \operatorname{Hom}_{S-g r}\left({ }^{(0)} \mathfrak{J}, G\right)$ (resp. $\left.h \in \operatorname{Hom}_{S-g r}\left({ }^{(0)} \mathfrak{J}^{0}, G\right)\right)$ ) tel que la section $h \circ \varphi_{T}^{1}$ de $G$ au-dessus de $\mathfrak{U}_{(0)}$ admet un prolongement à $G($ omb $(\mathfrak{X}))$, et en plus appartient à l'image de

$$
\operatorname{Ker}(G(o m b(\mathfrak{X})) \longrightarrow G(S))
$$

on $a \mathrm{~h}=0$ (resp. tel que la section $h \circ \varphi_{T}^{0}$ de $G$ au-dessus de $\mathfrak{H}_{(0)}$ admet un prolongement à $G(\mathrm{omb}(\mathfrak{X}))$ alors $h=0)$. Voyons que $\rho^{+}$est injectif. Montrons que pour cela il suffit de prouver que $\psi=h \circ \varphi_{\mathbb{T}}^{1}$ admet un prolongement $\bar{\psi} \grave{a} \mathbb{P}_{S}^{1}-D_{(\infty)}$. On reprend ici les notations de la preuve de (1.3.29). On peut écrire $\psi=g \circ \Phi$ avec $g: J \longrightarrow G$ qui se factorise par $J \longrightarrow \underline{Z}_{S}$. Le prolongement $\overline{\bar{\psi}}$ de $\psi$ à $\mathrm{P}_{S}^{1}$ s'obtient alors comme le composé de $\mathbb{P}_{S}^{1} \longrightarrow S$, de $S \longrightarrow \underline{\mathbb{Z}}_{S}$ donné par $\underline{1}$, et du homomorphisme $\underline{\mathbb{Z}}_{S} \longrightarrow G$ induit par g. L'hypothèse $h \circ \varphi_{T}^{1} \in \operatorname{Ker}(G(\operatorname{omb}(\mathfrak{X}))) \longrightarrow G(S)$ entraîne que la restriction de $\overline{\bar{\psi}} \grave{a} D_{(0)}$ est donnée par la section élément neutre de $G$, d'où que $g=0$ et a fortiori que $h=0$ vu (1.3.28)(i).

Prouvons que $\psi$ admet un prolongement $\bar{\psi}$ à $\mathrm{P}_{S}^{1}-D_{(\infty)}$. Supposons d'abord que A est noethérien. Dans ce cas on a le morphisme fpqc suivant

$$
\operatorname{omb}\left(\mathfrak{x}_{(0)}\right) \coprod U \longrightarrow \mathbb{P}_{S}^{1}-D_{(\infty)} \text {. }
$$

Comme la restriction de $\psi$ à $\mathfrak{U}_{(0)}$ est égale à $h \circ \varphi_{T}^{1}$, et par hypothèse la section $h \circ \varphi_{T}^{1}$ de $G$ au-dessus de $\mathfrak{H}_{(0)}$ admet un prolongement à omb $\left(\mathfrak{X}_{(0)}\right)$, il en résulte que $\psi$ admet un prolongement $\bar{\psi} \grave{a} \mathbb{P}_{S}^{1}-D_{(\infty)}$.

Soit $A$ un anneau quelconque. Pour tout $x \in \operatorname{Spec}(A)$ soit $h_{K(x)} l a$ fibre en Spec $(K(x))$ de $h$, où $K(x)$ désigne le corps résiduel de $A$ en $x$. L'hypothèse entraîne que $h_{K(x)} \circ \varphi_{T}^{1} \in G\left(\left(\mathfrak{U}_{(0)}\right)_{\{K(x)\}}\right)$ appartient à $\operatorname{Ker}\left(G\left(\operatorname{omb}\left(\mathfrak{X}_{K(x)}\right)\right) \longrightarrow G(K(x))\right)$. Le cas noethérien appliqué pour $A=K(x)$ entraîne que $h_{K(x)}$ est le morphisme 0, donc que l'image (ensembliste) de h est la section unité de G. Ainsi quitte à localiser Spec (A) on peut supposer que $h$ se factorise par un ouvert affine $W \subset G$ voisinage de la section unité. L'hypothèse $h \circ \varphi_{T}^{1} \in G(o m b(\mathfrak{X}))$ jointe au fait que $A[[T]] \cap A\left[T, T^{-1}\right]=A[T]$ implique qu'il existe un prolongement $\bar{\psi} \grave{a}$ $\mathbb{P}^{1}-D_{(\infty)}$ de $\psi$. Ce qui achève la preuve de la proposition. $\quad$ C.Q.F.D.

Soit $S^{\prime}=\operatorname{Spec}\left(A^{\prime}\right) \longrightarrow S$ un $S$-schéma affine.

Soient

$$
\rho^{\prime}: \operatorname{Hom}_{S^{\prime}-\mathrm{gr}}\left(\mathfrak{J}_{S^{\prime}}^{0}, G_{S^{\prime}}\right) \longrightarrow F\left(G_{S^{\prime}}\right)
$$

et

$$
\left(\rho^{+}\right)^{\prime}: \operatorname{Hom}_{S^{\prime}-\mathrm{gr}}\left(\mathfrak{J}_{S^{\prime}}, G_{S^{\prime}}\right) \longrightarrow F^{+}\left(G_{S^{\prime}}\right)
$$

définis comme dans (1.4.3). 
L’injectivité de $\rho$ et $\rho^{+}$entraîne par descente fidèlement plate la proposition suivante.

Proposition 1.4.6. On reprend les notations ci-dessus et on suppose que $S^{\prime}=\operatorname{Spec}\left(A^{\prime}\right)$ est un $S$-schéma fidèlement plat. Soit G un S-schéma en groupes commutatifs, lisse et séparé. On a alors que si $\rho^{\prime}$ et $\left(\rho^{+}\right)^{\prime}$ sont des isomorphismes de groupes $\rho$ et $\rho^{+}$le sont aussi.

(1.5) Dans ce numéro on suppose que $S$ est noethérien. Les constructions suivantes ont pour but d'associer au $S$-groupe $\breve{W}$ un $S$-foncteur $\operatorname{omb}(\breve{W})$, qui s'obtient comme la limite inductive d'un ind-objet de Sch $\left.\right|_{S}$ si $S$ est affine, et en général comme la limite inductive d'un système inductif de $S$-espaces localement annelés, et au $S$-groupe $\Im$ un $S$-foncteur $\Im_{\text {omb }}$ qui est un $\mathfrak{J}$-torseur de Zariski au-dessus de omb( $\check{W})$. Pour effectuer ces constructions on supposera que $S$ est affine bien qu'elles gardent un sens en général.

On met à profit que $\check{W}$ est isomorphe à la limite inductive $\lim _{\vec{n}} W_{n}$ (cf. (0.44)) pour définir omb( $(\breve{W})$. Noter que si $S$ est un schéma noethérien (resp. noethérien affine) alors $W_{n}$ est un $S$-schéma formel noethérien (resp. noethérien affine). Soit omb $\left(W_{n}\right)$ l'espace localement annelé au-dessus de $S$ qui lui correspond par (0.53). Si $S$ est affine $\operatorname{omb}\left(W_{n}\right)$ est un $S$-schéma affine. Pour tout couple d'entiers $0<m \leq n$ on note par

$$
i_{m n}: \operatorname{omb}\left(W_{m}\right) \longrightarrow \operatorname{omb}\left(W_{n}\right)
$$

le morphisme donné par $W_{m} \longrightarrow W_{n}$ (cf. (0.42)). Soit omb( $\left.\breve{W}\right)$ le $S$-faisceau de Zariski défini par le $S$-foncteur

$$
T \longrightarrow \lim _{\vec{n}} \operatorname{Hom}_{S}\left(T, \operatorname{omb}\left(W_{n}\right)\right),
$$

c'est-à-dire $\operatorname{omb}(\check{W})$ est alors la limite inductive du système inductif $\left(\operatorname{omb}\left(W_{n}\right),\left(i_{m n}\right)\right)$. Pour tout entier $n>0$ on a un $S$-morphisme d'immersion

$$
i_{W_{n}}: W_{n} \longrightarrow \operatorname{omb}(\check{W}) .
$$

Soit

$$
i_{\check{W}}: \check{W}=\lim _{\vec{n}} W_{n} \longrightarrow \operatorname{omb}(\check{W})
$$

le $S$-morphisme défini par $\left(i_{W_{n}}\right)$. 
Lemme 1.5.5. (i) Soit Z un S-schéma de présentation finie séparé. Soit

$$
h=\left(h_{n}\right)_{n>0}: \check{W}=\underset{\vec{n}}{\lim } \check{W}_{n} \longrightarrow Z
$$

un S-morphisme, c'est-à-dire un morphisme de S-schémas formels quand on regarde $Z$ comme un S-schéma formel. Il existe alors un unique $S$ morphisme

$$
o m b(h): \operatorname{omb}(\check{W}) \longrightarrow Z
$$

tel que $h=o m b(h) \circ i_{\check{W}}$.

(ii) Soient YY un S-schéma formel noethérien et $k: \mathfrak{Y} \longrightarrow \breve{W}$ un $S$ morphisme qui se factorise à travers $i_{W_{n}}: W_{n} \longrightarrow W_{W}$ pour $n$ convenable.

Alors il existe un unique morphisme omb $(k): o m b(\mathfrak{Y}) \longrightarrow o m b(\check{W})$ tel que $\mathfrak{Y} \longrightarrow o m b(\mathfrak{Y}) \longrightarrow o m b(\check{W})$ soit égal au composé $k \circ i_{\breve{W}}$.

REMARQUE 1.5.6. La limite inductive $\underset{\vec{n}}{\lim } \operatorname{omb}\left(W_{n}\right)$ existe dans la catégorie Locann $\left.\right|_{S}$ des S-espaces localement annelés et on la note par $O m b(\check{W})$. Soit $h_{X}$ le $S$-foncteur défini par un espace localement annelé $X$. Omb( $\check{W})$ vérifie la propriété universelle suivante : il existe une bijection

$$
\operatorname{Hom}_{S}\left(\operatorname{omb}(\check{W}), h_{X}\right) \simeq \operatorname{Hom}_{\text {Locann }_{S}}(\operatorname{Omb}(\check{W}), X) .
$$

LEMme 1.5.7. Soit G un S-schéma en groupes séparé de type fini. Soit $\mathfrak{Z}$ un S-schéma formel noethérien. Soit $\tau$ un G-torseur trivialisable au-dessus de $\mathfrak{Z}$. Il existe alors un $G$-torseur $\tau_{\text {omb }}$ sur omb( $\left.\mathfrak{Z}\right)$ et un morphisme de $G$ torseurs $j_{\tau}: \tau \longrightarrow \tau_{\text {omb }}$ au-dessus de $i_{\mathfrak{Z}}: \mathfrak{Z} \longrightarrow$ omb $(\mathfrak{Z})$ tel que :

(i) Pour tout diagramme commutatif de S-foncteurs

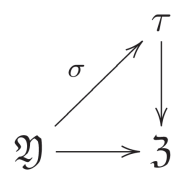

où YY désigne un S-schéma formel affine noethérien, on a un unique diagramme commutatif

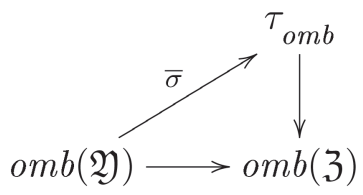

tel que

$$
j_{\tau} \circ \sigma=\bar{\sigma} \circ i_{\mathfrak{Y}}
$$


En particulier on a une bijection

$$
\Gamma(\mathfrak{Z}, \tau) \simeq \Gamma\left(\operatorname{omb}(\mathfrak{Z}), \tau_{\text {omb }}\right) .
$$

(ii) Le diagramme suivant est cartésien

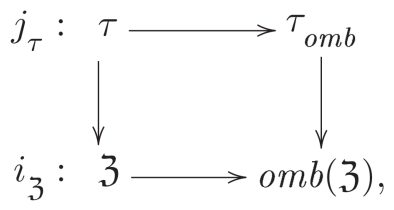

c.à.d. on récupère $\tau$ comme le complété formel de $\tau_{\text {omb }}$ le long du sousschéma fermé de omb(Z) donné par $\mathfrak{Z}$.

Preuve. Étant données deux sections $\sigma$ et $\sigma^{\prime}$ de $\tau$ au-dessus de $\mathfrak{Z}$, soit $d\left(\sigma, \sigma^{\prime}\right) \in G(\mathfrak{Z})$ défini par :

$$
d\left(\sigma, \sigma^{\prime}\right) \sigma^{\prime}=\sigma .
$$

Soit $\left(G \times_{S} \mathfrak{Z},\left(d\left(\sigma, \sigma^{\prime}\right)\right)\right)$ le système inductif de G-torseurs au-dessus de $\mathfrak{Z}$, qui associe $G \times_{S} \mathfrak{Z}$ à $\sigma$, et dont les morphismes de transition correspondent à la multiplication par $d\left(\sigma, \sigma^{\prime}\right)$. Il est clair que l'on a un isomorphisme canonique de G-torseur au-dessus de $\mathfrak{Z}$

$$
\tau \simeq \lim _{\vec{\sigma}} G \times_{S} \mathfrak{Z}(\sigma \in \Gamma(\mathfrak{Z}, \tau)) .
$$

On définit

$$
\tau_{\text {omb }} \simeq \lim _{\vec{\sigma}} G \times_{S} \operatorname{omb}(\mathfrak{Z})(\sigma \in \Gamma(\mathfrak{Z}, \tau))
$$

où les morphismes de transition sont donnés par

$$
\left(\operatorname{omb}\left(d\left(\sigma, \sigma^{\prime}\right)\right)\right) \quad\left(\sigma, \sigma^{\prime} \in \Gamma(\mathfrak{Z}, \tau)\right) .
$$

Les propriétés (i) et (ii) de $\tau_{\text {omb }}$ résultent facilement de sa définition.

C.Q.F.D.

Dans le cas où $B \simeq A[[T]]$ on peut appliquer la construction de (1.5.7) au $\mathfrak{J}^{0}\left(\right.$ resp. $\mathfrak{I}, \mathfrak{J}_{n}^{0}, \mathfrak{I}_{n}$ )-torseur ${ }^{(m)} \Im^{0}\left(\right.$ resp. $\left.{ }^{(m)} \Im,{ }^{(m)} \Im_{n}^{0},{ }^{(m)} \Im_{n}\right)($ cf. $(0.45))$ car il est trivialisable. Avec les notations de (0.47) on pose

$$
\left({ }^{(m)} \Im_{n}^{0}\right)_{\mathrm{omb}}=\lim _{\vec{m}} \mathfrak{J}_{n}^{0} \times \operatorname{omb}\left(W_{m}\right),
$$

où les morphismes de transition sont donnés par $\left(\operatorname{omb}\left({ }^{(m)} h(U, V)_{n}\right)\right)$ 
(cf. (0.47)). (Noter que la donnée d'un générateur $U$ de $I$ entraîne un scindage, $\sigma_{U}$ (cf. (0.46)) et

$$
{ }^{(m)} \Im_{\mathrm{omb}}^{0}=\lim _{\overleftarrow{n}}\left({ }^{(m)} \Im_{n}^{0}\right)_{\mathrm{omb}}
$$

où les morphisme de transition de la limite projective sont induits par ceux du système projectif $\left(\mathfrak{J}_{n}^{0}\right)$. Soit

$$
{ }^{(m)} \Im_{\text {omb }}\left(\text { resp. }\left({ }^{(m)} \Im_{n}\right)_{\mathrm{omb}}\right)
$$

le $\mathfrak{J}$-torseur (resp. $\mathfrak{J}_{n}$-torseur) défini à partir du $\mathfrak{J}^{0}$-torseur (1.5.9) (resp. $\mathfrak{J}_{n}^{0}$ torseur (1.5.8)) et du $S$-homomorphisme $\mathfrak{J}^{0} \longrightarrow \mathfrak{I}$ (resp. $\mathfrak{I}_{n}^{0} \longrightarrow \mathfrak{I}_{n}$ ).

On a alors de manière évidente un système inductif

$$
\left({ }^{(m)} \Im_{\Im_{\mathrm{omb}}^{0}}^{0}\right)\left(\operatorname{resp} .\left({ }^{(m)} \Im_{\mathrm{omb}}\right),\left(\left({ }^{(m)} \Im_{n}^{0}\right)_{\mathrm{omb}}\right),\left(\left({ }^{(m)} \Im_{n}\right)_{\mathrm{omb}}\right)\right)(m>0)
$$

de $\mathfrak{J}^{0}\left(\right.$ resp. $\left.\mathfrak{I}, \mathfrak{J}_{n}^{0}, \mathfrak{J}_{n}\right)$-torseurs au dessus du système inductif $\left(\operatorname{omb}\left(W_{m}\right)\right)$.

Notons par

$$
\Im_{\mathrm{omb}}^{0}\left(\text { resp. } \Im_{\mathrm{omb}},\left(\Im_{n}^{0}\right)_{\mathrm{omb}},\left(\Im_{n}\right)_{\mathrm{omb}}\right)
$$

le $S$-faisceau limite inductive défini par ce système. On a ainsi un $\mathfrak{J}^{0}$ (resp. $\mathfrak{J}$, $\mathfrak{I}_{n}^{0}, \mathfrak{J}_{n}$ )-torseur de Zariski au-dessus de $\operatorname{omb}(\check{W})$.

On définit

$$
{ }^{(m)} \Im_{\mathrm{omb}}^{\underline{\nu}}\left(\text { resp. }\left({ }^{(m)} \Im_{n}^{\frac{\nu}{n}}\right)_{\mathrm{omb}}\right)
$$

à partir de l'augmentation induite par $\varepsilon$ (resp. $\varepsilon_{n}$ ) (cf. (0.26)). On définit de même $\Im_{0 \mathrm{mb}}^{\underline{v}}\left(\right.$ resp. $\left.\left(\Im_{n}^{\frac{v}{n}}\right)_{\mathrm{omb}}\right)$. On a alors

$$
\Im_{\mathrm{omb}}^{\underline{v}}=\lim _{\vec{m}}^{(m)} \Im_{\mathrm{omb}}^{\underline{v}}\left(\operatorname{resp} .\left(\Im_{n}^{\frac{v}{n}}\right)_{\mathrm{omb}}=\lim _{\vec{m}}\left({ }^{(m)} \Im_{n}^{\underline{v}}\right)_{\mathrm{omb}}\right)
$$

On donne une interprétation géométrique des objets ci-dessus calqués sur la construction de (1.1.18) dont on reprend les notations. Soient

$$
{ }^{(m)} L^{\underline{v}} \quad\left(\text { resp. }{ }^{(m)} L_{n}^{\underline{v}}\right)
$$

le module inversible sur $\mathfrak{X} \times_{S} \operatorname{omb}\left(W_{m}\right)$ (resp. $\mathfrak{X}_{n} \times_{S} \operatorname{omb}\left(W_{m}\right)$ ) donné par $\operatorname{Sym}^{(m)}\left(\mathcal{I}_{\Delta}\right)^{\underline{v}}\left(\right.$ resp. $\left.\operatorname{Sym}^{(m)}\left(\mathcal{I}_{\Delta}\right)^{\frac{v}{n}}\right)($ cf. $(0.55))$, et

$$
\left({ }^{(m)} L^{\underline{v}}\right)^{*}\left(\operatorname{resp} .\left({ }^{(m)} L_{n}^{\frac{v}{n}}\right)^{*}\right)
$$


le fibré principal associé. Le faisceau image directe

$$
\operatorname{pr}_{*}\left(\left({ }^{(m)} L^{\underline{v}}\right)^{*}\right)\left(\operatorname{resp} \cdot \operatorname{pr}_{*}^{n}\left(\left({ }^{(m)} L_{\bar{n}}^{\underline{v}}\right)^{*}\right)\right)
$$

au-dessus de $\operatorname{omb}\left(W_{m}\right)$ est un $\underline{\mathrm{G}}_{m}(\mathfrak{X})\left(\operatorname{resp} . \underline{\mathrm{G}}_{m}\left(\mathfrak{X}_{n}\right)\right)$-torseur canoniquement isomorphe à

$$
{ }^{(m)} \Im_{\mathrm{omb}}^{\underline{v}}\left(\operatorname{resp} .\left({ }^{(m)} \Im_{\bar{n}}^{\underline{v}}\right)_{\mathrm{omb}}\right) .
$$

On définit un système inductif de faisceaux inversibles

$$
\left({ }^{(m)} L^{\underline{v}}\right) \quad\left(\operatorname{resp} .\left({ }^{(m)} L_{\bar{n}}^{\underline{v}}\right)\right)
$$

au-dessus de $\left(\mathfrak{X} \times \operatorname{omb}\left(W_{m}\right)\right)\left(\right.$ resp. $\left.\left(\mathfrak{X}_{n} \times \mathrm{omb}\left(W_{m}\right)\right)\right)$. Par passage à la limite inductive on a donc un module inversible

$$
L^{\underline{\underline{v}}}\left(\operatorname{resp} . L_{\bar{n}}^{\underline{v}}\right)
$$

au-dessus de $\mathfrak{X} \times \operatorname{omb}(\check{W})$ (resp. $(\mathfrak{X} \times \operatorname{omb}(\check{W}))$ ). On désigne par $\left(L^{\underline{v}}\right)^{*}$ (resp. $\left.\left({ }^{(m)} L_{\bar{n}}^{\underline{v}}\right)^{*}\right)$ le fibré principal associé à $L^{\underline{v}}$ (resp. $\left.L_{\bar{n}}^{\underline{v}}\right)$. L'image directe $\operatorname{pr}_{*}\left(\left(L^{\underline{v}}\right)^{*}\right)\left(\right.$ resp. $\left.\operatorname{pr}_{*}^{n}\left(\left(L_{n}^{\underline{v}}\right)^{*}\right)\right)$ est un $\underline{G}_{m}(\mathfrak{X})\left(\operatorname{resp} . \underline{G}_{m}\left(\mathfrak{X}_{n}\right)\right)$-torseur audessus de omb $(\check{W})$ qui est canoniquement isomorphe à $\Im_{\text {omb }}^{\underline{v}}\left(\right.$ resp. $\left.\left(\Im_{n}^{\frac{\nu}{n}}\right)_{\text {omb }}\right)$. (Où pr : $\mathfrak{X} \times \operatorname{omb}(\check{W})\left(\right.$ resp. $\left.\operatorname{pr}_{n}: \mathfrak{X}_{n} \times \operatorname{omb}(\check{W})\right) \longrightarrow \operatorname{omb}(\check{W})$ désigne la projection.)

Le $\mathcal{O}_{\mathfrak{X} \times \mathfrak{x} \text {-module inversible }}$

$$
\operatorname{Sym}^{(1)}\left(\mathcal{I}_{\Delta}\right)^{1}=\mathcal{I}_{\Delta} \subset \mathcal{O}_{\mathfrak{X} \times \mathfrak{X}}
$$

donne lieu à un idéal $\mathcal{I}_{4}^{\mathrm{omb}} \operatorname{de} \mathrm{omb}(\mathfrak{X} \times \mathfrak{X})\left(\mathrm{cf}\right.$. (0.55)). On obtient ${ }^{(1)} L^{\underline{1}}$ (resp. (1) $\left.L_{\bar{n}}^{\frac{1}{n}}\right)$ comme la restriction de $\mathcal{I}_{4}^{\text {omb }}$ à $\mathfrak{X} \times \operatorname{omb}(\mathfrak{X})\left(\operatorname{resp} . \mathfrak{X}_{n} \times \operatorname{omb}(\mathfrak{X})\right)$.

On vérifie qu'un générateur de $\mathcal{I}_{\Delta}$ donne lieu à un générateur de $\mathcal{I}_{\Delta}^{\text {omb }}$ dont la restriction à $\mathfrak{X} \times \mathfrak{U} \hookrightarrow \mathfrak{X} \times \operatorname{omb}(\mathfrak{X})$ est inversible, donc

$$
\underline{1} \in \Gamma\left(\mathfrak{X} \times \mathfrak{U},{ }^{(1)} L^{\underline{1}}\right) \quad\left(\operatorname{resp} . \underline{1} \in \Gamma\left(\mathfrak{X}_{n} \times \mathfrak{U},{ }^{(1)} L_{\bar{n}}^{1}\right)\right) .
$$

La section 1 donne lieu alors à une section

$$
f^{\prime} \text { (resp. } f_{n}^{\prime} \text { ) de } \operatorname{pr}_{*}\left({ }^{(1)} L^{-1}\right)^{*} \simeq{ }^{(1)} \Im_{\text {omb }}^{-1}\left(\text { resp. } \operatorname{pr}_{*}^{n}\left({ }^{(1)} L_{\bar{n}}^{\frac{1}{n}}\right)^{*} \simeq\left({ }^{(1)} \Im_{n}^{1}\right)_{\text {omb }}\right) \text {. }
$$

Il est clair que $f_{n}^{\prime}$ est égale au composé de $f^{\prime}$ avec ${ }^{(1)} \Im_{\text {omb }}^{-1} \longrightarrow\left({ }^{(1)} \Im_{n}^{\frac{1}{n}}\right)_{\text {omb }}$.

DÉFINITION 1.5.18. On désigne par

$$
f: \mathfrak{U} \longrightarrow \Im_{\text {omb }}
$$

le S-morphisme obtenu en composant $f^{\prime}$ avec ${ }^{(1)} \Im_{o m b}^{\frac{1}{o}} \longrightarrow \Im_{o m b}$, et on l'ap- 
pelle le morphisme d'Abel-Jacobi donné par le couple ( $\mathfrak{X}, \Im)$. On note par $f_{n}: \mathfrak{U} \longrightarrow\left(\Im_{n}\right)_{\text {omb }}$ le composé de $f$ (resp. $\left.f_{n}^{\prime}\right)$ avec $\Im_{\text {omb }} \longrightarrow\left(\Im_{n}\right)_{\text {omb }}$ (resp. $\left.\left({ }^{(1)} \Im_{n}^{\frac{1}{n}}\right)_{\text {omb }} \longrightarrow\left(\Im_{n}\right)_{o m b}\right)$.

On donne la description suivante du morphisme d'Abel-Jacobi $f$ en termes du choix d'un générateur $T$ de $I$. On a alors le relèvement suivant du morphisme $\gamma^{0}$

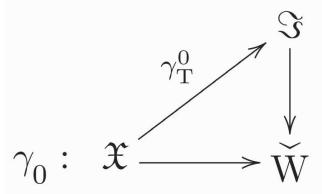

$$
\text { (cf. }(0.35),(\text { ii) ). }
$$

Par définition du $\mathfrak{I}$-torseur ${ }^{(1)} \Im$ on a alors un isomorphisme ${ }^{(1)} \Im \simeq \mathfrak{X} \times \mathfrak{J}$ de $\mathfrak{J}$-torseurs au-dessus de $\mathfrak{X}$ qui donne lieu à un isomorphisme de $\mathfrak{J}$-torseurs

$$
{ }^{(1)} \Im_{\mathrm{omb}} \stackrel{\sim}{\longrightarrow} \operatorname{omb}(\mathfrak{X}) \times \mathfrak{J}
$$

par définition de ${ }^{(1)} \Im_{o m b}$ (cf. (1.5.7)). Le morphisme d'Abel-Jacobi $f \in \Gamma\left(\mathfrak{U},{ }^{(1)} \Im_{\text {omb }}\right)$ corrrespond par la bijection suivante induite par (1.5.19)

$$
\Gamma\left(\mathfrak{H},{ }^{(1)} \Im_{\mathrm{omb}}\right) \stackrel{\sim}{\longrightarrow} \mathfrak{I}(\mathfrak{H})
$$

à la section $-\varphi_{T}^{1}=-T(t-T)^{-1}$. On voit donc que $f$ se définit à l'aide du morphisme $\gamma_{T}^{0}: \mathfrak{X} \longrightarrow \breve{W}$ et du morphisme $\varphi_{T}^{1}: \mathfrak{U} \longrightarrow \mathfrak{J}$, le premier vérifie la propriété universelle donnée par (0.39) et le second celle donnée par (1.4.4). Il en résulte que $f: \mathfrak{U} \longrightarrow \Im_{0 m b}$ vérifie aussi une propriété universelle que l'on verra dans le prochain paragraphe.

(1.6) On désigne par $G$ un $S$-schéma en groupes commutatif et lisse. Soit $G^{0}$ la composante neutre de $G$. On a alors que $G^{0}$ est représentable par un ouvert de $G$, séparé et de présentation finie au-dessus de $S$ ([7 bis], $\mathrm{VI}_{B}, 3.10$ et 5.5).

Soit $U$ un générateur de $I$. On lui associe alors un scindage

$$
{ }^{(m)} \sigma_{U}: W_{m} \longrightarrow{ }^{(m)} \Im(\text { cf. }(0.47))\left(\text { resp. } \sigma_{U}: \breve{W} \longrightarrow \Im\right),
$$

du $\mathfrak{J}_{\text {-torseur }}{ }^{(m)} \Im \longrightarrow W_{m}$ (resp. de l'extension $1 \longrightarrow \mathfrak{J} \longrightarrow \Im \longrightarrow \breve{W} \longrightarrow 1$ ), qui donne lieu, par définition de ${ }^{(m)} \Im_{o m b}$, à un scindage

$$
{ }^{(m)} \sigma_{U \text { omb }}: \operatorname{omb}\left(W_{m}\right) \longrightarrow{ }^{(m)} \Im_{\text {omb }},
$$

d'où un scindage de $\Im_{\text {omb }}$ donné par le système inductif $\left({ }^{(m)} \sigma_{U \text { omb }}\right)$

$$
\sigma_{U \mathrm{omb}}: \operatorname{omb}(\check{W}) \longrightarrow \Im_{\mathrm{omb}}
$$


Soit

$$
h: \Im \longrightarrow G
$$

un $S$-homomorphisme. Soit $h^{\prime}: \mathfrak{J} \longrightarrow G$ l'homomorphisme obtenu par restriction de $h$ à $\mathfrak{J}$. D'autre part le morphisme

$$
h \circ \sigma_{U}: \check{W} \longrightarrow G
$$

se factorise par $G^{0} \longrightarrow G$. On peut alors appliquer (1.5.5) et on trouve ainsi un $S$-morphisme $\left(h \circ \sigma_{U}\right)_{\text {omb }}: \operatorname{omb}(\check{W}) \longrightarrow G$, tel qui redonne $h \circ \sigma_{U}$ par restriction à $\check{W} \longrightarrow \operatorname{omb}(\check{W})$. Il existe alors un unique morphisme

$$
h_{\mathrm{omb}}: \Im_{\mathrm{omb}} \longrightarrow G
$$

qui rend commutatif le diagramme suivant

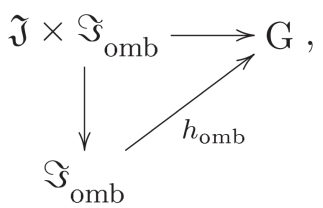

où la flèche horizontale est obtenue à partir de $h^{\prime}, h_{\mathrm{omb}}$, et la loi de composition de $G$. On vérifie que $h_{\text {omb }}$ est indépendant du choix de $U$. On a alors una application

$$
\operatorname{Hom}_{S-g r}(\Im, G) \longrightarrow G(\mathfrak{H})
$$

qui fait correspondre à un $S$-homomorphisme $h: \Im \longrightarrow G$ la section $h_{\text {omb }} \circ f$ de $G$ au-dessus de $\mathfrak{U}$. On peut maintenant énoncer le théorème principal de ce travail.

THÉORÈME 1.6.6. Soit $S=\operatorname{Spec}(A)$ un schéma affine et noethérien. Soit $G$ un $S$-schéma en groupes commutatif, lisse et séparé. Soient $B$ une A-algèbre adique isomorphe à $A[[T]], \mathfrak{X}=S p f(B), \mathfrak{U}$ et $\Im$ définis à partir de $\mathfrak{X}$ comme dans $\S 0$. On a alors que pour toute section $\sigma \in G(\mathfrak{U})$ il existe un unique S-homomorphisme

$$
h: \Im \longrightarrow G
$$

tel que $\sigma=h_{\text {omb }} \circ f$, où $f: \mathfrak{U} \longrightarrow \Im_{\text {omb }}$ désigne le morphisme d'Abel-Jacobi

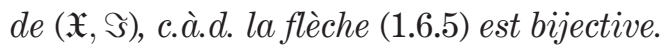




\section{Montrons d'abord le}

LEMME 1.6.7. Sous les hypothèses de (1.6.6) on a que la flèche (1.6.5) est injective.

Preuve. Soient $h, g \in \operatorname{Hom}_{S-g r}(\Im, G)$ tels que $h_{\text {omb }} \circ f=g_{\text {omb }} \circ f$, montrons que $h=g$. Soient $h^{\prime}, g^{\prime}$ les restrictions de $h$ et de $g \grave{a} \mathfrak{J}$.

Puisque le morphisme $h_{\text {omb }}$ (resp. $g_{\text {omb }}$ ) rend commutatif le diagramme (1.6.4), on a

$$
h^{\prime} \circ\left(-\varphi_{T}^{1}\right) \equiv g^{\prime} \circ\left(-\varphi_{T}^{1}\right)\left(\bmod G(\mathfrak{X})^{+}\right) .
$$

Soit $\beta: \mathfrak{I} \longrightarrow \mathfrak{J}$ l'automorphisme définit dans (1.3.28). On a alors $\beta\left(-\varphi_{T}^{1}\right)=\varphi_{T}^{1}$, d'où

$$
\left(h^{\prime} \circ \beta^{-1}\right) \circ \varphi_{T}^{1} \equiv\left(g^{\prime} \circ \beta^{-1}\right) \circ \varphi_{T}^{1}\left(\bmod G(\mathfrak{X})^{+}\right) .
$$

On a alors d'après (1.4.5) tel que $h^{\prime} \circ \beta^{-1}=g^{\prime} \circ \beta^{-1}$, d'où que $h^{\prime}=g^{\prime}$. Donc

$$
\left(h \circ \gamma_{T}^{0}\right)_{o m b}^{\prime}=\left(g \circ \gamma_{T}^{0}\right)_{o m b}^{\prime}
$$

où $\left(h \circ \gamma_{T}^{0}\right)_{\text {omb }}^{\prime}\left(r e s p .\left(g \circ \gamma_{T}^{0}\right)_{o m b}^{\prime}\right)$ désigne la restriction de $\left(h \circ \gamma_{T}^{0}\right)_{\text {omb }}$ (resp. $\left.\left(g \circ \gamma_{T}^{0}\right)_{\text {omb }}\right) \grave{a} \mathfrak{U} \subset$ omb( $\left.\mathfrak{X}\right)$. On a alors

$$
\left(h \circ \gamma_{T}^{0}\right)_{o m b}=\left(g \circ \gamma_{T}^{0}\right)_{o m b}
$$

car $G^{0}$ est séparé. On conclut que les restrictions $h^{\prime \prime}$ et $g^{\prime \prime}$ respectivement de $h$ et $g \grave{a} \sigma_{T}(\breve{W})(c f .(0.46))$ coïncident (cf. (0.39)). Il en résulte que $h=g$, car $h=h^{\prime} h^{\prime \prime}$ et $g=g^{\prime} g^{\prime \prime}$.

Réduction de la preuve de (1.6.6) à celle de (1.4.4).

D’après (1.6.7) il suffit de montrer que la flèche (1.6.5) est surjective.

Soit donc $\sigma \in G(\mathfrak{H})$. D'après (1.4.4) il existe un unique S-homomorphisme

$$
h^{\prime}: \mathfrak{J} \longrightarrow G
$$

tel que $\bar{\sigma}=\sigma\left(h^{\prime} \circ \varphi_{T}^{1}\right)^{-1} \in G(\mathfrak{X})^{+}$. Notons que $\tilde{\sigma}$ la restriction de $\bar{\sigma} \grave{\alpha} \mathfrak{X}$.

On a alors $\tilde{\sigma}_{\text {omb }}=\bar{\sigma}$ car $G$ est séparé. D'après (0.39) on peut trouver un S-homomorphisme $h^{\prime \prime}: \sigma_{T}(\check{W}) \longrightarrow G$ tel que $h^{\prime \prime} \circ \gamma_{T}^{0}=\tilde{\sigma}$, d'où

$$
\left(h^{\prime \prime} \circ \gamma_{T}^{0}\right)_{o m b}=\tilde{\sigma}_{o m b}=\bar{\sigma} \text {. }
$$

Soit $h: \Im \longrightarrow G$ le S-homomorphisme tel que $h \mid \mathfrak{I}=h^{\prime}$, et $\left.h\right|_{\sigma_{T}(\check{W})}=h^{\prime \prime}$. Par définition de (1.6.5) on a que $h_{\text {omb }} \circ f=\sigma$. 
(1.7) Soient $X, D,(J, \Phi), \ldots$ etc. comme dans (1.2).

Supposons que $S$ est noethérien et que $D$ soit donné par une section de $X$ au-dessus de $S$, et que le complété formel $\mathfrak{X}=\widehat{X}_{/ D}$ de $X$ le long de $D$ soit isomorphe à $\operatorname{Spf}(A[[T]])$. Soient $\Im, f$ (morphisme d'Abel-Jacobi de $(\Im, \mathfrak{X}))$,... etc. définis à partir de $\mathfrak{X}$ comme dans $\S 0$. On veut expliciter les rapports qui existent entre $(\Im, f)$ et $(J, \Phi)$.

Le morphisme (1.2.8) donne lieu par passage à la limite projective à un S-homomorphisme

$$
\Im \simeq \lim _{\overleftarrow{n}} \underline{\mathcal{P i c}}_{\mathfrak{x}^{(n)} / S} \longrightarrow J=\lim _{\overleftarrow{n}} \underline{\operatorname{Pic}}_{X^{(n)} / S}
$$

qui donne lieu à partir de la construction de (1.6.3) à un S-homomorphisme

$$
\Im_{\mathrm{omb}} \longrightarrow J
$$

que l'on se propose d'interpréter géométriquement. Soit $L_{\Delta}$ l'idéal de définition de la diagonale $\Delta \subset X \times_{S} X$. Soit $\operatorname{Sym}^{(m)}(X)$ la puissance symétrique $m$-ème de $X$. Pour toute section $\underline{v}$ de $\underline{Z}_{S}$ on définit le $\mathcal{O}_{X{ }_{S} \operatorname{Sym}^{(m)}(X)^{-}}$ module inversible $\operatorname{Sym}^{(m)}\left(L_{\Delta}\right)^{\underline{v}}$ en suivant la définition de $\operatorname{Sym}^{(m)}\left(\mathcal{I}_{\Delta}\right)^{\underline{v}}$. Le morphisme (1.7.1) est caractérisé par le fait que, l'image du couple $\left(\operatorname{Sym}^{(m)}\left(\mathcal{I}_{\Delta}\right)^{\underline{v}}, \xi\right)$, formé par le $\mathcal{O}_{\mathfrak{x} \times{ }_{S} W_{m}}$-module inversible $\operatorname{Sym}^{(m)}\left(\mathcal{I}_{\Delta}\right)^{\underline{v}}$, et d'une trivialisation $\xi$ de $\operatorname{Sym}^{(m)}\left(\mathcal{I}_{\Delta}\right)$ le long de $\mathfrak{X} \times_{S} W_{m}$, est égale à la classe du couple $\left(\operatorname{Sym}^{(m)}\left(L_{\Delta}\right)_{W_{m}}^{\frac{v}{}}, \bar{\xi}\right)$, où $\operatorname{Sym}^{(m)}\left(L_{\Delta}\right)_{W_{m}}^{\frac{v}{}}$ désigne la restriction de $\operatorname{Sym}^{(m)}\left(L_{\Delta}\right)^{\underline{v}}$ à $X \times_{S} W_{m}$, et $\bar{\xi}$ désigne la trivialisation de $\operatorname{Sym}^{(m)}\left(L_{\Delta}\right)_{W_{m}}^{\frac{v}{}}$ donnée par $\xi$.

Notons par $\operatorname{Sym}^{(m)}\left(L_{\Delta}\right)_{\mathrm{omb}\left(W_{m}\right)}^{\underline{v}}$ la restriction de $\operatorname{Sym}^{(m)}\left(L_{\Delta}\right)^{\underline{v}}$ à $X \times_{S} \operatorname{omb}\left(W_{m}\right)$. On a alors que (1.7.2) fait correspondre au couple $\left({ }^{(m)} L^{\underline{v}}, \xi\right)$ où $\xi$ désigne une trivialisation de ${ }^{(m)} L^{\underline{v}}$ le long de $\mathfrak{X} \times_{S}$ omb $\left(W_{m}\right)$, la classe du couple $\left(\operatorname{Sym}^{(m)}\left(L_{\Delta}\right)_{\mathrm{omb}\left(W_{m}\right)}^{\underline{v}}, \bar{\xi}\right)$, où $\bar{\xi}$ désigne la trivialisation donnée par $\xi$.

Par définition du morphisme d'Abel-Jacobi $f: \mathfrak{U} \longrightarrow \Im_{\text {omb }}$ correspond, d'après (1.5.18), le couple $\left(\mathcal{I}_{\Delta}^{\mathrm{omb}}, \underline{1}\right)$, où $\underline{1}$ désigne la trivialisation de $\mathcal{I}_{\Delta}^{\mathrm{omb}}$ audessus de $\mathfrak{X} \times_{S} \mathfrak{U}$ donnée par $\underline{1}$. On a alors par ce qui précède que l'image de $f$ par (1.7.2) coïncide avec la classe du couple $\left(\left(L_{\Delta}\right)_{\mathfrak{U}}, \underline{1}_{\mathfrak{l}}\right)$ que l'on note $\Phi_{\mathfrak{U}}$ dans (1.2.13). On a ainsi le 
LEMME 1.7.3. Le diagramma suivant est commutatif

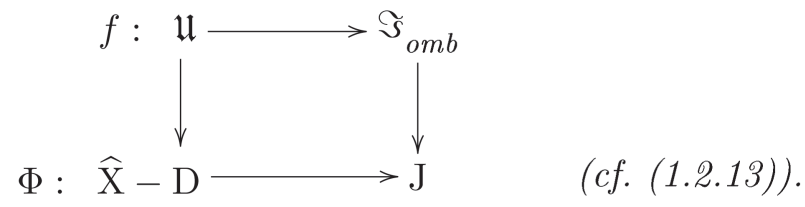

\section{Preuve de (1.4.4) dans le cas d'un corps de base}

Le (1.4.6) permet de ramener la preuve de (1.4.4) pour un corps de base $k$ au cas où celui-ci est algébriquement clos. Dans ce numéro on désigne par $k$ un corps algébriquement clos.

Soit $G$ un $k$-schéma en groupes commutatif et lisse. On a alors une suite exacte

$$
1 \longrightarrow G^{0} \longrightarrow G \longrightarrow A \longrightarrow 1,
$$

où $G^{0}$ est le produit d'un $k$-tore trivial $T$ par un $k$-groupe lisse unipotent commutatif $N$, et $A$ est une extension d'un $k$-groupe étale $\Gamma$ par une $k$ variété abélienne $A^{0}$ (cf. [15]).

Soit $B=k[[T]]$. Soient $\mathfrak{J}, \mathfrak{J}^{0}, \varphi_{T}^{1}, \varphi_{T}^{0} \ldots$ etc construits à partir de $B$ comme dans $\S 0$.

$\underline{\text { Il est facile de vérifier que } \rho^{+} \text {est une bijection si et seulement si } \rho \text { en est }}$ une. Montrons donc que

$$
\rho: \operatorname{Hom}_{\mathrm{k}-\mathrm{gr}}\left(\mathfrak{J}^{0}, G\right) \stackrel{\sim}{\longrightarrow} F(G)
$$

défini par composition avec $\varphi_{T}^{0}$, est bijective. On a le diagramme d'homomorphismes de groupes à lignes exactes :

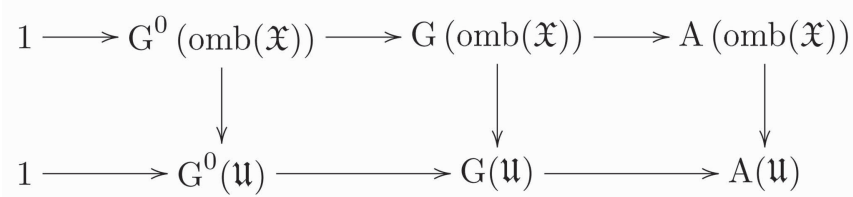

Soit $g \in G(\mathfrak{U})$. L'image de $\bar{g}$ de $g$ dans $A(\mathfrak{U})$ provient d'une section, que l'on note aussi $\bar{g}$, de $A(\operatorname{omb}(\mathfrak{X}))$ par la restriction $A(\operatorname{omb}(\mathfrak{X})) \longrightarrow A(\mathfrak{H})$, en vertu du critère valuatif de propreté. (Noter que $B^{*}=k[[T]]\left[T^{-1}\right]$ est alors un corps valué, et que les composantes connexes de $A$ sont des $k$-schémas propres). Puisque le $G^{0}$-torseur $G$, au-dessus de $A$ est Zariskien, il existe 
un relèvement $g^{\prime} \in G(\operatorname{omb}(\mathfrak{X}))$ de $\bar{g} \in A(\operatorname{omb}(\mathfrak{X}))$. (Remarquer que $\operatorname{omb}(\mathfrak{X})=\operatorname{Spec}(k[[T]])$ est un $k$-schéma local). On a alors

$$
g g^{\prime-1} \in G^{0}(\mathfrak{U}),
$$

et il en résulte que

$$
F\left(G^{0}\right)=F(G)
$$

D'autre part

$$
\operatorname{Hom}_{\mathrm{k}-\mathrm{gr}}\left(\mathfrak{J}^{0}, A\right)=0
$$

car il n'y a pas de $k$-homomorphisme non-trivial d'un $k$-groupe affine connexe dans la $k$-variété abélienne $A^{0}$, ni dans le $k$-groupe étale $\Gamma$. La suite exacte (2.1) donne alors lieu à un isomorphisme de groupes

$$
\operatorname{Hom}_{\mathrm{k}-\mathrm{gr}}\left(\mathfrak{J}^{0}, G^{0}\right) \simeq \operatorname{Hom}_{\mathrm{k}-\mathrm{gr}}\left(\mathfrak{J}^{0}, G\right) .
$$

On est alors ramené à montrer que $\varphi_{T}^{0}$ induit une bijection $\operatorname{Hom}_{\mathrm{k}-\mathrm{gr}}\left(\mathfrak{J}^{0}, G^{0}\right) \simeq F\left(G^{0}\right)$. Noter que $F\left(G^{0}\right)=F(T) \times F(N)$. Il suffit alors de montrer que $\rho$ est bijective pour $G=T$ et pour $G=N$.

Comme le corps $k$ est algébriquement clos on a que $T$ est un produit d'un nombre fini de copies de $\underline{\mathrm{G}}_{\mathrm{mk}}$. Il suffit alors de montrer que $\rho$ est bijective si $T=\underline{\mathrm{G}}_{\mathrm{mk}}$. Compte tenu que $\mathfrak{J}^{0}=\underline{\mathrm{G}}_{\mathrm{mk}} \times \wedge^{0}$, que $\wedge^{0}$ est un $k$ groupe pro-unipotent, et qu'il n'y a pas de $k$-homomorphisme non-trivial d'un $k$-groupe unipotent $N$ dans $\underline{G}_{\mathrm{mk}}$ (cf. [6]), la flèche

$$
\operatorname{Hom}_{\mathrm{k}-\mathrm{gr}}\left(\underline{\mathrm{G}}_{\mathrm{mk}}, \underline{\mathrm{G}}_{\mathrm{mk}}\right) \longrightarrow \operatorname{Hom}_{\mathrm{k}-\mathrm{gr}}\left(\mathfrak{J}^{0}, \underline{\mathrm{G}}_{\mathrm{mk}}\right)
$$

induite par la projection $\mathfrak{J}^{0} \longrightarrow \underline{G}_{m k}$ est bijective. On écrit

$$
\varphi_{T}^{0}=t\left(1-t^{-1} T\right),
$$

avec $t \in \underline{\mathrm{G}}_{\mathrm{mk}}\left(k[[t]]\left[t^{-1}\right]\right)$. On a alors $\left(1-t^{-1} T\right) \in \wedge^{0}\left(k[[t]]\left[t^{-1}\right]\right)$. Soit $h \in \operatorname{Hom}_{\mathrm{k}-\mathrm{gr}}\left(\underline{\mathrm{G}}_{\mathrm{mk}}, \underline{\mathrm{G}}_{\mathrm{mk}}\right) \simeq \mathrm{Z}$ donné par $x \longrightarrow x^{n}, n \in \mathbb{Z}$. On a alors que $\rho$ associe à $h$ la classe de $t^{n}$ dans

$$
F\left(\underline{\mathrm{G}}_{\mathrm{mk}}\right)=\underline{\mathrm{G}}_{\mathrm{mk}}\left(k[[t]]\left[t^{-1}\right]\right) / \underline{\mathrm{G}}_{\mathrm{mk}}[[t]] .
$$

Or ce dernier est le groupe de la valuation de $k[[t]]\left[t^{-1}\right]$, qui est isomorphe à $Z$, et on a alors $\rho(h)=n$. Ce qui donne que $\rho$ est bijective.

Soit $G$ un $k$-groupe lisse unipotent commutatif. Il existe alors une suite de composition

$$
0=G_{n} \subset \ldots \subset G_{1} \subset G_{0}=G
$$


tel que pour tout $1 \leq i \leq n-1$ le groupe $G_{i} / G_{i+1}$ soit isomorphe au groupe additif $\underline{G}_{\text {ak }}$ pour tout $0 \leq i \leq n-1$ (cf. [6]). On procède à montrer que $\rho$ est bijective par récurrence sur la dimension $n$ de $G$.

Montrons d'abord que

$$
\rho: \operatorname{Hom}_{\mathrm{k}-\mathrm{gr}}\left(\mathfrak{J}^{0}, \underline{\mathrm{G}}_{\mathrm{ak}}\right) \longrightarrow F\left(\underline{\mathrm{G}}_{\mathrm{ak}}\right)
$$

est une bijection. Notons que

$$
\operatorname{Hom}_{\mathrm{k}-\mathrm{gr}}\left(\mathfrak{J}^{0}, \underline{\mathrm{G}}_{\mathrm{ak}}\right)=\operatorname{Hom}_{\mathrm{k}-\mathrm{gr}}\left(\wedge^{0}, \underline{\mathrm{G}}_{\mathrm{ak}}\right)
$$

car il n'y a pas de $k$-homomorphisme non-trivial de $\underline{G}_{m k}$ dans $\underline{G}_{\mathrm{ak}}$. Étant donné une section $F(T) \in \underline{G}_{\mathrm{ak}}\left(k[[T]]\left[T^{-1}\right]\right)$ on lui associe un $k$-homomorphisme

$$
h_{F(T)}: \wedge^{0} \longrightarrow \underline{\mathrm{G}} \mathrm{ak}
$$

défini par

$$
h_{F(T)}(u)=\operatorname{Res}\left(F(T) \frac{d u}{u}\right)
$$

et $u \in \wedge^{0}(A)$ où $A$ désigne une $k$-algèbre. On définit $\operatorname{Res}\left(F(T) \frac{d u}{u}\right)$ comme dans [16]. La fonctorialité de l'opération Res montre que $h_{F(T)}$ est un morphisme de $k$-groupes (cf. loc. cit.).

En vertu de (1.4.5) il suffit de prouver que $\rho$ est surjectif. On a la formule suivante

$$
\operatorname{Res}\left(F(T) \frac{d \varphi^{0}}{\varphi^{0}}\right) \equiv-F(t) \bmod \left(\underline{\mathrm{G}}_{\mathrm{ak}}(k[[T]])\right),
$$

En effet, pour tout entier positif $m$ on a l'identité suivante dans $\left(k[[t]]\left[t^{-1}\right]\right)[[T]]\left[T^{-1}\right] d T$ :

$$
T^{-m} \frac{d\left(1-t^{-1} T\right)}{1-t^{-1} T}=-\sum_{0 \leq i} \frac{T^{-m+i}}{t^{i+1}} d T,
$$

d'où il résulte que le coefficient de $\frac{d T}{T}$ est égal à $-\frac{1}{t^{m}}$, ce qui montre (2.14).

La suite de composition (2.9) donne pour tout $0 \leq i<n$ la suite exacte suivante

$$
1 \longrightarrow G_{i} / G_{i+1} \longrightarrow G / G_{i+1} \longrightarrow G / G_{i} \longrightarrow 1 .
$$

Pour achever la réccurence il suffit de voir qu'étant donné une suite exacte de $k$-groupe unipotents et lisses

$$
1 \longrightarrow \underline{G}_{\mathrm{ak}} \longrightarrow G \longrightarrow G^{\prime} \longrightarrow 1
$$


tel que $\rho: \operatorname{Hom}_{\mathrm{k}-\mathrm{gr}}\left(\wedge^{0}, G^{\prime}\right) \longrightarrow F\left(G^{\prime}\right)$ soit une bijection, on a alors que $\rho: \operatorname{Hom}_{\mathrm{k}-\mathrm{gr}}\left(\wedge^{0}, G\right) \longrightarrow F(G)$ est aussi une bijection.

Rappelons d'abord quelques résultats. Soit $k$ un corps parfait de caractéristique $p>0$. On désigne par $W(k)^{+}$le groupe additif des vecteurs de Witt sur $k$, et par $W_{r}(k)^{+}$son $r$-ème tronqué, vus comme $k$-foncteurs en groupes. On a alors :

(a) Le $k$-groupe $\wedge^{0}$ est isomorphe à un produit d'une infinité de copies de $W(k)^{+}$, une copie pour chaque entier $q$ premier avec $p$. (Si $k$ est de caractéristique 0 alors $\wedge^{0}$ est isomorphe à un produit d'une infinité de copies de $\left.\underline{G}_{\mathrm{ak}}\right)$.

Soit

$$
F(t)=\prod_{(n, p)=1}\left(1-t^{n}\right)^{\mu(n) / n},
$$

où $\mu$ désigne la fonction de Möbius. Soit $A$ une $k$-algèbre. Soient $h \in T A[[T]]$ et

$$
\vec{x}=\left(x_{0}, x_{1}, \ldots\right)
$$

un vecteur de Witt à coefficients dans $A$, c.à.d. $\vec{x} \in W(k)^{+}(A)$. On pose

$$
\vec{h}=(h, 0, \ldots) \in W(k)^{+}(A[[T]]) \text {. }
$$

On a alors

$$
\vec{x} \cdot \vec{h}=\left(x_{0}, x_{1}, \ldots\right) \cdot(h, 0, \ldots)=\left(x_{0} h, x_{1} h^{p}, \ldots, x_{n} h^{p^{n}}, \ldots\right) .
$$

Soit

$$
\begin{aligned}
& E(\vec{x} \cdot \vec{h})=F\left(x_{0} h\right) F\left(x_{1} h^{p}\right) \ldots F\left(x_{n} h^{p^{n}}\right) \ldots \\
& \text { (Exponentielle de Artin-Hasse de } \vec{x} \cdot \vec{h}) .
\end{aligned}
$$

Etant donnée une section $\left(\vec{a}_{i}\right)$ du $k$-groupe $\left(W(k)^{+}\right)^{(i)}$ à valeurs dans la $k$-algèbre $A$, on lui associe la série formelle $(i, p)=1$

$$
g=\prod_{(i, p)=1} E\left(\vec{a}_{i} \vec{T}^{i}\right)
$$

de $A[[T]]$. On vérifie que l'on a ainsi un $k$-isomorphisme de groupes

$$
\prod_{(i, p)=1}\left(W(k)^{+}\right)^{(i)} \stackrel{\sim}{\longrightarrow} \wedge^{0}
$$

(cf. [13], pg. 102). Il en résulte que pour tout entier $n>1$ on a un $k$-iso- 
morphisme de groupes

$$
\prod_{(i, p)=1, i \leq n}\left(W_{r(i)}(k)^{+}\right)^{(i)} \stackrel{\sim}{\longrightarrow} \wedge_{n}^{0}
$$

où $r(i)$ dénote un entier dépendant de $i$ (cf. loc. cit., pg. 103, Proposition 9).

Dans le cas où $k$ est un corps de caractéristique 0 on a un $k$-isomorphisme

$$
\prod_{1 \leq i}\left(\underline{G}_{\mathrm{ak}}\right)^{(i)} \stackrel{\sim}{\longrightarrow} \wedge^{0}
$$

faisant correspondre à la section $\left(a_{i}\right)$ à valeurs dans une $k$-algèbre $A$ la série formelle

$$
g=\exp \left(\sum_{1 \leq i \leq n} \frac{a_{i} t^{i}}{i}\right)
$$

Pour tout entier $n \geq 1$ on a un $k$-isomorphisme

$$
\prod_{1 \leq i \leq n}\left(\underline{G}_{\mathrm{ak}}\right)^{(i)} \stackrel{\sim}{\longrightarrow} \wedge_{n}^{0}
$$

REMARQUE 2.26. bis(a) Dans le cas où k est de caractéristique 0 et u est une section $\wedge^{0}$ à valeur dans la k-algèbre A tel que $u=\exp \left(\sum_{1 \leq i} \frac{a_{i} t^{i}}{i}\right)$. On
a pour tout $i \geq 1$

$$
a_{i}=\operatorname{Res}\left(T^{-i} d u / u\right)
$$

Si $k$ est de caractéristique $p>0$, on introduit pour tout $i \geq 1$ premier avec $p$ le vecteur de Witt $\vec{T}^{-i}=\left(T^{-i}, 0, \ldots\right)$ à valeurs dans $k[[T]]\left[T^{-1}\right]$. Étant donné une section $u$ de $\wedge^{0}$ à valeurs dans une $k$-algèbre $A$ de la forme $u=\prod_{(i, p)=1} E\left(\vec{a}_{i} \cdot \vec{T}^{i}\right)$, soit $\operatorname{Res}\left(\vec{T}^{-i} d u / u\right)$ le symbole défini par Witt (cf. [17], pg. 369). On a alors pour tout entier $i>0$ premier avec $p$

$$
\vec{a}_{i}=\operatorname{Res}\left(\vec{T}^{-i} d u / u\right)
$$

(b) Si $k$ est un corps parfait de caractéristique $p>0$ on a

$$
\operatorname{Ext}_{k}^{1}\left(W(k)^{+}, \underline{G}_{\mathrm{ak}}\right)=0
$$

Si $k$ est de caractéristique 0 on a

$$
\operatorname{Ext}_{k}^{1}\left(\underline{G}_{\mathrm{ak}}, \underline{G}_{\mathrm{ak}}\right)=0 .
$$


On note par ${ }^{u} \varphi^{0}: \mathfrak{U} \longrightarrow \wedge^{0}$ le $k$-morphisme donné par la section $1-t^{-1} T \in \wedge^{0}\left(k[[t]]\left[t^{-1}\right]\right)$, et $\operatorname{par}^{u} \varphi_{n}^{0}: \mathfrak{U} \longrightarrow \wedge_{n}^{0}$ le composé de ${ }^{u} \varphi^{0}$ avec $\wedge^{0} \longrightarrow \wedge_{n}^{0}$.

Soit $g: \mathfrak{U} \longrightarrow G$ un $k$-morphisme. Par hypothèse (de récurrence) il existe un $k$-homomorphisme $h: \wedge_{n}^{0} \longrightarrow G^{\prime}$ tel que

$$
s^{\prime}=(\pi \circ g)\left(h \circ{ }^{u} \varphi_{n}^{0}\right)^{-1} \in G^{\prime}(\operatorname{omb}(\mathfrak{X})) .
$$

Comme le $\underline{G}_{\mathrm{ak}}$-torseur $G$ au-dessus de $G^{\prime}$ est de Zariski, on peut relever $s^{\prime}$

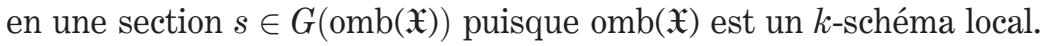
Quitte à substituer $g$ par $g s^{-1}$ on peut supposer que $g$ vérifie :

$$
\pi \circ g=h \circ{ }^{u} \varphi_{n}^{0} .
$$

On a alors le diagramme commutatif de $k$-morphismes :

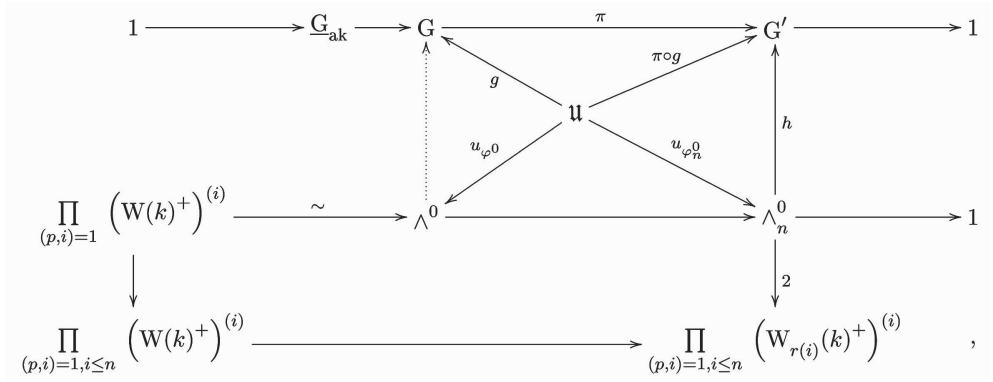

où la flèche en pointillé désigne le $k$-homomorphisme à construire. Soit

$$
0 \longrightarrow \underline{G}_{\mathrm{ak}} \longrightarrow H \longrightarrow \prod_{(p, i)=1, i \leq n}\left(W(k)^{+}\right)^{(i)} \longrightarrow 0
$$

la suite exacte de $k$-groupes obtenue à partir de (2.17) et du $k$-homomorphisme composé

$$
\prod_{(p, i)=1, i \leq n}\left(W(k)^{+}\right)^{(i)} \longrightarrow \wedge_{n}^{0} \stackrel{h}{\longrightarrow} G^{\prime}
$$

obtenu à partir de (2.30). Il résulte de (2.27) que

$$
\operatorname{Ext}_{k}^{1}\left(\prod_{(p, i)=1, i \leq n}\left(W(k)^{+}\right)^{(i)}, \underline{G}_{\mathrm{ak}}\right)=0,
$$

d'où l'extension (2.31) est triviale. Compte tenu que le carré inférieur du diagramme (2.30) commute on a alors qu'il existe un $k$-homomorphisme

$$
\bar{f}: \wedge^{0} \longrightarrow G
$$


qui relève $\wedge^{0} \longrightarrow \wedge_{n}^{0} \stackrel{h}{\longrightarrow} G^{\prime}$. On en déduit

$$
g\left(\bar{f} \circ^{u} \varphi^{0}\right)^{-1} \in \underline{G}_{\mathrm{ak}}(\mathfrak{H}) .
$$

On conclut qu'il existe un $k$-homomorphisme $f: \wedge^{0} \longrightarrow \underline{G}_{\mathrm{ak}}$ tel que

$$
g\left(\bar{f} \circ^{u} \varphi^{0}\right)^{-1} \equiv f \circ^{u} \varphi^{0}\left(\bmod \underline{G}_{\mathrm{ak}}(\operatorname{omb}(\mathfrak{X}))\right),
$$

d'où

$$
g \equiv(\bar{f} f) \circ^{u} \varphi^{0}\left(\bmod \underline{G}_{\mathrm{ak}}(\operatorname{omb}(\mathfrak{X}))\right) .
$$

On achève ainsi la preuve que $\rho$ est une bijection dans le cas d'un corps de base $k$.

Remarque 2.35. (a) Soit $k$ un corps parfait. La preuve du fait que $\rho$ est une bijection pour un k-groupe unipotent connexe $U$ peut se faire à partir de: "Tout k-groupe unipotent connexe $U$ est isomorphe à un sous groupe fermé d'un produit fini $\prod\left(W_{r(i)}(k)^{+}\right)^{(i)}$ ". On peut ensuite montrer que $\rho$ est un isomorphisme pour $W_{r}(k)^{+}$à l'aide du symbole de Witt, qui constitue l'extension naturelle de l'opération $\operatorname{Res}(F(T) d u / u)$ définie plus haut ce qui donne que $\rho$ est un isomorphisme pour $\prod\left(W_{r(i)}(k)^{+}\right)^{(i)}$, et à fortiori pour tout sous-groupe fermé.

(b) A quelques détails près la preuve que l'on vient de donner permet de montrer que $\rho$ est une bijection pour tout $k$-schéma en groupes $G$ localement de présentation finie.

\section{Reduction de la preuve de (1.4.4) au cas où $A$ est noethérien et réduit}

Le but de ce numéro est la démonstration de la proposition suivante, qui ramène la preuve de (1.4.4) au cas d'une base réduite. On reprend les notations de (1.4.4).

Proposition 3.1. Soit A un anneau noethérien. On suppose que :

(i) Pour tout idéal q de A égal à son radical les flèches $\rho$ et $\rho^{+}$de (1.4.4) obtenues à partir de $A / q$ sont des bijections.

(ii) Il existe un idéal I de A de carré nul, c.à.d. $I^{2}=0$, tel que les flèches $\rho$ et $\rho^{+}$de (1.4.4) obtenues à partir de A/I sont des bijections. Alors les flèches $\rho$ et $\rho^{+}$de (1.4.4) obtenues à partir de A sont des bijections.

Montrons d'abord comment (3.1) à partir de la Proposition (3.1) on ramène la preuve de (1.4.4) au cas où $A$ est noethérien et réduit. Soit $N \subset A$ 
le nilradical de $A$. Il existe alors un entier $m \geq 0$ tel que $N^{m+1}=0$, et une chaîne finie

$$
0=N^{m+1} \subset N^{m} \subset \ldots \subset N \subset A .
$$

Posons $A^{(j)}=A / N^{j+1}$ pour $0 \leq j \leq m$. L'idéal $N^{j} / N^{j+1}$ de $A^{(j)}$ est de carré nul. Si l'on suppose (1.4.4) pour tout anneau réduit et noethérien on a la condition (i) de (3.1) assurée pour $A^{(j)}$. (Pour tout idéal $q \subset A^{(j)}$ égal à son radical on a a que $A^{(j)} / q$ est réduit). On démontre alors par récurrence sur l'entier $m$ que (3.1) entraîne (1.4.4) pour $A$ noethérien.

Soit $I \subset A$ un idéal de carré nul. Posons $S=\operatorname{Spec}(A), A_{0}=A / I$, et $S_{0}=\operatorname{Spec}\left(A_{0}\right)$. Étant donné un $S$-schéma $X$ on note $X_{0}$ le produit $X \times_{S} S_{0}$. On désigne par $S_{\text {fppf }}\left(\operatorname{resp} .\left(S_{0}\right)_{\text {fppf }}\right.$ ) le grand site fppf au-dessus de $S$ (resp. $\left.S_{0}\right)$. Soit $i:\left(S_{0}\right)_{\text {fppf }} \longrightarrow S_{\text {fppf }}$ le morphisme naturel. Soit $G$ un $S$-schéma en groupes lisse, commutatif, et séparé. Soit $\omega_{G / S}^{1}$ la restriction du $\mathcal{O}_{S}$-module des différentielles $\Omega_{G / S}^{1}$ à la section unité de $G$, c.à.d.

$$
\omega_{G / S}^{1}=e^{*}\left(\Omega_{G / S}^{1}\right),
$$

où $e: S \longrightarrow G$ désigne la section unité.

Soit $Y$ un $S$-schéma. Soit $G^{+}$le $S$-faisceau (fppf) en groupes défini par

$$
\operatorname{Hom}_{S}\left(Y, G^{+}\right)=\operatorname{Hom}_{S_{0}}\left(Y_{0}, G_{0}\right) .
$$

On a alors $G^{+}=i_{*} i^{*} G$. On définit le $S$-faisceau en groupes $K$ à partir de la suite exacte de faisceaux :

$$
1 \longrightarrow K \longrightarrow G \longrightarrow G^{+} \longrightarrow 1 .
$$

Noter que $G \longrightarrow G^{+}$est un epimorphisme car $G$ est $S$-lisse. On a la description suivante du faisceau $K$. Soit $S^{\prime}$ un $S$-schéma affine

$$
K\left(S^{\prime}\right)=\operatorname{Hom}_{\mathcal{O}_{S^{\prime}}}\left(\omega_{G / S^{\prime}}^{1}, \mathfrak{\Im} \mathcal{O}_{S^{\prime}}\right),
$$

où l'on désigne par $\mathcal{I}$ l'idéal de $\mathcal{O}_{S}$ donné par $I$.

La preuve de la proposition (3.1) mène à la considération d'une catégorie de $S$-faisceaux en groupes plus généraux que celle des $S$-schémas en groupes lisses.

DÉfinition 3.5. Soit $G$ un $S$-faisceau en groupes commutatif. On dit que G vérifie la propriété (A) (resp. (B)) si la flèche

$$
G(\operatorname{omb}(\mathfrak{X})) \times \operatorname{Hom}_{S-g r}\left(\mathfrak{J}^{0}, G\right) \longrightarrow G(\mathfrak{H})
$$


déduite à partir de $\varphi_{T}^{0}$ par composition et de l'opération de G est une bijection

$$
\left(\operatorname{resp} . \operatorname{Ext}_{S-g r}^{1}\left(\mathfrak{J}^{0}, G\right)=0 \text { et } H^{1}(\operatorname{omb}(\mathfrak{X}), G)=0\right)
$$

DÉFINITION 3.6. Un S-faisceau en groupes commutatifs est négligeable si $G\left(S^{\prime}\right)=0$ pour tout $S$-schéma plat $S^{\prime}$.

Proposition 3.7. Soit $G \longrightarrow G^{\prime}$ un homomorphisme de faisceaux en groupes commutatifs à noyau et conoyau négligeables. Alors pour tout $S$ schéma plat $S^{\prime}$ on $a$ :

(i) Le morphisme $H^{i}\left(S^{\prime}, G\right) \longrightarrow H^{i}\left(S^{\prime}, G^{\prime}\right)$, pour tout $i=0,1$, est un isomorphisme;

(ii) Pour tout S-schéma en groupes plat $\Gamma$ la flèche

$$
\operatorname{Ext}^{i}(\Gamma, G) \longrightarrow \operatorname{Ext}^{i}\left(\Gamma, G^{\prime}\right), \text { pour } i=0,1,
$$

est un isomorphisme.

PREUVE. $\quad$ de (i). Il résulte de [7 bis], Exp. IV, 4.10 que le foncteur qui a un faisceau en groupes abélien $G$ de $S_{\text {fppf }}$ associe sa restriction au petit site fppf de S est exact. D'où que G et sa restriction au petit site fppf ont la même cohomologie. En particulier, si $G$ est négligeable on a pour tout $i>0$, $H^{i}(S, G)=0$. L'affirmation résulte alors de la suite exacte de cohomologie.

On déduit formellement (ii) de (i) à partir de la description d'une extension de $\Gamma$ par $G$ en termes d'un $G$-torseur sur $\Gamma$ comme celle donnée dans [5].

C.Q.F.D.

On a les propriétés de permanence suivantes de la condition (A) (resp. (B)).

Corollaire 3.8. Soit $G \longrightarrow G^{\prime}$ comme dans (3.7). Alors G vérifie (A) (resp. (B)) si et seulement si $G^{\prime}$ vérifie (A) (resp. (B)). (Noter que $\mathfrak{J}^{0}$, omb( $\mathfrak{X})$ et $\mathfrak{U}$ sont des $S$-schémas plats).

Proposition 3.9. Soit $0 \longrightarrow G^{\prime} \longrightarrow G \longrightarrow G^{\prime \prime} \longrightarrow 0$ une suite exacte de S-faisceaux en groupes abéliens. On a alors :

(i) Si $G^{\prime}$ et $G^{\prime \prime}$ vérifient (B) alors $G$ vérifie $(B)$.

(ii) Si $G^{\prime}$ vérifie $(A)$ et $(B)$ et $G^{\prime \prime}$ vérifie $(A)$ alors $G$ vérifie $(A)$.

(iii) Si $G^{\prime}$ et $G^{\prime \prime}$ vérifient $(A)$ et $(B)$ alors $G$ vérifie $(A)$ et $(B)$. 
Preuve. de (i). Résulte trivialement de la suite exacte de cohomologie et de la suite exacte des Ext 's. L'affirmation (iii) résulte immédiatement de (i) et de (ii). Prouvons alors (ii). On a le diagramme commutatif de suite exactes :

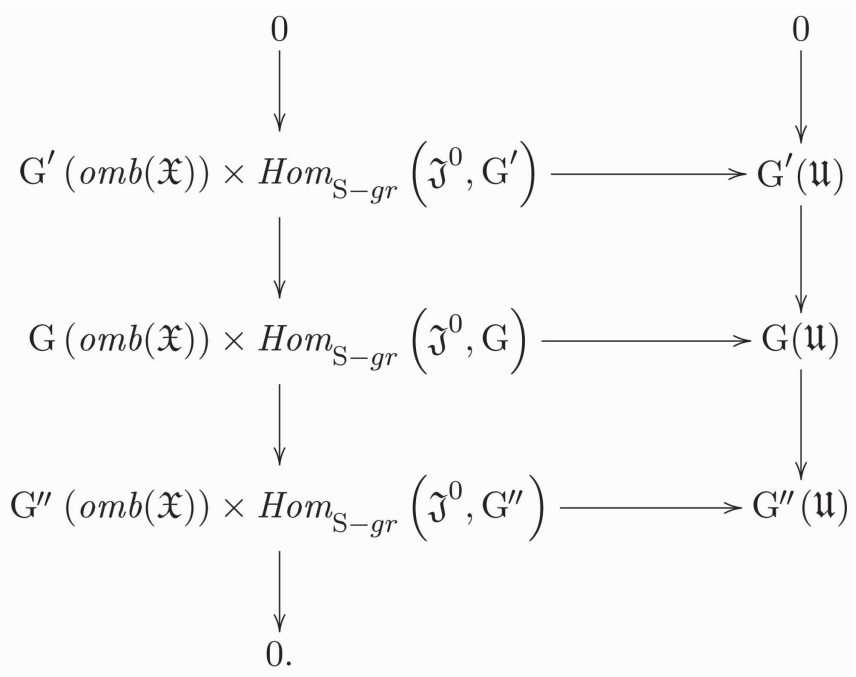

L'exactitude de la suite à gauche résulte de (B) pour $G^{\prime}$. La première et la troisième flèche horizontales sont des isomorphismes car $G^{\prime}$ et $G^{\prime \prime}$ vérifient (A).

$D^{\prime}$ autre part $G(\mathfrak{U}) \longrightarrow G^{\prime \prime}(\mathfrak{U})$ est un épimorphisme car $G^{\prime}$ vérifie (B). D'où la deuxième flèche horizontale est aussi un isomorphisme.

C.Q.F.D.

Soit $N$ un $\mathcal{O}_{S^{-}}$-module. Soit $W(N)$ le $S$-groupe vectoriel défini par

$$
W(N)\left(S^{\prime}\right)=\Gamma\left(S^{\prime}, \mathcal{O}_{S^{\prime}} \otimes_{\mathcal{O}_{S}} N\right)
$$

pour tout $S$-schéma $S^{\prime}$.

Il est clair que $W(N)$ est un faisceau fppf.

Proposition 3.11. (i) Tout faisceau négligeable $G^{\prime}$ vérifie $(A)$ et (B).

(ii) On suppose le théorème (1.4.4) démontré pour $S$ affine et noethérien. Alors $\underline{G}_{\mathrm{aS}}$ vérifie $(A)$ et $(B)$.

(iii) Soit $S^{\prime}$ un sous-schéma fermé de $S$ et soit $S^{\prime} \hookrightarrow S$ l'immersion canonique. On suppose le théorème (1.4.4) démontré pour $S^{\prime}$. Alors $W\left(\mathcal{O}_{S^{\prime}}\right)=i_{*} \underline{G}_{\mathrm{aS}^{\prime}}$ vérifie $(A)$ et $(B)$. 
Preuve. L'affirmation (i) résulte immédiatement de (3.8). Voyons comment (iii) se démontre à partir de (ii). Notons d'abord que

$$
\begin{gathered}
\Gamma\left(\operatorname{omb}(\mathfrak{X}), i_{*} \underline{G}_{a S^{\prime}}\right)=\Gamma\left(\operatorname{omb}(\mathfrak{X})_{S^{\prime}}, \underline{G}_{a S^{\prime}}\right) \\
\left(\operatorname{resp} . H^{1}\left(\operatorname{omb}(\mathfrak{X}), i_{*} \underline{G}_{a S^{\prime}}\right)=H^{1}\left(\operatorname{omb}(\mathfrak{X})_{S^{\prime}}, \underline{G}_{a S^{\prime}}\right)\right),
\end{gathered}
$$

et

$$
\operatorname{Hom}_{S-g r}\left(\mathfrak{J}^{0}, i_{*} \underline{G}_{a S^{\prime}}\right)=\operatorname{Hom}_{S^{\prime}-g r}\left(\mathfrak{J}_{S^{\prime}}^{0}, \underline{G}_{a S^{\prime}}\right)
$$

Il en résulte trivialement que (A) pour $\underline{G}_{a S^{\prime}}$ donne (A) pour $i_{*} G_{a S^{\prime}}$.

Comme $\mathfrak{J}^{0}$ est affine et $i_{*} \underline{G}_{a S^{\prime}}=W\left(\mathcal{O}_{S^{\prime}}\right)$ alors Ext $\operatorname{Ex}_{S-g r}\left(\mathfrak{J}^{0}, i_{*} \underline{G}_{a S^{\prime}}\right)$ est isomorphe $\grave{a}$

$$
H^{2}\left(\mathfrak{J}^{0}, i_{*} \underline{G}_{a S^{\prime}}\right) \simeq H^{2}\left(\mathfrak{J}_{S^{\prime}}^{0}, \underline{G}_{a S^{\prime}}\right) .
$$

Or ce dernier groupe est isomorphe à

$$
\operatorname{Ext}_{S^{\prime}-g r}\left(\mathcal{J}_{S^{\prime}}^{0}, i_{*} \underline{G}_{a S^{\prime}}\right)=0
$$

d'où

$$
\operatorname{Ext}_{S-g r}\left(\mathfrak{I}^{0}, i_{*} \underline{G}_{a S^{\prime}}\right)=0 .
$$

Donc (B) pour $\underline{G}_{a S^{\prime}}$ donne (B) pour $i_{*} \underline{G}_{a S^{\prime}}$. Prouvons maintenant (ii). On a vu que les extensions de $\mathfrak{J}^{0}$ par $\underline{G}_{a S}$ sont classées par le groupe $H^{2}\left(\mathfrak{J}^{0}, \underline{G}_{a S}\right)$. Comme $\underline{G}_{a S}$ est un $S$-schéma de présentation finie et $\mathfrak{J}^{0}=\lim _{\overleftarrow{n}} \mathfrak{J}_{n}^{0}$ on a

$$
H^{2}\left(\mathfrak{J}^{0}, \underline{G}_{a S}\right)=\lim _{\rightarrow} H^{2}\left(\mathfrak{I}_{n}^{0}, \underline{G}_{a S}\right) .
$$

Étant donnée une extension

$$
0 \longrightarrow \underline{G}_{a S} \longrightarrow E \longrightarrow \mathfrak{J}^{0} \longrightarrow 1
$$

il existe un entier $n \geq 0$ et une extension

$$
0 \longrightarrow \underline{G}_{a S} \longrightarrow E_{n} \stackrel{\pi}{\longrightarrow} \mathfrak{J}_{n}^{0} \longrightarrow 1
$$

tel que $E \simeq E_{n} \times_{\mathfrak{J}_{n}^{0}} \mathfrak{I}^{0}$. Notons par $\left(\varphi_{T}^{0}\right)_{n}$ l'image de $\varphi_{T}^{0} \operatorname{par} \mathfrak{I}^{0}(\mathfrak{H}) \longrightarrow \mathfrak{J}_{n}^{0}(\mathfrak{H})$. D'autre part

$$
E_{n}(\mathfrak{U}) \longrightarrow \mathfrak{J}_{n}^{0}(\mathfrak{U})
$$

est une surjection car $H^{1}\left(\mathfrak{H}, \underline{G}_{a S}\right)=0, \mathfrak{U}$ étant un schéma affine. Il existe un relèvement $\gamma \in E_{n}(\mathfrak{H})$ de $\left(\varphi_{T}^{0}\right)_{n}$ qui rend commutatif le diagramme suivant 


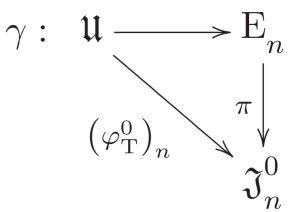

Le S-groupe $E_{n}$ est représentable par un $S$-schéma lisse et séparé de présentation finie en tant qu'extension de $\mathfrak{J}_{n}^{0}$ par $\underline{G}_{a S}$. L'hypothèse entraîne qu'il existe un entier $m \geq n$ et un $S$-homomorphisme

$$
h: \mathfrak{I}_{m}^{0} \longrightarrow E_{n}
$$

tel que

$$
\gamma \equiv h \circ\left(\varphi_{T}^{0}\right)_{m}\left(\bmod E_{n}(\operatorname{omb}(\mathfrak{X}))\right),
$$

d'où

$$
\pi \circ h: \mathfrak{I}_{m}^{0} \longrightarrow \mathfrak{J}_{n}^{0}
$$

vérifie :

$$
\left(\varphi_{T}^{0}\right)_{n} \equiv(\pi \circ h) \circ\left(\varphi_{T}^{0}\right)_{m}\left(\bmod \mathfrak{J}_{n}^{0}(\operatorname{omb}(\mathfrak{X}))\right) .
$$

La propriété d'unicité de la factorisation donne que $\pi$ ○ h coüncide avec le morphisme canonique de $\mathfrak{I}_{m}^{0}$ dans $\mathfrak{I}_{n}^{0}$. On a ainsi le diagramme commutatif:

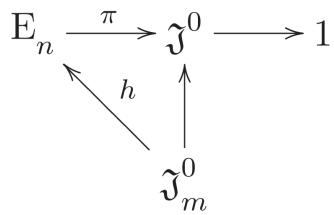

L'extension $E_{m}=E_{n} \times_{\mathfrak{J}_{n}^{0}} \mathfrak{I}_{m}^{0}$ de $\mathfrak{I}_{m}^{0}$ par $\underline{G}_{a S}$ est scindée. En effet on vérifie immédiatement que le couple $\left(h, I d_{\mathfrak{J}_{m}^{0}}\right)$ est un scindage de $E_{m}$. D'autre part $E \simeq E_{m} \times_{\mathfrak{J}_{m}^{0}} \mathfrak{J}^{0}$ ce qui prouve bien (ii).

C.Q.F.D.

Proposition 3.12. On suppose vérifié l'hypothèse (i) de (3.1).

Soit $N$ un $\mathcal{O}_{S}$-module de type fini. Alors $W(N)$ vérifie $(A)$ et $(B)$.

Preuve. Comme $\mathrm{N}$ est un $\mathcal{O}_{S}$-module de type fini et $\mathrm{S}$ est noethérien on peut trouver une suite de composition.

$$
0=N_{0} \subset N_{1} \subset \ldots \subset N_{p}=N
$$


tel que $N_{i+1} / N_{i}(0 \leq i \leq p-1)$ soit isomorphe au $\mathcal{O}_{S}$-module donné par un $A$-module de la forme $A / q$, où $q$ désigne un idéal premier de $A$. La démonstration se fait par récurrence sur la longueur de la suite (*). Pour $p=1$ l'énoncé résulte de (3.11), (iii). Si $p>1$ on a une suite exacte

$$
0 \longrightarrow F \longrightarrow W\left(N_{p-1}\right) \longrightarrow W(N) \longrightarrow W(A / q) \longrightarrow 0
$$

où $F$ est négligeable. Alors $\operatorname{Im}\left(W\left(N_{p-1}\right) \longrightarrow W(N)\right)$ vérifie $(A)$ et $(B)$ d'après (3.8). On conclut par (3.9), (iii).

C.Q.F.D.

Proposition 3.13. Même hypothèse que dans (3.12). Le S-faisceau en groupes $K$ défini dans (3.3) vérifie $(A)$ et $(B)$.

Preuve. Posons $N=\underline{H o m}_{\mathcal{O}_{S}}\left(\omega_{G / S}^{1}, \mathfrak{I}\right)$. On a alors un morphisme naturel de $S$-faisceaux $W(N) \longrightarrow K$ donné pour tout $S$-schéma $S^{\prime}$ par la flèche naturelle

$\Gamma\left(S^{\prime}, \underline{\operatorname{Hom}}_{\mathcal{O}_{S}}\left(\omega_{G / S}^{1}, \mathfrak{I}\right) \otimes_{\mathcal{O}_{S}} \mathcal{O}_{S^{\prime}}\right) \longrightarrow \Gamma\left(S^{\prime}, \underline{H o m}_{\mathcal{O}_{S^{\prime}}}\left(\omega_{G / S^{\prime}}^{1}, \mathfrak{I} \mathcal{O}_{S^{\prime}}\right)\right)(c f$.

Comme $\omega_{G / S}^{1}$ est localement libre ce morphisme est surjectif à noyau négligeable. L'affirmation résulte alors de (3.8) et de (3.12).

C.Q.F.D.

Preuve. de (3.1). D'après (ii) $G_{0}=G \times_{S} S_{0}$ vérifie $(A)$, alors $G^{+}$vérifie (A) comme on le voit immédiatement par définition de $G^{+}$. D’après (3.13) $K$ vérifie (A) et (B). Donc G satisfait (A) d'après (3.9), (ii).

C.Q.F.D.

On reprend les notations de (1.4.4).

Proposition 3.14. Soit $A$ un anneau artinien. Les flèches

$$
\operatorname{Hom}_{S-g r}\left(\mathfrak{J}^{0}, G\right) \stackrel{\rho}{\longrightarrow} F(G)
$$

et

$$
\operatorname{Hom}_{S-g r}(\mathfrak{J}, G) \stackrel{\rho^{+}}{\longrightarrow} F^{+}(G)
$$

sont des isomorphismes.

REMARQue 3.15. Dans (3.14) le fait que G soit séparé n'intervient pas.

Le dévissage donné par la Proposition (3.1) ramène la preuve de (3.14) au cas d'un corps de base, et l'on sait que tout k-schéma en groupes est séparé. 
Notation 3.16. Soit A un anneau local complet et $m_{A}$ son idéal maximal. Si X (resp. $f: X \longrightarrow Y$ ) est un A-schéma (A-morphisme) on désigne par $\widehat{X}$ (resp. $\widehat{f})$ le complété formel de $X$ le long de $X^{\prime}=X \times_{A}\left(\operatorname{Spec}\left(A / m_{A}\right)\right)$ (resp. le prolongement def aux complétés). Noter que si G est un A-schéma en groupes alors $\widehat{G}$ est muni d'une structure de groupe.

Le résultat suivant nous sera utilise par la suite

Proposition 3.17. Soit A un anneau local complet. Soit G un A-schéma en groupes lisse. Soit $\alpha \in G\left(A[[t]]\left[t^{-1}\right]\right)=G(\mathfrak{H})$. Il existe un $A$-homomorphisme unique

$$
f: \widehat{\mathfrak{J}}^{0} \longrightarrow \widehat{G}
$$

et une section $\delta$ de $G$ au-dessus de $\mathfrak{X}=\operatorname{Spf}(A[[t]])$ tel que

$$
\widehat{\alpha}=\left(f \circ \widehat{\varphi}_{T}\right) \delta \text {. }
$$

Preuve. Posons

$$
\begin{gathered}
\mathfrak{l}^{(n)}=\mathfrak{U} \times_{A} \operatorname{Spec}\left(A / m_{A}^{n+1}\right), \\
\alpha^{(n)}=\alpha \times_{A} \operatorname{Spec}\left(A / m_{A}^{n+1}\right), \\
G^{(n)}=G \times_{A} \operatorname{Spec}\left(A / m_{A}^{n+1}\right), \ldots \text { etc. }
\end{gathered}
$$

D’après (3.14) on a pour tout entier $n \geq 0$ un unique $A / m_{A}^{n+1}$-homomorphisme

$$
f^{(n)}:\left(\mathfrak{J}^{0}\right)^{(n)} \longrightarrow G^{(n)}
$$

tel que

$$
\delta^{(n)}=\alpha^{(n)}\left(f^{(n)} \circ\left(\varphi_{T}^{0}\right)^{(n)}\right)^{-1} \in G\left(\mathfrak{X}^{(n)}\right) .
$$

On construit ainsi, de proche en proche, un système projectif $f=\left(f^{(n)}\right)(n \geq 0)$ de A-homomorphismes et un autre $\delta=\left(\delta^{(n)}\right)(n \geq 0)$ de $A$-morphismes dans $G$. Il est clair que $f$ et $\delta$ satisfont à la condition de l'énoncé. (Noter que comme A[[T]] est local complet, $\delta$ provient d'un élément de $G(\operatorname{omb}(\mathfrak{X}))$.)

C.Q.F.D.

\section{Preuve de (1.4.4) dans le cas d'un trait complet $S$ de base}

(4.1). On désigne par $V$ un anneau de valuation discrète complet, par $m$ son idéal maximal, et par $K$ son corps des fractions. On pose 
$S=\operatorname{Spec}(V)$ (trait complet). Soit $k=V / m$ le corps résiduel de $V$ que l'on peut supposer algébriquement clos par (1.4.6) et par (6.82) de [8].

Soit $G$ un $S$-schéma en groupes lisse commutatif et séparé. Soit $G^{0} \subset G$ le $S$-sous-groupe ouvert dont les fibres géométriques sont les composantes connexes de l'indentité (composantes neutre de $G^{0}$ ).

On reprend ici les notations de (1.3). On pose $\mathfrak{X}=\mathfrak{X}_{(0)}$ (complété formel de $\mathbb{P}_{S}^{1}$ le long de $\left.D_{(0)}\right)$ et $B=\Gamma\left(\mathfrak{X}, \mathcal{O}_{\mathfrak{X}}\right)$. On note par $T$ (resp. $T^{\prime}$ ) la section de $B$ (resp. $\left.B_{(\infty)}\right)$ donnée par $\mathfrak{T}$ (resp. $\mathfrak{T}^{-1}$ ), où $\mathfrak{T}$ désigne la fonction méromorphe de $\mathbb{P}_{S}^{1}$ définie dans (1.3). On a alors $B=V[[T]]$ (resp. $B_{(\infty)}=$ $\left.V\left[\left[T^{\prime}\right]\right]\right)$,

$$
\begin{gathered}
\operatorname{omb}\left(\mathfrak{X}_{(0)}\right)=\operatorname{omb}(\mathfrak{X})=\operatorname{Spec}(V[[T]]) \quad\left(\operatorname{resp} . \operatorname{omb}\left(\mathfrak{X}_{(\infty)}\right)=\operatorname{Spec}\left(V\left[\left[T^{\prime}\right]\right]\right)\right), \\
\left.\mathfrak{U}_{(0)}=\mathfrak{U}=\operatorname{Spec}\left(V[[T]]\left[T^{-1}\right]\right) \quad\left(\operatorname{resp} . \mathfrak{U}_{(\infty)}\right)=\operatorname{Spec}\left(V\left[\left[T^{\prime}\right]\right]\left[T^{\prime-1}\right]\right)\right),
\end{gathered}
$$

On pose $\mathfrak{J}={ }^{(0)} \mathfrak{J}$. Avec la notation (1.3.25) on a une section $\varphi_{\mathfrak{E}}^{1}\left(\right.$ resp. $\left.\varphi_{\mathfrak{C}}^{0}\right) \mathrm{de}$ $\mathfrak{I}\left(\right.$ resp. $\mathfrak{J}^{0}$ ) au-dessus de $U$ tel que la restriction à $\mathfrak{U}$ coïncide avec $\varphi_{T}^{1}$ (resp. $\left.\varphi_{T}^{0}\right)($ cf. (1.3.9)). On a la décomposition suivante

$$
\varphi_{\mathfrak{C}}^{0}=\mathfrak{C}^{-1}\left(1-\mathfrak{T}^{-1} T\right)^{-1} \quad\left(\operatorname{resp} . \varphi_{T}^{0}=t^{-1}\left(1-t^{-1} T\right)^{-1}\right)
$$

qui correspond à la décomposition en produit direct $\mathfrak{J}^{0}=\underline{G}_{m S} \times \wedge^{0}$. On note par ${ }^{m} \varphi_{\mathfrak{C}}^{0}\left(\right.$ resp. ${ }^{m} \varphi_{T}^{0}$ ) la section de $\mathbb{G}_{m S}$ au-dessus de $U$ (resp. $\left.\mathfrak{U}\right)$ donnée par $\mathbb{T}^{-1}$ (resp. $\left.t^{-1}\right)$, et par ${ }^{u} \varphi_{\mathfrak{C}}^{0}$ (resp. ${ }^{u} \varphi_{T}^{0}$ ) la section de $\wedge^{0}$ au-dessus de $U$ (resp. $\mathfrak{U})$ donnée par $1-\mathfrak{C}^{-1} T\left(\right.$ resp. $\left.1-t^{-1} T\right)$.

Lemme 4.1.2. Soit $\alpha \in G(\mathfrak{H})$. Il existe alors $\alpha^{\prime} \in G^{0}(\mathfrak{H})$ tel que

$$
\alpha \equiv \alpha^{\prime}(\bmod G(\operatorname{omb}(\mathfrak{X}))) .
$$

Preuve. Soit $\alpha_{k}$ l'image de $\alpha$ dans $G\left(\mathfrak{U}_{\{k\}}\right)(k=V / m)$. Par [6] on a une suite exacte de k-groupes

$$
1 \longrightarrow G_{k}^{0} \longrightarrow G_{k} \stackrel{\rho}{\longrightarrow} \Gamma_{k} \longrightarrow 1,
$$

où $\Gamma_{k}$ désigne un k-groupe étale constant. La section $p\left(\alpha_{k}\right)$ se prolonge en une section $\overline{p\left(\alpha_{k}\right)}$ de $\Gamma_{k}$ au-dessus de Spec $(k[[T]])=\operatorname{omb}\left(\mathfrak{X}_{k}\right)$. Il existe alors un relèvement $\beta_{k}$ de $\overline{p\left(\alpha_{k}\right)} \grave{a} G_{k}, G_{k}^{0}$ étant lisse. Comme $G$ est un $S$-schéma lisse, la section $\beta_{k}$ de $G$ au-dessus de Spec $((V / m)[[T]])$ se relève en une section $\beta$ de $G$ au-dessus de $\mathfrak{X}$ et à fortiori au-dessus de $\operatorname{omb}(\mathfrak{X})=\operatorname{Spec}(V[[T]])$. Puisque $\alpha_{k} \beta_{k}^{-1} \in G^{0}\left(\mathfrak{H}_{k}\right)$ (par construction de $\beta$ ) et comme $G^{0}$ est ouvert il existe un voisinage ouvert $\mathcal{V}$ de $\mathfrak{U}_{k}=\operatorname{Spec}\left(k[[T]]\left[T^{-1}\right]\right)$ dans $\mathfrak{U}$ tel que $\alpha \cdot \beta^{-1}(\mathcal{V}) \subset G^{0}$. Alors $\alpha^{\prime}=\alpha \cdot \beta^{-1}$ vérifie la condition de l'énoncé. En effet l'ouvert $\alpha^{-1}\left(G^{0}\right)$ contient la fibre 
fermée $\mathfrak{H}_{k}$, et induit sur la fibre générique $\mathfrak{H}_{K}$ un ouvert fermé (car $G_{K}^{0}$ est ouvert et fermé dans $G_{K}$ ) non-vide contenant $U_{K}$ donc égal à $\mathfrak{H}_{K}$ $\left(\mathfrak{U}=\mathfrak{U}_{k} \amalg \mathfrak{H}_{K}\right)$.

C.Q.F.D.

Remarque 4.1.3. On peut, d'après (4.1.2), supposer par la suite G à fibre connexes, c.à.d. $G=G^{0}$, et donc qu'il est représentable par un $S$ schéma de présentation finie.

Notons que si le théorème (1.4.4) est vrai alors la décomposition en produit direct $\mathfrak{J}^{0}=\underline{\mathrm{G}}_{m S} \times \wedge^{0}$ doit nécessairement se refléter dans $G(\mathfrak{H}) / G(o m b(\mathfrak{X}))$.

Soit $\alpha=h \circ \varphi_{T}^{0}$, la décomposition

$$
\varphi_{T}^{0}=\left({ }^{m} \varphi_{T}^{0},{ }^{u} \varphi_{T}^{0}\right) \in \mathrm{G}_{m S}(\mathfrak{U}) \times \wedge^{0}(\mathfrak{U})
$$

donne lieu à deux sections ${ }^{m} \alpha=h \circ^{m} \varphi_{T}$ et $^{u} \alpha=h \circ^{u} \varphi_{T}$ de Gau-dessus de

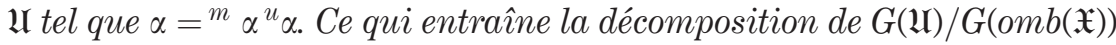
en produit direct.

On reprend ici les notations de (3.17) avec $A=V$ et $m_{A}=m$. Soit $\alpha \in G(\mathfrak{l})$, d'après (3.17) quitte à multiplier $\alpha$ par $\delta \in G(\operatorname{omb}(\mathfrak{X}))$, on peut supposer que pour tout entier $n \geq 0$ il existe un $V / m^{n+1}$-homomorphisme

$$
f^{(n)}:\left(\mathfrak{J}^{0}\right)^{(n)} \longrightarrow G^{(n)}
$$

avec $\alpha^{(n)}=f^{(n)} \circ\left(\varphi_{T}^{0}\right)^{(n)}$. On pose $\left(\varphi_{T}^{0}\right)^{(n)}=\left(\left({ }^{m} \varphi_{T}^{0}\right)^{(n)},\left({ }^{n} \varphi_{T}^{0}\right)^{(n)}\right)$ et

$$
\begin{gathered}
{ }^{m} \alpha^{(n)}=f^{(n)} \circ\left({ }^{m} \varphi_{T}^{0}\right)^{(n)} \\
\left(\text { resp. }{ }^{u} \alpha^{(n)}=f^{(n)} \circ\left({ }^{u} \varphi_{T}^{0}\right)^{(n)}\right) .
\end{gathered}
$$

On pose $f=\left(f^{(n)}\right) \in \operatorname{Hom}_{S-g r}\left(\widehat{\mathfrak{J}}^{0}, \widehat{G}\right)$. On a alors avec la convention (3.16) :

$$
\widehat{\alpha}=f \circ \widehat{\varphi}_{T}^{0} \text {. }
$$

On pose ${ }^{m} \varphi^{0}={ }^{m} \varphi_{T}^{0},{ }^{u} \varphi^{0}={ }^{u} \varphi_{T}^{0}$ et on introduit maintenant les $S$ morphismes formels suivants

$$
{ }^{m} \widehat{\alpha}=f \circ{ }^{m} \widehat{\varphi}^{0}: \widehat{\mathfrak{U}} \longrightarrow \widehat{G}
$$

et

$$
{ }^{u} \widehat{\alpha}=f \circ{ }^{u} \widehat{\varphi}^{0}: \widehat{\mathfrak{U}} \longrightarrow \widehat{G} .
$$

En fait on a ${ }^{m} \widehat{\alpha}=\left({ }^{m} \alpha^{(n)}\right)$ (resp. $\left.{ }^{u} \widehat{\alpha}=\left({ }^{u} \alpha^{(n)}\right)\right)(n \geq 0)$, et $\widehat{\alpha}={ }^{m} \widehat{\alpha}^{u} \widehat{\alpha}$. 


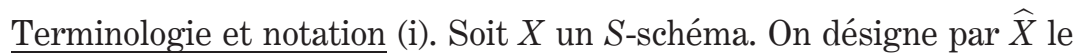
complété formel de $X$ pour la topologie $m$-adique et on l'appelle la fibre formelle de $X$. Soit $f: X \longrightarrow Y$ un morphisme de $S$-schémas. On désigne par $\widehat{f}: \widehat{X} \longrightarrow \widehat{Y}$ le $S$-morphisme de schémas formels induit par $f$ et on appelle la fibre formelle de $f$ (cf. (3.16)).

(ii) Soit $n \geq 1$. On désigne toujours par $\varphi_{T}^{0}=\varphi^{0}$ le morphisme composé

$$
\mathfrak{U} \stackrel{\varphi_{T}^{0}}{\longrightarrow} \mathfrak{J}^{0} \longrightarrow \mathfrak{I}_{n}^{0}
$$

Expliquons la stratégie de la preuve de (1.4.4) pour un trait complet de base $S$. On introduit à partir du morphisme formel

$$
\widehat{\alpha}\left(\text { resp. }{ }^{m} \widehat{\alpha},{ }^{u} \widehat{\alpha}\right): \widehat{\mathfrak{U}} \longrightarrow G
$$

(cf. (4.1.5) et (4.1.5) bis) un $S$-morphisme formel

$$
\bar{\alpha}\left(\text { resp. }{ }^{m} \bar{\alpha},{ }^{u} \bar{\alpha}\right): \widehat{U} \longrightarrow G
$$

redonnant $\widehat{\alpha}$ (resp. $\left.{ }^{m} \widehat{\alpha},{ }^{u} \widehat{\alpha}\right)$ par composition avec $\widehat{\mathfrak{U}}=\widehat{\mathfrak{U}}_{(0)} \longrightarrow \widehat{U}$ (cf. (4.1.19), (ii) et (4.1.24)). Dans (4.1.6) on prouve que ${ }^{m} \widehat{\alpha}$ et ${ }^{n} \widehat{\alpha}$ sont algébrisables. Pour cela il faut considérer la trace $\operatorname{tr}_{\theta_{n}} \alpha\left(\right.$ resp. $\left.\operatorname{tr}_{\Theta_{n}} \bar{\alpha}\right)$ de $\alpha(\operatorname{resp} . \bar{\alpha})$ par rapport à un certain morphisme fini $\theta_{n}: \mathfrak{U} \longrightarrow \mathfrak{U}$ (resp. $\Theta: U \longrightarrow U$ ) (cf. (4.1.8) et (4.1.9)), et utiliser une propriété de pureté pour les sections d'un $S$-schéma en groupes à valeur dans omb(x) (cf. (4.1.15)). On montre finalement que ${ }^{m} \widehat{\alpha}$ est égal à la fibre formelle de $\operatorname{tr}_{\theta_{n}} \alpha$ pour $n$ convenable. $\mathrm{Au}$ (4.2) on démontre le lemme d'algébrisation (4.2.1) pour un morphisme $\psi: \operatorname{omb}(\widehat{U})_{K} \longrightarrow \mathbb{P}_{K}^{N}$ dont la composition avec

$$
\operatorname{omb}\left(\widehat{\mathfrak{U}}_{(0)}\right)_{K}\left(\operatorname{resp} \operatorname{omb}\left(\widehat{\mathfrak{U}}_{(\infty)}\right)_{K}\right) \longrightarrow \operatorname{omb}(\widehat{U})_{K}
$$

provient d'un morphisme

$$
\mathfrak{U}_{(0) K}\left(\text { resp. } \mathfrak{U}_{(\infty) K}\right) \longrightarrow \mathbb{P}_{K}^{N}
$$

A l'aide du lemme cité on prouve que ${ }^{m} \bar{\alpha}$ et ${ }^{u} \bar{\alpha}$ sont algébrisables dans un sens précisé au lemme (4.3.8). A partir de ce fait on prouve que ${ }^{m} f$ et ${ }^{u} f$ sont algébrisables (cf. Propositions (4.3.13) et (4.4.24)).

On démontre d'abord la

Proposition 4.1.6. Le S-morphisme formel $m \widehat{\alpha}: \widehat{\mathfrak{l}} \longrightarrow \widehat{G}$ (resp. $u \widehat{\alpha}: \widehat{\mathfrak{U}} \longrightarrow \widehat{G})$ est algébrisable, c.à.d. il existe un $S$-morphisme

$$
{ }^{m} \alpha: \mathfrak{U} \longrightarrow G\left(\operatorname{resp} .{ }^{u} \alpha: \mathfrak{U} \longrightarrow G\right)
$$

qui redonne ${ }^{m} \widehat{\alpha}\left(\right.$ resp. $\left.{ }^{u} \widehat{\alpha}\right)$ par passage à la fibre formelle. On $a \alpha={ }^{m} \alpha^{u} \alpha$. 
Notons que l'on a :

$$
U=\mathbb{P}_{S}^{1}-D=\operatorname{Spec}\left(V\left[\mathbb{T}, \mathbb{T}^{-1}\right]\right)(\operatorname{cf} .(1.3 .4))
$$

Soit

$$
\Theta: U=\mathbb{P}_{S}^{1}-D \longrightarrow \mathbb{P}_{S}^{1}-D
$$

le $S$-morphisme fini correspondant au $V$-homomorphisme $V\left[\mathfrak{T}, \mathfrak{T}^{-1}\right] \longrightarrow$ $V\left[\mathfrak{T}, \mathfrak{T}^{-1}\right]$ faisant correspondre $\mathfrak{T}^{n}$ à $\mathfrak{T}$. Soit

$$
\theta_{n}: \mathfrak{U} \longrightarrow \mathfrak{U}
$$

la restriction de $\Theta_{n}$ à $\mathfrak{U}$, c.à.d. le $S$-morphisme fini qui correspond au $V$ homomorphisme $V[[T]]\left[T^{-1}\right] \longrightarrow V[[T]]\left[T^{-1}\right]$ défini par $T \longrightarrow T^{n}$. On confère à [7], Ch. XVII, 6.2.3 pour la preuve du résultat suivant

Proposition 4.1.10. On peut d'une et d'une seule façon définir, pour tout morphisme $f: X \longrightarrow S$, séparé, plat de présentation finie, et quasifini, et tout faisceau abélien $F$ sur $S$, un morphisme trace

$$
T r_{f}: f_{!} f^{*} F \longrightarrow F
$$

(On désigne par $f_{!}, F$ le sous-faisceau de $f_{*} F$ dont les sections sur un $U$ étale sur $S$ sont les sections de $F$ sur $U \times_{S} X$ dont le support est propre au-dessus de $U$ ) redonnant le morphisme trace habituel si $S$ est le spectre d'un corps, de sorte que les conditions suivantes soient vérifiées :

(i) $\mathrm{Tr}_{f}$ est fonctoriel sur $F$.

(ii) $T r_{f}$ est compatible à tout changement de base.

Lemme 4.1.11. On a les égalités suivantes :

$$
\operatorname{tr}_{\theta_{n}} \varphi_{T}^{0}=(-1)^{n+1}\left(t-T^{n}\right)^{-1}
$$

et

$$
\operatorname{tr}_{\theta_{n}} \varphi_{T}^{1}=(-1)^{n+1} T^{n}\left(t-T^{n}\right)^{-1} .
$$

PREUVE. En vertu de (ii) de (4.1.10) il suffira de montrer l'égalité en se plaçant sur Z, et comme I est séparé au-dessus de Z, il suffit de la vérifier après le changement de base

$$
\operatorname{Spec}(\mathrm{Q}) \longrightarrow \operatorname{Spec}(\mathrm{Z}) .
$$

Soit $\mathrm{Q}^{\prime}$ l'extension de $\mathrm{Q}$ engendrée par les racines $n$-ème de l'unité : $\theta_{1}, \theta_{2}, \ldots, \theta_{n}$. Il suffit alors de calculer la trace de la section 
$(t-T)^{-1} \in \mathfrak{J}\left(Q^{\prime}\left[t, t^{-1}\right]\right)$ par rapport au morphisme étale

$$
\operatorname{Spec}\left(\mathbb{Q}^{\prime}\left[t, t^{-1}\right]\right) \longrightarrow \operatorname{Spec}\left(\mathbb{Q}^{\prime}\left[t^{\prime}, t^{\prime-1}\right]\right),
$$

donné par $t^{\prime} \longrightarrow t^{n}$. Or on a l'égalité :

$$
\left(\theta_{1} t-T\right)^{-1}\left(\theta_{2} t-T\right)^{-1} \ldots\left(\theta_{n} t-T\right)^{-1}=(-1)^{n+1}\left(t^{\prime}-T^{n}\right)^{-1} .
$$

Ce qui montre bien la première formule (par (ii) de (4.1.10)). La deuxième en résulte.

C.Q.F.D.

Lemme 4.1.12. Soit $k$ un corps et $G_{k}$ un k-groupe lisse. Soit $\alpha \in G_{k}\left(k[[T]]\left[T^{-1}\right]\right)$. Il existe un entier $n>0$ tel que

$$
\operatorname{tr}_{\theta_{n^{\prime}}} \alpha \equiv t r_{\theta_{n^{\prime \prime}}} \alpha\left(\bmod G_{k}(k[[T]])\right)
$$

si $n^{\prime}, n^{\prime \prime} \geq n$, et si $n^{\prime}$ et $n^{\prime \prime}$ sont impairs.

Preuve. Puisque l'on a prouvé (1.4.4) pour le cas d'un corps de base (cf. § 2), on peut supposer qu'il existe un entier $n>0$ et un k-homomorphisme $h: \mathfrak{J}_{n}^{0} \longrightarrow G_{k}$ tel que $\alpha=h \circ \varphi_{T}^{0}$. Il suffit alors de vérifier l'affirmation pour a égal au morphisme composé $\mathfrak{U} \stackrel{\varphi_{T}^{0}}{\longrightarrow} \mathfrak{J}^{0} \longrightarrow \mathfrak{J}_{n}^{0}$ en vertu de $(i)$ de (4.1.10), ce qui immédiat à partir de (4.1.11). C.Q.F.D.

Si $F: X \longrightarrow Y$ est un $S$-morphisme rationnel du schéma $X$ dans un $S$-schéma séparé localement de type fini $Y$. On note par $\operatorname{dom}(F) \subset X$ le plus grand ouvert de définition de $F$ dans $X$. Soient

$$
\operatorname{pr}_{1}\left(\operatorname{resp} . \operatorname{pr}_{2}\right): \operatorname{omb}\left(\mathfrak{X} \times_{S} \mathfrak{X}\right) \longrightarrow \operatorname{omb}(\mathfrak{X})
$$

la projection sur le premier (resp. deuxième) facteur, et

$$
\Delta: \operatorname{omb}(\mathfrak{X}) \longrightarrow \operatorname{omb}\left(\mathfrak{X} \times_{S} \mathfrak{X}\right)
$$

le morphisme diagonal. Supposons que $G$ soit un $S$-schéma en groupes séparé et localement de type fini. Soit $\mathfrak{p}=(m, T) \subset V[[T]]$ l'idéal maximal engendré par $m$ et par $T$. Notons par $x \in \operatorname{omb}(\mathfrak{X})$ le point qui correspond à $\mathfrak{p}$. Soit

$$
F: \operatorname{omb}(\mathfrak{X})-\{x\} \longrightarrow G
$$

un $S$-morphisme. Soit

$$
H: \operatorname{omb}\left(\mathfrak{X} \times_{S} \mathfrak{X}\right) \longrightarrow G
$$


le $S$-morphisme rationnel défini par

$$
H=\left(F \circ \mathrm{pr}_{1}\right)\left(F \circ \mathrm{pr}_{2}\right)^{-1} .
$$

Proposition 4.1.15. Avec les notations ci-dessus on a que F admet un unique prolongement

$$
\bar{F}: \operatorname{omb}(\mathfrak{X}) \longrightarrow G \text {. }
$$

Prouvons d'abord le

Lemme 4.1.16. Soit $H$ défini comme ci-dessus. On a alors l'égalité suivante entre parties ouvertes de omb( $\mathfrak{X})$

$$
\operatorname{dom}(F)=\Delta^{-1}(\operatorname{dom}(H)) .
$$

Preuve. On a dom $(F) \subset \Delta^{-1}(\operatorname{dom}(H))$. En effet si $x \in \operatorname{dom}(F) \subset \operatorname{omb}(\mathfrak{X})$ et si l'on pose $y=\Delta(x)$, on a $\operatorname{pr}_{1}(y)=p r_{2}(y)=x$, et par définition de $H$ on a $y \in \operatorname{dom}(H)$.

Prouvons que $\Delta^{-1}(\operatorname{dom}(H)) \subset \operatorname{dom}(F)$. Le S-morphisme $p r_{1}$ : $\operatorname{omb}\left(\mathfrak{X} \times_{S} \mathfrak{X}\right) \longrightarrow \operatorname{omb}(\mathfrak{X})$ étant fidèlement plat, et le diagramme suivant commutatif

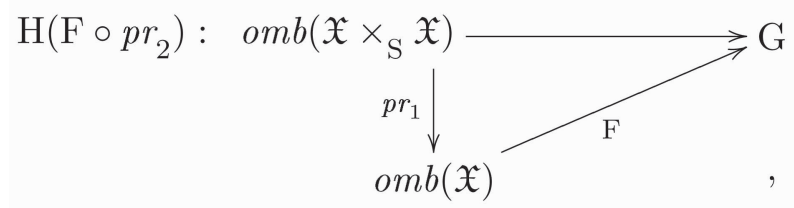

$F$ admet alors un prolognement à $x \in$ omb $(\mathfrak{X})$ si et seulement si la fibre de la restrction $p r_{1} \mid \operatorname{dom}\left(H\left(F \circ p r_{2}\right)\right)$ en $x$ est non-vide.

Soit $x \in \operatorname{omb}(\mathfrak{X})$ tel que $\Delta(x) \in \operatorname{dom}(H)$. Soit q l'idéal premier de $V[[T]]$ correspondant à $x$. Posons $\overline{\mathfrak{q}}=(\mathfrak{q} V[[T]]) \widehat{\otimes}_{V} V[[T]]$. Il est clair que $\overline{\mathfrak{q}}$ est premier. Soit y le point de omb $\left(\mathfrak{X} \times_{S} \mathfrak{X}\right)$ donné par $\bar{q}$. On vérifie que $\operatorname{pr}_{2}(y) \in \operatorname{dom}(F)$ car le point générique d'une fibre de omb $(\mathfrak{X}) \longrightarrow S$ appartient toujours à dom $(F)$. D'autre part on a que $y \in d o m(H)$, car y est une générisation de $\Delta(x)$. Il en résulte qu'au point y les morphismes $H$ et $F \circ p r_{2}$ sont définis. Ainsi la fibre de $p r_{1} \mid \operatorname{dom}\left(H\left(F \circ p r_{2}\right)\right)$ en $x$ est nonvide, d'où $x \in \operatorname{dom}(F)$.

C.Q.F.D.

Preuve. de (4.1.15). Soit $U$ un ouvert affine de $G$ voisinage de l'image de $S$ par la section identité $e_{G}$ de $G$. Cet ouvert existe car $S$ est local. Le $S$-morphisme rationnel $H: \operatorname{omb}\left(\mathfrak{X} \times_{S} \mathfrak{X}\right) \longrightarrow G$ donne lieu à un 
S-morphisme rationnel

$$
H^{\prime}: \operatorname{omb}\left(\mathfrak{X} \times_{S} \mathfrak{X}\right) \longrightarrow U .
$$

En plus on $a$

$$
\Delta^{-1}\left(\operatorname{dom}\left(H^{\prime}\right)\right)=\Delta^{-1}(\operatorname{dom}(H)) .
$$

En effet si $H$ admet un prolongement à $\Delta(x)(x \in$ omb(x)) on a nécessairement $H(\Delta(x))=e_{G}\left(x^{\prime}\right)$, où $x^{\prime}$ désigne l'image de $x$ par omb $(\mathfrak{X}) \longrightarrow S, \operatorname{car} G$ est séparé sur $S$.

D'autre part omb $\left(\mathfrak{X} \times_{S} \mathfrak{X}\right)$ est une schéma régulier, donc normal. L'ouvert $U$ étant affine, le complémentaire omb $\left(\mathfrak{X} \times_{S} \mathfrak{X}\right)-d o m\left(H^{\prime}\right)$ de $\operatorname{dom}\left(H^{\prime}\right)$ dans omb $\left(\mathfrak{X} \times_{S} \mathfrak{X}\right)$ est une réunion de composantes irréductibles de codimension 1 dans omb $\left(\mathfrak{X} \times_{S} \mathfrak{X}\right)$. Soit $Z$ une telle composante. Il existe $f \in V[[T]] \widehat{\otimes}_{V} V[[T]]$ tel que $Z=V(f)$. Soit f' l'image de $f$ par

$$
V[[T]] \otimes_{V} V[[T]] \longrightarrow \frac{V[[T]] \otimes_{V} V[[T]]}{(T \otimes 1-1 \otimes T)} \simeq V[[T]]
$$

On a alors

$$
\Delta^{-1}(Z)=V\left(f^{\prime}\right)
$$

Comme $V[[T]]$ est un anneau régulier alors $V\left(f^{\prime}\right)$ est soit une réunion de composantes irréductibles de codimension 1 soit vide. Par (4.1.16) on a

$$
\operatorname{omb}(\mathfrak{X})-\Delta^{-1}\left(\operatorname{dom}\left(H^{\prime}\right)\right)=\operatorname{omb}(\mathfrak{X})-\Delta^{-1}(\operatorname{dom}(H))=\operatorname{omb}(\mathfrak{X})-\operatorname{dom}(F)
$$

donc on trouve que omb(x) - $\operatorname{dom}(F)$ est soit une réunion de composantes irréductibles de codimension 1 soit vide. Par hypothèse on a alors $\operatorname{omb}(\mathfrak{X})-\operatorname{dom}(F)=\varnothing, \quad$ c.à.d. $F$ admet un prolongement $\grave{a}$ $\operatorname{omb}(\mathfrak{X})$.

C.Q.F.D.

Corollaire 4.1.17. Soit $\alpha \in G(\mathfrak{H})$ tel que $\alpha_{\{K\}} \in G\left(o m b\left(\mathfrak{X}_{K}\right)\right)$. On a alors que $\alpha \in G(o m b(\mathfrak{X}))$.

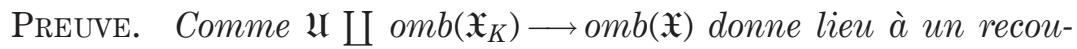
vrement fpqc de omb $(\mathfrak{X})-\{x\}=\mathfrak{U} \cup \operatorname{omb}\left(\mathfrak{X}_{K}\right)$. On a alors que $\alpha$ admet $u n$ prolongement à omb $\mathfrak{x})-\{x\}$, et on conclut par (4.1.15).

C.Q.F.D.

Corollaire 4.1.18. Soit $\alpha \in G(\mathfrak{U})$. Il existe un entier $n>0$ tel que :

$$
\operatorname{tr}_{\theta_{n^{\prime}}} \alpha \equiv \operatorname{tr}_{\theta_{n^{\prime \prime}}} \alpha(\bmod G(\operatorname{omb}(\mathfrak{X})))
$$

si $n^{\prime}, n^{\prime \prime} \geq n$ sont des entiers impairs. 
Preuve. Par (4.1.12) il existe un entier $n$ tel que

$$
\operatorname{tr}_{\theta_{n^{\prime}}} \alpha_{\{K\}} \equiv \operatorname{tr}_{\theta_{n^{\prime \prime}}} \alpha_{\{K\}}\left(\bmod G_{K}\left(\operatorname{omb}\left(\mathfrak{X}_{K}\right)\right)\right)
$$

si $n^{\prime}, n^{\prime \prime} \geq n$ sont impairs. La restriction de

$$
\beta=\left(t r_{\theta_{n^{\prime}}} \alpha\right)\left(\operatorname{tr}_{\theta_{n^{\prime \prime}}} \alpha\right)^{-1} \in G(\mathfrak{U})
$$

$\grave{a} \mathfrak{U}_{\{K\}}$ admet un prolongement à omb $\left(\mathfrak{X}_{K}\right)$ en vertu de (4.1.12).

On conclut que $\beta \in G($ omb(x)) par (4.1.17).

C.Q.F.D.

REMARQUE 4.1.19. (i) - Soit $\varphi_{\mathfrak{C}}^{0} \in \mathfrak{J}^{0}(\mathfrak{H})$ la section définie dans (1.3.25). On peut alors écrire

$$
\varphi_{\mathfrak{T}}^{0}=(\mathfrak{C}-T)^{-1}
$$

La démonstration de (4.1.11) donne

$$
\operatorname{tr}_{\Theta_{n}} \varphi_{\mathfrak{C}}^{0}=(-1)^{n+1}\left(\mathbb{T}-T^{n}\right)^{-1}
$$

(ii) - Soit $\alpha \in G(\mathfrak{U})$ vérifiant la condition (4.1.4), $\widehat{\alpha}=f \circ \widehat{\varphi}_{T}^{0}$. Soit $\bar{\alpha}=f \circ \widehat{\varphi}_{\mathfrak{C}}^{0}$. On a alors un diagramme commutatif:

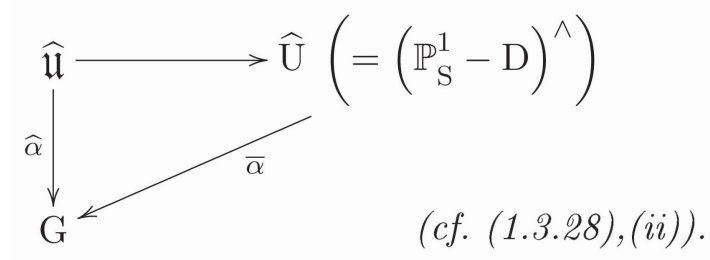

LEMME 4.1.20. Soit $\alpha \in G(\mathfrak{U})$ comme dans (ii) ci-dessus. Il existe alors un entier $n>0$ tel que $\operatorname{tr}_{\theta_{n^{\prime}}} \alpha=\operatorname{tr}_{\theta_{n^{\prime \prime}}} \alpha$, si $n^{\prime}, n^{\prime \prime} \geq n$ sont des entiers impairs.

PREUVE. Montrons que l'entier $n$ obtenu à partir de $\alpha$ par (4.1.18) vérifie la condition de l'énoncé. Il résulte de (i), (4.1.9) que pour tout couple d'entiers impairs $n^{\prime}, n^{\prime \prime}$ on a

(*) $\quad\left(\operatorname{tr}_{\Theta_{n^{\prime}}} \varphi_{\mathfrak{C}}^{0}\right)\left(\operatorname{tr}_{\Theta_{n^{\prime \prime}}} \varphi_{\mathfrak{T}}^{0}\right)^{-1}=\left(1-\widetilde{T} T^{n^{\prime}}\right)^{-1}\left(1-\mathbb{T}^{-1} T^{n^{\prime \prime}}\right)$.

D'où il résulte que $\left(\operatorname{tr}_{\Theta_{n^{\prime}}} \varphi_{\mathfrak{C}}^{0}\right)\left(\operatorname{tr}_{\Theta_{n^{\prime \prime}}} \varphi_{\mathfrak{C}}^{0}\right)^{-1}$ admet un prolognement à $\mathrm{P}_{S}^{1}-D_{(0)}$. Par définition de $\bar{\alpha}$ on a, pour tout entier $v>0$, l'égalité 
suivante

$$
\left(\operatorname{tr}_{\Theta_{n^{\prime}}} \bar{\alpha}^{(v)}\right)\left(t r_{\Theta_{n^{\prime \prime}}} \bar{\alpha}^{(v)}\right)^{-1}=f^{(v)}\left(\left(\left(\operatorname{tr}_{\Theta_{n^{\prime}}} \varphi_{\mathfrak{C}}^{0}\right)\left(t r_{\Theta_{n^{\prime \prime}}} \varphi_{\mathbb{C}}^{0}\right)^{-1}\right)^{(v)}\right)
$$

Si $n^{\prime}, n^{\prime \prime}>n$ la section

$$
\beta^{(v)}=\left(\operatorname{tr}_{\Theta_{n^{\prime}}} \bar{\alpha}^{(v)}\right)\left(\operatorname{tr}_{\Theta_{n^{\prime \prime}}} \bar{\alpha}^{(v)}\right)^{-1}
$$

de $G^{(v)}$ au-dessus de $U^{(v)}=\left(\mathbb{P}_{S}^{1}\right)^{(v)}-D_{(0)}^{(v)}$, admet un prolongement à $\left(\mathbb{P}_{S}^{1}\right)^{(v)}$ car la restriction

$$
\beta^{(v)} \mid \mathfrak{U}^{(v)}=\left(\operatorname{tr}_{\theta_{n^{\prime}}} \alpha^{(v)}\right)\left(\operatorname{tr}_{\theta_{n^{\prime \prime}}} \alpha^{(v)}\right)^{-1}
$$

admet un prolongement à omb $(\mathfrak{X})^{(v)}$. On a ainsi un $S^{(v)}$-morphisme de la droite projective $\mathbb{P}_{S^{(v)}}^{1}$ dans $G^{(v)}$ qui est alors constant (cf. Exp. XVIII, [7]). D'autre part d'après $(*)$ il résulte que la restriction de $\beta^{(v)} \grave{a} D_{(\infty)}^{(v)} \subset\left(\mathbb{P}_{S}^{1}\right)^{(v)}$ coïncide avec la section identité de $G^{(v)}$ au-dessus de $D_{(\infty)}^{(v)}$. On en déduit pour $n^{\prime}, n^{\prime \prime}>n$ que pour tout entier $v>0$ on $a$

$$
\operatorname{tr}_{\Theta_{n^{\prime}}} \bar{\alpha}^{(v)}=\operatorname{tr}_{\Theta_{n^{\prime \prime}}} \bar{\alpha}^{(v)}
$$

et à fortiori

$$
\operatorname{tr}_{\theta_{n^{\prime}}} \alpha^{(v)}=\operatorname{tr}_{\theta_{n^{\prime \prime}}} \alpha^{(v)} \text { d'où } \operatorname{tr}_{\theta_{n^{\prime}}} \alpha=\operatorname{tr}_{\theta_{n^{\prime \prime}}} \alpha
$$

C.Q.F.D.

Preuve. de la Proposition (4.1.6).

Soit $n$ un entier comme dans (4.1.20), et $\ell>n$ un entier impair. Si $v \in \mathbb{N}$ il existe $n(v) \in \mathbb{N}$ pair tel que $n(v)>n$ et $f^{(v)}$ se factorise par $\left.\left(\mathfrak{J}^{0}\right)^{(v)} \longrightarrow\left(\mathfrak{J}_{n(v)}^{0}\right)\right)^{(v)}$. D'après (4.1.20) on a $\operatorname{tr}_{\theta_{\ell}} \alpha=\operatorname{tr}_{\theta_{n(v)+1}} \alpha$, d'où $\operatorname{tr}_{\theta_{\ell}} \alpha^{(v)}=$ $\operatorname{tr}_{\theta_{n(v)+1}} \alpha^{(v)}$. D'autre part, par (4.1.11), on a, pour tout $v>0$,

$$
\operatorname{tr}_{\theta_{n(v)+1}} \alpha^{(v)}={ }^{m} \alpha^{(v)}
$$

d'où $\left(\operatorname{tr}_{\theta_{\ell}} \alpha\right)^{(v)}=\operatorname{tr}_{\theta_{\ell}} \alpha^{(v)}={ }^{m} \alpha^{(v)}$, donc ${ }^{m} \widehat{\alpha}$ est algébrisable. En fait ${ }^{m} \widehat{\alpha}$ est la fibre formelle de ${ }^{m} \alpha=\operatorname{tr}_{\theta_{\ell}} \alpha$.

C.Q.F.D.

Posons

$$
\varphi_{\mathfrak{\mathbb { C }}}^{0}={ }^{m} \varphi_{\mathfrak{\mathbb { C }}}^{0} \varphi^{u} \varphi_{\mathfrak{\mathbb { C }}}^{0},
$$

où ${ }^{m} \varphi_{\mathbb{E}}^{0}: U \longrightarrow \underline{\mathrm{G}}_{m S}\left(\right.$ resp. $\left.{ }^{u} \varphi_{\mathfrak{C}}^{0}: U \longrightarrow \wedge^{0}\right)$ est donnée par la section de $\underline{\mathrm{G}}_{m S}$ au-dessus de $U$, donnée par la restriction de $\mathfrak{T}^{-1}$ à $U$ (resp. de $\wedge^{0}$ 
au-dessus de $U$ donnée par la section $\left(1-\mathbb{T}^{-1} T\right)^{-1}$ ) (cf. (4.1.1)). Avec les notations de (1.3) et celles ci-dessus on a le diagramme commutatif suivant

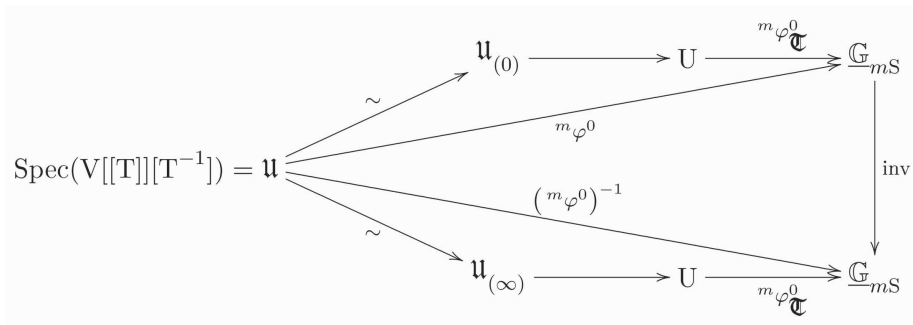

Le $S$-morphisme formel

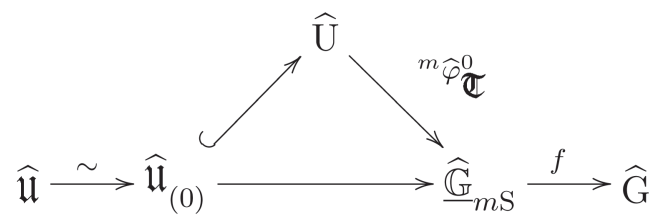

est égal à la fibre formelle ${ }^{m} \widehat{\alpha}$ de ${ }^{m} \alpha$. D'autre part il résulte d'après (4.1.22) que le morphisme composé

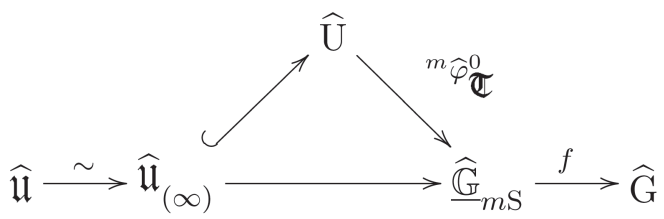

est égal à

$$
f \circ\left({ }^{m} \widehat{\varphi}^{0}\right)^{-1}=\left({ }^{m} \widehat{\alpha}\right)^{-1},
$$

d'où il résulte qu'il est égal à la fibre formelle de ${ }^{m} \alpha^{-1}$, donc qu'il est algébrisable.

DÉFINITION 4.1.23. On pose

$$
{ }^{m} \bar{\alpha}=f \circ{ }^{m} \widehat{\varphi}_{\mathbb{C}}^{0}\left(\operatorname{resp} .{ }^{u} \bar{\alpha}=f \circ{ }^{u} \widehat{\varphi}_{\mathfrak{C}}^{0}\right),
$$

d'où

$$
\bar{\alpha}={ }^{m} \bar{\alpha}^{u} \bar{\alpha} .
$$


On a alors le diagramme commutatif

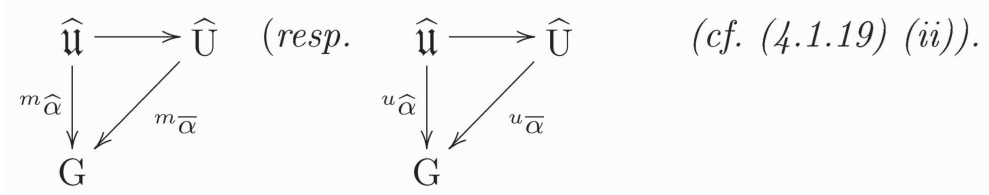

Notons que ${ }^{u} \bar{\alpha}: \widehat{\mathfrak{U}} \longrightarrow G$ admet un prolongement à $\widehat{U}_{(\infty)}$, où $U_{(\infty)}=\mathbb{P}_{S}^{1}-D_{(0)}$ (cf. (1.3.4)). En effet ${ }^{u} \bar{\alpha}=f \circ^{u} \widehat{\varphi}_{\mathfrak{C}}^{0}$, et ${ }^{u} \varphi_{\mathfrak{\mathbb { C }}}^{0}$ est donné par la section $\left(1-\mathbb{C}^{-1} T\right)$ de $\wedge^{0}$ au-dessus de $U$, qui admet un prolongement évident à $U_{(\infty)}$. Ce qui suffit à prouver que $\operatorname{omb}\left({ }^{u} \bar{\alpha}\right)$ composé avec $\operatorname{omb}\left(\widehat{\mathfrak{U}}_{(\infty)}\right) \longrightarrow \operatorname{omb}(\widehat{U})$ se factorise par un morphisme omb $\left(\mathfrak{X}_{(\infty)}\right) \longrightarrow G$, et à fortiori par $\mathfrak{H}_{(\infty)} \longrightarrow G$.

On a alors la

Proposition 4.1.25. Le morphisme

$$
m_{\bar{\alpha}}: \widehat{U} \longrightarrow \widehat{G}
$$

vérifie la condition suivante : la restriction de ${ }^{m} \bar{\alpha} \grave{a} \widehat{\mathfrak{U}}_{(0)}$ (resp. $\widehat{U}_{(\infty)}$ ) s'obtient par passage à la fibre formelle à partir du morphisme

$$
\operatorname{tr}_{\theta_{n}} \alpha: \mathfrak{U} \longrightarrow G\left(\operatorname{resp} .\left(\operatorname{tr}_{\theta_{n}} \alpha\right)^{-1}: \mathfrak{U} \longrightarrow G\right)
$$

pour un entier $n$ convenable.

Il s'agit maintenant de montrer, à partir de (4.1.25), que le morphisme ${ }^{m} \bar{\alpha}$ (resp. $\left.{ }^{u} \bar{\alpha}\right)$ provient, par passage à la fibre formelle, d'un $S$-morphisme $W \longrightarrow G$, où $W$ désigne un voisinage ouvert de la fibre spéciale $U_{k}$ de $U$.

Le $S$-schéma $G$ étant séparé et de présentation finie, on a alors un $S$ morphisme de schémas

$$
\operatorname{omb}\left({ }^{m} \bar{\alpha}\right)\left(\operatorname{resp} . \operatorname{omb}\left({ }^{u} \bar{\alpha}\right)\right): \operatorname{omb}(\widehat{U}) \longrightarrow G
$$

induisant ${ }^{m} \widehat{\alpha}$ (resp. $\left.{ }^{n} \widehat{\alpha}\right)$ par restriction à $\widehat{\mathfrak{U}} \longrightarrow \operatorname{omb}(\widehat{U})$. D'autre part la fibre générique $G_{K}$ de $G$ est un $K$-schéma en groupes lisse et connexe, et l'on sait alors que $G_{K}$ est une $K$-variété quasi-projective. Il existe alors une immersion dans un $K$-espace projectif

$$
i: G_{K} \longrightarrow \mathbb{P}_{K}^{N} .
$$

Corollaire 4.1.28. Le K-morphisme

$$
i \circ o m b\left({ }^{m} \bar{\alpha}\right)_{K}\left(\operatorname{resp} . i \circ \mathrm{omb}\left({ }^{u} \bar{\alpha}\right)_{K}\right): \operatorname{omb}(\widehat{U})_{K} \longrightarrow \mathbb{P}_{K}^{N}
$$


obtenu en composant la fibre générique omb $\left({ }^{m} \bar{\alpha}\right)_{K}\left(\operatorname{resp} . \operatorname{omb}\left({ }^{u} \bar{\alpha}\right)_{K}\right) d e$ (4.1.26) avec i, vérifie les conditions :

a) - La composition de $i \circ o m b\left({ }^{m} \bar{\alpha}\right)_{K}\left(\right.$ resp. $\left.i \circ o m b\left({ }^{u} \bar{\alpha}\right)_{K}\right)$ avec $\operatorname{omb}\left(\widehat{\mathfrak{U}}_{(0)}\right)_{K} \longrightarrow \operatorname{omb}(\widehat{U})_{K}$ provient d'un $K$-morphisme $\quad \mathfrak{U}_{(0)_{K}} \longrightarrow \mathbb{P}_{K}^{N}$ par composition avec omb $\left(\widehat{\mathfrak{U}}_{(0)}\right)_{K} \longrightarrow \mathfrak{U}_{(0) K}$.

b) - La composition de $i \circ o m b\left({ }^{m} \bar{\alpha}\right)_{K}\left(r e s p . i \circ o m b\left({ }^{u} \bar{\alpha}\right)_{K}\right)$ avec $\operatorname{omb}\left(\widehat{\mathfrak{U}}_{(\infty)}\right)_{K} \longrightarrow \operatorname{omb}(\widehat{U})_{K}$ provient d'un K-morphisme $\mathfrak{U}_{(\infty)_{K}} \longrightarrow \mathbb{P}_{K}^{N}$ par composition avec omb $\left(\widehat{\mathfrak{U}}_{(\infty)}\right)_{K} \longrightarrow \mathfrak{U}_{(\infty) K}$. Notons que ${ }^{u} \bar{\alpha}: \widehat{U} \longrightarrow$ G admet un prolongement à $\widehat{U}_{(\infty)}$, ò̀ $U_{(\infty)}=\mathbb{P}_{S}^{1}-D_{(0)}$ (cf. (1.3.4)). En effet ${ }^{u} \bar{\alpha}=f \circ^{u} \widehat{\varphi}_{\mathfrak{C}}^{0}$ et ${ }^{u} \varphi_{\mathfrak{\mathbb { C }}}^{0}$ est donné par la section $\left(1-\mathbb{T}^{-1} T\right)^{-1}$ de $\wedge^{0}$ au-dessus de $U$, qui admet un prolongement évident à $U_{(\infty)}$. Ce qui suffit à prouver que omb $\left({ }^{u} \bar{\alpha}\right)$ composé avec omb $\left(\widehat{\mathfrak{U}}_{(\infty)}\right) \longrightarrow \operatorname{omb}(\widehat{U})$ se factorise par un morphisme omb $\left(\mathfrak{X}_{(\infty)}\right) \longrightarrow G$.

(4.2) Le but de ce numéro est de prouver le

LEMME D'ALGÉBRISATION (4.2.1). On reprend les notations du numéro précédent. Soit

$$
\psi: \operatorname{omb}(\widehat{U})_{K} \longrightarrow \mathbb{P}_{K}^{N}
$$

un $K$-morphisme tel qu'il existe un diagramme commutatif :

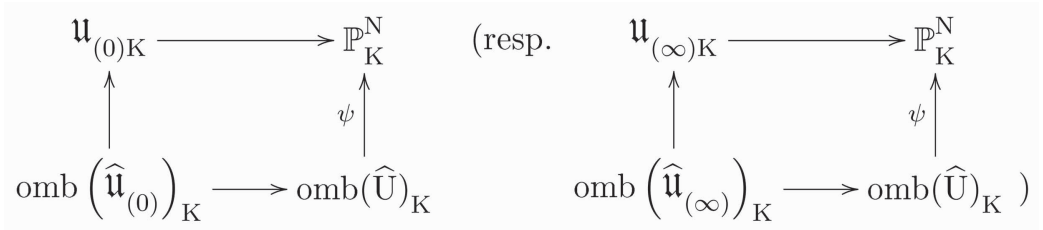

Alors in existe un $K$-morphisme

$$
\bar{\psi}: U_{K} \longrightarrow \mathbb{P}_{K}^{N}
$$

et un diagramme commutatif

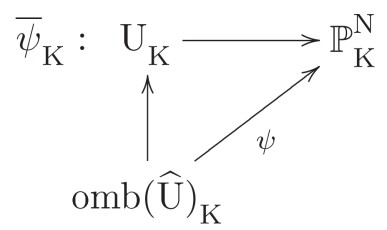


Soient $V[T]^{\wedge}$ (resp. $V\left[T^{-1}\right]^{\wedge}$ ) le complété formel de $V[T]$ (resp. $\left.V\left[T^{-1}\right]\right)$ pour la topologie $m$-adique, et $V\left[T, T^{-1}\right]^{\wedge}$ le complété formel de $V\left[T, T^{-1}\right]$ pour la topologie $m$-adique. Noter que

$$
\widehat{U}=\operatorname{Spf}\left(V\left[T, T^{-1}\right]^{\wedge}\right),
$$

d'où

$$
\operatorname{omb}(\widehat{U})=\operatorname{Spec}\left(V\left[T, T^{-1}\right]^{\wedge}\right)
$$

D'autre part on a

$$
\widehat{U}_{(0)}=\operatorname{Spf}\left(V[T]^{\wedge}\right) \quad\left(\text { resp. } \widehat{U}_{(\infty)}=\operatorname{Spf}\left(V\left[T^{-1}\right]^{\wedge}\right)\right)
$$

et

(4.2.5) $\operatorname{omb}\left(\widehat{U}_{(0)}\right)=\operatorname{Spec}\left(V[T]^{\wedge}\right) \quad\left(\operatorname{resp.} \operatorname{omb}\left(\widehat{U}_{(\infty)}\right)=\operatorname{Spec}\left(V\left[T^{-1}\right]^{\wedge}\right)\right)$

L'anneau $V[T]^{\wedge}$ est un anneau de Zariski et comme

$$
\left.V\left[T, T^{-1}\right]^{\wedge}=V[T]_{\{T\}}^{\wedge} \quad \text { (localisé complété de } V[T]^{\wedge}\right)
$$

il en résulte que le morphisme

$$
\begin{gathered}
\operatorname{omb}(\widehat{U}) \coprod \operatorname{omb}\left(\mathfrak{x}_{(0)}\right) \longrightarrow \operatorname{omb}\left(\widehat{U}_{(0)}\right) \\
\left(\operatorname{resp.} \operatorname{omb}(\widehat{U}) \coprod \operatorname{omb}\left(\mathfrak{X}_{(\infty)}\right) \longrightarrow \operatorname{omb}\left(\widehat{U}_{(\infty)}\right)\right)
\end{gathered}
$$

est fidèlement plat.

L'anneau $V\left[[T]\left[T^{-1}\right]^{\wedge}\right.$ obtenu comme le complété formel de $V[[T]]\left[T^{-1}\right]$ pour la topologie $m$-adique est un anneau de valuation discrète de corps résiduel $k[[T]]\left[T^{-1}\right]$. Le critère valuatif de propreté donne alors le

LEMME 4.2.8. Avec les notations de (4.2.1). Il existe un diagramme commutatif:
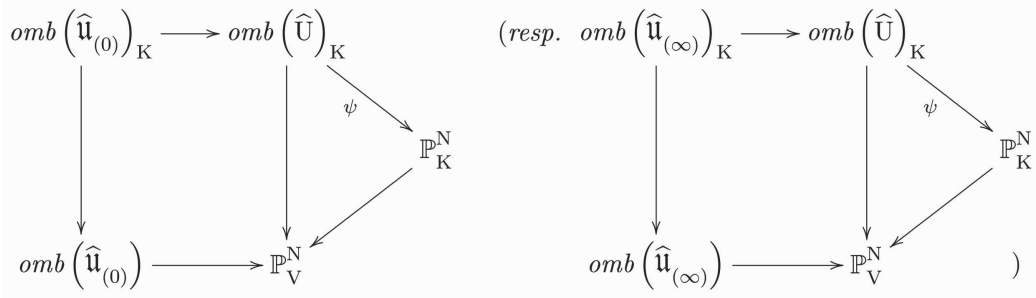
Noter que $\operatorname{omb}\left(\mathfrak{X}_{(0)}\right)$ (resp. omb( $\left.\mathfrak{X}_{(\infty)}\right)$ ) est un schéma local. Soit $x_{(0)} \in \operatorname{omb}\left(\mathfrak{X}_{(0)}\right)\left(\operatorname{resp} . x_{(\infty)} \in \operatorname{omb}\left(\mathfrak{X}_{(\infty)}\right)\right)$ le point fermé. Le $S$-morphisme

(4.2.9) $\operatorname{omb}\left(\widehat{\mathfrak{U}}_{(0)}\right) \coprod \mathfrak{U}_{(0) K} \coprod \operatorname{omb}\left(\mathfrak{X}_{(0) K}\right) \longrightarrow \operatorname{omb}\left(\mathfrak{X}_{(0)}\right)-\left\{x_{(0)}\right\}$

$$
\left(\operatorname{resp} . \operatorname{omb}\left(\widehat{\mathfrak{U}}_{(\infty)}\right) \coprod \mathfrak{U}_{(\infty) K} \coprod \operatorname{omb}\left(\mathfrak{X}_{(\infty) K}\right) \longrightarrow \operatorname{omb}\left(\mathfrak{X}_{(\infty)}\right)-\left\{x_{(\infty)}\right\}\right)
$$

est fidèlement plat. D'autre part le morphisme

$$
\mathfrak{U}_{(0) K} \longrightarrow \mathbb{P}_{K}^{N}\left(\text { resp. } \mathfrak{H}_{(\infty)} \longrightarrow \mathbb{P}_{K}^{N}\right),
$$

donné par l'hypothèse de (4.2.1), composé avec

$$
\begin{gathered}
\mathfrak{U}_{(0)\{K\}}=\operatorname{Spec}\left(K[[T]]\left[T^{-1}\right]\right) \longrightarrow \mathfrak{U}_{(0) K} \\
\left(\text { resp. } \mathfrak{H}_{(\infty)\{K\}}=\operatorname{Spec}\left(K\left[\left[T^{-1}\right]\right][T]\right) \longrightarrow \mathfrak{U}_{(\infty) K}\right)
\end{gathered}
$$

admet un prolongement

$$
\operatorname{omb}\left(\mathfrak{X}_{(0) K}\right) \longrightarrow \mathbb{P}_{K}^{N}\left(\operatorname{resp} . \operatorname{omb}\left(\mathfrak{X}_{(\infty) K}\right) \longrightarrow \mathbb{P}_{K}^{N}\right)
$$

en vertu du critère valuatif de propreté. Grâce au morphisme fidèlement plat (4.2.9) et à partir des morphismes donnés par (4.2.8), (4.2.10) et (4.2.11) on démontre le

Lemme 4.2.12. Avec les notations de (4.2.1). Il existe un diagramme commutatif

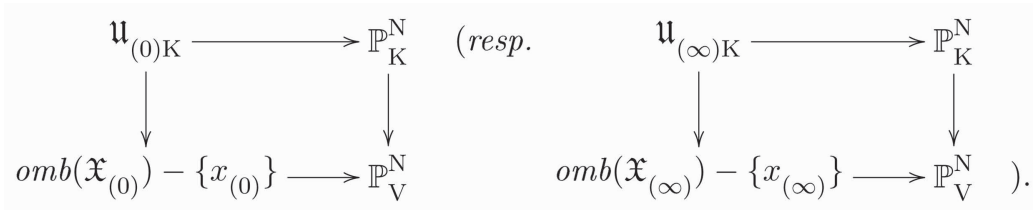

Il existe un éclatement (cf. [12])

$$
\mathfrak{Z}_{(0)} \longrightarrow \operatorname{omb}\left(\mathfrak{X}_{(0)}\right)\left(\operatorname{resp} . \mathfrak{Z}_{(\infty)} \longrightarrow \operatorname{omb}\left(\mathfrak{X}_{(\infty)}\right)\right)
$$

de centre un sous-schéma fermé $\tau_{(0)}\left(\right.$ resp. $\left.\tau_{(\infty)}\right)$ de support $x_{(0)}\left(\operatorname{resp} . x_{(\infty)}\right)$, et un diagramme commutatif

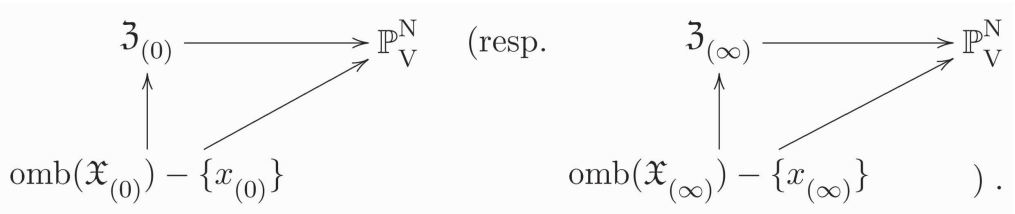


D'autre part il résulte du critère valuatif de propreté qu'il existe un diviseur $\delta$ de la fibre spéciale omb $(\widehat{U})_{k}=U_{k}$ et un diagramme commutatif :

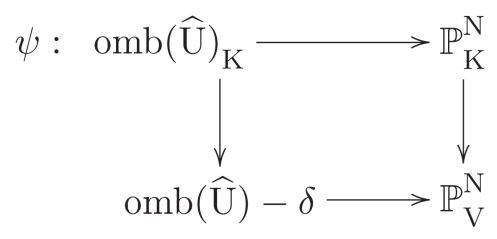

On peut alors trouver un éclatement

$$
Y \longrightarrow \mathbb{P}_{V}^{1}
$$

ayant pour centre un sous-schéma fermé $T$ de $\mathbb{P}_{V}^{1}$ de même support que $\delta$, tel que si l'on pose $\operatorname{omb}(\widehat{U})^{\prime}=\operatorname{omb}(\widehat{U}) \times_{\mathbb{P}_{V}^{1}} Y$ on a les diagrammes commutatifs

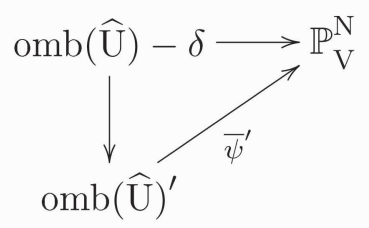

(cf. [12])

et

(4.2.17 bis)

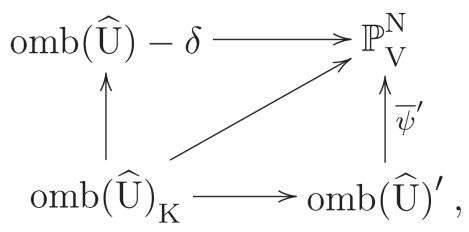

où la flèche diagonale est égale au morphisme composé de $\psi$ avec $\mathbb{P}_{K}^{N} \hookrightarrow \mathbb{P}_{V}^{N}$.

REMARqUe 4.2.18. Il est clair que omb $(\widehat{U})^{\prime}$ s'identifie à l'éclatement de $\operatorname{omb}(\widehat{U})$ de centre le sous-schéma omb $(\widehat{U}) \times_{\mathbb{P}_{V}^{1}} T$. En effet, soient $A$ un anneau, $A \longrightarrow A^{\prime}$ une extension fidèlement plate, et $q \subset A$ un idéal.

Posons

$$
\begin{gathered}
X=\operatorname{Spec}(A)\left(\operatorname{resp} . X^{\prime}=\operatorname{Spec}\left(A^{\prime}\right), q^{\prime}=q A^{\prime},\right. \\
\left.\tilde{X}=\operatorname{Proj}\left(A \oplus q \oplus q^{2} \ldots\right), \tilde{X}^{\prime}=\operatorname{Proj}\left(A^{\prime} \oplus q^{\prime} \oplus q^{\prime 2} \oplus \ldots\right)\right) .
\end{gathered}
$$

On a alors

$$
\tilde{X}^{\prime} \simeq \tilde{X} \times_{A} X^{\prime}
$$


Il en résulte, si l'on pose $U^{\prime}=U \times_{\mathbb{P}_{V}^{1}} Y$, que le complété formel $\widehat{U}^{\prime}$ de $U^{\prime}$, pour la topologie m-adique, s'identifie canoniquement au complété formel $\left(\operatorname{omb}(\widehat{U})^{\prime}\right)^{\wedge}$, pour la topologie m-adique, de omb $(\widehat{U})^{\prime}$. En effet, le morphisme omb $(\widehat{U}) \longrightarrow U$ est plat, et pour tout entier $v>0$ il induit un isomorphisme des voisinages v-èmes.

$$
\operatorname{omb}(\widehat{U})_{v}^{\prime}=\operatorname{omb}(\widehat{U})_{v} \times_{\mathbb{P}_{V}^{1}} Y \longrightarrow U_{v}^{\prime}=U_{v} \times_{\mathbb{P}_{V}^{1}} Y .
$$

Soit $\bar{T} \subset \mathbb{P}_{V}^{1}$ un sous-schéma fermé ayant son support contenu dans $\delta \cup\left\{x_{(0)}\right\} \cup\left\{x_{(\infty)}\right\}$ tel que :

(i) $U \times_{\mathbb{P}_{V}^{1}} \bar{T}=T$,

(ii) $\operatorname{omb}\left(\mathfrak{X}_{(0)}\right) \times_{P_{V}^{1}} \bar{T}=\tau_{(0)}$,

(iii) $\operatorname{omb}\left(\mathfrak{X}_{(\infty)}\right) \times_{P_{V}^{1}} \bar{T}=\tau_{(\infty)}$.

Soit

$$
Z \longrightarrow \mathbb{P}_{V}^{1}
$$

l'éclatement de $\mathbb{P}_{V}^{1}$ de centre $\bar{T}$. On a alors

$$
Z_{U}=U \times_{\mathbb{P}_{V}^{1}} Z=U \times_{\mathbb{P}_{V}^{1}} Y, \mathfrak{Z}_{(0)}=\operatorname{omb}\left(\mathfrak{X}_{(0)}\right) \times_{\mathbb{P}_{V}^{1}} Z
$$

et

$$
\mathfrak{Z}_{(\infty)}=\operatorname{omb}\left(\mathfrak{X}_{(\infty)}\right) \times_{\mathbb{P}_{V}^{1}} Z \text {. }
$$

Posons

$$
\begin{gathered}
\operatorname{omb}\left(\widehat{U}_{(0)}\right)^{\prime}=\operatorname{omb}\left(\widehat{U}_{(0)}\right) \times_{\mathbb{P}_{V}^{1}} Z \\
\left(\text { resp. omb }\left(\widehat{U}_{(\infty)}\right)^{\prime}=\operatorname{omb}\left(\widehat{U}_{(\infty)}\right) \times_{\mathbb{P}_{V}^{1}} Z\right),
\end{gathered}
$$
et notons par $\left(\operatorname{omb}\left(\widehat{U}_{(0)}\right)^{\prime}\right)^{\wedge}\left(\operatorname{resp} .\left(\operatorname{omb}\left(\widehat{U}_{(\infty)}\right)^{\prime}\right)^{\wedge}\right)$ son complété for-
mel.

Comme le morphisme (4.2.7) est fidèlement plat on en déduit à partir de (4.2.18) et (4.2.7) que le morphisme

$$
\begin{gathered}
\operatorname{omb}(\widehat{U})^{\prime} \coprod \mathfrak{Z}_{(0)} \longrightarrow \operatorname{omb}\left(\widehat{U}_{(0)}\right)^{\prime} \\
\left(\operatorname{resp.} \operatorname{omb}(\widehat{U})^{\prime} \coprod \mathfrak{Z}_{(\infty)} \longrightarrow \operatorname{omb}\left(\widehat{U}_{(\infty)}\right)^{\prime}\right)
\end{gathered}
$$

l'est aussi. On définit un $V$-morphisme

$$
\begin{gathered}
\operatorname{omb}\left(\widehat{U}_{(0)}\right)^{\prime} \longrightarrow \mathbb{P}_{V}^{N} \\
\left(\operatorname{resp.} \operatorname{omb}\left(\widehat{U}_{(\infty)}\right)^{\prime} \longrightarrow \mathbb{P}_{V}^{N}\right)
\end{gathered}
$$


à partir du morphisme omb $(\widehat{U})^{\prime} \longrightarrow \mathbb{P}_{V}^{N}$ donné par (4.2.17), et du morphisme $\mathfrak{Z}_{(0)} \longrightarrow \mathbb{P}_{V}^{N}$ (resp. $\mathfrak{Z}_{(\infty)} \longrightarrow \mathbb{P}_{V}^{N}$ ) donné par (4.2.14), grâce au fait que (4.2.21) est un morphisme fidèlement plat. Soit

$$
U_{(0)}^{\prime}=U_{(0)} \times_{\mathbb{P}_{V}^{1}} Z\left(\text { resp. } U_{(\infty)}^{\prime}=U_{(\infty)} \times_{\mathbb{P}_{V}^{1}} Z\right),
$$

et désignons par $\widehat{U}_{(0)}^{\prime}$ (resp. $\widehat{U}_{(\infty)}^{\prime}$ ) le complété formel de $U_{(0)}^{\prime}$ (resp. $\left.U_{(\infty)}^{\prime}\right)$ pour la topologie $m$-adique.

On a alors l'isomorphisme de $V$-schémas formels

$$
\begin{gathered}
\widehat{U}_{(0)}^{\prime} \simeq\left(\operatorname{omb}\left(\widehat{U}_{(0)}\right)^{\prime}\right)^{\wedge} \\
\left(\operatorname{resp} . \widehat{U}_{(\infty)}^{\prime} \simeq\left(\operatorname{omb}\left(\widehat{U}_{(\infty)}\right)^{\prime}\right)^{\wedge}\right) .
\end{gathered}
$$

Notons que $\widehat{U}_{(0)}^{\prime}$ et $\widehat{U}_{(\infty)}^{\prime}$ sont des ouverts du complété formel $\widehat{Z}$ de $Z$ et que

$$
\widehat{Z}=\widehat{U}_{(0)}^{\prime} \cup \widehat{U}_{(\infty)}^{\prime} \quad\left(\text { resp. } \widehat{U}^{\prime}=\widehat{U}_{(0)}^{\prime} \cap \widehat{U}_{(\infty)}^{\prime}\right) .
$$

D'autre part il est facile de vérifier que l'on a un diagramme commutatif

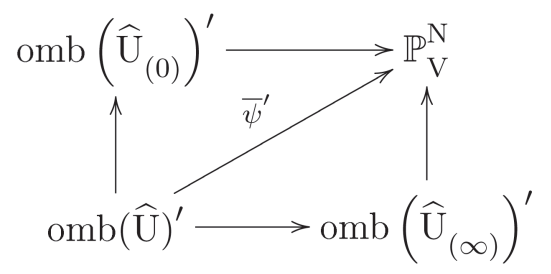

(cf. (4.2.22)).

Il résulte de (4.2.24) que la flèche horizontale supérieure (resp. verticale à droite) de (4.2.26) induit, par passage à la fibre formelle, un morphisme

$$
\widehat{U}_{(0)}^{\prime} \longrightarrow \mathbb{P}_{V}^{N}\left(\text { resp. } \widehat{U}_{(\infty)}^{\prime} \longrightarrow \mathbb{P}_{V}^{N}\right),
$$

tel que le diagramme suivant:

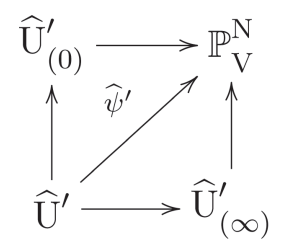


où $\widehat{\psi}^{\prime}$ désigne le morphisme induit par $\bar{\psi}^{\prime}$ (cf. (4.2.24)), commute. On définit ainsi à partir de (4.2.28) (cf. (4.2.25)) un $V$-morphisme formel

$$
\widehat{Z} \longrightarrow \mathbb{P}_{V}^{N}
$$

Le $V$-schéma $Z$ est un $V$-schéma projectif il existe alors un $V$-morphisme

$$
Z \longrightarrow \mathbb{P}_{V}^{N}
$$

redonnant (4.2.29) par passage à la fibre formelle. Notons par $\bar{\psi}: U^{\prime} \longrightarrow \mathbb{P}_{V}^{N}$ la restriction de (4.2.30) à $U^{\prime}$. On a alors le diagramme commutatif

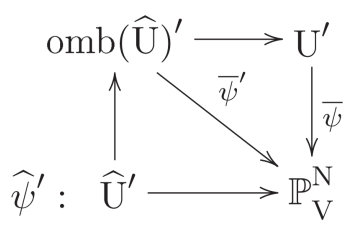

(Le carré commute par définition de $\bar{\psi}$ compte tenu que $\widehat{U}^{\prime} \longrightarrow U^{\prime}$ se factorise par omb $(\widehat{U})^{\prime} \longrightarrow U^{\prime}$; le triangle supérieur (resp. inférieur) commute par définition de $\bar{\psi}^{\prime}$ (resp. comme on le vérifie par restriction de $\bar{\psi}^{\prime}$ à $\left.\widehat{U}^{\prime}\right)$ ). A partir du triangle supérieur de (4.2.31) et du triangle inférieur de (4.2.17) bis on obtient le diagramme commutatif suivant :

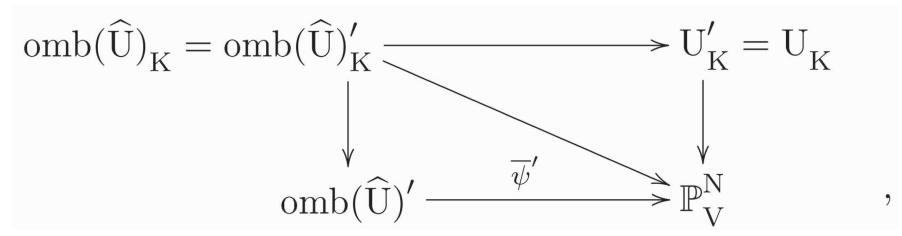

où la flèche diagonale est donnée par la diagonale de (4.2.17) bis, et la flèche verticale droite par le composé de la fibre $\bar{\psi}_{K}: U_{K} \longrightarrow \mathbb{P}_{K}^{N}$ de $\bar{\psi}$ avec $\mathbb{P}_{K}^{N} \longrightarrow \mathbb{P}_{V}^{N}$. On achève de démontrer ainsi (4.2.1).

(4.3) Soit $f: \widehat{\mathfrak{J}}^{0} \longrightarrow \widehat{G}$ défini comme dans (4.1.4). On pose

$$
{ }^{m} f=f \mid \widehat{\widehat{G}}_{m S}\left(\text { resp. }{ }^{u} f=f \mid \widehat{\wedge}^{0}\right) .
$$

On a alors à partir de (4.1.24) que

(4.3.1bis) ${ }^{m} \bar{\alpha}={ }^{m} f \circ{ }^{m} \widehat{\varphi}_{\mathbb{C}}^{0}$ (resp. $\left.{ }^{m} \widehat{\alpha}={ }^{m} f \circ{ }^{m} \widehat{\varphi}_{\mathfrak{C}}^{0},{ }^{u} \bar{\alpha}={ }^{u} f \circ{ }^{u} \widehat{\varphi}_{\mathfrak{C}}^{0},{ }^{u} \widehat{\alpha}={ }^{u} f \circ{ }^{u} \widehat{\varphi}_{\mathfrak{C}}^{0}\right)$. 
Le but de ce numéro est de prouver que ${ }^{m} f$ est algébrisable, c'est-à-dire que l'on a un diagramme commutatif de $S$-groupes

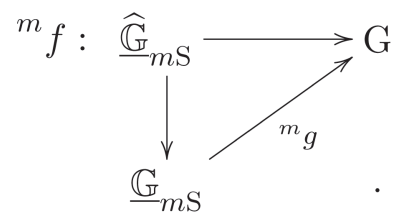

Commençons par prouver que ${ }^{m} \bar{\alpha}$ (resp. ${ }^{u} \bar{\alpha}$ ) est algébrisable. Soit $U_{\text {loc le }}$ localisé de l'ouvert $U$ de $\mathbb{P}_{V}^{N}$ par rapport à l'idéal premier de définition de la fibre spéciale $p=m V\left[T, T^{-1}\right]$, c.à.d.

$$
U_{\text {loc }}=\operatorname{Spec}\left(V\left[T, T^{-1}\right]_{p}\right) .
$$

Noter que le morphisme

$$
\operatorname{omb}(\widehat{U})=\operatorname{Spec}\left(V\left[T, T^{-1}\right]^{\wedge}\right) \longrightarrow U_{\mathrm{loc}}
$$

est fidèlement plat. Le lemme d'algébrisation (cf. (4.2.1)) et (4.1.28), appliqué à

$$
i \circ \operatorname{omb}\left({ }^{m} \bar{\alpha}\right)_{K}\left(\operatorname{resp} . i \circ \operatorname{omb}\left({ }^{u} \bar{\alpha}\right)_{K}\right): \operatorname{omb}(\widehat{U})_{K} \longrightarrow \mathbb{P}_{K}^{N}
$$

donne lieu à un $K$-morphisme $U_{K} \longrightarrow \mathbb{P}_{K}^{N}$ et à un diagramme commutatif

$$
\operatorname{omb}\left({ }^{m} \bar{\alpha}\right)_{\mathrm{K}}\left(\operatorname{resp} \cdot \operatorname{omb}\left({ }^{u} \bar{\alpha}\right)_{\mathrm{K}}\right): \quad \operatorname{omb}(\widehat{\mathrm{U}})_{\mathrm{K}} \longrightarrow \mathrm{G}_{\mathrm{K}} \stackrel{i}{\longrightarrow} \mathbb{P}_{\mathrm{K}}^{\mathrm{N}}
$$

Comme le morphisme $\left.i \circ \operatorname{omb}^{m} \bar{\alpha}\right)_{K}$ (resp. $\left.i \circ \mathrm{omb}\left({ }^{u} \bar{\alpha}\right)_{K}\right)$ se factorise par $i\left(G_{K}\right) \longrightarrow \mathbb{P}_{K}^{N}$, et (4.3.3) est fidèlement plat, on en déduit un diagramme commutatif de $K$-morphismes

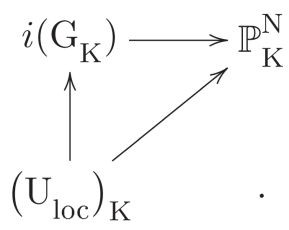

Le morphisme horizontal provient alors d'un unique $K$-morphisme 
$\left(U_{\text {loc }}\right)_{K} \longrightarrow G_{K}$, par composition avec $i$, tel que le diagramme suivant

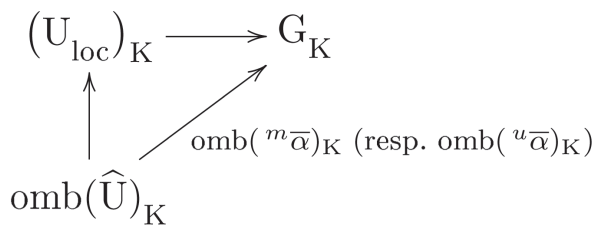

est commutatif. Puisque (4.3.3) est fidèlement plat et $\mathrm{omb}\left({ }^{m} \bar{\alpha}\right)_{K}$ (resp. $\left.\operatorname{omb}\left({ }^{u} \bar{\alpha}\right)_{K}\right)$ admet le prolongement $\operatorname{omb}\left({ }^{m} \bar{\alpha}\right)\left(\operatorname{resp} . o m b\left({ }^{u} \bar{\alpha}\right)\right)$ à omb $(\widehat{U})$ il en résulte un diagramme commutatif

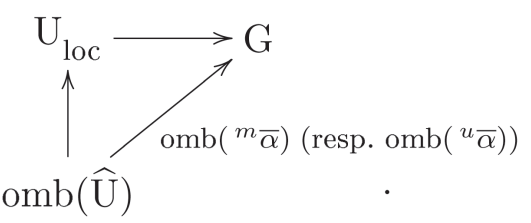

Par un argument de présentation finie on clonclut qu'il existe un voisinage ouvert $W^{\prime}\left(\right.$ resp. $\left.W_{(\infty)}^{\prime}\right)$ de la fibre spéciale $U_{k}$ (resp. $\left.U_{(\infty) k}\right)$, et un $S$-morphisme $W^{\prime}\left(\right.$ resp. $\left.W_{(\infty)}^{\prime}\right) \longrightarrow G$ redonnant $m_{\bar{\alpha}}$ (resp. ${ }^{u} \bar{\alpha}$ ) par passage à la fibre formelle. Soient $W$ (resp. $\left.W_{(\infty)}\right)$ le domaine de définition du $S$-morphisme rationnel donné par $W^{\prime}\left(\right.$ resp. $\left.W_{(\infty)}^{\prime}\right) \longrightarrow G$, et $W\left(\right.$ resp. $\left.W_{(\infty)}\right) \longrightarrow G$ le prolongement de $W^{\prime}\left(\right.$ resp. $\left.W_{(\infty)}^{\prime}\right) \longrightarrow G$ à $W$ $\left(\right.$ resp. $\left.W_{(\infty)}\right)$. On a alors le

LEMME 4.3.8. Il existe un ouvert $W \subset U\left(\right.$ resp. $\left.W_{(\infty)} \subset U_{(\infty)}\right)$ voisinage de la fibre spéciale $U_{k}\left(\right.$ resp. $\left.U_{(\infty) k}\right)$, avec $\mathfrak{H}_{(0)}, \mathfrak{U}_{(\infty)} \subset W\left(\right.$ resp. $\left.W_{(\infty)}\right)$, et un $S$-morphisme $W$ (resp. $\left.W_{(\infty)}\right) \longrightarrow G$ tel que par passage à la fibre formelle donne ${ }^{m} \bar{\alpha}\left(\right.$ resp. $\left.{ }^{u} \bar{\alpha}\right)$.

Preuve. Soit $W$ (resp. $\left.W_{(\infty)}\right)$ comme ci-dessus. Il suffit de prouver que $\mathfrak{U}_{(0)}, \mathfrak{U}_{(\infty)} \subset W$ (resp. $\left.W_{(\infty)}\right)$. Soient $\mathfrak{p} \subset V[[T]]\left[T^{-1}\right]$ un idéal premier, $\mathfrak{q}=\mathfrak{p} \cap V\left[T, T^{-1}\right], U_{\mathfrak{q}}=\operatorname{Spec}\left(V\left[T, T^{-1}\right]_{\mathfrak{q}}\right)$, et $\mathfrak{U}_{(0) \mathfrak{p}}=\operatorname{Spec}\left(V[[T]]\left[T^{-1}\right]_{\mathfrak{p}}\right)$. Voyons que $W$ (resp. $\left.W_{(\infty)}\right) \supset \mathfrak{U}_{(0)}$. Notons que $W \cap U_{\mathfrak{q}} \neq \varnothing$ (resp. $\left.W_{(\infty)} \cap U_{\mathfrak{q}} \neq \varnothing\right)$ car le point générique de $U_{\mathfrak{q}}$ appartient à $W$ $\left(\right.$ resp. $\left.W_{(\infty)}\right)$.

Soient $R(U), R\left(U_{\mathfrak{q}}\right), R\left(\mathfrak{H}_{(0)}\right), R\left(\mathfrak{H}_{(0) \mathfrak{p}}\right)$ et $R\left(\widehat{\mathfrak{U}}_{(0)}\right)$ respectivement le corps de fractions de $U, U_{\mathfrak{q}}, \mathfrak{H}_{(0)}, \mathfrak{U}_{(0) \mathfrak{p}}$, et $\widehat{\mathfrak{U}}_{(0)}$. Comme $\mathfrak{H}_{(0) \mathfrak{p}} \longrightarrow U_{\mathfrak{q}}$ est fidèlement 
plat il suffit de montrer que le diagramme

$(*)$

$$
\begin{array}{cl}
\operatorname{Spec}(\mathrm{R}(\mathrm{U}))=\operatorname{Spec}\left(\mathrm{R}\left(\mathrm{U}_{\mathfrak{q}}\right)\right) \longrightarrow \mathrm{G} \\
\operatorname{Spec}\left(\mathrm{R}\left(\mathfrak{u}_{(0)}\right)\right)=\operatorname{Spec}\left(\mathrm{R}\left(\mathfrak{u}_{(0) \mathfrak{p}}\right)\right) \longrightarrow \overbrace{\alpha\left(\text { resp. }{ }^{u} \alpha\right)} \longrightarrow \mathfrak{u}_{(0)}
\end{array}
$$

commute, où la flèche horizontale supérieure est induite par $W\left(\right.$ resp. $\left.W_{(0)}\right) \longrightarrow G$. Or la commutativité de $(*)$ découle de la commutativité du diagramme obtenu à partir de $(*)$ en posant Spec $\left(R\left(\widehat{\mathfrak{U}}_{(0)}\right)\right)$ à la place de Spec $\left(R\left(\mathfrak{U}_{(0)}\right)\right)$; cette commutativité résultant de (4.3.7), et (4.1.19), (ii), compte tenu que ${ }^{m} \alpha=t_{\theta_{\ell}} \alpha$ et ${ }^{u} \alpha=\alpha\left({ }^{m} \alpha\right)^{-1}$ pour $\ell$ assez grand (cf. preuve de (4.1.6)). On procède à prouver que $\mathfrak{U}_{(\infty)} \subset W$ comme ci-dessus à partir du fait que $\widehat{\mathfrak{U}}_{(\infty)} \longrightarrow U \longrightarrow G$ est algébrisable. $\quad$ C.Q.F.D.

Comme ${ }^{m} \varphi_{\mathfrak{C}}^{0}$ établit un isomorphisme entre $U$ et $\underline{G}_{m S}$, on définit un $S$ morphisme

$$
{ }^{m} \varphi_{\mathfrak{T}}^{0}(W) \longrightarrow G
$$

comme étant le seul qui rend commutatif le diagramme suivant

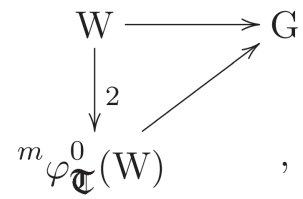

où le morphisme horizontal est donné par (4.3.8). Noter que $\widehat{W}=\widehat{U}$, et $\left({ }^{m} \varphi_{\widetilde{C}}^{0}(W)\right)^{\wedge}=\widehat{\mathbb{G}}_{m S}$, et que le diagramme (4.3.10) donne par passage à la fibre formelle le diagramme

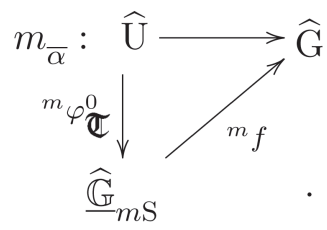

Le morphisme $W \times W \longrightarrow \underline{\mathbb{G}}_{m S}$ composé de ${ }^{m} \varphi_{\mathfrak{C}}^{0} \times{ }^{m} \varphi_{\mathfrak{C}}^{0}$ et la multiplication de $\underline{G}_{m S}$ est fidèlement plat. En effet la platitude est facilement vérifiée et la surjectivité résulte du lemme (0.33), compte tenu que $\mathfrak{U}_{(0)} \subset W$.

Posons $W^{(2)}=W \times W$. Soit $W^{(2) \prime}$ l'image inverse de ${ }^{m} \varphi_{\mathbb{C}}^{0}(W)$ par $W^{(2)} \longrightarrow \underline{G}_{m S}$. Il est clair que le complété formel $\widehat{W}^{(2) \prime}$ de $W^{(2) \prime}$ s'identifie à 
$\widehat{U} \times \widehat{U}$. La commutativité du diagramme suivant

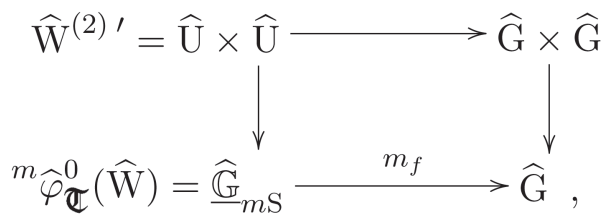

où la flèche horizontale est induite par ${ }^{m} \bar{\alpha} \times^{m} \bar{\alpha}$, et la verticale droite par la multiplication de $G$, entraîne celle de

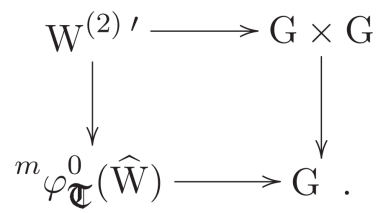

Le morphisme composé $W^{(2) \prime} \longrightarrow G$ admet un prolongement $W^{(2)} \longrightarrow G$, d'où par descente fidèlement plate il résulte que le morphisme ${ }^{m} \varphi_{\widetilde{\tau}}^{0}(W) \longrightarrow G$ admet un prolongement ${ }^{m} g: \underline{G}_{m S} \longrightarrow G$. (Noter que ${ }^{m} \varphi_{\widetilde{C}}^{0}(W)$ est un ouvert schématiquement dense de $\underline{\mathbb{G}}_{m S}$ et que $G$ est séparé). Comme la fibre formelle ${ }^{m} f: \widehat{\mathbb{G}}_{m S} \longrightarrow \widehat{G}$ de ${ }^{m} g: \widehat{G}_{m S} \longrightarrow G$ est un $S$-homomorphisme, il en résulte que ${ }^{m} g$ est un $S$-homomorphisme de groupes qui vérifie

$$
{ }^{m} \alpha={ }^{m} g \circ{ }^{m} \varphi^{0} .
$$

On a alors la

Proposition 4.3.13. Il existe un S-homomorphisme de groupes ${ }^{m} g: \underline{\mathrm{G}}_{m S} \longrightarrow G$ dont la fibre formelle ${ }^{m} \widehat{g}$ coïncide avec ${ }^{m} f$ et tel que ${ }^{m} \alpha={ }^{m} g \circ{ }^{m} \varphi^{0}$.

(4.4) Montrons que ${ }^{u}: \widehat{\wedge}^{0} \longrightarrow \widehat{G}$ provient par passage à la fibre formelle d'un $S$-homomorphisme ${ }^{u} g: \wedge^{0} \longrightarrow G$, c.à.d. qu'il existe un diagramme commutatif de $S$-groupes

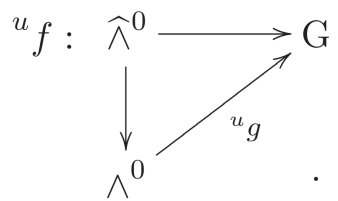


La preuve de ce fait suit les mêmes lignes que celle de (4.3.13). Commençons par prouver la

Proposition 4.4.1. Il existe un entier n, un S-homomorphisme formel ${ }^{u} f_{n}: \widehat{\wedge}_{n}^{0} \longrightarrow \widehat{G}^{0}$ et un diagramme commutatif

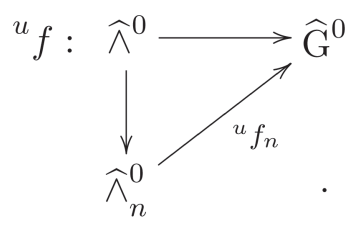

Notons d'abord qu'il résulte de (4.1.11) que

$$
\operatorname{tr}_{\theta_{n}}{ }^{u} \varphi^{0}=\left(1-t^{-1} T^{n}\right)^{-1},
$$

et de la preuve de (4.1.20) qu'il existe $n$, tel que $n^{\prime}, n^{\prime \prime} \geq n$ entraînent que

$$
\operatorname{tr}_{\theta_{n^{\prime}}}{ }^{u} \alpha=\operatorname{tr}_{\theta_{n^{\prime \prime}}}{ }^{u} \alpha
$$

LEMME 4.4.4. On a alors que tr $r_{\theta_{n^{\prime}}}{ }^{u} \alpha$ cö̈ncide avec la section identité de $G$ pour tout entier $n^{\prime} \geq n$.

(La Preuve de (4.4.4) suit celle de (4.1.6).)

Remarque 4.4.5. Posons ${ }^{u} f=\left({ }^{u} f^{(\mu)}\right)$ et ${ }^{u} \widehat{\alpha}=\left({ }^{u} \alpha^{(\mu)}\right)$ et ${ }^{u} \widehat{\varphi}^{0}=$ $\left(\left({ }^{u} \varphi^{0}\right)^{(\mu)}\right)$. Comme on a ${ }^{u} f^{(\mu)} \circ\left({ }^{u} \varphi^{0}\right)^{(\mu)}={ }^{u} \alpha^{(\mu)}$, il en résulte que ${ }^{u} f^{(\mu)}\left(\left(\operatorname{tr}_{\theta_{v}}{ }^{u} \varphi^{0}\right)^{(\mu)}\right)$, pour tout entier $v \geq n$, coïncide avec la section identique de $G^{(\mu)}$ (au-dessus de $\mathfrak{U}^{(\mu)}$ ).

Soient $0 \leq n \leq p$ des entiers. On pose

$$
\wedge_{n p}^{0}=\operatorname{Ker}\left(\wedge_{p}^{0} \longrightarrow \wedge_{n-1}^{0}\right),
$$

et, pour tout $n \leq v \leq p$, on désigne par $\wedge_{n p}^{0}[v]$ le sous-groupe de $\wedge_{n p}^{0}$ dont les sections sont de la forme $1+\sum \alpha_{q} T^{v q}(1 \leq q \leq[p / v])$. On a un $S$-homomorphisme

$$
\prod_{n \leq v \leq p} \wedge_{n p}^{0}[v] \longrightarrow \wedge_{n p}^{0}
$$

donné par la multiplication de $\wedge_{p}^{0}$. 
On vérifie facilement que (4.4.7) est un $S$-épimorphisme. Soit pour tout $n \leq v \leq p$,

$$
\mathfrak{U}[v]=\prod_{k=1}^{[p / v]+2} \mathfrak{U}(k)\left(\text { on pose } \mathfrak{U}(k)=\mathfrak{U}_{(0)}\right) .
$$

Soit, pour tout $n \leq v \leq p$,

$$
\gamma[v]: \mathfrak{U}[v] \longrightarrow \wedge_{n p}^{0}[v]
$$

le morphisme obtenu en composant

$$
\prod^{[p / v]+2} \operatorname{tr}_{\theta_{v}}{ }^{u} \varphi^{0}: \mathfrak{U}[v] \longrightarrow \prod^{[p / v]+2} \wedge_{n p}^{0}
$$

(c.à.d. le morphisme produit $([p / v]+2)$-fois de $\operatorname{tr}_{\theta_{v}}{ }^{u} \varphi^{0}: \mathfrak{H}_{(0)} \longrightarrow \wedge_{n p}^{0}$ $(p \geq v \geq n))$ avec le morphisme induit par le produit $\wedge_{n p}^{0}$. Il résulte de $(0.33)$ que (4.4.9) est fidèlement plat, et à fortiori que le morphisme

$$
\prod_{n \leq v \leq p} \gamma[v]: \prod_{n \leq v \leq p} \mathfrak{U}[v] \longrightarrow \prod_{n \leq v \leq p} \wedge_{n p}^{0}[v]
$$

l'est aussi.

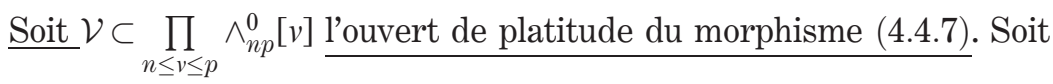

$$
W \subset \prod_{n \leq v \leq p} \mathfrak{U}[v]
$$

l'image inverse de $\mathcal{V}$ par $\prod_{n \leq v \leq p} \gamma[v]$. Soit $\mathcal{V}^{\prime} \subset \wedge_{n p}^{0}$ l'ouvert image de $\mathcal{V}$ par (4.4.7). Il résulte du théorème de platitude générique (cf. [10], pg. 153) et du théorème de platitude par fibres (compte tenu que (4.4.7) est un morphisme surjectif) que les fibres de $\mathcal{V}^{\prime}$ sont non-vides. On a alors que le morphisme

$$
\mathcal{V}^{\prime} \times \mathcal{V}^{\prime} \longrightarrow \wedge_{n p}^{0}
$$

induit par le produit est fidèlement plat (encore par le théorème de platitude par fibres). Soit $W \longrightarrow \wedge_{n p}^{0}$ le morphisme composé de la restriction de $\prod_{n \leq v \leq p} \gamma[v]$ à $W$ avec (4.4.7). Il résulte de ce qui précède que le morphisme

$$
W \times W \longrightarrow \wedge_{n p}^{0},
$$

obtenu à partir de la multiplication de $\wedge_{n p}^{0}$; est fidèlement plat. Pour tout $n \leq v \leq p$ on a un morphisme

$$
g[v]: \mathfrak{U}[v] \longrightarrow G
$$


composé de $\prod^{[p / v]+2} \operatorname{tr}_{\theta_{v}}{ }^{u} \alpha$ avec le morphisme $\prod^{[p / v]+2} G \longrightarrow G$ donné par la loi de composition de $G$. Soit

$$
\prod_{n \leq v \leq p} \mathfrak{U}[v] \longrightarrow G
$$

le morphisme obtenu à partir de $\prod_{n \leq v \leq p} g[v]$ et la loi de composition de $G$. Soit

$$
W \longrightarrow G
$$

le morphisme donné par la restriction de (4.4.13) à $W$. Soit

$$
W \times W \longrightarrow G
$$

le morphisme induit par la loi de composition de $G$ à partir de (4.4.14).

\section{Preuve. de la Proposition (4.4.1)}

Posons $\left(\wedge^{0}\right)^{(\mu)}=\wedge^{0} \times_{S} S_{\mu}$, où $S_{\mu}=\operatorname{Spec}\left(V / m^{\mu+1}\right) \ldots$ etc.

Soit ${ }^{u} f^{(\mu)}:\left(\wedge^{0}\right)^{(\mu)} \longrightarrow G^{(\mu)}$ la restriction de ${ }^{u} f$. On montre par un argument de présentation finie qu'il existe un entier $p \geq n$ et un $S_{\mu}$-homomorphisme ${ }^{u} f_{p}^{(\mu)}:\left(\wedge_{p}^{0}\right)^{(\mu)} \longrightarrow G^{(\mu)}$ qui permet de factoriser ${ }^{u} f^{(\mu)}$ à travers $\left(\wedge^{0}\right)^{(\mu)} \longrightarrow\left(\wedge_{p}^{0}\right)^{(\mu)}$. Il suffit alors de prouver que $\left(\wedge_{n p}^{0}\right)^{(\mu)} \subset \operatorname{Ker}^{u} f_{p}^{(\mu)}$. On a un diagramme commutatif

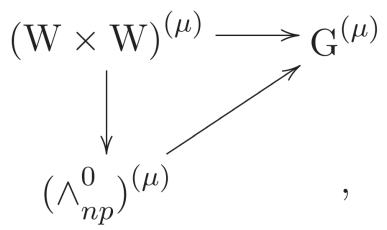

où la flèche horizontale est induite par (4.4.15), la verticale par (4.4.11), et la diagonale par la restriction de ${ }^{u} f_{p}^{(\mu)}$ à $\left(\wedge_{n p}^{0}\right)^{(\mu)} \subset\left(\wedge_{p}^{0}\right)^{(\mu)}$. Vu que la flèche verticale est fidèlement plate et la remarque (4.4.5), on en déduit que la restriction de ${ }^{u} f_{p}^{(\mu)}$ à $\left(\wedge_{n p}^{0}\right)^{(\mu)}$ est l'homomorphisme trivial. C.Q.F.D.

Soient $W_{(\infty)} \subset U_{(\infty)}$ le voisinage de la fibre spéciale $U_{(\infty) k}$ et

$$
\beta: W_{(\infty)} \longrightarrow G
$$

le $S$-morphisme donnés par (4.3.8). La fibre formelle $\widehat{\beta}$ de $\beta$ induit alors ${ }^{u} \bar{\alpha}$.

Le morphisme

$$
\overbrace{U \times \ldots \times U}^{(n+1)-\text { fois }} \longrightarrow \mathfrak{J}_{n}^{0}
$$


obtenu à partir de $\prod^{n+1} \varphi_{\mathfrak{C}}^{0}$ et le produit de $\mathfrak{J}_{n}^{0}$ est fini et fidèlement plat. Soit $W_{(\infty)} \subset U_{(\infty)}$ défini comme dans (4.3.8). Soit

$$
\prod^{n+1}\left(W_{(\infty)} \cap U\right) \longrightarrow \mathfrak{J}_{n}^{0}
$$

le morphisme donné par la restriction de (4.4.17) à l'ouvert $\prod^{n+1} W_{(\infty)} \cap U$. Son image est un ouvert de $\mathfrak{I}_{n}^{0}$ voisinage de la fibre spéciale $\left(\mathfrak{I}_{n}^{0}\right)_{k}$. (Ceci résulte du fait que $W_{(\infty)} \cap U$ est un voisinage de la fibre spéciale $U_{k}$ ) Soit

$$
\mathfrak{B}=\prod^{n+1}\left(W_{(\infty)} \cap U\right) \longrightarrow G
$$

le morphisme obtenu par composition de $\prod^{n+1} \beta$ (cf. (4.4.16)) et celui donné par la loi de composition de $G$

$$
\prod^{n+1} G \longrightarrow G .
$$

Soit $x$ un point de la fibre spéciale $\left(\mathfrak{J}_{n}^{0}\right)_{k}$. Notons par $\left(\mathfrak{J}_{n}^{0}\right)_{x}$ le $S$-schéma localisé en $x$ du $S$-schéma $\mathfrak{I}_{n}^{0}$, et par $\left(\prod^{n+1} U\right)_{x}$ (resp. $\left.\mathfrak{W}_{x}\right)$ l'image inverse de $\left(\mathfrak{J}_{n}^{0}\right)_{x} \operatorname{par}(4.4 .18)$ (resp. (4.4.19)). Il est clair que $\left(\prod^{n+1} U\right)_{x}$ (resp. $\mathfrak{B}_{x}$ ) est un $S$-schéma semi-local, et que $\left(\prod^{n+1} U\right)=\mathfrak{W}_{x}$.

LEMme 4.4.20. Le S-morphisme

$$
\left(\prod^{n+1} U\right)_{x} \longrightarrow G
$$

se "descend" en un S-morphisme

$$
\left(\mathfrak{J}_{n}^{0}\right)_{x} \longrightarrow G
$$

Le (4.4.20) résulte du

Lemme 4.21. Soit $X$ un S-schéma local noethérien. Soit $Y \longrightarrow X$ un $S$ morphisme fidèlement plat et fini. Soit $Z$ un $S$-schéma de présentation finie et séparé

Notons par $\widehat{X}$ (resp. $\widehat{Y}, \widehat{Z})$ le complété formel de $X$ (resp. $Y, Z)$ le long de la fibre spéciale $X_{k}\left(\right.$ resp. $Y_{k}, Z_{k}$ ). Soit $f: \widehat{X} \longrightarrow \widehat{Z}$ un $S$-morphisme formel tel qu'il existe un $S$-morphisme $Y \longrightarrow Z$ rendant commutatif le dia- 
gramme suivant

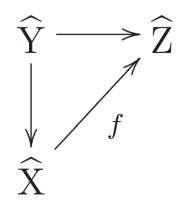

Alors le S-morphisme $Y \longrightarrow Z$ se descend en un $S$-morphisme $g: X \longrightarrow Z$ tel que $\widehat{g}=f$.

La preuve de (4.4.21) résulte par descente fidèlement plate à partir des isomorphismes

$$
\operatorname{omb}(\widehat{Y}) \simeq \operatorname{omb}(\widehat{X}) \times Y
$$

et

$$
\operatorname{omb}(Y \times Y)^{\wedge} \simeq \operatorname{omb}(\widehat{X}) \times(Y \times Y),
$$

qui proviennent du fait que $Y \longrightarrow X$ est fini.

Le lemme (4.4.20) résute de (4.4.21) en posant $Y=\left(\prod^{n+1} U\right)_{x}$, $X=\left(\mathfrak{J}_{n}^{0}\right)_{x}$, et $Z=G$. On prend comme $f$ le morphisme induit par ${ }^{u} f_{n}$ (cf. (4.4.1)) et comme le morphisme $Y \longrightarrow Z$ celui induit par (4.4.19). Il résulte de (4.4.20) l'existence d'un ouvert $W^{\prime} \subset \mathfrak{J}_{n}^{0}$ voisinage de la fibre spéciale $\left(\mathfrak{J}_{n}^{0}\right)_{k}$, et d'un $S$-morphisme

$$
W^{\prime} \longrightarrow G
$$

tel que par passage au complété formel $\widehat{W}^{\prime}=\left(\mathfrak{I}_{n}^{0}\right)^{\wedge}$ coïncide avec le $S$-homomorphisme

$$
\widehat{\mathfrak{J}}_{n}^{0} \longrightarrow \widehat{\wedge}_{n}^{0} \stackrel{{ }^{u} f_{n}}{\longrightarrow} \widehat{G}
$$

D'autre part le diagramme suivant est commutatif

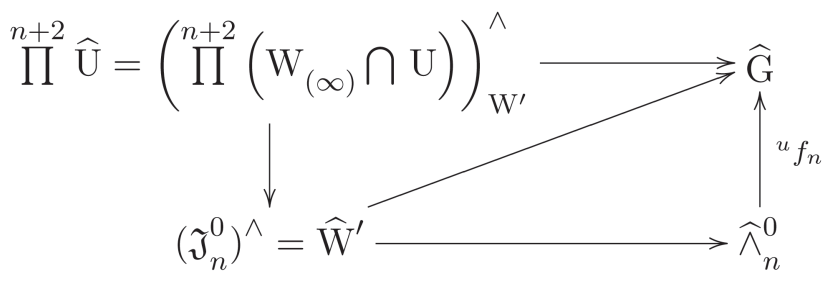

Le morphisme horizontal (resp. vertical) étant donné par $\prod^{n+2} \widehat{\beta}=\prod^{n+2} u \bar{\alpha}$ (resp. $\prod^{n+2} \varphi_{\mathfrak{C}}^{0}$ ) suivi de $\prod^{n+2} \widehat{G} \longrightarrow \widehat{G}$ (resp. $\prod^{n+2} \widehat{\mathfrak{J}}_{n}^{0} \longrightarrow \widehat{\mathfrak{J}}_{n}^{0}$ ) induit par la loi de 
composition de $\widehat{G}$ (resp. $\widehat{\Im}_{n}^{0}$ ). La commutativité résulte de ${ }^{u} \bar{\alpha}={ }^{u} f_{n} \circ \widehat{\varphi}_{\mathbb{\mathbb { C }}}^{0}$. Comme le point générique $\xi$ de $\prod^{n+2} W_{(\infty)} \cap U$ appartient à l'image du morphisme

$$
\operatorname{omb}\left(\prod^{n+2}\left(W_{(\infty)} \cap U\right)_{W^{\prime}}^{\wedge}\right) \longrightarrow \prod^{n+2} W_{(\infty)} \cap U,
$$

on démontre que le diagramme suivant

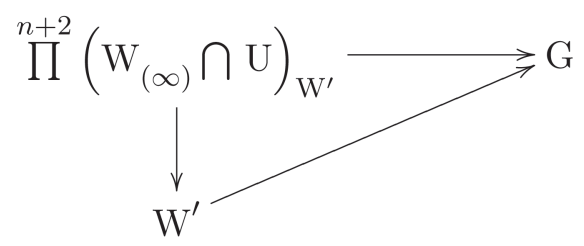

est commutatif. (Noter que le morphisme horizontal est la restriction à $n+2$

$\prod\left(W_{(\infty)} \cap U\right)_{W^{\prime}}$ de (4.4.19) et que le diagramme (4.4.22) s'obtient par passage à la fibre formelle de (4.4.23)). Comme le morphisme horizontal de (4.4.23) admet un prolongement à $\prod^{n+2}\left(W_{(\infty)} \cap U\right)$, compte tenu que $\prod^{n+2}\left(W_{(\infty)} \cap U\right) \longrightarrow \mathfrak{I}_{n}^{0}$ est fidèlement plat (On a d'après (4.3.8) que $\mathfrak{u}_{(0)} \subset W_{(\infty)} \cap U$. Il suffit alors d'appliquer (0.33)), on a alors

Lemme 4.4.24. Le S-morphisme $W^{\prime} \longrightarrow G$ admet un prolongement $\mathfrak{I}_{n}^{0} \longrightarrow G$ qui est en fait un $S$-homomorphisme. On a aussi un diagramme commutatif

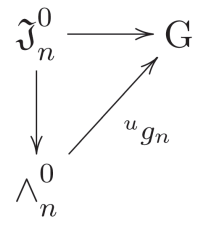

de S-homomorphismes tel que ${ }^{u} \alpha={ }^{u} g_{n} \circ{ }^{u} \varphi_{T}^{0}$.

Conclusion (4.4.25). D'après (4.1.6) on a $\alpha={ }^{m} \alpha^{u} \alpha$, avec ${ }^{m} \alpha={ }^{m} g \circ{ }^{m} \varphi_{T}^{0}$ et ${ }^{u} \alpha={ }^{u} g_{n} \circ \varphi_{T}^{0}$ (cf. (4.3.13) et (4.4.24)). Soit $g={ }^{m} g^{u} g_{n}$ : $\mathfrak{I}_{n}^{0} \longrightarrow G$ le $S$-homomorphisme défini à partir de la décomposition $\mathfrak{J}_{n}^{0}=\mathrm{G}_{m_{S}} \times \wedge_{n}^{0}$. Compte tenu que $\varphi_{T}^{0}=\left({ }^{m} \varphi_{T}^{0},{ }^{u} \varphi_{T}^{0}\right)$ on a $\alpha=g \circ \varphi_{T}^{0}$. L'unicité de la factorisation formelle (cf. (3.17)) $\widehat{\alpha}=f \circ \widehat{\varphi}_{T}^{0}$ entraîne que $\widehat{g}=f$. 
On achève ainsi la démonstration du théorème (1.4.4) dans le cas d'un trait complet de base $S$. En vertu de (1.4.6) ceci entraîne le théorème (1.4.4) pour un trait de base.

\section{Preuve du théorème (1.4.4)}

Montrons d'abord la

Proposition 5.1. Soit $A$ un anneau noethérien local réduit. Notons par $\widehat{A}$ le complété formel de A pour la topologie $m_{A}$-adique. (On pose $\mathfrak{U}=\operatorname{Spec}(A[[T]]))$. Soit $\alpha \in G(\mathfrak{H})$ tel qu'il existe un $\widehat{A}$-homomorphisme formel $f: \widehat{\mathfrak{J}}^{0} \longrightarrow \widehat{G}$ avec $\widehat{\alpha}=f \circ \widehat{\varphi}_{T}^{0}$. Il existe alors $g \in \operatorname{Hom}_{A-g r}\left(\mathfrak{J}^{0}, G\right)$ tel que $\alpha=g \circ \varphi_{T}^{0}$.

Preuve. Supposons d'abord que A est intègre. Soit $V$ un anneau de valuation discrète qui majore $A$. Soit $K$ le corps des fractions de A. Noter que $K$ est aussi le corps des fractions de V. Soit $\mathfrak{U}^{\prime}=\operatorname{Spec}\left(V[[T]]\left[T^{-1}\right]\right)$. Soit $\alpha^{\prime}$ le morphisme composé suivant

$$
\mathfrak{\mathfrak { l } ^ { \prime }} \longrightarrow \mathfrak{U} \stackrel{\alpha}{\longrightarrow} G .
$$

D'après $\S 4$ il existe un $V$-homomorphisme $g^{\prime}: \mathfrak{I}_{V}^{0} \longrightarrow G_{V}$ tel que $\alpha^{\prime} \equiv g^{\prime} \circ \varphi_{T}^{0}(\bmod G(V[[T]]))$. L'hypothèse donne $\widehat{\alpha}^{\prime}=\widehat{g}^{\prime} \circ \widehat{\varphi}_{T}^{0}$, d'où il résulte que $\alpha^{\prime}=g^{\prime} \circ \varphi_{T}^{0}$. On vérifie ensuite que la fibre générique $g_{K}^{\prime}$ de $g^{\prime}$ est un K-homomorphisme de $\mathfrak{J}_{K}^{0}$ dans $G_{K}$, tel que $\alpha_{K}=g_{K}^{\prime} \circ\left(\varphi_{T}^{0}\right)_{K}$. On peut même supposer par un argument de locale présentation finie qu'il existe un K-homomorphisme pour $n$ convenable

$$
g_{K}:\left(\mathfrak{J}_{n}^{0}\right)_{K} \longrightarrow G_{K}
$$

tel que $\alpha_{K}=g_{K} \circ\left(\varphi_{T}^{0}\right)_{K}$.

Montrons que $g_{K}$ admet un prolongement $\grave{a} \mathfrak{J}_{n}^{0}$. On a le diagramme commutatif suivant

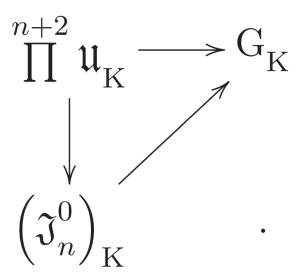


La flèche verticale admet un prolongement $\stackrel{n+2}{\prod} \mathfrak{U} \longrightarrow G$, à savoir, le composé de $\prod^{n+2}$ a avec $\prod^{n+2} G \longrightarrow G$. D'où $g_{K}$ admet aussi un prolongement $g \grave{a}$ $\mathfrak{J}_{n}^{0}$, qui doit être nécessairement un A-homomorphisme.

Supposons maintenant A réduit. Soit I l'ensemble des composantes irréductibles de Spec (A). Pour tout $s \in I$ notons par $K_{s}$ le corps des fractions de la composante irréductible s. Posons $S^{\prime}=S p e c\left(\prod_{s \in I} K_{s}\right)$. D'après ce qui précède (en raisonnant composante par composante) on peut trouver un $S^{\prime}$-homomorphisme $g_{S^{\prime}}: \mathfrak{J}_{n}^{0} \longrightarrow G_{S^{\prime}}$ tel que $\alpha_{S^{\prime}}=g_{S^{\prime}} \circ\left(\varphi_{T}^{0}\right)_{S^{\prime}}$. On conclut à l'existence d'un S-homomorphisme g prolongeant $g_{S^{\prime}}$ par un argument de fidèle platitude calqué sur celui qui précède.

C.Q.F.D.

Soit $A$ un anneau noethérien. Montrons (1.4.4) si $S=\operatorname{Spec}(A)$. La proposition (3.1) permet de supposer que $A$ est réduit. Soit $s$ un point de $S$, et notons par $S_{s}$ le localisé de $S$ en $s$. Soit $\alpha \in G(\mathfrak{H})$. Par (5.1) il existe un $S_{s}$-homomorphisme $f_{s}: \mathfrak{J}_{S_{s}}^{0} \longrightarrow G_{S_{s}}$ tel que $\alpha_{\left\{S_{s}\right\}} \equiv$ $f_{s} \circ\left(\varphi_{T}^{0}\right)_{S_{s}}\left(\bmod G\left(\operatorname{omb}\left(\mathfrak{X}_{S_{s}}\right)\right)\right)$. Donc $\alpha_{S_{s}} \equiv f_{s} \circ\left(\varphi_{T}^{0}\right)_{S_{s}}$. On peut alors trouver un ouvert $U_{s}$ voisinage de $s$, et un $U_{s}$-homomorphisme $f_{U_{s}}: \mathfrak{J}_{U_{s}}^{0} \longrightarrow G_{U_{s}}$ tel que $\alpha_{U_{s}} \equiv f_{U_{s}} \circ\left(\varphi_{T}^{0}\right)_{U_{s}}\left(\bmod G\left(\operatorname{omb}\left(\mathfrak{x}_{U_{s}}\right)\right)\right)$. On conclut par (1.4.6) qu'il existe un $S$-homomorphisme $f: \mathfrak{J}^{0} \longrightarrow G$ tel que $\alpha \equiv f \circ \varphi_{T}^{0}(\bmod G(\operatorname{omb}(\mathfrak{X})))$. Ce qui achève la preuve de (1.4.4).

\section{Autodualité de $\Im$}

Soit $A$ un anneau noethérien. Soit $S=\operatorname{Spec}(A)$. Il résulte alors de (1.6.6) en posant $G=\underline{\mathrm{G}}_{m S}$ un isomorphisme

$$
\operatorname{Hom}_{S-g r}\left(\Im, \underline{\mathrm{G}}_{m S}\right) \simeq \mathrm{G}_{m S}(\mathfrak{l} \mathfrak{l})=\Im(S) .
$$

On a alors l'isomorphisme suivant de foncteurs en groupes sur la catégorie des $S$-schémas noethériens affines

$$
\underline{\operatorname{Hom}}_{S-g r}\left(\Im, \underline{\mathrm{G}}_{m S}\right) \simeq \Im .
$$

En fait (6.1) est la restriction d'un isomorphisme entre les foncteurs correpondants sur la catégorie des schémas affines, qui fait de $\Im$ sont propre dual de Cartier. Supposons d'abord que $A$ est une algèbre sur le corps des nombres rationnels Q. Pour tout entier $m \in Z$ et toute section $w$ de $\Im$ posons

$$
\partial_{m} w=\operatorname{Res}\left(T^{m} d w / w\right) .
$$


On définit un $S$-morphisme

$$
\langle\ldots, \ldots\rangle: \Im \times \Im \longrightarrow \underline{\mathrm{G}}_{m} \mathrm{~s}
$$

comme suit. Soient

$$
\begin{aligned}
u=T^{\varepsilon(u)}\left(1+a_{-1} t^{-1}+\ldots+a_{-v} t^{-v}\right)\left(a_{0}+a_{1} t+\ldots\right)= \\
t^{\varepsilon(u)} u^{\prime} a_{0} u^{\prime \prime} \in \underline{G}_{m S}\left(A[[t]]\left[t^{-1}\right]\right)
\end{aligned}
$$

et

$$
\begin{aligned}
& v=T^{\varepsilon(v)}\left(1+\alpha_{-1} t^{-1}+\ldots+\alpha_{-n} t^{-n}\right)\left(\alpha_{0}+\alpha_{1} t+\ldots\right)= \\
& t^{\varepsilon(v)} v^{\prime} \alpha_{0} v^{\prime \prime} \in \underline{\mathbb{G}}_{m S}\left(A[[t]]\left[t^{-1}\right]\right) .
\end{aligned}
$$

On pose :

$$
\langle u, v\rangle=(-1)^{\varepsilon(u) \varepsilon(v)}\left(\frac{\alpha_{0}^{\varepsilon(u)}}{a_{0}^{\varepsilon(v)}}\right) \frac{\exp \left(\sum_{m>0} \partial_{m} u \partial_{-m} v / m\right)}{\exp \left(\sum_{m>0} \partial_{m} v \partial_{-m} u / m\right)}
$$

Notons que la somme $\sum_{m>0} \partial_{m} u \partial_{-m} v / m$ (resp. $\left.\sum \partial_{m} v \partial v_{-m} u / m\right)$ s'étend à un nombre fini de termes non nuls et définit alors un élément nilpotent de $A$.

Il est clair que $\langle\ldots, \ldots\rangle$ est un $S$-bi-homomorphisme qui vérifie la condition d'anti-symétrie

$$
\langle u, v\rangle=\langle v, u\rangle^{-1} \quad\left(\operatorname{resp} .\langle u, u\rangle=(-1)^{\varepsilon(u)}\right) .
$$

Vérifions qu'étant donné la section $v$ de $\underline{\mathbb{G}}_{m S}$ au-dessus de $\mathfrak{U}$ alors le $S$ homomorphisme $f: \Im \longrightarrow \underline{\mathbb{G}}_{m S}$ qui lui correspond d'après (1.6.6) est donné par

$$
f(u)=\langle u, v\rangle
$$

Noter que l'on a l'identité suivante

$$
d\left(1-t T^{-1}\right) /\left(1-t T^{-1}\right)=\left(\sum_{m>0} t^{m} T^{-m-1}\right) d T
$$

qui entraîne pour tout entier $m>0$

$$
\operatorname{Res}\left(T^{m} d\left(1-t T^{-1}\right) /\left(1-t T^{-1}\right)=t^{m} .\right.
$$

D'autre part l'identité (2.15) dans $\left(A[[t]]\left[t^{-1}\right]\right)[[T]]\left[T^{-1}\right] d T$ donne pour tout 
entier $m>0$

$$
\operatorname{Res}\left(T^{-m} d\left(1-t^{-1} T\right)^{-1} /\left(1-t^{-1} T\right)^{-1}\right)=t^{-m}
$$

Notons que pour tout entier $m>0$ on a les identités suivantes

(6.10) $\partial_{m} u=\partial_{m} u^{\prime}$ et $\partial_{m} v=\partial_{m} v^{\prime}$ (resp. $\partial_{-m} u=\partial_{-m} u^{\prime \prime}$ et $\partial_{-m} v=\partial_{-m} v^{\prime \prime}$ ).

Il n'y a qu'un nombre fini d'entiers $m>0$ tel que $\partial_{m} u \neq 0$ (resp. $\partial_{m} v \neq 0$ ), et d'autre part $\partial_{m} u$ (resp. $\partial_{m} v$ ) est un élément nilpotent de $A$ pour tout $m$. On a aussi

$$
v^{\prime \prime}=\exp \left(\sum_{m>0} \frac{\partial_{-m} v t^{m}}{m}\right)\left(\operatorname{resp} \cdot v^{\prime}=\exp \left(\sum_{m>0} \frac{\partial_{m} v t^{-m}}{m}\right)\right) .
$$

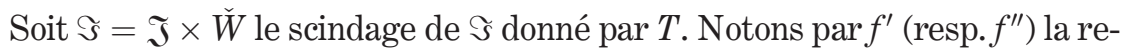
striction de $f$ à $\mathfrak{J}$ (resp. $\breve{W})$. Soit $f_{\text {omb }}^{\prime \prime}: \operatorname{omb}(\breve{W}) \longrightarrow \underline{G}_{m S}$ le $S$-morphisme induit par $f^{\prime \prime}$ d'après (1.5.5). Pour vérifier que le $S$-homomorphisme $f$ donne lieu, selon la construction de (1.6), à la section $v=T^{\varepsilon(v)} v^{\prime} \alpha_{0} v^{\prime \prime}$ il suffit de voir que :

(i) - Le $S$-homomorphisme $f^{\prime}$ composé avec la section $-T(t-T)^{-1}$ de $\mathfrak{J}$ au-dessus de $\mathfrak{U}$ donne $\alpha_{0} v^{\prime}$;

(ii) - le composé de la section $\left(1-t T^{-1}\right)$ de omb( $\left.\breve{W}\right)$ au-dessus de omb( $\left.\mathfrak{X}\right)$ avec $f_{\text {omb }}^{\prime \prime}$ donne $v^{\prime \prime}$.

En effet, noter que le morphisme

$$
f_{\mathrm{omb}}: \Im_{\mathrm{omb}} \simeq \mathfrak{J} \times \mathrm{omb}(\check{W}) \longrightarrow \underline{\mathrm{G}}_{m S}(\mathrm{cf} .(1.6 .3))
$$

est obtenu comme le composé de $\left(f^{\prime}, f_{\text {omb }}^{\prime \prime}\right)$ avec le morphisme $\underline{\mathrm{G}}_{m S} \times \underline{\mathrm{G}}_{m S} \longrightarrow \underline{\mathrm{G}}_{m S}$ donné par la loi de composition $\underline{\mathrm{G}}_{m S}$. Il en résulte, compte tenu de la description du morphisme d'Abel-Jacobi donnée à la suite de la définition (1.5.18), que (i) et (ii) entraînent que $v$ correspond à $f$ selon la construction de (1.6), vu l'unicité du morphisme $f$ qui factorise $v$.

Soit $u=-T(t-T)^{-1}$ on a alors (Noter que $\varepsilon(u)=1$ )

$$
\begin{aligned}
& \text {.12) }\langle u, v\rangle=\left\langle-T(t-T)^{-1}, v\right\rangle= \\
& =(-1)^{\varepsilon(v) \varepsilon(u)}\left(\alpha_{0}^{\varepsilon(u)} /\left(-t^{-1}\right)^{\varepsilon(v)}\right) \exp \left(\sum_{m>0} \frac{\partial_{m} v \partial_{m}\left(1-t^{-1} T\right)^{-1}}{m}\right)^{-1}=\alpha_{0} v^{\prime},
\end{aligned}
$$

comme il résulte de (6.9) et de (6.11), ce qui montre (i). D'autre part on a

$$
\left\langle 1-t T^{-1}, v\right\rangle=\exp \left(\sum_{m>0} \frac{\partial_{-m} v \partial_{m}\left(1-t T^{-1}\right)}{m}\right)=v^{\prime \prime},
$$

ce qui montre (ii), et finalement que $v$ provient de $f$. 
Détaillons maintenant comment on passe de l'accouplement (6.4) de $\Im$ défini sur le corps des rationnels $\mathrm{Q}$ à un accouplement défini sur Z $\mathrm{Z}$.

Soit $\mathrm{A}^{\mathbb{N}^{*}}$ le foncteur en anneaux dont les sections au-dessus d'un anneau $A$ est l'ensemble des suites $\left(a_{n}\right)(n \geq 1)$ de $A$ muni de la loi d'addition (resp. de multiplication) produit. Soit $\mathrm{A}^{\left(-N^{*}\right)}$ le foncteur en anneaux dont les sections au-dessus d'un anneau $A$ est l'ensemble des suites $\left(a_{-n}\right)(n \geq 1)$ de $A$, tel qu'il existe un entier $N$ vérifiant : pour tout $n \geq 1$ on a $a_{-n}^{N}=0$, et $a_{-(N+1)}=a_{-(N+2)}=\ldots=0$, muni de la loi d'addition (resp. multiplication) produit. Soit W (resp. W) le foncteur en anneaux défini par le foncteur en ensembles sous-jacent à $\mathbb{A}^{\mathbb{N}^{*}}$ (resp. $\left.\mathbb{A}^{\left(-\mathbb{N}^{*}\right)}\right)$, muni de l'unique loi d'addition + et l'unique loi de multiplication $*$ qui rend le morphisme fonctoriel

$$
w_{\infty}: \mathbb{W} \longrightarrow \mathrm{A}^{\mathbb{N}^{*}}\left(\text { resp. } w_{-\infty}: \check{W} \longrightarrow \mathrm{A}^{\left(-\mathbb{N}^{*}\right)}\right),
$$

défini par

$$
w_{\infty}\left(\left(Y_{n}\right)\right)=\left(\sum_{n d=m} n Y_{n}^{d}\right)\left(\operatorname{resp} . w_{-\infty}\left(\left(X_{-n}\right)\right)=\left(\sum_{n d=m} n X_{-n}^{d}\right)\right)
$$

un homomorphisme de foncteurs en anneaux.

On a un isomorphisme de groupes

$$
E_{\infty}: \mathbb{W} \longrightarrow \wedge^{0} \quad\left(\text { resp. } E_{-\infty}: \check{W} \longrightarrow \check{W}\right)
$$

défini par

$$
E_{\infty}\left(\left(Y_{n}\right)\right)=\prod_{n=1}^{\infty}\left(1-Y_{n} T^{n}\right)^{-1}\left(\text { resp. } E_{-\infty}\left(\left(X_{-n}\right)\right)=\prod_{n=1}^{\infty}\left(1-X_{-n} T^{-n}\right)\right)
$$

Notons par $W_{Q}, W_{Q}, A_{Q}^{N^{*}}$,...etc. respectivement la fibre du foncteur $W, W$ W, $\mathrm{A}^{N^{*}}$,...etc. au-dessus de Q. On peut alors caractériser $E_{\infty}$ (resp. $E_{-\infty}$ ) comme étant le seul homomorphisme qui rend commutatif le diagramme suivant
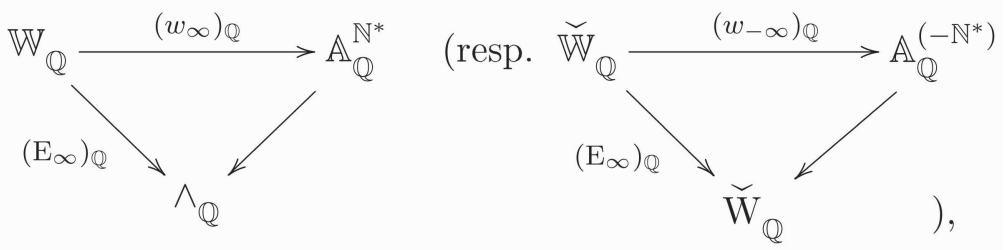

où la flèche oblique droite fait correspondre à la section $\left(a_{n}\right)\left(\right.$ resp. $\left.\left(a_{-n}\right)\right)$ de 
$\mathbb{A}^{N^{*}}\left(\right.$ resp. $\left.\mathrm{A}^{\left(-N^{*}\right)}\right)$ la section $\exp \left(\sum_{m>0} \frac{a_{m} t^{m}}{m}\right)\left(\operatorname{resp} \exp \left(-\sum_{m>0} \frac{a_{-m} t^{-m}}{m}\right)\right)$. La pré-image de $v \in \widetilde{W}_{\mathrm{Q}}$ (resp. $\wedge_{\mathrm{Q}}$ ) par cette flèche est donnée par la section $\left(v_{-m}\right)$ de $\mathrm{A}_{\mathrm{Q}}^{\left(-N^{*}\right)}$ où $v_{-m}=\partial_{m} v(m>0)$ (resp. la section $\left(v_{m}\right)$ de $\mathrm{A}_{\mathrm{Q}}^{\mathbb{N}^{*}}$ où $\left.v_{m}=\partial_{-m} v(m>0)\right)$ (cf. (6.2)).

On a un morphisme

$$
\mathrm{A}^{\mathrm{N}^{*}} \times \mathrm{A}^{\left(-\mathrm{N}^{*}\right)} \longrightarrow \mathrm{A}^{\left(-\mathrm{N}^{*}\right)}
$$

défini par $\left(y_{n}\right)\left(x_{-n}\right)=\left(y_{n} x_{-n}\right)$ qui fait de $\mathrm{A}^{\left(-\mathbb{N}^{*}\right)}$ un $\mathrm{A}^{\mathbb{N}^{*}}$-module. Soit

$$
*^{\prime}: W \times \check{W} \longrightarrow \check{W}
$$

le morphisme défini à partir de (6.19), de $w_{\infty}$ et de $w_{-\infty}$ qui fait de $\mathscr{W}_{\text {un }}$ Wmodule. Soit

$$
\check{W} \longrightarrow \underline{\mathbb{G}}_{m S}
$$

le $S$-homomorphisme faisant correspondre $1+\sum_{i} \alpha_{-i}$ à la section
$1+\alpha_{-1} t^{-1}+\ldots+\alpha_{-n} t^{-n}$. Notons par

$$
\bar{E}: \check{W} \longrightarrow \underline{G}_{m S}
$$

le homomorphisme composé de $E_{-\infty}$ avec (6.21).

Soient $v=E_{-\infty}(\underline{x}) T^{\varepsilon(v)} \alpha E_{\infty}(y)$ et $u=E_{-\infty}\left(\underline{x}^{\prime}\right) T^{\varepsilon(u)} a E_{\infty}\left(y^{\prime}\right)$, avec $\underline{x}, \underline{x}^{\prime} \in \breve{W}, a, \alpha \in \Gamma\left(S, \mathcal{O}_{S}^{*}\right), \underline{y}, \underline{y^{\prime}} \in \bar{W}$. On redéfinit (6.4), en général, en posant

$$
\langle u, v\rangle=(-1)^{\varepsilon(u) \varepsilon(v)}\left(\frac{\alpha^{\varepsilon(u)}}{a^{\varepsilon(v)}}\right)\left(\bar{E}\left(\underline{x}^{\prime} *^{\prime} \underline{y}\right)\right)^{-1} \bar{E}\left(\underline{x} *^{\prime} \underline{y^{\prime}}\right) .
$$

Il résulte par construction que l'accouplement (6.22) coïncide avec (6.4) sur la catégorie des Q-algèbres. Soit $v$ comme ci-dessus. Posons $\underline{y}=\left(y_{n}\right)$ et $\underline{x}=\left(x_{-n}\right)$. On vérifie immédiatement

$$
\begin{gathered}
\left(1-t T^{-1}\right) *^{\prime}\left(y_{1}, y_{2}, \ldots\right)=\left(t y_{1}, t^{2} y_{2}, \ldots\right) \\
\left(\operatorname{resp.}\left(1-t^{-1} T\right) *^{\prime}\left(x_{-1}, x_{-2}, \ldots\right)=\left(t^{-1} x_{-1}, t^{-2} x_{-2}, \ldots\right)\right) .
\end{gathered}
$$

On a alors

$$
\left\langle 1-t T^{-1}, v\right\rangle=E_{\infty}(\underline{y})\left(\operatorname{resp} .\left\langle 1-t^{-1} T, v\right\rangle=E_{-\infty}(\underline{x})\right),
$$

ce qui nous permet de montrer que l'accouplement $\langle\ldots, \ldots\rangle$ donne lieu à un homomorphisme $\langle\ldots, v\rangle: \Im \longrightarrow \underline{G}_{m S}$ qui correspond bien à la section $v$ de $\underline{\mathrm{G}}_{m S} \operatorname{par}(1.6 .6)$. 


\section{REFERENCES}

[1] P. CARTIER, Groupes formels associés aux anneaux de Witt généralisés, CRAS, t 265 (1967), série A-49.

[2] P. CARTIER, Modules associés Á un groupe formel commutatif, CRAS, t 265 (1967), série A-129.

[3] C. Contou-Carrère, La jacobienne généralisée d'une courbe relative ; construction et propriété universelle de factorisation, CRAS, t 289 (1979).

[4] C. Contou-CARRÈre, Corps de classes local géométrique relatif, CRAS, t. 292 (1981).

[5] C. Contou-Carrère, Jacobiennes globales relatives, in: The Grothendieck Festschrift, Vol. II, Progr. Math. 87, Birkhauser, Boston 1990, P. Cartier, et al., eds., pp. 69-109.

[6] M. Demazure - P. Gabriel, Groupes algébriques , North Holland, 1971.

[7] A. Grothendieck (M. Artin - P. Deligne - ... etc), Théorie des topos et cohomologie étale des schémas, SGA IV, vol.3 , Exp. XVIII, Springer-Verlag, L. N. $n^{\circ} 305$.

[7 bis] A. Grothendieck - M. Demazure, Schémas en groupes, SGA III, Vol. 1, Springer-Verlag, L.N. $n^{\circ} 151$.

[8] A. Grothendieck - J. Dieudonné, Elements de géométrie algébrique, Springer-Verlag 1971.

[9] A. Grothendieck - J. Dieudonné, EGA III (première partie), Etude cohomologique des faisceaux cohérents, Publications Mathématiques de l'IHES, vol. $n^{0} 11$.

[10] A. Grothendieck - J. Dieudonné, E.G.A. IV (deuxième partie), Etude locale des schémas et des morphismes de schémas, Publications Mathématiques de l'IHES, vol. $n^{0} 24$.

[11] A. Grothendieck - J. Dieudonné, EGA IV (quatrième partie), Etude locale des schémas et des morphismes de schémas, Publications Mathématiques de l'IHES, vol. $n^{0} 32$.

[12] J. Lipman, Rationnal singularities (Appendix), Volume dédié au professeur Oscar Zariski, Publications de l'IHES, vol. $n^{0}$ 36, pp. 273.

[13] D. Mumford, Lectures on curves on an algebraic surface, Princeton University Press 1966.

[14] J. P. SERRE, Groupes algébriques et corps de classes, Hermann 1959.

[15] P. Samuel, Séminaire Bourbaki, Exp. nº 145 (1956-57).

[16] R. Hartshorne, Residues and Duality, Springer-Verlag, L. N. n 20 (1966).

[17] Y. KaWAda - I. SATake, Class formations II, Journal of the Faculty of Sciences, University of Tokyo, vol VII, Part 3 (1956).

[18] A. WeIL, Variétés Abéliennes, Hermann 1948.

\section{Références supplementaires}

[A-R] G. ANDERSON - F. P. Romo, Simple proof of classical explicit reciprocity laws on curves using determinant groupoids over an artinian ring, Comm. in Alg., Vol. 32, $\mathrm{n}^{\mathrm{o}} 1$ (2004). 
[Bei] A. Beilinson, $\varepsilon$-factors for the period determinants of curves, in: Motives and Algebraic Cycles, A Celebration in Honour of Spencer Bloch (AMS) (2009).

[Bei-Dr] A. Beilinson - V. G. Drinfeld, Chiral algebras, AMS 2004.

[B-B-E] A. Beilinson - S. J. Bloch - H. Esnault, e-factors for Gauss-Manin determinants, Moscow mathematical journal (2002), number 3, pp. 432477.

[D] P. Deligne, Le symbole moderé, Publications Mathématiques de l'IHES, vol. 73, (1991).

[E-L-O] A. Efimov - V. A. Lunts - D. OrLov, Deformation theory of objects in homothopy and derived categories III: Abelian categories, arXiv: math/0702840v3 [mathAG], 04/12/2008.

[KR] K. Rulling, The generalised De Rham-Witt complex over a field is a complex of zero cycles, Journal of Algebraic Geometry, 16 (2007), $\mathrm{n}^{\mathrm{o}} 1$, pp. 109-169.

[K-V] M. Kapranov - E. VAsserot, Formal loops II: A local Riemann-Roch theorem for determinantal gerbs, AScENS, t. 40 (2007).

[Luo] Zhenbin Luo, Contou-Carrère symbol via iterated integrals and its reciprocity law, arXiv:1003-1431v1[math.DG].

[PA] P. AmBrus, On the kernel and the image of the rigid analytic regulator in positive characteristic, arXiv:0912.3115v1 [mathAG], 16/12/2009.

[A-N-P] A. N. PARSHIN, Representations of higher adelic groups and arithmetic, Proceedings of the International Congress of Mathematicians, Volume I, pp. 362-392, Hindustan Book Agency, New Dehli, 2010, ArXiv:1012.0486 [math.NT] .

[PR] P. Romo, A direct proof of the Steinberg property of the Contou-Carrère symbol, Journal of Algebra 319 (2008), pp. 3164-3174.

[O-Z] D. OsIPOV - X. ZHU, A categorical proof of the Parshin reciprocity laws on algebraic surfaces, Algebra and Number Theory, vol. 5 (2011), nº 3, 289 337 (ArXiv: 1012.4848 [math.AG]).

Manoscritto pervenuto in redazione 12 Ottobre 2011. 